\title{
A Damage Mechanics Based Approach to Structural Deterioration and Reliability
}

Manuscript Completed: April 1997

Date Published: February 1998

Prepared by

B. Bhattacharya, B. Ellingwood, JHU

Oak Ridge National Laboratory

Managed by Lockheed Martin Energy Research Corporation, Inc.

Oak Ridge National Laboratory

Oak Ridge, TN 37831-8056

Subcontractor:

The Johns Hopkins University

Department of Civil Engineering

Baltimore, MD 21218

W. E. Norris, NRC Project Manager

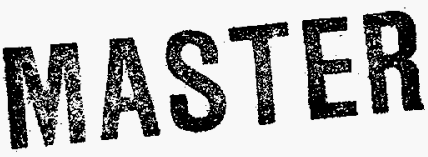

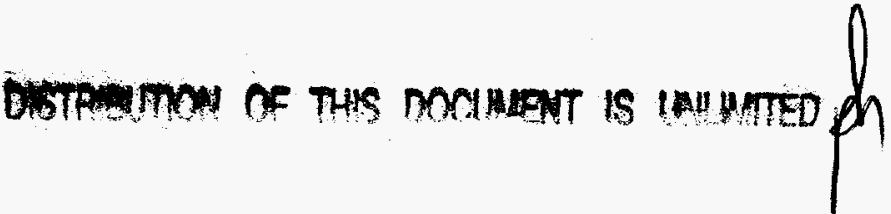

Prepared for

Division of Engineering Technology

Office of Nuclear Regulatory Research

U.S. Nuclear Regulatory Commission

Washington, DC 20555-0001

NRC Job Code J6043

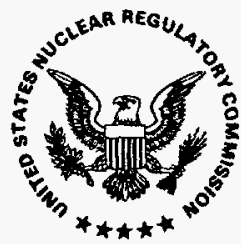




\section{DISCLAIMER}

This report was prepared as an account of work sponsored by an agency of the United States Government. Neither the United States Government nor any agency thereof, nor any of their employees, makes any warranty, express or implied, or assumes any legal liability or responsibility for the accuracy, completeness, or usefulness of any information, apparatus, product, or process disclosed, or represents that its use would not infringe privately owned rights. Reference herein to any specific commercial product, process, or service by trade name, trademark, manufacturer, or otherwise does not necessarily constitute or imply its endorsement, recommendation, or favoring by the United States Government or any agency thereof. The views and opinions of authors expressed herein do not necessarily state or reflect those of the United States Government or any agency thereof. 


\section{DISCLAIMER}

Portions of this document may be illegible electronic image products. Images are produced from the best available original document. 


\section{Abstract}

Structural deterioration often occurs without perceptible manifestation. However, the existing methods of modeling structural deterioration usually need measurable flaws to be applicable. Moreover, the existing methods of predicting service-life are mostly empirical in nature and are generally unable to provide estimates of residual strength of a degrading structure.

Continuum damage mechanics (CDM) defines structural damage in terms of the material microstructure, and relates the damage variable to the macroscopic strength or stiffness of the structure. This enables one to predict the state of damage prior to the initiation of a macroscopic flaw, and allows one to estimate residual strength/service life of an existing structure.

The accumulation of damage is a dissipative process that is governed by the laws of thermodynamics. Partial differential equations for damage growth in terms of the Helmholtz free energy are derived from fundamental thermodynamical conditions. Closedform solutions to the equations are obtained under uniaxial loading for ductile deformation damage as a function of plastic strain, for creep damage as a function of time and for fatigue damage as function of number of cycles. The proposed model uses only readily available material parameters. The proposed damage growth model is extended into the stochastic domain by considering fluctuations in the free energy, and closed-form solutions of the resulting stochastic differential equation are obtained in each of the three cases mentioned above. A reliability analysis of a ring-stiffened cylindrical steel shell subjected to corrosion, accidental pressure and temperature is performed. 



\section{Contents}

Abstract

Contents $\quad$ v

List of Figures $\quad$ ix

List of Tables $\quad$ xi

Executive Summary

Acknowledgments $\quad$ Xv

1 Introduction 1

1.1 Statement of Problem .......................... 1

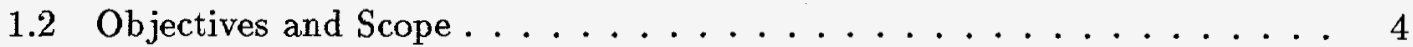

1.3 Organization of Report . . . . . . . . . . . . . . . . 4

2 Failure Mechanisms in Metal Structures 7

2.1 Plastic Deformation . . . . . . . . . . . . . . 7

2.2 High-temperature Creep . . . . . . . . . . . . . . . . . 9

2.3 Fatigue and Fracture . . . . . . . . . . . . . . . 10

2.3 .1 Fracture ......................... 10

2.3 .2 Fatigue ......................... 12

2.3.3 Randomness in Fatigue Crack Growth . . . . . . . . . . 15

2.4 Corrosion . . . . . . . . . . . . . . . . . . 17

2.4.1 Overview and Types of Corrosion . . . . . . . . . . . 17

2.4.2 Mechanistic and Statistical Models of Corrosion . . . . . . . 18

2.5 Appraisal of Existing Methods . . . . . . . . . . . . . . . . . 19

2.5.1 Fatigue, Fracture and Creep . . . . . . . . . . . 20

2.5 .2 Corrosion . . . . . . . . . . . . . . . 21

2.5.3 The "Damage" State Variable . . . . . . . . . . . . . 22

3 CDM-based Approaches to Modeling Structural Deterioration 25

3.1 Introduction . . . . . . . . . . . . . . . 25

3.2 Fundamentals of Damage Mechanics ................ 26

3.3 Review of Existing CDM-based Methods . . . . . . . . . . . . . 29

3.3.1 Kinetic Equation Method . . . . . . . . . . . . . . 29 
3.3.2 Thermodynamic Potential Method .............. 30

3.4 Thermodynamic Foundations of Damage Growth . . . . . . . . . . . 33

3.4.1 Thermodynamically Irreversible Processes ........... 33

3.4 .2 The Dissipation Rate ................ . . 34

3.5 Damage Growth in Deformable Bodies . . . . . . . . . . . . 36

4 A Unified Model for Isotropic Damage Growth

4.1 The Isotropic Damage Growth Model . . . . . . . . . . . . . . . . . 45

4.2 Ductile Deformation Damage . . . . . . . . . . . . . . . . 50

4.2 .1 Damage Growth Models . . . . . . . . . . . . . . 50

4.2.2 Model Validation and Sensitivity Study ........... 54

4.3 Creep Damage . . . . . . . . . . . . . . . . 57

4.3.1 Damage Growth Predictions . . . . . . . . . . . . . . 57

4.3.2 Verification and Prediction of Trends . . . . . . . . . . . . 59

4.4 Fatigue Damage ... . . . . . . . . . . . . . 61

4.4 .1 Damage Analysis . . . . . . . . . . . . . . . 61

4.4.2 Verification of the Proposed Model . . . . . . . . . . . 66

4.4.3 Prediction of Load Sequencing Effects ............ 67

4.5 Closure . . . . . . . . . . . . . . . . . . . . 69

5 Stochastic CDM and Structural Reliability 95

5.1 Introduction . . . . . . . . . . . . . . . . 95

5.2 Fundamentals of Stochastic Differential Equations . . . . . . . . . 96

5.3 Development of CDM-based Stochastic Models . . . . . . . . . . . 102

5.3.1 Review of Previous Work . . . . . . . . . . . . . . 102

5.3.2 Stochastic Isotropic Damage Growth . . . . . . . . . . . . 103

5.4 Stochastic Ductile Damage . . . . . . . . . . . . . . . 107

5.4.1 Equation and Stochastic Characterization .......... . 107

5.4.2 Failure Probability in Random Ductile Damage Growth . . . . . . 110

5.4.3 Model Validation, Sensitivity and Reliability Studies . . . . . . 111

5.5 Stochastic Creep Damage . . . . . . . . . . . . . . . . . 114

5.5.1 Equations and Stochastic Characterization ........... 114

5.5.2 Model Verification and Studies in Reliability . . . . . . . . . 116

5.6 Stochastic Fatigue Damage . . . . . . . . . . . . . . . . 119

5.6.1 Equations and Stochastic Characterization . . . . . . . . 119

5.6 .2 Validation and Studies in Reliability . . . . . . . . . . . . 122

5.7 Closure . . . . . . . . . . . . . . . . . . . . 124

6 Condition Assessment of Aging Metal Structures 141

6.1 Introduction: Description of Structure . . . . . . . . . . . . . 141

6.2 Reliability under Corrosion . . . . . . . . . . . . . . 143

6.2.1 Effect of Corrosion . . . . . . . . . . . . . . 143

6.2.2 Numerical Examples and Sensitivity Analyses . . . . . . . . . . . 149

6.3 Reliability under Thermal Creep Damage . . . . . . . . . . . . . . . 152

6.3.1 Creep Damage at Elevated Temperatures . . . . . . . . . 153

6.3 .2 Numerical Example ... . . . . . . . . . . . 155 
7 Conclusions and Recommendations

7.1 Summary of Research and Findings . . . . . . . . . . . . . . . 169

7.2 Suggestions for Future Work . . . . . . . . . . . . 173

A Crack-tip Plasticity and Propagation Life $\quad 175$

Bibliography 



\section{List of Figures}

3.1 The damage variable . . . . . . . . . . . . . . . . . 41

3.2 Experimental determination of damage . . . . . . . . . . 42

3.3 State variables and energy exchange for a closed system . . . . . . . 43

4.1 Elastic and plastic components of total strain . . . . . . . . . . . 79

4.2 Ductile damage growth in SAE 1035 steel . . . . . . . . . . . . 80

4.3 Ductile damage growth in 2024-T3 aluminum . . . . . . . . . . . 81

4.4 Ductile damage growth in AISI 4130 steel . . . . . . . . . . . . . 82

4.5 Ductile damage growth in INCO 718 alloy . . . . . . . . . . . 83

4.6 Sensitivity of proposed ductile damage growth model to $E$. . . . . . . 84

4.7 Sensitivity of proposed ductile damage growth model to $K \ldots \ldots$. . . . 84

4.8 Sensitivity of proposed ductile damage growth model to $M \ldots \ldots$. . . 85

4.9 Sensitivity of proposed ductile damage growth model to $\sigma_{f} \ldots \ldots$. . 85

4.10 Sensitivity of proposed ductile damage growth model to $\epsilon_{0} \ldots \ldots$

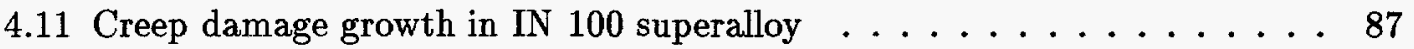

4.12 Effect of applied stress on predicted creep damage growth in ASTM A36 steel at constant temperature. . . . . . . . . . . . . . 88

4.13 Effect of temperature on predicted creep damage growth in ASTM A36 steel at constant stress . . . . . . . . . . . . . . 88

4.14 Effect of $D_{0}$ on predicted creep damage growth in ASTM A36 steel at constant stress ......................... 89

4.15 Predicted creep damage growth in type 316 stainless steel under different stress levels at constant temperature . . . . . . . . . . . 90

4.16 Predicted and experimental strain-controlled fatigue damage growth in type 316 stainless steel . . . . . . . . . . . . . . . . 91

4.17 Predicted and experimental fatigue damage growth in A106-Gr B steel at $288^{\circ} \mathrm{C}$ in air . . . . . . . . . . . . . . . . . . 92

4.18 Predicted and experimental fatigue damage growth in SAE 4340 steel at room temperature in air . . . . . . . . . . . . . . 93

4.19 Effect of load sequencing on damage growth in stress-controlled fatigue of SAE 4340 steel . . . . . . . . . . . . . . . . . . . . . . 94

4.20 Predicted failure (i.e., crack initiation) in two-level stress-controlled fatigue cycling of SAE 4340 steel . . . . . . . . . . . . . 94

5.1 Stochastic ductile damage growth in 2024-T3 Aluminum under deterministic initial condition and material parameters . . . . . . . . 125 
5.2 Effect of randomness in material parameters and initial damage on stochastic ductile damage growth in 2024-T3 Aluminum . . . . . . . . . . . 126

5.3 Effect of correlation among the random initial condition and material parameters on stochastic ductile damage growth in 2024-T3 Aluminum. . . . 127

5.4 Effect of noise intensity on stochastic ductile damage growth in 2024-T3 Aluminum, with moderate correlation among the random variables . . . 1248

5.5 Effect of noise intensity on stochastic ductile damage growth in 2024-T3 Aluminum, with perfect correlation among the random variables. . . . . 129

5.6 Probability of negative damage increment in the proposed model . . . . 130

5.7 Failure probability, sample paths of damage and probability distribution of $D_{c}$ and $\epsilon_{f}$ in 2024-T3 Aluminum . . . . . . . . . . . 131

5.8 Dependence of creep damage on noise intensity in A36 steel stressed to

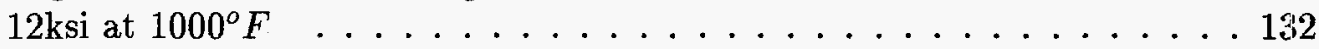

5.9 Statistics of damage, failure probabilities in creep of A36 steel stressed to

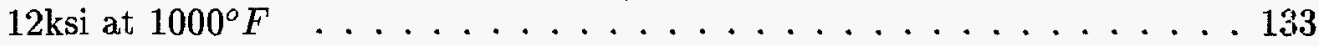

5.10 Sample paths of creep damage (under Stratonovich interpretation) in type 316 stainless steel stressed to $199 \mathrm{MPa}$ at $1100^{\circ} \mathrm{F} \ldots \ldots \ldots 134$

5.11 Sample paths of creep damage (under Ito interpretation) in type 316 stainless steel stressed to $199 \mathrm{MPa}$ at $1100^{\circ} \mathrm{F} \ldots \ldots \ldots \ldots . \ldots \ldots$

5.12 Statistics of damage and failure probabilities (under Stratonovich interpretation) in creep of type 316 stainless steel stressed to $199 \mathrm{MPa}$ at $1100^{\circ} \mathrm{F} 136$

5.13 Statistics of damage and failure probabilities (under Ito interpretation) in creep of type 316 stainless steel stressed to $199 \mathrm{MPa}$ at $1100^{\circ} \mathrm{F} \ldots \ldots . .137$

5.14 Random fatigue damage growth in A106-B carbon steel at $288^{\circ} \mathrm{C}$ in air .138

5.15 Random number of cycles to failure (fatigue crack initiation) in A106-B carbon steel at $288^{\circ} \mathrm{C}$ in air . . . . . . . . . . . . . . . 139

5.16 Probability of failure (fatigue crack initiation) in A106-B carbon steel at $288^{\circ} \mathrm{C}$ in air . . . . . . . . . . . . . . 140

6.1 Poisson arrival rates of accidental pressures with $\lambda_{0}=0.0001 / y r \ldots 159$

6.2 Cumulative failure probability (CFP) of ring-stiffened nuclear power plant (NPP) steel containment subjected to internal pressurization, severe cor-

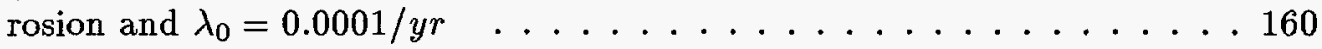

6.3 CFP of ring-stiffened NPP steel containment subject to internal pressurization in absence of corrosion with $\lambda_{0}=0.0001 / y r \ldots \ldots 161$

6.4 Effects of corrosion rate on reliability with and without aging . . . . . 162

6.5 Effect of setting initiation time equal to its mean and ignoring initiation

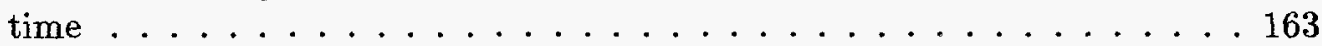

6.6 Effect of setting corrosion rate equal to its mean under severe corrosion . 164

6.7 Effect of setting corrosion exponent equal to its mean under severe corrosion 165

6.8 Sample path of random creep damage growth in steel containment shell . 166

6.9 Cumulative failure probability in creep damage accumulation . . . . . 167 


\section{List of Tables}

4.1 Room temperature monotonic material properties of various alloys . . . 71

4.2 Experimental and predicted critical damage in ductile deformation . . . . 72

4.3 Creep strain growth law parameters for ASTM A36 steel . . . . . . . . 73

4.4 Creep strain growth law parameters for type 316 stainless steel . . . . . . 74

4.5 Experimental and predicted failure stresses due to creep in ASTM A36 steel using Fields and Fields data . . . . . . . . . . . . . . 75

4.6 Experimental and predicted failure stresses due to creep in ASTM A36 steel using Harmathy data . . . . . . . . . . . . . . . 76

4.7 Experimental and predicted failure times (hours) due to creep in type 316 stainless steel. . . . . . . . . . . . . . . . 77

4.8 Cyclic material properties of various alloys . . . . . . . . . 78

6.1 Nominal (uncorroded) dimensions, SA 516/70 material properties, and load and resistance statistics for a typical NPP steel containment. . . . 156

6.2 Different cases of uniform corrosion of the NPP steel containment . . . 157

6.3 Different cases of aging of safety-related equipment in the NPP steel containment ......................... 157

6.4 Conditional failure probabilities due to creep damage growth in NPP containment shell . . . . . . . . . . . . . . . . 158 



\section{Executive Summary}

Steel containments and liners in nuclear power plants (NPPs) may exhibit structural aging effects, caused by processes that depend on the operating environment, service or environmental loads, and unforeseen or accidental events. Aging may cause the strength or stiffness of a structure to degrade during its service life to the point where there may be a loss of function or integrity, leading to costly maintenance, repair, or replacement. Load-induced inelastic deformation, corrosion, elevated temperature creep, and fatigue are examples of damage accumulation mechanisms associated with aging in steel structures. It is essential to be able to quantify structural deterioration to perform condition assessments of an existing structure, to estimate its residual strength and remaining service life, and to develop appropriate strategies for maintaining it in a safe and functional condition.

The initial stages of structural deterioration often occur without perceptible or visible manifestation. By the time that damage reaches the point where it is detectable by the customary nondestructive evaluation methods, a significant fraction of the remaining service life or residual strength may already have been exhausted, and costly repairs may be required as a condition of continued service. However, the existing methods for modelling structural deterioration usually require a measurable flaw to be applicable (e.g., Paris Law); and those that predict the service-life of a structure often cannot provide estimates of residual strength while the structure is still in service (e.g., S-N curve). Moreover, existing methods for assessment of damage accumulation are largely empirical in nature, expressing damage either as a function of the elapsed time normalized by the failure time or through regression analyses of experimental data on damage. These approaches are heavily reliant on experimental data, and frequently lack a strong basis in structural mechanics. Accordingly, the extrapolation of these experimental, empirical relationships to structural service conditions raises numerous issues regarding the validity of scaling from the laboratory to the prototype. Finally, structural damage growth is intrinsically random, even under well-controlled laboratory conditions. Existing approaches often encapsulate uncertainty in the damage accumulation process by treating the experimental constants simply as random variables. Such approaches may not account properly for the underlying causes of the randomness.

The research described in this report is in support of the Steel Containments and Liners Program being conducted for the U.S. Nuclear Regulatory Commission under overall management by Lockheed-Martin Energy Research Corporation, Oak Ridge National Laboratory. The goals of the research are to identify mathematical models from principles of mechanics to evaluate structural degradation, to recommend statisticallybased sampling plans for nondestructive evaluation (NDE) of complex structures, and to identify methods to assess the probability that containment or liner capacity has not degraded, or will not degrade during a future service period.

Rational methodologies for analyzing damage growth in steel containments and lin- 
ers can be developed within the context of the relatively new field of classical mechanics known as continuum damage mechanics (CDM), coupled with structural reliability analysis principles that take uncertainties in loading, in initial material strength and stiffness, and in their subsequent deterioration into account. CDM deals with the aggregate effects of micro-structural defects and expresses them in terms of quantities that are observable at the macro-structural level, e.g., changes in the elastic modulus of the material or stiffness of the component. CDM is well-suited for tackling several of the damage growth processes affecting steel structural systems with a significant reduction from current levels of empiricism. CDM-based damage growth laws arise out of the classical laws of thermodynamics in a mathematically elegant way. They can be extended naturally in the stochastic domain with the assumption that randomness in damage growth is caused by spatial/temporal fluctuations in energy and in material properties.

The report begins with a review of current approaches for analyzing damage accumulation due to corrosion, plastic deformation and creep, and fatigue and supporting experimental data in Chapters 1 and 2. Fundamental notions of cumulative damage mechanics, its thermodynamic foundations, and equations of isotropic damage growth for ductile deformation, high-temperature creep, and fatigue are presented in Chapters 3 and 4 . The predictions are validated with experimental data. The deterministic CDM formulation is extended to the stochastic domain in Chapter 5, where a random noise term reflecting local fluctuations in material properties and energy dissipation mechanisms is introduced. The stochastic differential equations that predict uncertainty in damage accumulation are developed, and the predictions are compared with available experimental data. An application of various damage growth models to reliability-based condition assessment of a ring-stiffened steel cylindrical shell, typical of a containment subjected to internal pressurization and elevated temperatures, is presented in Chapter 6 . Chapter 7 contains a summary of the major findings of the research, and recommendations for additional studies. A comprehensive bibliography on structural damage and deterioration due to corrosion, excessive ductile deformation, elevated temperature creep and fatigue concludes the report. 


\section{Acknowledgment}

This research was conducted as part of the U.S. Nuclear Regulatory Commission's Steel Containments and Liners Program, supported by Lockheed-Martin Energy Research Corporation under Contract No. 19X-SP638-V. This support and the technical advice provided by Dr. Dan Naus of Oak Ridge National Laboratory and Mr. Wallace E. Norris of the U.S. Nuclear Regulatory Commission, are gratefully acknowledged. Thanks also are due to Mr. Omesh Chopra of Argonne National Laboratory for kindly making available some of the fatigue data used in validating the theoretical damage models in this report. 


\section{Chapter 1}

\section{Introduction}

\section{$1.1 \quad$ Statement of Problem}

Steel is widely used in the construction and manufacturing industries. It finds its use in a wide variety of structural engineering applications: building structures, power plants, bridges, ships, aircraft and automobiles. Its chief advantages are a high strength-toweight ratio, temperature resistance and ductile behavior between initial yielding and fracture. Steel is amenable to fabrication in a variety of shapes and components. In addition, uncertainties in the strength, stiffness and section properties of a steel component can be substantially reduced by good quality control in the manufacturing process.

However, steel structures are susceptible to aging (deterioration in strength and stiffness) by various processes depending on the operating environment and service loading conditions. Corrosion, high-temperature creep and fatigue crack growth are examples of such deleterious processes. The effects of these processes may accumulate within the structure over time, and may cause failure under design conditions or lead to costly repair. Structural failures create the potential for loss of human life (e.g. collapse of a building), infringe on public safety (e.g., failure of a nuclear power plant containment causing radiation leakage), and have severe economic and social consequences. Even the temporary suspension of operations of an important structure to facilitate repairs (e.g., the closing down of a bridge) can impose a sizeable burden on the local economy.

It is essential to be able to quantify structural deterioration and estimate the residual strength and service-life of a degrading structure so that it can be maintained economically while remaining in service. Considering the need to optimize maintenance expenditures, the methods of estimating structural deterioration should ideally be able to detect/predict unacceptable levels of damage early enough, so that costly and disruptive repairs can be avoided. The need for reliable methods of predicting structural damage growth and estimating residual strength gains further relevance with the fact that aging of the infrastructure is a widespread phenomenon and one that is becoming more acute 
every year. For example, by one estimate (Schweitzer, 1987), the cost of corrosion in the United States, taking maintenance, use of special materials and failure of equipment or infrastructure into account, is 80 billion dollars annually.

Although the ultimate effect of deterioration processes in steel structures is to compromise structural performance, the physical manifestations of structural degradation can be as varied as the processes which cause them. In many situations (like general corrosion $^{1}$ ), the growth of structural damage is gradual and stable and leaves sufficient time to react and decide on the best remedial measure. In some situations, however, the effect of structural decay (for example creep) is not easily detectable until it has progressed substantially. There also are situations where the growth rate of damage is highly nonlinear (e.g., high-cycle fatigue), and over a major portion of its service life the structure remains essentially defect-free. In such cases, insufficient or limited time might remain after the first detection of damage to take remedial actions.

Among the various aging processes, perhaps the most visible and easily detectable is general corrosion which can be identified by changes in texture, color and thickness of a steel surface. However, another form of corrosion, called pitting corrosion, which can penetrate a section (deemed as failure for fluid and pressure-retentive structures such as pipelines and pressure vessels), can be extremely difficult to detect as the pits are small in diameter and are often hidden under general corrosion products. The growth rate of pits moreover can be orders of magnitude higher than that of general corrosion in a given environment.

The accumulation of fatigue damage in steel components causes the formation and subsequent growth of cracks, which may finally become unstable and lead to failure. The time to crack nucleation is often the most important factor in the total fatigue life of a structure. Once formed, the detection and correct sizing of fatigue cracks are crucial in determining the residual life, but accurate identification and measurement of cracks are not always achieved in practice.

Creep straining is the result of sustained loading in the presence of high temperature which may lead to fracture if loaded long enough, even at stresses much below the ultimate strength at that temperature. Creep failure can occur without necking and with little warning. There are yet other processes of structural decay, like embrittlement of steel (caused by radiation and hydrogen ions), which also do not have "visible" manifestations but can potentially cause failure of the structure.

The existing models of structural degradation are mostly empirical in nature. They either use an a posteriori approach, expressing damage as a function of the elapsed time normalized by the failure time (as in Miner's rule in fatigue); or fit suitable functions to the abserved data (e.g., S-N curve for fatigue failure) without explaining their mechanistic bases. The shortcoming of the first approach is obvious: it requires knowledge

\footnotetext{
${ }^{1}$ Diferent types of corrosion, high-temperature creep, fatigue and plastic deformation are discussed in detail in Chapter 2.
} 
of the time or number of cycles to failure of an existing structure which is not always forthcoming. The latter approach generally involves extensive testing, and often requires a measurable flaw (e.g., a crack size) which restricts the situations where such models may be applied. Moreover, many of the above approaches lack the ability of providing an estimate of residual strength, the knowledge of which is essential in evaluating the safety of a degrading structure.

Rational models to analyze damage growth are therefore needed that can not only provide estimates of residual strength and remaining service life of a structure based on the knowledge of its service conditions, but can do so even when the structure does not show any perceptible sign of decay. As mentioned above, this is especially important when damage either grows below the detectability threshold ${ }^{2}$ and accelerates to failure by the time it is detected, or consumes a major part of the service life to initiate and grows swiftly thereafter.

Structural damage growth is intrinsically a random phenomenon. Indeed, even under tightly controlled laboratory test conditions, damage growth data are found to exhibit significant scatter. Mathematical models for predicting damage growth should therefore be able to capture the stochastic nature of the process - providing at the least an estimate of the variability or uncertainty in the prediction. The empirical equations (Miner's rule and S- $\mathrm{N}$ curve) discussed above are not able to account for the inherent randomness of a given damage growth process unless they are "randomized" with the help of additive or multiplicative stochastic noise. Such modifications do not relate to the underlying cause of the randomness.

Continuum damage mechanics (CDM), a relatively new development out of classical continuum mechanics, deals with the aggregate effects of micro-structural defects and expresses them in terms of macro-level quantities - for example, a change in the modulus of elasticity. It is shown in the subsequent chapters that CDM is well suited to tackle several of the damage growth processes described above with a significantly lesser degree of empiricism. CDM is especially suitable for estimating damage growth that occurs prior to the formation of macro-defects. Since it relates structural damage to physically measurable material properties, CDM can provide estimates of residual strength of a damaged structure at any arbitrary time in service. Furthermore, as will be shown subsequently, CDM-based damage growth laws arise out of the thermodynamic properties of damage accumulation in a mathematically elegant way. They can naturally be extended into the stochastic domain under the assumption that randomness in damage growth is caused by spatial and/or temporal variations in energy and material properties.

In view of the random damage growth in structures, coupled with uncertainties in the loading processes and the environment, it is appropriate to take a probability-based approach to analysis of structural safety using CDM. The determination of the timedependent reliability and hazard functions of a structure (in a random environment

\footnotetext{
${ }^{2}$ The minimum detectable damage is a function of the skill of the inspector and instrument sensitivity.
} 
subjected to random loading) can give a clear understanding of when in its service-life the structure is likely to slip out of the acceptable safe domain or when an inspection/repair should be scheduled to maintain it in service with adequate safety. A stochastic CDM approach to time-dependent reliability is also helpful in deciding the service-life extension of an existing structure or ascertaining whether an existing structure can withstand a different operating or service environment than that for which it originally was designed.

\subsection{Objectives and Scope}

The objectives of this research are to formulate rational damage growth laws for steel structures that model mechanisms of structural damage accumulation due to gross inelastic deformation, creep, fatigue and corrosion while taking into account the randomness in the processes; to estimate remaining service-life or residual strength and uncertainties in these key decision parameters using the damage growth laws and stochastic models; and to ascertain the reliability of a structure subject to one or more of these mechanisms.

\subsection{Organization of Report}

A background on the four different damage-causing processes considered in this report, namely corrosion, fatigue, plastic deformation and creep, is presented in Chapter 2 . It includes the existing mathematical models and available statistical treatment of the damage-causing processes. A critical appraisal of the existing methods of quantifying structural deterioration and finding the residual strength/remaining life of a degrading structure concludes Chapter 2.

Chapter 3 introduces the fundamentals of continuum damage mechanics (CDM). A review of the existing CDM-based damage growth laws is presented. The method proposed in this research is then developed. The thermodynamic foundations of damage are discussed, and variational results for deformable bodies arising therefrom are presented.

Chapter 4 concentrates on isotropic damage growth in deformable bodies. It derives equations of damage growth for ductile deformation, high-temperature creep and fatigue from the fundamental thermodynamic principles presented in Chapter 3 . These growth models are compared with experimental results and behavioral trends published in the literature. Sensitivity studies on the different material parameters in the models are also presented.

Chapter 5 extends the models developed in Chapters 3 and 4 into the stochastic domain. Existing CDM-based models of stochastic damage growth are reviewed at the beginning of this chapter. Some basic concepts of stochastic differential equations required for the stochastic CDM approach in this report are presented. The intrinsic material and energy variations that cause randomness in the state of damage and its 
growth rate are shown to lead to a stochastic differential equation of damage growth, which is then solved to obtain the random damage growth laws in ductile deformation, creep and fatigue. Finally, damage accumulation predicted by the proposed models is compared with available existing experimental data.

Chapter 6 presents an application of the damage growth models developed in the previous chapters. The reliability-based condition assessment of a steel ring-stiffened cylindrical shell (typical of a nuclear power plant containment) subject to internal pressurization and high temperature is performed. Several different scenarios of loading and aging are considered and the parameters having potentially significant effects on the life-time reliability of the structure are identified.

Chapter 7 summarizes the major findings from this research and recommends areas for additional study. 



\section{Chapter 2}

\section{Failure Mechanisms in Metal Structures}

The four major mechanisms of degradation of steel structures, namely plastic deformation, high-temperature creep, fatigue and corrosion are discussed in this chapter. The physical mechanisms governing these different processes and their available mathematical and statistical models are examined first. Following that, a critical appraisal of the existing methods of quantifying structural damage and estimating the residual strength or remaining service of a degrading structure is presented.

\subsection{Plastic Deformation}

The most common way to characterize a metal for structural purposes is through its monotonic (engineering) stress-strain curve. The deformation of a material upon application of load may, in general, have a time-dependent component ${ }^{1}$ in addition to the more common instantaneous (depending only on the applied load) component. In this section, the instantaneous component of deformation will be discussed.

Upon application of load, the strain response of a structure can be separated into two constituents: the fully-recoverable elastic strain and the permanent plastic strain. For lower values of stresses, plastic strain is relatively small and may even be completely absent (at least from a macroscopic viewpoint). Some materials show a distinct yield point, below which all the strain is recoverable. A metal undergoes strain hardening with accumulation of plastic strain if loaded beyond the yield point. For many metals (e.g., SAE 4340) a distinct yield point does not exist, but as the yield strength, $\sigma_{y}$, is a widely-used mechanical property, it is usually defined for such materials to be the stress corresponding to either a $0.2 \%$ plastic strain or a $0.5 \%$ elongation.

\footnotetext{
${ }^{1}$ For example, creep straining at high temperatures, which is discussed in Sec 2.2.
} 
Metals and alloys can be broadly classified as ductile or brittle according to their behaviour before failure. Ductile materials sustain a large plastic deformation prior to fracture; brittle materials have relatively little deformation even at ultimate loads and fracture suddenly without much prior indication. For example, ductile cast iron (A536, 65-45-12) has a failure strain of around $15 \%$ at room temperature, whereas the corresponding value for SAE 308 cast aluminum is only $0.9 \%$ (Dowling, 1993). Temperature plays an important role in the extent of ductile behavior of a metal: a material that is ductile at room-temperature may become brittle at low temperatures. Theoretically, a large hydrostatic tension would cause a normally ductile material to exhibit brittle failure. An originally ductile material may be cold worked to suit particular applications a process which gives it a higher yield point but takes away some of its ductility. In general, ductility is a desirable property in a structural material, as it allows the structure to provide sufficient warning in the event of an impending collapse.

The source of elastic and plastic deformations in a metal can be traced to the structure of the material in the atomic scale. In an idealized crystal lattice, the atoms are displaced from their equilibrium positions upon the application of an external load. The resulting strain is elastic if the atoms return to their original equilibrium positions once the load is withdrawn. On the other hand, a large enough load causes one plane of atoms in the lattice to slide over another, and when the load is withdrawn the atoms come to rest in new equilibrium positions in the lattice. This is called slip and manifests itself as plastic deformation in the macroscale (Collins, 1981). Plastic deformation is often concentrated in bands called slip bands. Other microscopic mechanisms causing plastic deformation are twinning ${ }^{2}$ and grain boundary sliding.

Mechanical properties (like tensile strength and fracture ductility) as well as stressstrain curves are frequently available in terms of engineering stresses and strains, $S$ and $e$ (engineering quantities are obtained using the original dimensions of the specimen). The corresponding true quantities, $\sigma$ and $\epsilon$ (which are defined with regard to the deformed dimensions) can be obtained using the following relations:

$$
\sigma=S(1+e), \quad \epsilon=\ln (1+e),
$$

Under uniaxial loading, the relation between the true elastic strain $\left(\epsilon_{e}\right)$ and true stress $(\sigma)$ is idealized as linear for most metals, while the true plastic strain $\left(\epsilon_{p}\right)$ and true stress relation is often described by a power law. Assuming that the total strain, $\epsilon$, is the sum of its two components, one obtains the widely used Ramberg-Osgood law under monotonically increasing uniaxial loading (Dowling, 1993):

$$
\begin{aligned}
\epsilon & =\epsilon_{e}+\epsilon_{p} \\
& =\frac{\sigma}{E}+\left(\frac{\sigma}{K}\right)^{M}
\end{aligned}
$$

\footnotetext{
${ }^{2}$ Twinning involves rotation of atoms in the lattice and occurs in crystals that do not permit slip deformation. Deformation due to twinning is very small compared to that due to slip (Stouffer and Dame, 1996).
} 
where $E$ is the elastic modulus, $K$ is the strain hardening modulus and $M$ is the strainhardening exponent. An estimate of the strain-hardening exponent can be obtained from the ultimate engineering strain:

$$
M=\frac{1}{\ln \left(1+e_{u}\right)}
$$

where the subscript $u$ (for ultimate) refers to the highest point on the $S-e$ curve.

In the above discussion of monotonic tensile properties, quasi-static loading was implicitly assumed. These properties, however, can be quite sensitive to the rate of load application, and are often qualified as strain-rate effects. In general it is observed that: with increasing strain rate, the strength properties (namely, yield strength, elasticity modulus, ultimate strength) increase, but ductility and strain-hardening rate decrease (Dowling, 1993; Boyer, 1987).

Experimental values of material parameters (for a given grade of material) often exhibit significant scatter (e.g., Cherry, $1996^{3}$ ). While a part of the scatter may be due to measurement errors and different conditioning of the same nominal material grade, the scatter also arises out of intrinsic material variabilities. Randomness in material parameters may have a significant impact on structural degradation and failure, and random damage growth models have been obtained under the equal rank hypothesis by treating the material parameters in an existing deterministic model as random variables.

\subsection{High-temperature Creep}

In the previous section, the time-independent effects of loads on a structure were considered. The resultant deformations depended only on the magnitude of the applied loads, and the structural response did not change with time as long as the load was held constant. The room-temperature behaviour of most metals usually fall in this category.

Creep strain, on the other hand, is time-dependent. Even when the load is held constant and the instantaneous elastic and plastic strains have been realized, the specimen may continue to strain further with time: this continuation of straining is defined as creep. Creep is heavily temperature-dependent. Few metals exhibit noticeable creep under design loading conditions at room temperature. However, at temperatures above one-third to two-thirds of the absolute melting temperature, the creep rate becomes significant, and the specimen may fail even at load levels much below the design stress (at that particular temperature), if loaded long enough (Dowling, 1993). Examples of melting temperatures of some materials are: $1813 \mathrm{~K}\left(2800^{\circ} \mathrm{F}\right)$ for iron, $1723 \mathrm{~K}\left(2640^{\circ} \mathrm{F}\right)$ for stainless steel, $1673 \mathrm{~K}\left(2550^{\circ} \mathrm{F}\right)$ for steel and $933 \mathrm{~K}\left(1220^{\circ} \mathrm{F}\right)$ for aluminum alloys. Creep rupture typically occurs "without necking and without warning" and is like brittle failure

\footnotetext{
${ }^{3}$ Based on test data from 489 specimens of pressure vessel grade steel, Cherry (1996) reports coefficients of variation of $12 \%$ for yield strength, $7 \%$ for ultimate strength and $22 \%$ for failure strain.
} 
in this regard. No significant correlation exists between room temperature mechanical properties of a material and its creep behavior (Collins, 1981, p. 437). The nature of creep deformation is different from that of tensile and fatigue deformations which occur mainly due to slip. Creep occurs due to the diffusion of atoms and vacancies through the crystal lattice and along grain boundaries (Stouffer and Dame, 1996).

Creep straining, starting from initiation to failure, can be assigned three stages: (i) primary creep, just after initiation when the rate is very high but falls off rapidly to (ii) secondary creep, where the rate is constant (also called steady-state creep) and eventually leading to (iii) tertiary creep, in which the rate accelerates ultimately leading to rupture. Usually, the secondary stage covers the major portion of the creep life.

The uniaxial creep strain as a function of $t$ (time), $\sigma$ (uniaxial stress) and $T$ (temperature) is commonly expressed as:

$$
\epsilon_{\mathrm{cr}}=A(T) \sigma^{n} t^{\phi}
$$

which is sometimes referred to as the Bailey-Norton law, in which $A, n, \phi$ are material constants. For steady-state creep, $\phi=1$. In addition, if $n=1$, we have Newtonian viscosity. The explicit dependence on temperature can be removed by introducing the temperature-compensated time, $\theta$, (Harmathy, 1967) from the Arrhenius equation for thermal activation of creep:

$$
\theta=\int_{0}^{t} \exp \left(-\frac{\Delta H}{R T}\right) d t^{\prime}
$$

such that

$$
\begin{aligned}
\frac{d \epsilon_{c}}{d \theta} & =f(\sigma) \\
\frac{d \epsilon_{c}}{d t} & =\frac{d \epsilon_{c}}{d \theta} \frac{d \theta}{d t}
\end{aligned}
$$

where $\Delta H$ is the activation energy of creep and $R$ is the universal gas constant. For example, the quantity $\Delta H / R$ is equal to $70000^{\circ} \mathrm{R}$ for A36 steel (Harmathy, 1967) where ${ }^{\circ} \mathrm{R}$ is degree Rankine.

A set of experiments showing randomness in all three stages of creep damage growth is available in Garofalo et al (1961) ${ }^{4}$.

\subsection{Fatigue and Fracture}

\subsubsection{Fracture}

Fracture is the separation of a member into two pieces due to application of loads. A structural metal contains voids and inclusions at the microscopic level. Along with these

\footnotetext{
${ }^{4}$ Garofalo et al (1961) report the times to (i) the end of primary creep stage, (ii) the end of secondary stage and (iii) failure, in type 316 stainless steel at constant temperature and stress.
} 
microstructural "defects", a component may contain geometric discontinuities (weld undercuts, rivet holes), sudden changes in section required by design, and local defects (cracks in a weldment or corrosion loss) that accumulate as a result of service conditions or faulty fabrication, all of which raise the stress state locally. These elevated local stresses may cause failure when the nominal applied stress is still in a "safe" range. Quantities which are used to evaluate the effects of such stress raisers include the stress concentration factor $K_{t}$, stress intensity factor $K$, strain energy release rate $G$ and $J$ integral. For example, at the edge of a circular hole in an infinite plate under remote tensile loading $\sigma$, the stress concentration factor $K_{t}=3$; and the mode I stress intensity factor at the tip of a center crack of length $2 a$ in an infinite plate loaded similarly is $K_{I}=\sigma \sqrt{\pi a}$. Among the material properties which signify strength against fracture is the plane strain fracture toughness ${ }^{5}, K_{I_{c}}$. Materials with high yield strength generally have low fracture strength, and vice versa (Dowling, 1993). Usually, materials with $K_{I_{c}}<5 M P a \sqrt{m}$ are brittle, and those with $K_{I_{c}}>20 M P a \sqrt{m}$ are ductile, e.g., for carbon steel, the value typically is $K_{I_{c}}=140 M P a \sqrt{m}$ whereas for soda-lime glass it is $0.76 M P a \sqrt{m}$.

The concept of the stress intensity factor $K$ is confined to the field of Linear Elastic Fracture Mechanics (LEFM) in which it is assumed that Hooke's law is valid throughout the material, including near the crack-tip (Kanninen and Popelar, 1985) ${ }^{6}$. Such an idealization is acceptable if the material is brittle, but LEFM may be inadequate for ductile materials because the crack-tip stress cannot exceed $\sigma_{y}$ and the plastic region around the crack-tip simply spreads with an increase in the far-field load. Though such behavior ideally comes under the purview of Elastic-Plastic Fracture Mechanics (EPFM) ${ }^{7}$, the wide popularity and the sound analytical basis of LEFM have led to several modifications (for example the plastic zone correction factor to define the effective stress intensity factor) to make it applicable to a wider array of real materials. Since real materials are composed of discrete grains, voids and inclusions which manifest as discontinuities at the micro-structural level, LEFM-based quantities should not be applied at the scale where the continuum approach breaks down - usually in the domain of small and short cracks (Dowling, 1993). At this scale, micro-structural features like the average crystal grain size and the average particle spacing may become the dominant factors in their growth and closure phenomena.

\footnotetext{
${ }^{5}$ The fracture toughness, $K_{c}$, in general depends on the temperature, loading rate and thickness of the member. The plane strain fracture toughness gives the lowest possible value of $K_{c}$, and is considered the most critical case.

${ }^{6} \mathrm{~A}$ direct consequence of this is the existence of extremely intense stress fields (which theoretically reach infinity) around the crack-tip irrespective of the magnitude of the far-field loading.

${ }^{7}$ In EPFM, the most widely applied concept is the $J$-integral introduced by Rice in 1968 , which can be used to solve a variety of crack-tip plasticity problems (Kanninen and Popelar, 1985). In the absence of crack-tip plasticity, $J$ becomes equal to $K^{2} / E$, and thus serves to unify LEFM and EPFM.
} 


\subsubsection{Fatigue}

In the foregoing discussion on failure of materials, static loading was implicitly assumed. The situation is different in the presence of repeated or cyclic loading (which may be due to service cycling, thermal cycling etc). This reversal of load may cause movements of dislocations at the microscale leading to the formation of surface grooves and ridges; or may form vacancies on slip planes that lead to holes in the lattice structure (Collins, 1981). This microstructural damage manifests as fatigue in the metal, causing cracks to nucleate in an originally defect-free member, and subcritical cracks to grow in size and lead to fracture (at load amplitudes much smaller than the critical static load) if cycled long enough.

It is convenient to envision three phases in the life of a fatigue crack: (i) initiation or nucleation, (ii) stable crack growth (otherwise known as the secondary stage) and (iii) fast (accelerated) growth leading to fracture. The total fatigue life of a structure can be written as the sum,

$$
N_{T}=N_{I}+N_{P}
$$

where $N_{I}$ is the crack initiation life, and $N_{P}$ is the crack propagation life which includes the stable as well as the accelerated stages of fatigue crack growth. The duration of the accelerated stage is usually negligible compared to that of the secondary stage. A series of four papers by the ASCE Committee on Fatigue and Fracture Reliability (Committee, $1982 \mathrm{a}-\mathrm{d}$ ) provides a comprehensive review of the subject of fatigue crack nucleation, growth and reliability.

The initiation phase of fatigue life in a virgin material has often been assumed (e.g., Murtaza and Akid, 1995) to constitute the growth of short cracks up to the size $a_{s}$, which is the transition length of short cracks into long cracks. As the short crack approaches $\boldsymbol{a}_{\boldsymbol{s}}$ in length, its behaviour and growth rate also approach those of long cracks (Kaynak et al, 1996). Once a long crack develops, fatigue damage growth enters the more familiar and widely documented domain of stable crack growth described by fracture mechanics.

Since fatigue is intimately connected with load cycling, it is often sub-divided into two. domains: high-cycle fatigue (with fatigue life, say, above a million cycles) and low-cycle: fatigue (with fatigue life below, say, $10^{5}$ cycles). In the former, the material initially is essentially defect-free, the local stresses are low and yielding is absent, and a substantial. portion of the fatigue life is spent in crack initiation. In the latter, the material could have initial defects (like a notch) and the loading is usually in the inelastic range.

Analogous to monotonic stress-strain curves, it is also possible to plot cyclic stressstrain curves for a material under a given fatigue loading condition. Two idealized fatigue loading conditions can be envisioned: strain controlled and stress controlled. In the former, cycling occurs such that the strain alternates between two fixed limits, whereas in. the latter, the applied stress alternates between two fixed limits. In either case, the shape and size of the hysteresis loops do not remain constant from the beginning of load cycling: to failure. The material either softens or hardens cyclically over the first few cycles, and 
then stabilizes in its stress-strain response giving rise to closed hysteresis loops (e.g., Endo and Morrow, 1969). Cyclic softening is said to occur if the stress range drops in successive loops under a given strain range (for strain-controlled cycling) or if the strain range increases in successive loops for a given stress range (under stress-controlled cycling). The exact opposite happens in cycling hardening. Finally, if cycled long enough, the loop tips start to move away from the equilibrium position and fracture occurs. The tips of the stable hysteresis loops from fully-reversed tests conducted at different load levels are plotted to obtain the cyclic stress-strain curve. Its equation can be provided in a format similar to that of the monotonic Ramberg-Osgood stress-strain relation (eq 2.2):

$$
\Delta \epsilon=\frac{\Delta \sigma}{E}+2\left(\frac{\Delta \sigma}{2 H}\right)^{M^{\prime}}
$$

where $\Delta \epsilon$ and $\Delta \sigma$ are the strain and stress ranges respectively, $E$ is the elasticity modulus, $H$ is the cyclic hardening modulus and $M^{\prime}$ is the cyclic hardening exponent. The first term on the right hand side of eq (2.9) gives the elastic strain range, while the second term gives the plastic strain range.

Alternating stresses are characterized by their maximum and minimum limits $\sigma_{\max }$ and $\sigma_{\min }$. Associated quantities like the mean stress $\sigma_{m}$ (the average), the stress amplitude $\sigma_{a}$ (the semi-difference), the stress ratio $R=\sigma_{\min } / \sigma_{\max }$, and amplitude ratio $A=\sigma_{a} / \sigma_{m}$ are used in several empirical fatigue laws. Similar quantities are defined and used for strain controlled fatigue.

The classical method of expressing the fatigue life of a member (in number of cycles, $\mathrm{N}$ ) is through an $\mathrm{S}-\mathrm{N}$ curve, in which $\mathrm{S}$ is usually the applied stress amplitude. The Basquin model is

$$
N S^{m}=C
$$

where $m, C$ are experimentally determined constants. For many materials (like carbon and alloy steels and some stainless steels) the S-N curve for fully reversed loading of a smooth polished specimen becomes flat at the endurance limit, $S_{e}$, below which fatigue failure does not occur. $S_{e}$ is commonly around $40-50 \%$ of the ultimate stress, $\sigma_{u}$, for low and intermediate strength steels and around $50 \%$ of $\sigma_{u}$ for wrought aluminum alloys (Dowling, 1993).

Instead of a stress-based approach to fatigue life (eq 2.10), a strain-based approach could be adopted. The Coffin-Manson model relates the total strain amplitude, $\epsilon_{a}$, to the fatigue life, $N$, as:

$$
\epsilon_{a}=\frac{\sigma_{f}^{\prime}}{E}(2 N)^{b}+\epsilon_{f}^{\prime}(2 N)^{c}
$$

where $E$ is the elastic modulus and the other four terms are empirical constants. Analogous to the cyclic stress-strain equation, eq (2.11) separates the total strain amplitude into its elastic and plastic components. The first term, which plots with a very flat 
slope on a log-log plot, represents the elastic strain amplitude, $\epsilon_{e a}$, and is negligible in the high-strain low-cycle region. On the other hand, the second term, representing the plastic strain amplitude, $\epsilon_{p a}$, has a much steeper slope and is negligible in the low-strain high-cycle region. The two plots intersect at the transition life, $N_{t}$ (Dowling, 1993). Therefore, the $\epsilon_{e a}-N$ relation, which dominates the strain-life relation in the high-cycle region, may be said to approximate the crack initiation life, $N_{I}$, while the $\epsilon_{p a}-N$ relation which dominates the strain-life relation in the low-cycle region, may be said to approximate the crack propagation life, $N_{P}$.

In the above discussion, the applied stresses (or strains) were implicitly assumed to cycle between two fixed limits during fatigue loading. In practical situations, however, stresses (or strains) seldom alternate between constant limits; instead variable amplitude loading is common (Committee, 1982c). Cycle counting in such cases can be done by the "rainflow method" (Dowling,1993). Miner's rule is often applied to assess the cumulative fatigue damage under variable amplitude loading:

$$
\begin{aligned}
D & =\sum_{i=1}^{N_{B}} \frac{n_{i}\left(S_{i}\right)}{N_{i}\left(S_{i}\right)} \\
& =1 \text { at failure }
\end{aligned}
$$

where damage, $D$, is measured in linear increments; $n_{i}$ and $N_{i}$ denote, respectively, the number of applied cycles and the number of cycles to failure at stress level $S_{i}$; and $N_{B}$ denotes the number of different stress-levels applied. Miner's rule does not take into account the order of the loading cycles, and can lead to serious under-prediction or overprediction of fatigue lives depending on the order of occurrence of the different stress levels (Schutz, 1993; Dowling, 1993). In general, it is observed in variable amplitucle loading that, a few cycles $\left(n_{1}\right)$ at a high stress level $\left(S_{1}\right)$ followed by cycling at a lower level $\left(S_{2}, n_{2}\right)$ causes greater damage than when the lower stress level $\left(S_{2}, n_{2}\right)$ is applied first (followed by $S_{1}$ for $n_{1}$ cycles).

The growth rate of long fatigue cracks along with the condition of their non-propagation has been successfully described (in the secondary or stable crack growth stage) by the Paris-Erdogan law

$$
\frac{d a}{d N}=C(\Delta K)^{m} ; \quad \Delta K>\Delta K_{t h}
$$

where $\Delta K=K_{\max }-K_{\min }$ is the range of the fluctuating stress intensity factor and $C, m$ are experimentally determined constants (unrelated to those in eq. 2.10). It has been widely observed (Barsom and Rolfe, 1987) that fatigue crack growth rate is zero below a threshold stress intensity factor range, $\Delta K_{t h}$, which strongly depends on the stress ratio $R$. The threshold range $\Delta K_{t h}$ therefore can be used to define the boundary between the initiation stage and the stable stage of fatigue crack growth. The boundary between the stable and accelerated crack growth stages may likewise be defined with the help of the mode I fracture toughness, $K_{I_{c}}$. Improvements in the Paris-Erdogan law have been sought by incorporating parameters like $R, \Delta K_{t h}$ and $K_{\mathrm{c}}$ into the original 
equation (Dowling, 1993). However, the Paris-Erdogan law has remained the most common empirical model of stable macroscopic fatigue crack growth under the assumption of linear elastic behavior. The length of the propagation life, $N_{P}$ (eq 2.8), in an initially undamaged specimen can be obtained by integrating the Paris-Erdogan law between the crack length limits $a_{s}$ and $a_{c}$, where $a_{s}$ is the threshold length of crack initiation and $a_{c}$ is the critical crack length at the onset of unstable crack growth. While using the Paris-Erdogan law to determine $N$ for other crack increments, it should be ensured that the limits of integration lie within the range $\left[a_{s}, a_{c}\right]$.

LEFM-based crack growth concepts break down at small or short crack sizes (e.g., Klesnil et al, 1984). Short cracks continue to grow at stress-intensities below the long crack threshold $\Delta K_{t h}$ described above. Moreover, short cracks may grow at rates higher than those for long cracks depending on the stress ratio (Kaynak and Ankara, 1992). Ignoring the short crack growth stage, or using long crack growth rate parameters (e.g., $m$ and $C$ in Paris-Erdogan law) for short crack growth "can lead to potential dangerous over-prediction of (fatigue) life" (Kaynak et al, 1996). The threshold crack length, $a_{s}$, below which LEFM (and consequently the Paris-Erdogan law) is not valid, may be approximately estimated (Dowling, 1993) with the help of two macroscopic quantities $S_{e}$ and $\Delta K_{t h}$ (both for $R=-1$ ):

$$
a_{s}=\frac{1}{\pi}\left(\frac{\Delta K_{t h}}{\Delta S_{e}}\right)^{2}
$$

It should be mentioned here that the fatigue crack initiation length, $a_{s}$, lacks a universally accepted definition (Martin and Wirsching, 1991; Min et al, 1996). Kujawski and Ellyin's comment (1992) highlights this: "Usually the crack initiation stage is associated with an arbitrary specified crack length. The crack length ranging from grain diameter to about $50-100 \mu \mathrm{m}$ is used, depending on the material and physical scale of interest." However, Martin and Wirsching (1991) used a value of $a_{s}=0.5 \mathrm{~mm}$ for structural welds and Kaynak et al (1996) chose a value of $1 \mathrm{~mm}$ for En7A steel. Murtaza and Akid (1995), on the other hand, chose $a_{s}=120 \mu \mathrm{m}$ for BS250A53 steel. Majumdar et al (1993) recommended a value of $0.002 i n(51 \mu \mathrm{m})$ as the initiation crack length in carbon steel.

\subsubsection{Randomness in Fatigue Crack Growth}

Fatigue crack growth is a random process. Test data in fatigue studies exhibit a relatively large amount of scatter in spite of tightly controlled test variables and conditions. This scatter is due to the intrinsic microstructural variations in the specimen. Weibull was among the first to put probabilistic fracture mechanics on a sound analytical basis.

To incorporate the inevitable scatter in test data, $\mathrm{S}-\mathrm{N}$ curves are sometimes drawn as a family of P-S-N curves (Provan, 1987), where $\mathrm{P}$ is the probability of failure at $\mathrm{N}$ cycles at constant amplitude stress, S. Log-normal and Weibull distributions have been widely used to model fatigue lives of structures (Committee, 1982a).

In applying the Paris-Erdogan law in fatigue reliability analysis, some researchers 
have assumed either $m$ or $C$ to be the random variable or the random process (the other being deterministic), and some have taken them to be jointly distributed (e.g., Ortiz and Kiremidijian, 1986). Rocha et al (1993) found $m$ and $\log C$ to be linearly dependent for high tensile steel. Thara and Misawa (1991) assumed $\mathrm{C}$ to be a non-stationary Gaussian process. Lawrence et al (1990) integrated the Paris law assuming that the initial and final crack sizes, component geometry, crack growth parameters and loading were random.

Deterministic growth rate models like eq (2.14) have been rendered stochastic by multiplying the right hand side with a non-negative random process in time (Lin and Yang, 1983; Spencer and Tang, 1988) as in the following:

$$
\frac{d A(t)}{d t}=C(\Delta K)^{m} X(t)
$$

where $A(t)$ is the random crack size and $X(t)$ is the random process. Alternately, arguing that uncertainty in crack growth rate arises out of inhomogeneity and randomness of material properties at or near the crack-tip, others (e.g., Ditlevsen, 1986; Ortiz and Kiremedjian, 1986; Dolinski, 1992) have introduced a multiplicative random function of the crack-tip position, $a$, rather than of $t$, in their stochastic models:

$$
\frac{d A}{d n}=C(\Delta K)^{m} X(a)
$$

Fatigue crack growth has often been idealized as a Markov process. The various approaches taken by different authors in this regard are summarized in the following paragraph.

Lin and Yang (1983) adopted a diffusive Markov process to obtain the first passage time to reach the critical crack size. In doing so, they acknowledged the fact that the instantaneous growth rate has a finite probability of becoming negative on account of the additive white noise, but they assured that "this error is negligible so long as the drift dominates the diffusion" with the caution that this condition "should be verified in each practical case". The drift and diffusion coefficients were obtained by the stochastic averaging method. Upon assuming a power law for crack growth and the Weibull distribution for the time to failure, the reliability at a given time was estimated. Spencer and Tang (1988) attempted to restrict the growth rate to non-negative values by multiplying the deterministic model with the non-negative process $10^{Z(t)}$, where $Z(t)$ was an exponentially correlated stationary Gaussian process, and obtained statistical moments of the time to failure using a finite element formulation. In an accompanying numerical example, however, no rational method to estimate the correlation length of $Z(t)$ was provided. Markov approaches to fatigue crack growth have also been used by Oswald and Schueller (1984), Bogdanoff and Kozin (1985), Lassen (1991), Zhao (1993), and Ishikawa et al (1993).

Detailed data on fatigue characteristics of different kinds of steel may be obtained from Barsom and Rolfe (1987). Experimental data from Virkler et al (1979) on fatigue crack propagation in 2024- T3 Al alloy have been used by many authors to verify 
their random crack growth models. Virkler et al (1979) performed constant amplitude (load-controlled, room-temperature) tests on 68 pre-cracked specimens and recorded the number of cycles to attain predetermined crack increments. They observed sudden increases and decreases in crack growth rates as if "the crack was passing through a different material possessing different properties" and commented that fatigue crack propagation "is not a stable, smooth, well ordered process."

\subsection{Corrosion}

\subsubsection{Overview and Types of Corrosion}

Corrosion is an electrochemical process in which a metal combines with other substances present in its environment under favorable conditions and forms various compounds. It therefore requires an electrolyte in contact with the metal, which could be moist air, an aqueous solution or some liquid other than water. Non-electrochemical reactions in which there is loss or alteration of material also are included in the general terminology of corrosion. Physical factors which govern corrosion and its rate include temperature, residual stress and cyclic loading rate. Corrosion-causing environments may be broadly classified as moist (e.g., water, aqueous solutions, moist air and moist soil) and dry (e.g., air, gases, steam at high temperature, molten metals).

Corrosion is a combination of oxidation-reduction and acid-base reactions. When iron corrodes, metallic iron is oxidized by $\mathrm{H}^{+}, \mathrm{H}_{2} \mathrm{O}$ or $\mathrm{O}_{2}$ into ferrous or ferric ions which in turn produce aquo- and/or aniono-complexes of iron (Sato, 1987). An example of a cell reaction where ferrous hydroxide is formed is (Clifton and Knab, 1989):

$$
\mathrm{Fe}+\frac{1}{2} \mathrm{O}_{2}+\mathrm{H}_{2} \mathrm{O} \rightarrow \mathrm{Fe}(\mathrm{OH})_{2}
$$

At relative humidity above $80 \%$, the adsorbed moisture film formed on iron has properties of liquid water and is able to hydrolyze metal ions as well as dissolve pollutants like $\mathrm{SO}_{2}$ and $\mathrm{CO}_{2}$ from the atmosphere (Reinhard et al, 1992).

Many metals can form a protective oxide layer when exposed to oxygen (atmospheric or dissolved in water). In an aqueous environment, different salts of weak acids also can form such passive layers on ferrous materials, under certain conditions of anion concentrations and pH of the solution (Reinhard et al, 1992). As long as this layer is not ruptured and remains intact over the surface, corrosion cannot initiate on the metal. However, mechanical actions (like abrasion, tension, fatigue crack formation), elevated temperature or chemical agents (like chlorides) can rupture or destroy this passive layer and initiate corrosion. If the restoration of the protective layer is prevented or is slower than the rate of attack, corrosion progresses.

Carbon steel lacks the ability to form the protective layer unless it is in a dry, clean atmosphere (Schweitzer, 1988). Thus it is particularly susceptible to corrosion in harsh 
but routinely encountered service conditions. Yet, due to its wide availability and relatively low cost, carbon steel accounted for approximately $88 \%$ of the tonnage delivered in 1984-85 for such diverse applications as marine structures, nuclear and fossil fuel power plants, bridges and other transportation structures, and chemical, mining and metal. processing industries (Craig, 1989).

The important forms of corrosion are described in brief in what follows (Berger, 1983). Following that, mechanistic and statistical models for uniform and pitting corrosion will. be presented in detail. Electrochemical models of corrosion are beyond the scope of this research.

Uniform corrosion is among the most common forms of corrosion that are encountered. It is characterized by a progressive and uniform thinning of an entire section. This kind of degradation is often addressed by providing an extra thickness called "corrosion allowance" while designing.

Pitting is by many accounts the most devastating form of corrosion (e.g., Provan and Rodriguez, 1989). This is a highly localized phenomenon, and is often not accompanied. by general loss of material. Moreover, the pits can be hidden under a surface of corrosion products, making their detection very difficult. Pits are initiated due to local acidity, inhomogeneity in the material, cracking etc. Hydrogen is believed to have a role in. initiating pits in the passive film on pure iron (Pyun et al, 1992). In many situations the maximum pit depth is of critical interest, rather than the distribution of pit depths over a given surface. This is particularly relevant when a single through-the-thickness crack is enough to cause failure, for example, due to leakage in a pipeline or loss of pressure boundary in a pressure vessel.

Stress corrosion cracking (SCC) is a result of corrosion caused or accelerated by external or internal stresses in the metal. Cyclic loading in a material can cause rupture of the passive layer, forming anodic areas at the points of rupture. This gives rise to corrosion fatigue. Fretting is the small relative movement (of the order of 100 microns) between two adjoining metal pieces. This mechanical action can promote an oxidation reaction of the metal (Waterhouse, 1972) by removing the protective oxide layer or by increasing the reactivity of the metal due to plastic deformation.

Other forms of corrosion include galvanic corrosion, erosion corrosion, cavitation corrosion, intergranular corrosion and biological corrosion.

\subsubsection{Mechanistic and Statistical Models of Corrosion}

According to Komp (1987), most atmospheric corrosion data follow a power function of time, $t$ :

$$
C(t)=A t^{B}
$$

NUREG/CR-6546 
where $\mathrm{C}$ is the average loss of section, and $A, B$ are constants. Similarly, by analyzing experimental data and fitting a least-square curve, Mabuchi et al (1991) found the weight of corroded iron in high-temperature water to be related to exposure time by

$$
W(t)=a t^{b}
$$

where $a$ and $b$ are constants. The corrosion rate appears generally to increase with temperature and dissolved oxygen in water.

The time required for corrosion to initiate is a random variable. This random initiation time $\tau_{0}$ (prior to which corrosion penetration is zero) can be introduced to the model in eq (2.18) as (Ellingwood and Mori, 1993):

$$
C(t)=A\left(t-\tau_{0}\right)^{B}, \text { for } t \geq \tau_{0}
$$

Failure to include this initiation time leads to a conservative estimate of remaining service life or residual strength of a structure.

Power laws of the above form have also been adopted for pitting corrosion depth. According to Joshi (1994), the rate of pitting corrosion can be $3 \times 10^{5}$ to $1 \times 10^{6}$ times more rapid than general corrosion in the same component subject to the same environment. Strutt et al (1985) observe that "many corrosion profiles do not fit any of the commonly used statistical models, particularly when a combination of corrosion processes operate at the same time, giving multimodal distributions." However, they suggest that the maximum depth has a type I distribution. Markov models of pitting corrosion have been developed by Mola et al (1990), Provan and Rodriguez (1989) and Williams et al (1985). Stochastic models of comparable sophistication are not available for general corrosion.

\subsection{Appraisal of Existing Methods}

The existing methods summarized above for estimating accumulated damage or for predicting the time to failure are mostly empirical in nature. Purely empirical methods (like the $\mathrm{S}-\mathrm{N}$ curve for fatigue life) lack the ability to provide information on the residual strength of a structure as a function of time, the knowledge of which is essential in evaluating the safety of a degrading structure. Some of the methods also require an estimate of the time to failure of an existing structure in order to find out the remaining service-life (e.g., Miner's rule); this information may not be available, particularly for non-generic structures.

In this section, the shortcomings of the available methods in assessing damage and residual life or strength of deteriorating structures are discussed. Sections 2.5.1 and 2.5.2 address the models discussed above on fatigue, creep and corrosion. Section 2.5.3 examines several state-variable type "damage variables" - non-dimensional quantities 
taking values between 0 and 1 - that do not have mechanistic bases (unlike $\mathrm{CDM}^{8}$ ) but have been proposed in the literature to quantify structural deterioration. This will help set the CDM-based damage variable, introduced next in Chapter 3, apart from its look-alikes and will underline the advantage of a CDM-based formulation in determining the residual strength of a damaged structure.

\subsubsection{Fatigue, Fracture and Creep}

At constant amplitude stress or strain, the $S-N$ approach for fatigue life prediction provides information on the number of cycles to failure, $N_{f}$ (failure is defined either as fracture or as a pre-determined drop in the peak stress), at a given stress amplitude and stress ratio. This empirical approach is, however, not able to offer any estimate of the remaining strength of a member (e.g., ultimate strength or plastic moment capacity) that has passed through a certain fraction of $N_{f}$. Consequently, an S-N curve may not be useful in many instances of reliability analysis of a fatigued structure.

Miner's rule provides an empirical method to predict service life-time in metal fatigue under variable amplitude loading. One known shortcoming of this method is the observed fact that fatigue damage does not always grow linearly with number of applied cycles; rather, the major portion of service-life may be spent without any manifestation of reduced capacity, and damage becomes apparent and grows visibly at an increasing rate only towards the end of the life-time (e.g., Paas et al, 1993; Pasic, 1992; Tiejun and Zhiwen, 1990). A second difficulty in adopting a Miner's rule type of damage assessment (due to its a posteriori nature) is in evaluating the remaining service life of an existing structure where the total service-life is still unknown. A third objection to using Miner's rule occurs when the cycling takes place with varying amplitudes. In such a situation Miner's rule predicts the same value of accumulated damage irrespective of the order of appearance of the different amplitudes, which is contrary to experimental observations (e.g., Schutz, 1993; Kutt and Bieniek, 1988; Wheeler, 1972). Finally, the residucul strength of a structure in service cannot be predicted by a method that uses Miner's rule to assess damage. These deficiencies point to the need for more rational fatigue damage growth models in place of the phenomenological ones.

No conceptual breakthrough has yet been achieved in predicting the time to initiation of cracks in a virgin material under fatigue loading. However, two approaches of predicting $N_{I}$ based on macroscopic crack concepts are in existence: (i) In an S-N type approach, the number of cycles correspond to a specified threshold crack length (usually less than $0.5 \mathrm{~mm}$ ) under a given load amplitude. However, no unique definition of the threshold crack length exists in the fracture mechanics community (Kujawski and Ellyin, 1992) and this approach, like its macroscopic counterpart, is unable to provide any estimate of the residual strength at the end of the crack initiation period. (ii) Attempts also

\footnotetext{
${ }^{8}$ Continuum Damage Mechanics (CDM) relates damage to the microstructural deterioration in a structure ( $\sec 3.2)$.
}

NUREG/CR-6546 
have been made to come up with crack growth laws analogous to the Paris-Erdogan law for short cracks with different $C$ and $m$ values from those for long cracks (eq 2.14), for the same material and loading condition (e.g., Murtaza and Akid, 1995). Theoretically, this would enable one to estimate $N_{I}$ (eq 2.8) by integrating the short crack growth rate between the limits $a_{0}$ (short crack initiation length) and $a_{s}$ (long crack initiation length, eq 2.15) once the parameters ( $C$ and $m$ for the short crack growth law) are known. However, not only is it more difficult to obtain the $C$ and $m$ parameters by recording data on the growth of short cracks (which are by definition microscopic in size), but this Paris Law-type approach is extremely sensitive to the microscopic initial crack length $a_{0}$ (as illustrated in Kaynak et al, 1996). Neither any standard definition nor any reliable method of measurement is known to exist for the initial length $a_{0}$ which is supposed to be only as large as the grain size $(\sim 10-100 \mu \mathrm{m})$. Another difficulty in using a ParisErdogan type law for short cracks is their presence in abundant numbers in a specimen: It is not possible to predict which short crack in a virgin material subjected to cyclic loading would finally give rise to a long crack signalling the end of the crack initiation phase. In fact, this is just another empirical approach that does not deal with short crack growth from a mechanistic basis, but instead uses a curve fitting technique on the available data.

Hence, a totally new approach for predicting the fatigue crack initiation time in a virgin material is required - one that is completely independent of threshold crack sizes and empirical growth parameters for microscopic cracks. It will be shown in Chapter 4 that a CDM-based fatigue damage growth law provides estimates of $N_{I}$ in terms of macroscopically obtained material parameters.

A linear incremental damage growth rule exists for creep which was proposed by Robinson (1952). According to this model, the creep damage caused by load $S_{i}$ applied for time $t_{i}$ at a particular temperature is $D_{i}=t_{i} / T_{i}$, where $T_{i}$ is the rupture time at the same load and temperature. This model has all of the limitations of the fatigue models to which it is similar and experimental evidences of non-linear creep damage growth and load sequencing effects are available in the literature (e.g., Abo El Ata, 1972).

\subsubsection{Corrosion}

In his 1990 paper, Rogers laments "it is surprising that in the literature of corrosion failure prediction there are very few instances where statistical methods were applied." Yet, the evidence is compelling that a significant amount of randomness is involved in corrosion initiation and growth in steel structures (Ellingwood et al, 1996). All existing methods to describe growth of corrosion use empirical laws which have obvious shortcomings as stated at the beginning of this section. Nevertheless, these empirical laws can be applied to model the effect of corrosion on section geometry, and the residual strength as a function of corrosion loss may be obtained under appropriate limit states. However, there is also some evidence that corrosion may significantly alter the ductility of steels 
(Cherry, 1995), and this phenomenon is not addressed by existing empirical laws.

\subsubsection{The "Damage" State Variable}

The mechanics and thermodynamics-based CDM damage state variable will be introduced in the next chapter, and damage growth laws due to creep, fatigue and plastic deformation will be derived subsequently. However, there exist in the literature several other methods which also define and quantify structural "damage" in terms of state variables. These methods more often have phenomenological rather than mechanistic bases. Before moving on to the CDM-based models of damage growth, some of the other (phenomenological) definitions of structural damage are briefly examined in this subsection, which will provide a contrast to the mechanics-based formulation developed in the subsequent chapters of this report.

The Miner's law in fatigue (and the analogous one in creep), which in fact takes a state variable approach by expressing damage as the elapsed time normalized by failure time $\left(D=n / N_{f}\right.$ or $\left.t / T_{f}\right)$, has already been discussed in detail in sec 2.5.1. The following comment by Hult (1987) made in his review of continuum damage mechanics is pertinent here: "the use of the [Miner's] failure condition must be preceded by testing at all constant load levels $\left[S_{i}\right] \ldots$ In contrast to this, the CDM damage concept is related only to the current mechanical state of the material. Detailed knowledge about the conditions for deterioration of the material structure would make it possible to predict the load carrying capacity or lifetime without first performing extensive testing."

The change in the dynamic response of a structure has been used in the literature to quantify damage. For example, the increase in the squared natural period (normalized by the square of the damaged natural period) of a structure has been proposed as a measure of the accumulated damage (DiPasquale and Cakmak, 1989). Similar approaches have also been taken by Agbabian et al (1991) and Hearn and Testa (1991).

Diao (1995) has equated the damage variable $D$ with the failure probability, $F(t)$ (the cumulative probability distribution of the time to failure at $t$ ), arguing that it is a scalar quantity taking values between 0 and 1 and that it adequately represents the randomness in the material microstructure.

Claiming that fatigue damage is influenced by the "equivalent crack length" as well as the surface crack density which in turn is related to the plastic strain range, $\Delta \epsilon$, Pasic (1992) defined fatigue damage as

$$
D=b a^{c}(\Delta \epsilon)^{n}
$$

where $b, c, n$ are material parameters and $a$ is the normalized crack length.

Krajcinovic and Silva (1982) and Breysse (1990) idealized a volume of material as consisting of a large number of microscopic elastic springs of identical stiffness (some of which fracture under strain causing damage to the material), with equal load sharing 
among the unbroken springs. They defined damage as the ratio of the fractured to the total number of springs in the material volume.

Acoustic Emission (a non-destructive evaluation method that uses sound emitted from a strained structure to estimate the state of its damage) has been applied to estimate stress-controlled fatigue damage of Incoloy specimens (Fang and Berkovits, 1995). Denoting the intensity of AE occurrence by $\eta$ and the intensity at failure by $\eta_{f}$, they defined the damage variable $D=\eta / \eta_{f}$. However, no attempt was made to establish this quantity $D$ on a mechanistic basis or to relate it to material properties like stiffness. Plots of $D$ with normalized cycles $n / N_{f}$ were found to accelerate as their values approached 1 .

In contrast to the above approaches, continuum damage mechanics (CDM) defines damage in a rational way that is rooted in mechanics. As will be shown in Chapter 4 , it is extremely well suited to deal with the accumulation of damage that arises out of overall microstructural deterioration, rather than the growth of a dominant flaw. It makes intuitive sense to describe damage growth as an irreversible process and, indeed, CDM has been clearly shown to follow the thermodynamics of irreversible processes (e.g., Hansen and Schreyer, 1994). However, the existing CDM-based damage growth models, as will be explained in sec 3.3, lack continuity with the first principles of thermodynamics and often contain undetermined material constants. Moreover, the inherent probabilistic nature of damage accumulation has yet to be taken into account in the existing formulations. These shortcomings have been been addressed in the present research. 


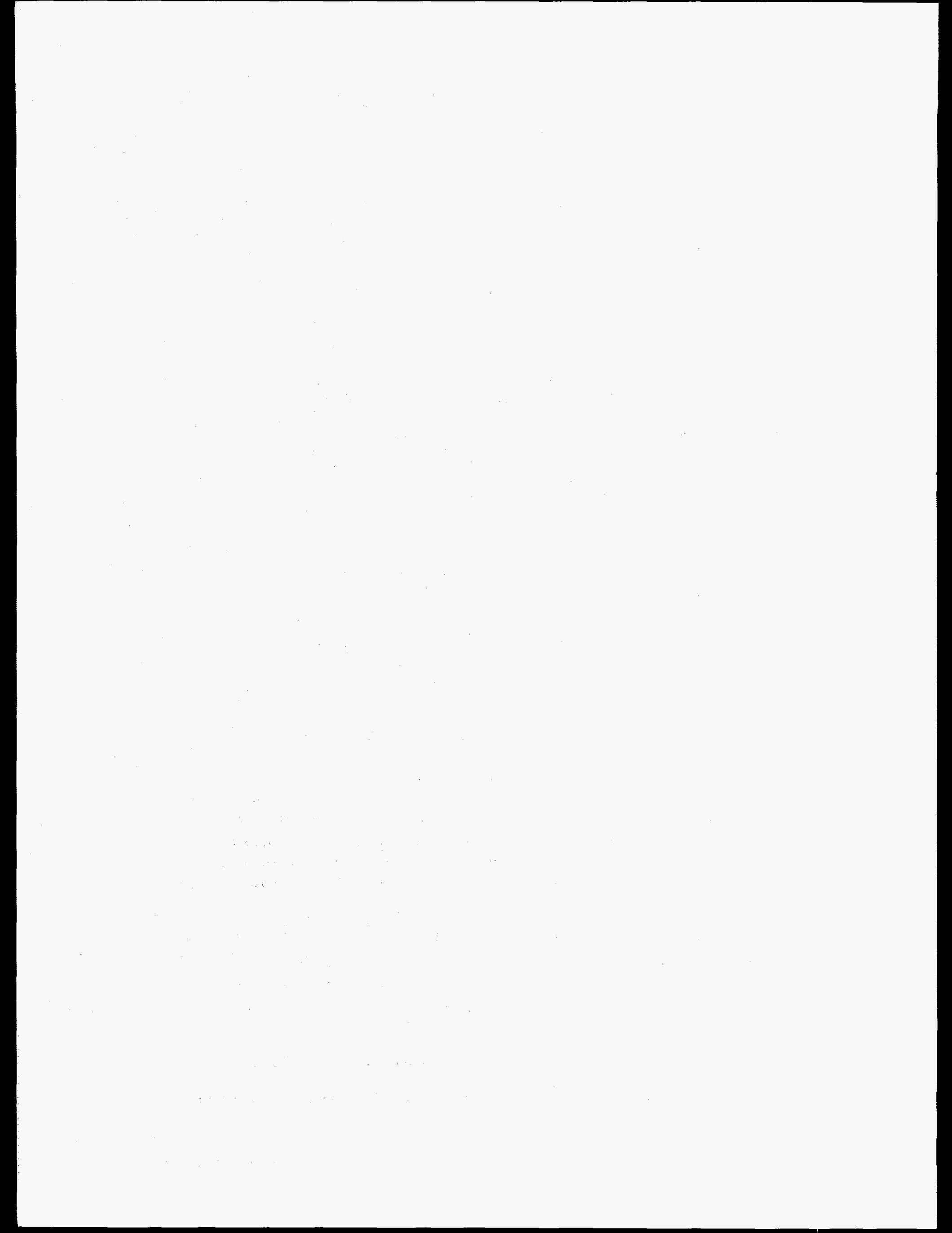




\section{Chapter 3}

\section{CDM-based Approaches to Modeling Structural Deterioration}

\subsection{Introduction}

Continuum damage mechanics (CDM) is a relatively new development in solid mechanics and concerns the distribution, characterization and growth of microstructural defects with the help of macroscopic state variables (Krajcinovic, 1984; Simo and Ju, 1987; Hult, 1987). Damage accumulation is considered to be a dissipative process that obeys the laws of thermodynamics (Hansen and Schreyer, 1994). Physically, the CDM damage concept represents a loss of material integrity which reduces the capacity of a damaged component to bear applied stresses. Such loss of material integrity is caused by various processes at the microscopic level (Woo and $\mathrm{Li}, 1993 \mathrm{a}$ ), and continuum damage mechanics seeks to express the effects of those microscopic processes by quantities that can be measured at the macroscopic level. Examples of damage-causing mechanisms include ductile deformation, fatigue, creep, embrittlement and stress corrosion. Damage growth equations for one or a combination of these processes may be derived in the context of CDM.

It will be shown in this and the subsequent chapters that a CDM-based approach to structural damage avoids much of the empiricism that is inherent in many of the existing methods ${ }^{1}$. Unlike most empirical methods, CDM-based damage growth models can provide estimates of residual strength which may be used to determine the safety of a degrading structure.

The present chapter begins by introducing fundamental concepts of continuum dam-

\footnotetext{
${ }^{1}$ A critical appraisal of the existing methods of modeling structural deterioration was presented in $\sec 2.5$
} 
age mechanics. Following that, a review of the existing CDM-based models and their shortcomings are presented. The development of the proposed method is then taken up starting from the first principles of thermodynamics. Variational results leading to a general set of partial differential equations describing damage growth in deformable bodies are established at the end of this chapter. These equations are specialized in Chapter 4 and are applied individually to ductile, creep and fatigue damage. The proposed method is extended into the area of stochastic damage growth in Chapter 5.

\subsection{Fundamentals of Damage Mechanics}

\section{Definition of damage}

In CDM, the damage variable, $D(\hat{n})$, on an elemental cross-sectional plane (with unit normal $\hat{n}$ ) is quantified by the surface density of cracks and voids which are considered "lost" in regard to the cross-section's capacity to transmit applied stresses. However, $D(\hat{n})$ is not simply the net fractional area of the discontinuities at the given cross-section, but is weighted by the effects of stress concentration at the edges of discontinuities and the interaction among the defects (Lemaitre, 1985). If $A_{v}$ denotes the sum of the actual area of the individual defects present on an elemental cross-section of area $A_{0}$ and orientation $\hat{n}$ (fig. 3.1$)^{2}$, then the area effectively lost at this section, $A_{l}$, is larger than $A_{v}$ and damage on this cross-section is the ratio:

$$
D(\hat{n})=\frac{A_{l}}{A_{0}}>\frac{A_{v}}{A_{0}}
$$

The above definition of damage imparts to $D(\hat{n})$ a directional nature, and as a result, damage can be expressed by tensors of order one (vectors), two, four or even eight (Krajcinovic, 1984). If, however, the weighted fractional loss in area is the same in every orientation within the material, then damage is independent of $\hat{n}$ and is said to be isotropic. Isotropic damage is quantified by one single scalar variable, $D$, a dimensionless number between zero and one. A brief history of CDM from its inception may be found in Krajcinovic (1984) and Hult (1987).

\section{Effective Stress}

The concept of effective stress is used extensively in CDM (Chaboche, 1988; Kachanov, 1986; Lemaitre, 1985). It relates the stress distribution within a damaged material to the state of damage within the material. The effective stress in the direction $\hat{n}$ is defined as

$$
\tilde{\sigma}=\frac{\sigma}{1-D(\hat{n})}
$$

\footnotetext{
${ }^{2}$ Figures and Tables are placed at the end of each chapter.
} 
where $\sigma$ is the nominal stress. Generalizing, the effective stress tensor is

$$
\tilde{\sigma}=\mathbf{M}(\mathbf{D}): \sigma
$$

where $\sigma$ is the nominal Cauchy stress tensor and $\mathbf{M}(\mathbf{D})$ is called the damage effect tensor (Chow and Wei, 1991). When damage is isotropic, the effective stress reduces to

$$
\tilde{\sigma}=\frac{\sigma}{1-D}
$$

and the damage effect tensor reduces to

$$
\mathbf{M}=\frac{1}{1-D} \mathbf{I}
$$

in which $I$ is the identity matrix.

\section{Principle of Strain Equivalence}

The constitutive law for damaged materials is derived from the principle of strain equivalence. This principle states (Chaboche, 1988):

A damaged volume of material under the nominal stress $\sigma$ shows the same strain response as a comparable undamaged volume under the effective stress $\tilde{\sigma}$.

Applying the principle of strain equivalence to the elastic strain:

$$
\begin{aligned}
\sigma & =\tilde{\mathbf{C}} \epsilon_{e} \\
\tilde{\sigma} & =\mathbf{C} \epsilon_{e}
\end{aligned}
$$

where $\mathbf{C}$ and $\tilde{\mathbf{C}}$ are the undamaged and damaged elasticity matrices respectively. Using the definition of effective stress, the two can then be related by

$$
\tilde{\mathbf{C}}=\mathbf{M}^{-1} \mathbf{C}
$$

This leads to the familiar expression for the reduced modulus of elasticity $\tilde{E}$ under the assumption of isotropic damage (e.g., Lemaitre, 1984):

$$
\tilde{E}=E(1-D)
$$

where $E$ is the modulus of elasticity for the undamaged material and the implicit assumption is that the Poisson's ratio is unaffected by damage (deVree et al, 1995). In fact, the modulus of elasticity and the Poisson's ratio both are affected when damage is considered anisotropic (e.g., Chow and Wei, 1991).

Equation (3.9) provides a means to estimate the state of damage in a component by experimentally determining its reduced modulus of elasticity and normalizing it by 
the original stiffness or the stiffness of a comparable undamaged component. Fig 3.2 describes a laboratory procedure to measure the progressive damage in a ductile specimen by periodically unloading it and measuring the slope of the unloading curve. The damaged stiffness may also be measured in situ by one of several non-destructive meth.ods, for example, ultrasonic pulse velocity, electrical resistivity etc (Lemaitre, 1992). This suggests a scheme to continuously monitor the integrity of an in-service structure, perhaps in an automated manner, which could be used to alert the maintenance staff in case the stiffness decreases below a pre-determined safe value or the rate of decrease starts to accelerate.

\section{Critical Damage}

By definition, damage accumulation is an irreversible process, and the overall damage variable is a non-decreasing function of time provided that corrective human intervention does not occur. In CDM, failure occurs when the damage variable reaches the critical damage $D_{c} \leq 1$. In the context of CDM, "failure" is not necessarily fracture, but is the condition when an essential assumption in continuum damage mechanics, namely damage arising out of a volume-wide degradation of the material microstructure, ceases to be applicable. It is that point when the damage-causing process becomes localized and leads to the growth of a dominant defect. Chaboche (1988) described it as the "breaking up of the continuum volume element". This particular ability of damage mechanics to quantify damage that accumulates prior to the formation of a macroscopic defect makes CDM particularly suitable to model damage growth in the initial "defect-free" stage, unlike most of the existing empirical methods which need a measurable flaw to be useful $(\sec 2.5)$.

In the absence of the formation of a macroscopic defect, the state of rupture itself may be defined as failure. For ductile damage, $D_{c}$ is widely interpreted as the value of damage corresponding to rupture (e.g., Lemaitre, 1985), presumably because ductile fracture in monotonic loading occurs by necking and not by the nucleation and growth of a macrocrack. However, $D_{c}$ signals the initiation of a macroscopic crack in fatigue (e.g., Lemaitre, 1984; Pasic, 1992; Dhar et al, 1996, Chow and Wei, 1991) as well as in creep (e.g., Lemaitre, 1984).

The above interpretations of failure allow $D_{c}$ to have values less than unity, as opposed to many phenomenological models (like Miner's rule in fatigue and Robinson's rule in creep) which in effect require the critical damage to be identically equal to one in every loading situation. Fractographic analysis of ductile rupture (for example, by Le Roy et al (1981), who examined the area-fraction of voids ${ }^{3}$ in ruptured carbon steel specimens)

\footnotetext{
${ }^{3}$ As explained in sec. 3.2 the damage variable, $D$, is greater than the net fractional area of defects, $A_{v} / A_{0}$, on a cross-section because of stress-concentration at the edges of the discontinuities and void interactions. Indeed, Le Roy et al (1981) reported $A_{v} / A_{0}$ values of around 4 to $7 \%$ at failure which are almost an order of magnitude lower than $D_{c}$ values (around $20 \%$ to $30 \%$ ) reported for carbon steels elsewhere (e.g., Lemaitre, 1984; Lemaitre, 1992; Tie-Jun, 1992).
} 
has corroborated the fact that $D_{c}$ is not always equal to 1 . Indeed, $D_{c}$ is often believed to be an intrinsic material property (e.g., Chow and Wei, 1991). This suggests that once $D_{c}$ is determined from a particular experiment (e.g., a simple tension test) for a particular material at a given temperature, it could be used to predict failure in one or a combination of other degradation mechanisms (e.g., creep-fatigue interaction), providing a unified approach to modeling structural deterioration. Experimentally determined values of $D_{c}$ range anywhere between 0.15 and 0.85 depending on the material (e.g., Lemaitre, 1985).

The correlation between critical damage and failure however has not been maintained consistently. Lemaitre (1984) allowed values of $D_{c}$ anywhere between 0 and 1 for ductile and creep damages, but assumed $D_{c}=1$ for fatigue failure. Kachanov (1986) assumed $D_{c}=1$ irrespective of the damage-causing process ${ }^{4}$, though he recognized the possibility of values lower than 1 if "damage is localized and macrocracks occur."

\subsection{Review of Existing CDM-based Methods}

The existing CDM-based methods of describing damage growth can be broadly classified into one of the following two approaches: (i) Setting up phenomenological or "kinetic equations" of damage growth, and (ii) Postulating thermodynamic potential functions of dissipation due to damage growth. Either approach, as will be shown subsequently, introduces unknown material constants in the damage growth equations, and it is usually difficult to obtain numerical estimates of these material constants. The proliferation of undetermined material parameters in the existing CDM-based damage growth models has caused concern in the damage mechanics community (e.g., Krajcinovic and Mastilovic, 1995). This problem, coupled with the arbitrariness associated with the choice of the kinetic equations or dissipation potential functions (which do not descend directly from the first principles of mechanics or thermodynamics), poses an impediment to a wider acceptance of CDM in modeling structural deterioration.

\subsubsection{Kinetic Equation Method}

The general form of the available kinetic equations of damage growth is:

$$
\dot{D}=f(D, \mathbf{x} ; \omega)
$$

where $\mathbf{x}$ is the set of state variables (e.g., strain) and $\omega$ denotes a set of material parameters. Kachanov was among the first to introduce the concept of continuum damage mechanics. In his pioneering work, Kachanov (1958) proposed the following kinetic equation (applicable to brittle fracture and creep damage under uniaxial tension), in terms

\footnotetext{
${ }^{4}$ Kachanov actually introduced a "continuity variable", $\Psi=1-D$, and took $\Psi=0$ at failure.
} 
of the "continuity variable", $\Psi=1-D$ :

$$
\frac{d \Psi}{d t}=-A\left(\frac{\sigma}{\Psi}\right)^{n}
$$

where $A>0, n \geq 1$ are material constants, and the initial condition is $\Psi=1$ at $t=0$. For low-cycle fatigue, this is modified to (Kachanov, 1986):

$$
\frac{d \Psi}{d t}= \begin{cases}-A\left(\frac{\sigma}{\Psi}\right)^{n}, & \text { if } \sigma>0 \\ 0, & \text { if } \sigma \leq 0\end{cases}
$$

For high-cycle fatigue, Kachanov's kinetic equation of damage growth is:

$$
\frac{d \Psi}{d t}= \begin{cases}-\frac{B}{\sigma_{u}}\left[\sigma_{\max }^{b}-\sigma_{\min }^{b}\right], & \text { if } \sigma>\sigma_{f} \\ 0, & \text { if } \sigma \leq \sigma_{f}\end{cases}
$$

where $B, b$ are constants and $\sigma_{f}$ is the fatigue limit. A similar phenomenological expression for fatigue damage growth was used by Jun and Xing (1995).

Carmeliet and Hens (1994) assumed the following damage growth model for strainsoftening materials and applied it to direct-tension type loading:

$$
\dot{D}=C \frac{1}{\bar{\epsilon}^{2}} \dot{\bar{\epsilon}}, \quad \bar{\epsilon}>\epsilon_{0}, \dot{\bar{\epsilon}}>0
$$

where $\dot{\bar{\epsilon}}$ is the "average equivalent strain rate", $\mathrm{C}$ is a positive material constant and $\epsilon_{0}$ is a threshold value.

Paas et al (1993) proposed a damage growth law for brittle as well as fatigue damage as:

$$
\dot{D}=C \bar{\epsilon}^{d}\left(D_{c}-D\right)^{-e}
$$

where $C, d, e, D_{c}$ are material constants.

\subsubsection{Thermodynamic Potential Method}

The basic approach in this method can be summarized in two steps: Postulate a thermodynamic potential function, $\phi_{i}$, which describes the intrinsic dissipation rate per unit volume:

$$
\phi_{i}=\phi_{i}(Y, \dot{x} ; \omega)
$$

such that it contains the damage energy release rate, $Y$, explicitly; and differentiate $\phi_{i}$ with respect to $Y$ in order to obtain the damage growth rate:

$$
\dot{D}=\frac{\partial \phi_{i}}{\partial Y}
$$


Isotropic damage has been used in eqs (3.16) and (3.17) for simplicity. The rationale of the above approach is outlined in the following:

As mentioned earlier, damage growth is a dissipative process governed by the laws of thermodynamics ${ }^{5}$. In an isothermal process, the rate of intrinsic dissipation, $\phi_{i}$, can be expressed in terms of the "dissipation variables", $\mathbf{H}$, as (Hansen and Schreyer, 1994):

$$
\phi_{i} \equiv-\rho \frac{\partial \psi}{\partial \mathbf{H}} \dot{\mathbf{H}} \geq 0
$$

where $\psi$ is the free energy per unit mass and $\rho$ is the mass density. It should be noted that the assumption that the intrinsic dissipation rate is positive at every instant and at every point within a continuum has been severely criticized, e.g., by Woods (1981). If $\mathbf{D}$ denotes the partition of $\mathbf{H}$ that signifies the "damage" variables, then the "damage energy release rate", $\mathbf{Y}$, is defined as

$$
\mathbf{Y}=-\rho \frac{\partial \psi}{\partial \mathbf{D}}
$$

As a duality,

$$
\dot{\mathbf{D}}=\frac{\partial \phi_{i}}{\partial \mathbf{Y}}
$$

which in the case of isotropic damage becomes (cf eq 3.17)

$$
\dot{D}=\frac{\partial \phi_{i}}{\partial Y}
$$

Previous efforts from this point on have been directed to the essential step of proposing expressions for the dissipation rate in terms of $\mathbf{Y}$ (or $Y$ in case of isotropic damage), and then differentiating them with respect to $\mathbf{Y}$ (or $Y$ ), as the means of obtaining equations for $\dot{\mathbf{D}}$ (or $\dot{D}$ ) (e.g., Lemaitre 1985; Chow and Wei, 1991; Chaboche, 1988; Hansen and Schreyer, 1994; Tie-Jun, 1992, Woo and Li, 1992 etc). The method proposed in this report makes a departure from this as will be shown later.

The necessity of conceiving dissipation functions in order to obtain practical equations of damage growth, instead of deriving them directly from the first principles of thermodynamics and mechanics, is a handicap of the existing methods. "Realistic" and "physically consistent" expressions for the dissipation rate in terms of $\mathbf{Y}$ and other variables, unfortunately, have more to do with the necessity of producing manipulable expressions for $\dot{\mathbf{D}}$ than with the physics of damage accumulation. Apart from the difficulty in conceptualizing "the damage energy release rate", $Y$, and how it governs the dissipation, such methods of deriving damage growth equations are by nature subjective since different authors may propose different forms of the dissipation function for the same damage mechanism. This may prove to be an impediment to a wider acceptance of damage mechanics in predicting damage growth. As mentioned at the beginning of this section,

\footnotetext{
${ }^{5}$ The thermodynamics of damage is discussed in detail in section 3.4 in the context of the development of the present method.
} 
the prevalent practice of indiscriminately choosing internal variables, of justifying arbitrary continuum damage models on the lone merit that they satisfy the Clausius-Duhem inequality, and the proliferation of undetermined material parameters that are spawned by such models has been criticized by Krajcinovic and Mastilovic (1995).

To illustrate these difficulties, some of the dissipation functions published in the literature are discussed below:

Lemaitre (1985) assumed an expression for the dissipation rate for ductile plastic damage as

$$
\phi^{*}=\frac{S}{a+1}\left(\frac{-Y}{S}\right)^{a+1} \dot{\epsilon_{p}},
$$

$S$ and $a$ being material and temperature dependent constants. The damage evolution law for ductile plastic damage that arises out of eq (3.22) is

$$
\dot{D}=\left(\frac{K^{2}}{2 E S} T \epsilon_{p}^{\frac{2}{M}}\right)^{a} \dot{\epsilon_{p}}, \quad \epsilon_{p}>\epsilon_{0}
$$

where $K, M$ are Ramberg-Osgood parameters, $T$ is a "triaxiality factor" which reduces to 1 in case of uniaxial loading, $\epsilon_{p}$ is the plastic strain and $\epsilon_{0}$ is the threshold plastic strain of damage accumulation. Tie-Jun (1992) sought to improve the above model by introducing another unknown constant $\alpha$ and incorporating the critical plastic strain in it.

Chaboche (1988) used a dissipation rate for ductile plastic damage as:

$$
\phi^{*}=\tilde{\sigma}_{e q}-R-k+\frac{S}{2} \frac{1}{1-D}\left(-\frac{Y}{S}\right)^{2}
$$

where $\tilde{\sigma}_{e q}$ is the equivalent effective stress, $R$ and $k$ are associated with the Von Mises plastic potential and $S$ is a material parameter.

Chow and Wei (1991) proposed a dissipation rate for fatigue:

$$
\Phi_{d}=\left(\frac{1}{2} \mathbf{Y}^{\mathrm{T}}:[\mathrm{J}]: \mathbf{Y}\right)^{\frac{1}{2}}-\left(B_{0}+B(w)\right)
$$

where [J] is a symmetric $2 \times 2$ matrix; and $B_{0}$ and $B(w)$ are the initial and the incremental strain hardening threshold.

To highlight the limitations of damage growth laws arising out of the thermodynamic potential method, we choose the model proposed by Lemaitre (eq 3.23) as a representative case. This equation contains two unknown constants $a$ and $S$, which cannot be obtained from standard test results. Lemaitre eliminated the first constant by assuming that $M \sim \infty$, which however may not hold for all materials. The second constant, $S$, was eliminated by normalizing the equation by the boundary condition at rupture (i.e., $D=$ $D_{c}$ at $\left.\epsilon_{p}=\epsilon_{f}\right)$ :

$$
D=D_{c}\left(\frac{\epsilon_{p}-\epsilon_{0}}{\epsilon_{f}-\epsilon_{0}}\right) ; \epsilon_{p} \geq \epsilon_{0}, M \rightarrow \infty
$$


This however introduced the need to know the critical damage a priori, and consequently, this model can no longer independently predict the unknown value of $D_{c}$ given $\epsilon_{p}=\epsilon_{f}$. It clearly would be more desirable to have a model that predicts $D_{c}$ independently using readily available material data, which then could be applied to any failure mechanism of interest. Lemaitre (1992) later attempted to measure the constant $S$ experimentally, but admitted difficulty in getting an accurate estimate of the constant.

The remainder of this report will be directed towards the development of a new method of modeling structural deterioration from first principles and applying it to ductile, creep and fatigue damages. The motivation is to come up with rational damage growth laws with as little empiricism as possible, so that they do not require one to postulate potential functions or kinetic equations and, at the same time, do not introduce unknown material parameters in the formulation.

\subsection{Thermodynamic Foundations of Damage Growth}

The thermodynamic principles underlying damage accumulation have been successfully established in CDM (e.g., Hansen and Schreyer, 1994). However, the existing thermodynamics -based CDM models of damage growth lack continuity with the first principles of thermodynamics and mechanics, and contain unknown material constants, as was discussed in the previous section. The present method will be shown to make a departure from the existing approaches, and damage growth laws starting from the first principles of thermodynamics will be derived. We begin this section by introducing the notion of irreversible processes. An expression for the dissipation rate (under isothermal conditions) is then established in terms of the evolution of the Helmholtz free energy. Conditions in which the free energy may be considered stationary are discussed, and partial differential equations of damage growth in deformable bodies are obtained. In Chapter 4, assuming isotropic damage and uniaxial loading, these differential equations are solved for different constitutive models, and damage growth laws for ductile deformation, creep and fatigue are developed and compared with published experimental results.

\subsubsection{Thermodynamically Irreversible Processes}

A system is said to be in stable thermodynamical equilibrium if it cannot spontaneously undergo a finite change to another state without a finite change in the state of the environment ${ }^{6}$. A thermodynamic process involves a change in the state of the system. Classical thermodynamics models processes that occur between stable equilibrium states, and is limited to state variables that pertain to stable equilibrium only. A reversible process between two stable states takes place along a succession of quasi-stable states each of which is infinitesimally apart from the next. Hence a reversible process proceeds

\footnotetext{
${ }^{6}$ Environment is defined as the space outside the boundaries of the system.
} 
infinitely slowly. Any process which starts or takes place away from equilibrium or in a finite time is an irreversible process (Haywood, 1980). An irreversible process can take place in one direction only (thereby giving a thermodynamical definition to "time") and involves loss of energy. Practically all processes in nature occur within a finite time and exhibit varied degrees of irreversibility.

Let us consider a system $\mathcal{R}$ defined by the closed ${ }^{8}$ boundary $\partial \mathcal{R}$ (see Fig. 3.3 ). Throughout this chapter and the rest of this report, we shall assume that $\mathcal{R}$ is in contact with a heat reservoir whose (constant) absolute temperature is $\theta$. Let $W$ be the work. done on $\mathcal{R}$, and let $U$ and $K_{E}$ be its internal and kinetic energies respectively. The rate of heat flow into the system from the surrounding reservoir is $\dot{Q}$ and the increase in the entropy of $\mathcal{R}$ occurs at the rate of $\dot{S}$.

The first law of thermodynamics states that energy can neither be created nor be destroyed: The rate of increase in the kinetic and internal energies of a system equals the rate of the net inflow of heat plus the rate of net work done on the system:

$$
\dot{K_{E}}+\dot{U}=\dot{Q}+\dot{W}
$$

where the superscript dot indicates total derivative with respect to time. The second law of thermodynamics states that entropy can be created, but once created it can never be destroyed. The change in entropy of the reservoir due to the flow of heat out of it (at the constant temperature $\theta$ ) is

$$
\dot{S}_{r e s}=\frac{-\dot{Q}}{\theta}
$$

The above relation holds whether the flow of heat is reversible or not, since entropy is a state function and the reservoir is large enough to attain the same state irrespective of the nature of the process which solely involves flow of heat. The rate of increase of entropy of the universe (the system and the reservoir in this case) is then the sum of $\dot{S}_{\text {res }}$ and $\dot{S}$, which has to be non-negative by the second law of thermodynamics:

$$
\dot{S}-\frac{\dot{Q}}{\theta} \geq 0
$$

The total entropy is preserved (i.e., the change in entropy is zero) only in the case of a reversible process.

\subsubsection{The Dissipation Rate}

The rate of dissipation can be derived in terms of the Helmholtz free energy, $\Psi$, which is defined as:

$$
\Psi=U-\theta S
$$

\footnotetext{
${ }^{7}$ If an equation describing a time-dependent physical process is invariant to the algebraic sign of the variable time, it is called reversible. Otherwise it is irreversible (Prigogine, 1967).

${ }^{8} \mathrm{~A}$ closed boundary is one which allows transfer of energy but not mass.
} 
The decrease in $\Psi$, in the absence of dynamic effects $\left(\Delta K_{E}=0\right)$, gives an upper limit to the work output in a process between two equilibrium states of the system at the same temperature (Sears and Salinger, 1975):

$$
\begin{aligned}
-\Delta \Psi & =-\Delta U+\theta \Delta S \\
& \geq-\Delta U+\Delta Q \\
& \geq-\Delta W
\end{aligned}
$$

The upper limit is attained when the process is reversible and the work is maximum. Otherwise, if the process is irreversible, the work is less than maximum. Thus the decrease in $\Psi$ gives an estimate of the maximum work that can be freed from the system.

Let $\Psi$ be a function

$$
\Psi=\Psi(\theta, \mathbf{x}, \mathbf{D})
$$

where $\mathbf{D}$ is the damage tensor and $\mathbf{x}$ is the remaining set of variables required to describe the free energy. The rate of change of Helmholtz free energy can be written as

$$
\dot{\Psi}=\dot{U}-\theta \dot{S}-S \dot{\theta}
$$

which can be combined with the first law (eq 3.27) and incorporated into the second law inequality (3.29) as

$$
-\dot{K}_{E}-\dot{\Psi}-\dot{\theta} S+\dot{W} \geq 0
$$

The rate of change in $\Psi$ can be expressed in terms of $\dot{\theta}, \dot{\mathbf{x}}$ and $\dot{\mathbf{D}}$ as,

$$
\dot{\Psi}=\frac{\partial \Psi}{\partial \theta} \dot{\theta}+\frac{\partial \Psi}{\partial \mathbf{x}} \cdot \dot{\mathbf{x}}+\frac{\partial \Psi}{\partial \mathbf{D}} \cdot \dot{\mathbf{D}}
$$

with which we can rewrite the inequality $(3.34)$ as

$$
-\dot{K}_{E}-\left(\frac{\partial \Psi}{\partial \theta}+S\right) \dot{\theta}+\dot{W}-\frac{\partial \Psi}{\partial \mathbf{x}} \cdot \dot{\mathbf{x}}-\frac{\partial \Psi}{\partial \mathbf{D}} \cdot \dot{\mathbf{D}} \geq 0
$$

Noting that $\dot{\theta}=0$ this can be reduced to

$$
-\dot{K}_{E}+\dot{W}-\frac{\partial \Psi}{\partial \mathbf{x}} \cdot \dot{\mathbf{x}}-\frac{\partial \Psi}{\partial \mathbf{D}} \cdot \dot{\mathbf{D}} \geq 0
$$

The above inequality is the general statement of the second law of thermodynamics for a system that is in diathermal contact ${ }^{9}$ with a heat reservoir. We denote its left hand side as the dissipation rate, $\Gamma$ :

$$
\Gamma \equiv \dot{W}-\dot{K}_{E}-\frac{\partial \Psi}{\partial \mathbf{x}} \cdot \dot{\mathbf{x}}-\frac{\partial \Psi}{\partial \mathbf{D}} \cdot \dot{\mathbf{D}} \geq 0
$$

\footnotetext{
${ }^{9}$ Heat can flow freely through a diathermal boundary.
} 
The rate of change in kinetic energy is in fact $\mathbf{p} \cdot \dot{\mathbf{v}}$, where $\mathbf{p}$ and $\dot{\mathbf{v}}$ are the momenturn and acceleration, respectively. The rate of work, $\dot{W}$, done on the system depends on the particular system and process under consideration. It could, for example, be $F \dot{L}$ (for a wire in tension), $-P \dot{V}$ (for a gas), $E \dot{I}$ (in an electric field) or $H \dot{M}$ (in a magnetic field) or a combination thereof. The general expression for $\dot{W}$ is therefore of the form $\mathbf{Y} \cdot \dot{\mathbf{x}}$.

If the dissipation rate, $\Gamma$, is zero, the process is reversible. As a simple example, it can be verified that for a rigid body not undergoing damage accumulation (i.e., $\dot{\mathbf{x}}=\mathbf{0}, \dot{\mathbf{D}}=$ 0), a reversible process implies $\Gamma=\dot{W}-\dot{K}_{E}=0$, which says that non-frictional and non-gravitational work done on it is fully converted to kinetic energy, a fact well-known in mechanics.

\subsection{Damage Growth in Deformable Bodies}

Thus far, the above development was kept general insofar as the thermodynamic systern in consideration was in diathermal contact with a heat reservoir at constant temperature. Let us now specify the system $\mathcal{R}$ as a deformable body in contact with a heat reservoir at constant temperature. The set of variables $x$ in the free energy (eq 3.32) is then the symmetric strain tensor $\epsilon_{i j}$, defined by

$$
\epsilon_{i j}=\frac{1}{2}\left(u_{i, j}+u_{j, i}\right)
$$

where $u_{i}$ is the deformation at a point, and $u_{i, j}$ refers to its partial derivative w.r.t. the $j$ displacement. The velocity and acceleration at the point are denoted by $\dot{u}_{i}$ and $a_{i}$ respectively. The strain rate tensor is given by

$$
\dot{\epsilon}_{i j}=\frac{1}{2}\left(\dot{u}_{i, j}+\dot{u}_{j, i}\right)
$$

The stress tensor $\sigma_{i j}$ is the partial derivative of the free energy per unit volume with regard to the strain tensor (Maugin, 1992):

$$
\frac{\partial \psi}{\partial \epsilon_{i j}}=\sigma_{i j}
$$

where

$$
\psi=\frac{\partial \Psi}{\partial V}
$$

is the free energy per unit volume. The stress tensor is symmetric, i.e., $\sigma_{i j}=\sigma_{j i}$, as can be verified easily.

Classical equilibrium thermodynamics (also called thermostatics) defines state variables (e.g., $\theta, S, U$ and $\Psi$ ) and stationarity principles for systems in equilibrium or undergoing reversible processes only. However, reversibility is an idealized condition, and all processes occurring in nature are irreversible to some extent. Since the first half of 
this century, researchers have sought to extend the domain of applicability of equilibrium thermodynamics by ascribing quantities like temperature and entropy to non-equilibrium states, under various postulates and axioms (e.g., de Groot and Mazur, 1962; Prigogine, 1967; Truesdell, 1984; Sieniutycz and Salamon, 1990). There is, however, no consensus in the scientific community about the interpretation of non-equilibrium thermodynamics and the precise definition of the state variables away from equilibrium, and they continue to be areas of active research. For the present purpose of modeling accumulation of damage in deformable bodies, it is assumed that (i) the initial state of the system at time $t_{1}$ is a thermodynamic equilibrium state, and (ii) the damage-causing processes are slow processes, and though irreversible, proceed sufficiently close to equilibrium.

The work done on a system in a dissipative process at constant temperature (i.e., when the system is in contact with a single reservoir at this temperature, for example, a steady environment), minus the increase in kinetic energy, is greater than the increase in the Helmholtz free energy. Therefore,

$$
d \Psi=d^{*} W_{n k}-d^{*} W_{d} ; \quad d^{*} W_{d} \geq 0
$$

Let the dissipation in the above process be denoted by $d^{*} W_{d}$ where $d^{*} W_{n k}$ and $d^{*} W_{d}$ denote the non-kinetic work and the dissipation in the above process. The asterisks in the above differentials emphasize that they are imperfect differentials, meaning that their integrations do not necessarily vanish around a closed path. Integrating the above equation, the free energy can be expressed as:

$$
\Psi=\int d^{*} W_{n k}-\int d^{*} W_{d}
$$

A system in diathermal contact with a heat reservoir is in a state of equilibrium (not necessarily stable ${ }^{\mathbf{1 0}}$ ) if the first variation of its Helmholtz Free Energy is zero (McLellan, 1980):

$$
\delta \Psi=0
$$

The variation in the Free Energy at an arbitrary instant $t_{2}$ can be written as (cf eq $3.5)$

$$
\begin{aligned}
\delta \Psi\left(t_{2}\right) & =\delta \Psi\left(t_{1}\right)+\delta \int_{t_{1}}^{t_{2}}\left(\dot{W}-\dot{K}_{E}\right) d t-\delta \int_{t_{1}}^{t_{2}} \Gamma d t \\
& =\delta \int_{t_{1}}^{t_{2}}\left(\dot{W}-\dot{K}_{E}\right) d t-\delta \int_{t_{1}}^{t_{2}} \Gamma d t
\end{aligned}
$$

\footnotetext{
${ }^{10} \mathrm{~A}$ system is in stable thermodynamical equilibrium if it cannot spontaneously change its state to another without a finite change in its environment. In the absence of external stimuli, the above system can change its current state to another only if the latter state has a lower free energy (Callen, 1988). In other words, if the free energy has a minimum at some state, then it is a state of stable equilibrium. Mathematically, the condition of stable equilibrium is (McLellan, 1980): $\delta^{2} \Psi \geq 0$
} 
where the energy dissipation is assumed to be given by eq (3.38) and the initial state is assumed to be one of thermodynamic equilibrium. The variation $\delta \Psi\left(t_{2}\right)$ is in general a function

$$
\delta \Psi\left(t_{2}\right)=g\left(\theta, \epsilon_{i j}, \mathbf{D}, \delta \dot{\theta}, \delta \dot{\epsilon}_{i j}, \delta \dot{\mathbf{D}}, \ldots ; t\right), \quad t \in\left[t_{1}, t_{2}\right]
$$

which depends on the state of the system as well as on the choice of the variations in temperature, strain rate, damage and other terms, and is generally non-zero for an irreversible process or for a system yet to achieve equilibrium. However, slow damage growth prior to localization of defects is assumed to occur close to equilibrium and the function $g(\cdot)$ is assumed to vanish for a suitable set of variations. Under this assumption, we can write (using eq 3.38 ):

$$
\begin{aligned}
\delta \Psi\left(t_{2}\right) & =\delta \int_{t_{1}}^{t_{2}}\left(\dot{W}-\dot{K}_{E}\right) d t-\delta \int_{t_{1}}^{t_{2}} \Gamma d t \\
& =\delta \int_{t_{1}}^{t_{2}}\left(\dot{W}-\dot{K}_{E}\right) d t-\delta \int_{t_{1}}^{t_{2}}\left(\dot{W}-\dot{K}_{E}-\frac{\partial \Psi}{\partial \epsilon_{i j}} \dot{\epsilon}_{i j}-\frac{\partial \Psi}{\partial \mathbf{D}} \dot{\mathbf{D}}\right) d t \\
& =0
\end{aligned}
$$

The validity of this assumption will be tested with the accuracy of the resulting models of damage accumulation (in Chapters 4 and 5). The above equation can be rearranged as:

$$
\begin{aligned}
\delta \Psi\left(t_{2}\right) & =\delta \int_{t_{1}}^{t_{2}}\left(\dot{W}-\dot{K}_{E}+\frac{\partial \Psi}{\partial \mathbf{D}} \dot{\mathbf{D}}\right) d t-\delta \int_{t_{1}}^{t_{2}}\left(\dot{W}-\dot{K}_{E}-\frac{\partial \Psi}{\partial \epsilon_{i j}} \dot{\epsilon}_{i j}\right) d t \\
& =\delta \int_{t_{1}}^{t_{2}} I_{1}(t) d t-\delta \int_{t_{1}}^{t_{2}} I_{2}(t) d t \\
& =\int_{t_{1}}^{t_{2}} \delta I_{1}(t) d t-\int_{t_{1}}^{t_{2}} \delta I_{2}(t) d t \\
& =0
\end{aligned}
$$

where $I_{1}$ and $I_{2}$ refer to the two integrands in eq (3.48) and the commutability of integration and variation has been used.

Let us consider the second variational term first. Body forces $F_{i}$ and boundary traction $T_{i}$ perform work on the system acting through the displacement field $u_{i}$ on $\mathcal{R}$ and $\partial \mathcal{R}$, respectively. Suppose $\partial \mathcal{R}$ can be described as the union of two disjoint sets $\partial \mathcal{R}_{1}$ and $\partial \mathcal{R}_{2}$ such that displacements on only $\partial \mathcal{R}_{2}$ are specified at all times in $\left[t_{1}, t_{2}\right]$. Apply a small variation $\delta \dot{u}_{i}$ consistent with the boundary conditions

$$
\delta \dot{u}_{i}=0 \quad \text { on } \partial \mathcal{R}_{2} \quad \forall t \in\left[t_{1}, t_{2}\right]
$$


on the velocity field of the system such that it does not alter the instantaneous force, acceleration and stress distributions. The resulting instantaneous variation in $I_{2}(t)$ in eq $(3.49)$ is

$$
\begin{aligned}
\delta I_{2}(t) & =\delta\left(\dot{W}-\dot{K}_{E}-\frac{\partial \Psi}{\partial \mathbf{x}} \dot{\mathbf{x}}\right) \\
& =\int_{\mathcal{R}} F_{i} \delta \dot{u}_{i} d V+\int_{\partial \mathcal{R}_{1}} T_{i} \delta \dot{u}_{i} d \eta-\int_{\mathcal{R}} \rho a_{i} \delta \dot{u}_{i} d V-\int_{\mathcal{R}} \sigma_{i j} \delta \dot{\epsilon}_{i j} d V \\
& =\int_{\mathcal{R}} F_{i} \delta \dot{u}_{i} d V+\int_{\partial \mathcal{R}} T_{i} \delta \dot{u}_{i} d \eta-\int_{\mathcal{R}} \rho a_{i} \delta \dot{u}_{i} d V-\int_{\mathcal{R}} \sigma_{i j} \delta \dot{u}_{i, j} d V
\end{aligned}
$$

where use has been made of the symmetry of the stress tensor. Upon integration by parts,

$$
\begin{aligned}
\delta I_{2}(t) & =\int_{\mathcal{R}} F_{i} \delta \dot{u}_{i} d V+\int_{\partial \mathcal{R}} T_{i} \delta \dot{u}_{i} d \eta-\int_{\mathcal{R}} \rho a_{i} \delta \dot{u}_{i} d V-\int_{\mathcal{R}}\left(\sigma_{i j} \delta \dot{u}_{i}\right)_{, j} d V+\int_{\mathcal{R}} \sigma_{i j, j} \delta \dot{u}_{i} d V \\
& =\int_{\mathcal{R}}\left(F_{i}+\sigma_{i j, j}-\rho a_{i}\right) \delta \dot{u}_{i} d V+\int_{\partial \mathcal{R}} T_{i} \delta \dot{u}_{i} d \eta-\int_{\mathcal{R}}\left(\sigma_{i j} \delta \dot{u}_{i}\right)_{, j} d V
\end{aligned}
$$

Applying Green's theorem to the last integral,

$$
\begin{aligned}
\delta I_{2}(t) & =\int_{\mathcal{R}}\left(F_{i}+\sigma_{i j, j}-\rho a_{i}\right) \delta \dot{u}_{i} d V+\int_{\partial \mathcal{R}} T_{i} \delta \dot{u}_{i} d \eta-\int_{\partial \mathcal{R}} \sigma_{i j} n_{j} \delta \dot{u}_{i} d \eta \\
& =\int_{\mathcal{R}}\left(F_{i}+\sigma_{i j, j}-\rho a_{i}\right) \delta \dot{u}_{i} d V+\int_{\partial \mathcal{R}}\left(T_{i}-\sigma_{i j} n_{j}\right) \delta \dot{u}_{i} d \eta
\end{aligned}
$$

Since $\delta \dot{u}_{i}$ is identically zero on $\partial \mathcal{R}_{2}$, the above expression is equal to

$$
\delta I_{2}(t)=\int_{\mathcal{R}}\left(F_{i}(t)+\sigma_{i j, j}(t)-\rho a_{i}(t)\right) \delta \dot{u}_{i}(t) d V+\int_{\partial \mathcal{R}_{1}}\left(T_{i}(t)-\sigma_{i j}(t) n_{j}\right) \delta \dot{u}_{i}(t) d \eta
$$

The expressions in parentheses in the above integrals are each zero as they constitute the equilibrium equations of the damaged body on $\mathcal{R}$ and $\partial \mathcal{R}_{1}$ respectively (Krajcinovic and Sumarac, 1987). Hence the variation of the first integral in eq (3.49),

$$
\delta \int_{t_{1}}^{t_{2}} I_{1}(t) d t=\delta\left[\int_{t_{1}}^{t_{2}}\left(\dot{W}-\dot{K}_{E}+\frac{\partial \Psi}{\partial \mathbf{D}} \dot{\mathbf{D}}\right) d t\right]=0
$$

It should be recalled that the above condition (3.54) arises from the assumption that the damage causing processes are slow and proceed sufficiently close to equilibrium in the pre-localization state. The extent of validity of this assumption will be reflected on the accuracy of the damage models developed in Chapters 4 and 5 . It should also be noted that this relation is restricted to materials and processes for which the Helmholtz free energy (3.32) can be suitably defined. 
Under the above assumptions and restrictions, it is now possible to apply appropriate sets of variations on the deformable body in equilibrium (consistent with the boundary conditions used to derive eq 3.53) and to use eq (3.54) to derive partial differential equations of damage growth. Constitutive equations relevant to a given damage-causing mechanism can then be incorporated into these differential equations, and corresponding damage growth laws may be obtained. This scheme will be implemented in chapter 4 , under the assumption of isotropic damage, where damage growth laws for ductile deformation, high-temperature creep and fatigue will be derived. 


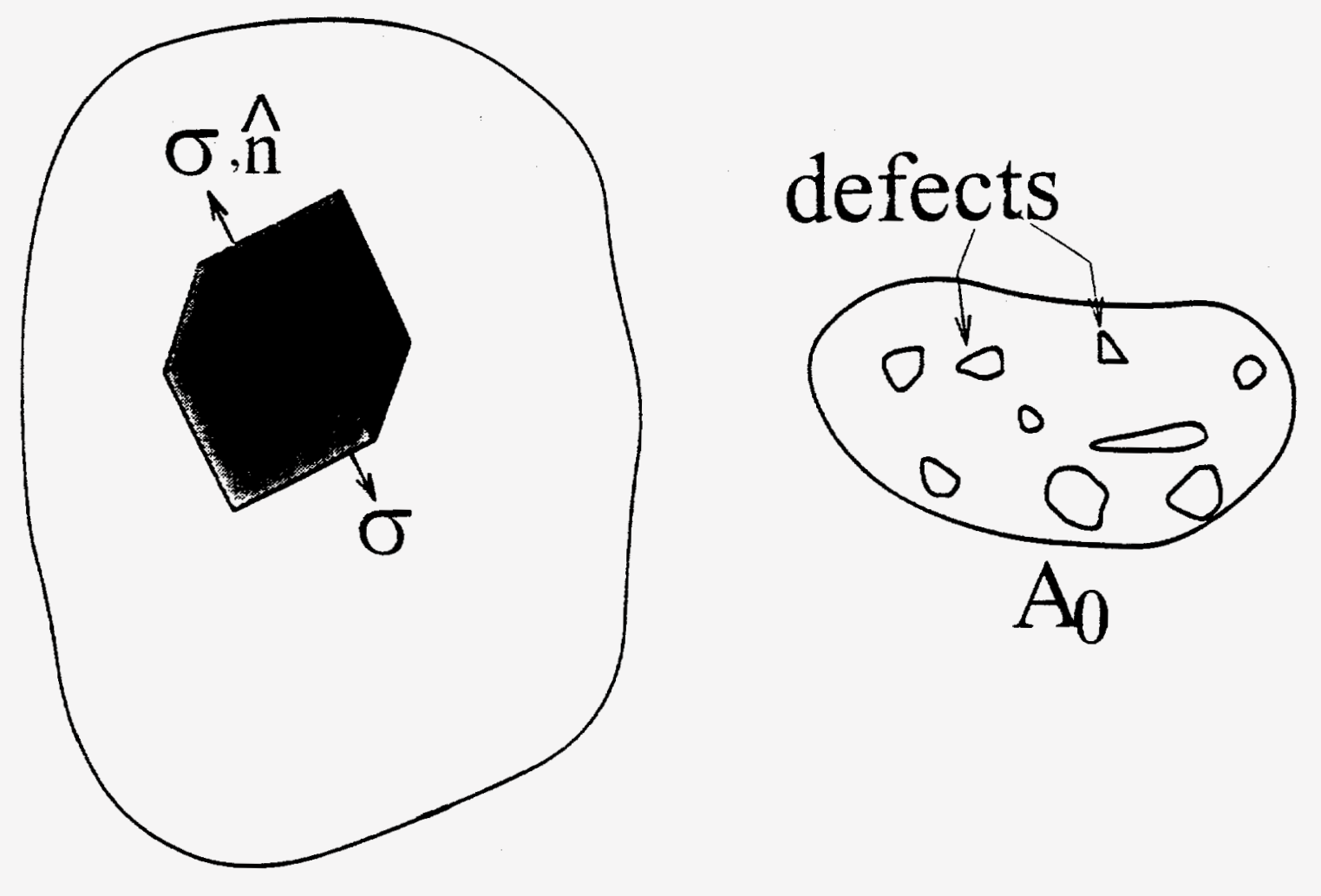

Figure 3.1: The damage variable 

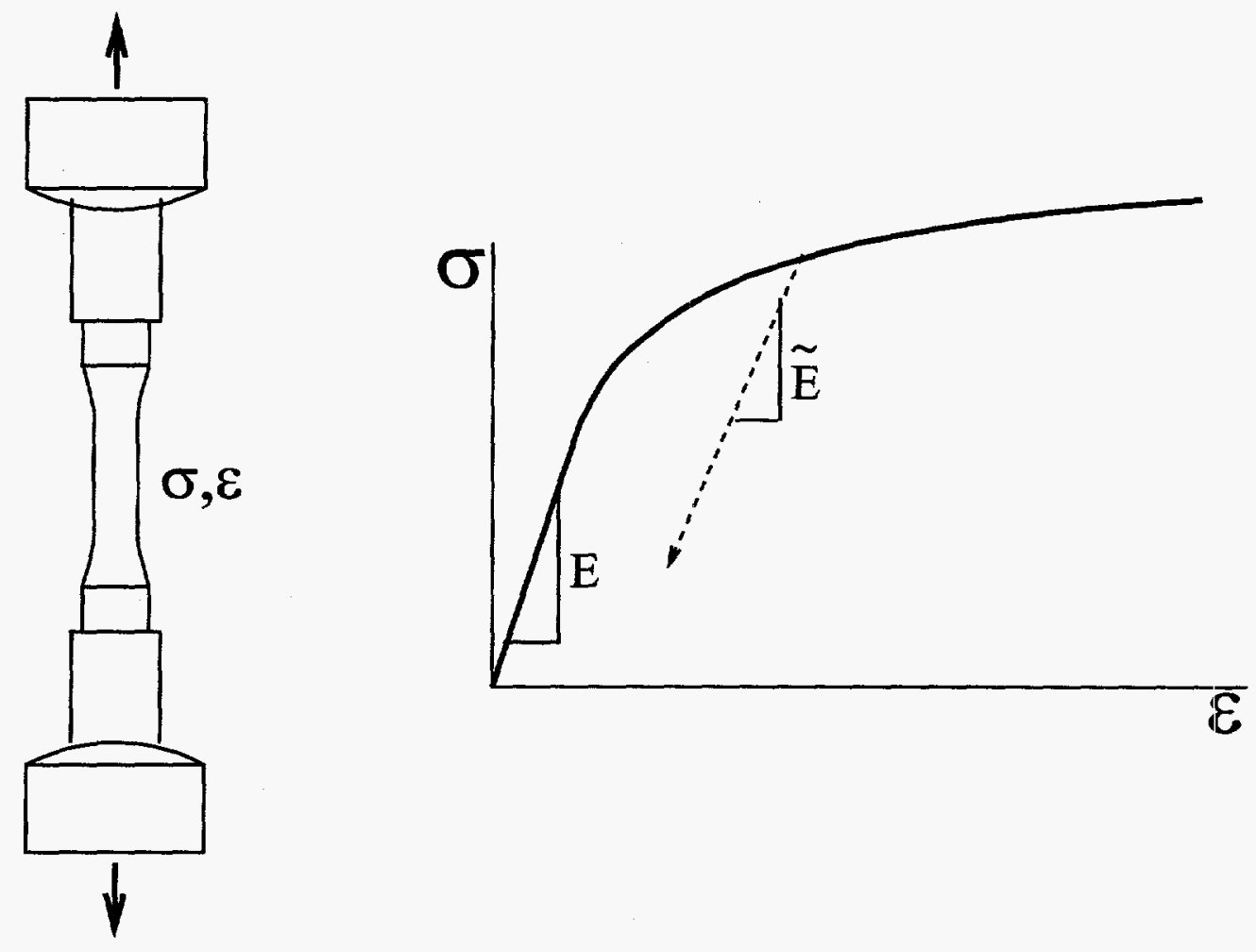

Figure 3.2: Experimental determination of damage 


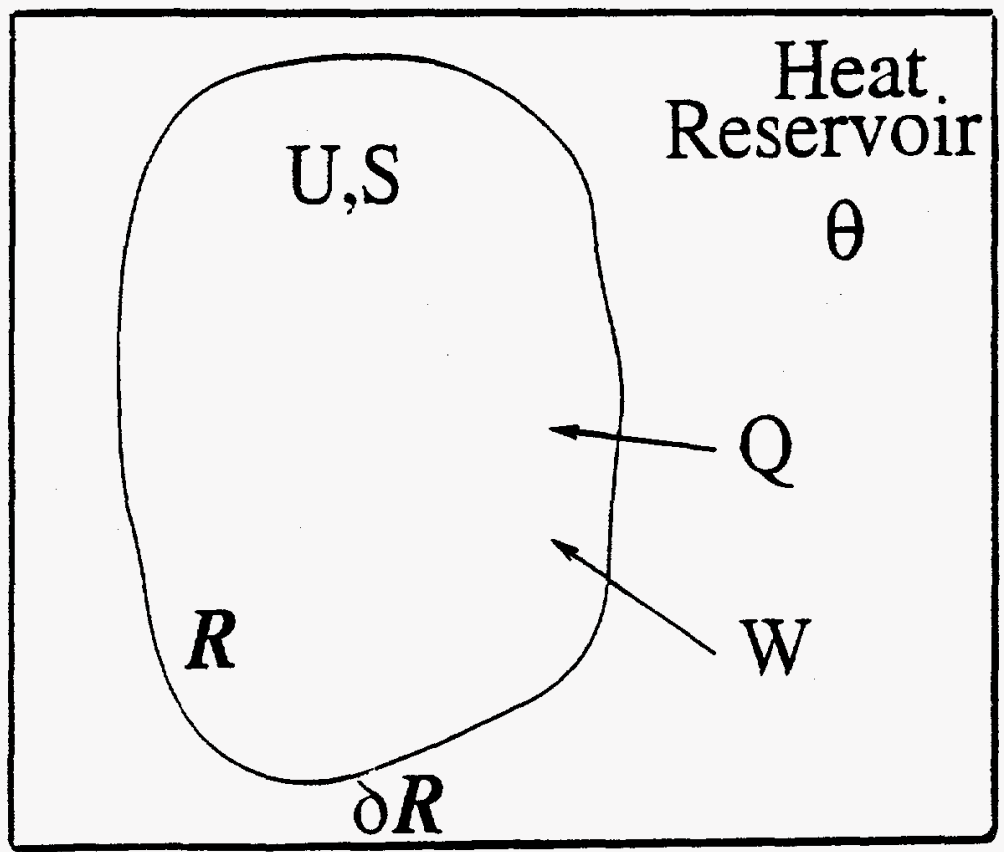

Figure 3.3: State variables and energy exchange for a closed system 



\section{Chapter 4}

\section{A Unified Model for Isotropic Damage Growth}

In the previous chapter, the fundamental principles of continuum damage mechanics were introduced. The thermodynamics of damage accumulation was described and a stationarity condition was developed under a set of stated assumptions. In the present chapter, this stationarity condition will be shown to lead to a set of partial differential equations of isotropic damage growth which, with the help of relevant constitutive equations, can be applied to any damage causing mechanism so long as it remains consistent with the notions of continuum damage mechanics and allows a suitable expression for the Helmholtz free energy. This unified approach will be applied to three damage growth processes, namely ductile deformation, creep and fatigue, and the models will be verified with published experimental results.

The behavior of a metal under uniaxial loading (as opposed to multiaxial loading) is the most common way to characterize its mechanical behavior, and material properties and experimental data are also most commonly available in terms of uniaxial deformation. The focus in this report is therefore on material response under uniaxial loading.

\subsection{The Isotropic Damage Growth Model}

Damage is considered isotropic in the following, as this will simplify the notations without loss of generality. It will be consistent with available experimental data, most of which are on the scalar damage variable. Since damage is observed to accumulate in the presence of straining, it is reasonable to suppose that damage can be expressed as function of the strain tensor. Let us assume that the variation of the integrand in eq (3.54) vanishes at every instant,

$$
\delta I_{1}(t)=\delta\left(\dot{W}(t)-\dot{K}_{E}(t)+\frac{\partial \Psi}{\partial D} \dot{D}\right)=0
$$


and apply a set of variations similar to that applied to eq (3.53) and obeying the same boundary conditions. In particular, a small variation $\delta \dot{u}_{i}(t)$ subject to boundary condition (3.50) is applied on the velocity field of the system $\mathcal{R}$ such that it does not alter the instantaneous force, acceleration and strain distributions; nor does it affect the rate of: change in the free energy, $\partial \psi / \partial D$, at that instant. The resulting variation in eq (4.1) is

$$
\int_{\mathcal{R}} F_{i} \delta \dot{u}_{i} d V+\int_{\partial \mathcal{R}_{1}} T_{i} \delta \dot{u}_{i} d \eta-\int_{\mathcal{R}} \rho a_{i} \delta \dot{u}_{i} d V+\int_{\mathcal{R}} \frac{\partial \psi}{\partial D} \delta \dot{D} d V=0
$$

The commutability of variation and differentiation,

$$
\delta \dot{D}=\frac{d}{d t} \delta D
$$

may be used in eq (4.2) to obtain

$$
\int_{\mathcal{R}} F_{i} \delta \dot{u}_{i} d V+\int_{\partial \mathcal{R}_{1}} T_{i} \delta \dot{u}_{i} d \eta-\int_{\mathcal{R}} \rho a_{i} \delta \dot{u}_{i} d V+\int_{\mathcal{R}} \frac{\partial \psi}{\partial D} \frac{d}{d t}\left(\frac{\partial D}{\partial \epsilon_{i j}} \delta \epsilon_{i j}\right) d V=0
$$

Noting that the applied variation is such that $\delta \epsilon_{i j}=0$, we have, by the chain rule of differentiation,

$$
\int_{\mathcal{R}} F_{i} \delta \dot{u}_{i} d V+\int_{\partial \mathcal{R}_{1}} T_{i} \delta \dot{u}_{i} d \eta-\int_{\mathcal{R}} \rho a_{i} \delta \dot{u}_{i} d V+\int_{\mathcal{R}} \frac{\partial \psi}{\partial D} \frac{\partial D}{\partial \epsilon_{i j}} \delta \dot{\epsilon}_{i j} d V=0
$$

As the strain tensor is symmetric, $\partial D / \partial \epsilon_{i j}$ is symmetric with respect to $i, j$. Integrating by parts, applying Green's theorem as before, and noting the boundary condition (3.50), equation (4.5) can be written as:

$$
\delta I_{1}(t)=\int_{\mathcal{R}}\left(F_{i}-\left(\psi_{D} D_{i j}^{\prime}\right)_{, j}-\rho a_{i}\right) \delta \dot{u}_{i} d V+\int_{\partial \mathcal{R}_{1}}\left(T_{i}+\psi_{D} D_{i j}^{\prime} n_{j}\right) \delta \dot{u}_{i} d \eta=0
$$

where $D_{i j}^{\prime}=\partial D / \partial \epsilon_{i j}$. We may now choose the variation in the velocity field which satisfy $\delta \dot{u}_{i}(t)=0$ on $\partial \mathcal{R}_{1}$, which implies

$$
F_{i}-\rho a_{i}-\left(\psi_{D} D_{i j}^{\prime}\right)_{, j}=0 \text { on } \mathcal{R}
$$

We then choose non-zero variations on the free surface $\delta \dot{u}_{i}(t) \neq 0$ on $\partial \mathcal{R}_{1}$, which implies,

$$
T_{i}+\psi_{D} D_{i j}^{\prime} n_{j}=0 \text { on } \partial \mathcal{R}_{1}
$$

Eqs (4.7) and (4.8) are the equations of damage growth in a deformable body and are applicable to any mechanism of damage accumulation that is consistent with the underlying notions of CDM and has an appropriate expression of the Helmholtz free energy. The specific damage growth laws arising out of these equations will depend on the functional form of $\psi$ which, in turn, depends on the corresponding physical condition governing the stress-strain relation, viscous effects, micro-void growth and so on. This highlights the powerful and general nature of the proposed approach and suggests the possibility of expressing damage caused by different processes acting simultaneously using one unified 
damage variable. In the case of multiaxial straining, the present approach would involve the solution of a set of coupled partial differential equations (PDEs) which may become computationally prohibitive. However, the majority of the existing material characterizations and test data, as mentioned at the beginning of this chapter, are available in terms of uniaxial deformation; accordingly, and the rest of this report will focus on uniaxial loading which will not require the solution of coupled PDE's.

For damage growth under uniaxial loading due to a far-field stress, $\sigma_{\infty}$, acting normal to the surface, eq (4.8) simplifies to:

$$
\sigma_{\infty}+\psi_{D} \frac{d D}{d \epsilon}=0
$$

or,

$$
\frac{d D}{d \epsilon}=-\frac{\sigma_{\infty}}{\psi_{D}}
$$

If the strain rate is known, damage growth can be expressed as a function of time:

$$
\frac{d D}{d t}=-\frac{\sigma_{\infty}}{\psi_{D}} \dot{\epsilon}(D, t)
$$

the strain rate, $\dot{\epsilon}$, being generally a function of time as well as damage. However, in some situations the strain rate may remain constant, as in a displacement-controlled tension test.

We will subsequently investigate different modes of damage growth in deformable bodies, namely ductile deformation, high temperature creep and fatigue under cyclic loading, and derive equations for damage growth in each of these cases. The basic equation for damage growth (eq 4.9 or 4.11 ) remains the same but the free energy per unit volume, $\psi$, and its partial derivative with respect to $D$ of course assume different forms for different damage mechanisms. The general form of $\psi$ applicable to ductile deformation, constant-stress creep and fatigue cycling is

$$
\psi=\int \sigma_{i j} d \epsilon_{i j}-\gamma(D)
$$

where $\gamma$ is the surface energy of voids and discontinuities that arise due to damage growth per unit volume. Note that eq (3.41) is satisfied. Under uniaxial straining, eq (4.12) simplifies to

$$
\psi=\int \sigma d \epsilon-\gamma(D)
$$

In the general case, the increment in strain $d \epsilon$ has three additive constituents: the elastic, plastic and creep strains.

Equations (4.10 or 4.11 ) and (4.13) will be the starting point for developing isotropic damage growth equations under uniaxial loading in the remaining sections in this chapter, and will also be used in stochastic damage growth in the next chapter. The implementation of these two equations requires knowledge of the constitutive model (for a given 
loading mode) and the surface energy, $\gamma$. The constitutive models will be described individually in the following subsections for ductile deformation, creep and fatigue. The surface energy, which is common to all three, can be obtained under the following set of idealizations consistent with the notion of isotropic damage.

The defects within the material are assumed to be spherical voids (of different sizes) which are distributed uniformly in space within the material. Consider a representative volume element, $V_{0}=(4 / 3) \pi u^{3}$, in the shape of a sphere of radius $u$. Suppose there are $n$ voids present in this volume and $a_{i}$ is the radius of the $i^{\text {th }}$ void $(i \in[1, n])$. If $D$ is the state of damage on some cross-section of area $S_{0}$ within $V_{0}$ and if $n$ is large, then, ignoring stress-amplification, $D S_{0}$ is the net surface area of defects on that cross-section. Hence, $\left(D S_{0}\right) d y$ is the volume of defects in a wafer whose thickness is $d y$. Integrating over the entire volume of the sphere of radius $u$, the volume of defects present in that sphere is found to be $\frac{4}{3} \pi u^{3} D$. This is also equal to the sum of the individual void volumes:

$$
\frac{4}{3} \pi u^{3} D=\sum_{i=1}^{n} \frac{4}{3} \pi a_{i}^{3}
$$

which gives

$$
D=\frac{1}{u^{3}} \sum_{i=1}^{n} a_{i}^{3}
$$

Assuming a linear force-displacement relation at the microscale, and that a void is formed when the stress on its impending boundary equals the local failure stress, $\sigma_{c}$, the energy required to form the $i^{\text {th }}$ void is

$$
\Pi_{i}=\int_{\eta} \frac{1}{2} \sigma_{c} v d \eta
$$

where $d \eta$ is an elemental void boundary and $v$ is the extension normal to the initial circular plane which finally expands to the spherical void.

For a sphere of radius $a_{i}$ the above potential is equal to

$$
\begin{aligned}
\Pi_{i} & =2 \sigma_{c} \int_{0}^{a_{i}} \frac{1}{2} \sqrt{a_{i}^{2}-x^{2}} 2 \pi x d x \\
& =\frac{2}{3} \sigma_{c} \pi a_{i}^{3}
\end{aligned}
$$

The surface energy required per unit growth of void surface area (assuming that the void grows when the stress on its boundary equals the local failure stress $\sigma_{c}$ ) is:

$$
\frac{\partial \Pi}{\partial \eta}=\frac{\partial \Pi}{\partial a_{i}} \frac{\partial a_{i}}{\partial \eta}=\frac{1}{4} \sigma_{c} a_{i}
$$

Summing over all the $n$ voids, the total surface energy is

$$
\sum_{i=1}^{n}\left(\frac{\sigma_{c} a_{i}}{4}\right) 4 \pi a_{i}^{2}
$$


Hence the surface energy per unit volume of the material is,

$$
\gamma=\frac{\sigma_{c} \sum_{i=1}^{n} \pi a_{i}^{3}}{V_{0}}=\frac{\sigma_{c} \sum_{i=1}^{n} \pi a_{i}^{3}}{\frac{4}{3} \pi u^{3}}
$$

With the help of eq (4.15) this becomes

$$
\gamma=\frac{3}{4} \sigma_{c} D
$$

The local failure stress $\sigma_{c}$ may be relatively difficult to obtain experimentally, but its value is assumed to be close to the true fracture strength, $\sigma_{f}$, of the material, which is an easily available material property. In the sequel, $\gamma$ is evaluated as

$$
\gamma=\frac{3}{4} \sigma_{f} D
$$

The validity of this assumption will be tested in sec 4.2 .2 .

It should be noted that the above approach represents a naive way of estimating the surface energy of formation of voids. Nevertheless, it provides the estimate (eq 4.21) in terms of easily known quantities. A more comprehensive approach would probably introduce a greater level of complexity, and while being more realistic and accurate, would possibly contain variables like the number, shapes and sizes of the voids as a function of time (or strain) whose numerical estimates would not always be readily available.

Finally, the equations of damage growth under ductile deformation, creep and fatigue and a knowledge of the critical damage parameter may be used to determine the remaining service life and residual strength of a structure. The following postulate defines the critical damage (eg, Chow and Wei, 1991):

The (temperature-dependent) critical damage parameter, $D_{c}$, is a material property that is independent of the loading history. For any combination of loads occurring in any sequence, a structure fails when the accumulated damage $D$ equals the critical value $D_{c}$.

The above postulate gives us a tool to predict failure in a complex loading situation where the critical damage for that particular loading condition is not known a priori. The same value of $D_{c}$ for a given material at a particular temperature ascertained from a simple test (eg, a static tension test), can be used in other situations (eg, variable amplitude loading) to predict the time to failure (or equivalently, plastic strain to failure, or number of cycles to failure) when the accumulated damage equals the critical value $D_{c}$.

The remaining three subsections of this chapter develop isotropic damage growth models for ductile deformation, high-temperature creep and fatigue, starting from the general equations developed thus far. The models are verified in each case with published experimental results and sensitivity studies are also presented. It may be noted that, unlike the existing CDM-based methods of damage growth, only commonly available material properties enter the proposed equations of damage growth. 


\subsection{Ductile Deformation Damage}

\subsubsection{Damage Growth Models}

Consider a component subjected to a uniaxial, monotonically increasing load. The first term in (eq 4.12) then takes the form

$$
\int_{0}^{\epsilon} \sigma d \epsilon^{\prime}=\int_{0}^{\epsilon_{e}} \sigma d \epsilon_{\mathrm{e}}^{\prime}+\int_{0}^{\epsilon_{p}} \sigma d \epsilon_{p}^{\prime}
$$

where total strain has been decomposed into its plastic and elastic components, $\epsilon_{p}$ and $\epsilon_{e}$ respectively. To evaluate eq (4.22) we apply the Ramberg-Osgood law for monotoric loading to the effective stress-actual strain relationship:

$$
\epsilon=\frac{\tilde{\sigma}}{E}+\left(\frac{\tilde{\sigma}}{K}\right)^{M}
$$

where $\tilde{\sigma}$ is the effective stress, $E$ is the undamaged elastic modulus, $K$ is the undamaged strain hardening modulus and $M$ is the hardening exponent. Typical values of these constants for different metals are summarized in Table 4.1. The Ramberg-Osgood law separates total strain into elastic and plastic components which are related according to

$$
\epsilon_{e}=\frac{K}{E} \epsilon_{p}^{\frac{1}{M}}
$$

Adopting the principle of strain equivalence and applying it to the elastic and plastic strains with the assumption that the exponent $M$, which determines the shape of the stress-strain curve, is not affected by damage, we obtain

$$
\epsilon_{e}= \begin{cases}\frac{\tilde{\sigma}}{E} ; & \text { under effective stress } \\ \frac{\sigma}{\tilde{E}} ; & \text { under nominal stress }\end{cases}
$$

and

$$
\left(\epsilon_{p}\right)^{\frac{1}{M}}= \begin{cases}\frac{\tilde{\sigma}}{K} ; & \text { under effective stress } \\ \frac{\sigma}{\tilde{K}} ; & \text { under nominal stress }\end{cases}
$$

in which $\tilde{E}$ and $\tilde{K}$ are, respectively, the moduli of elasticity and hardening of a damaged volume of material. It has been reported from experimental observations that damage initiates only after the accumulation of a threshold plastic strain, $\epsilon_{0}$. Therefore, the values of the stiffness moduli remain unchanged until the strain reaches the threshold value. Since $\tilde{\sigma}=\sigma /(1-D)$, the damaged moduli are linear functions of isotropic damage:

$$
\left.\begin{array}{l}
\tilde{E}=E(1-D) \\
\tilde{K}=K(1-D)
\end{array}\right\} \text { for } \epsilon_{p} \leq \epsilon_{0}, \epsilon_{e} \leq \epsilon_{e 0}
$$


where $\epsilon_{e 0}$ is an infinitesimal elastic strain corresponding to $\epsilon_{0}$ (eq 4.24), arising out of the fact that the Ramberg-Osgood model does not admit a purely elastic stress-strain relation. It is of interest to note that eq (4.27), which arises out of the principle of strain equivalence, allows the Ramberg-Osgood equation to also be expressed in terms of the damaged moduli:

$$
\epsilon=\frac{\sigma}{\tilde{E}}+\left(\frac{\sigma}{\tilde{K}}\right)^{M}
$$

Thus, this widely adopted stress-strain law is valid for both undamaged and damaged materials. It can be easily verified from eq (4.28) that the relation between the elastic and plastic strains (eq 4.24) is preserved.

The integral in the free energy (eq 4.22) can now be written as:

$$
\int_{0}^{\epsilon} \sigma d \epsilon^{\prime}=\int_{0}^{\epsilon_{e 0}} E \epsilon_{e}^{\prime} d \epsilon_{e}^{\prime}+\int_{\epsilon_{e 0}}^{\epsilon_{e}} E(1-D) \epsilon_{e}^{\prime} d \epsilon_{e}^{\prime}+\int_{0}^{\epsilon_{0}} K \epsilon_{p}^{\prime \frac{1}{M}} d \epsilon_{p}^{\prime}+\int_{\epsilon_{0}}^{\epsilon_{p}} K(1-D) \epsilon_{p}^{\frac{1}{M}} d \epsilon_{p}^{\prime}
$$

Subtracting eq (4.21) from eq (4.29) we obtain the free energy per unit volume of a damaged body subjected to uniaxial monotonic loading. Finally, the partial derivative $\psi_{D}$ can be written as:

$$
\psi_{D}=-\frac{K^{2}}{2 E}\left[\epsilon_{p}^{\frac{2}{M}}-\epsilon_{0}^{\frac{2}{M}}\right]-\frac{K}{1+\frac{1}{M}}\left[\epsilon_{p}^{1+\frac{1}{M}}-\epsilon_{0}^{1+\frac{1}{M}}\right]-\frac{3}{4} \sigma_{f}
$$

The damage growth equation can be written as:

$$
\begin{aligned}
\frac{d D}{d \epsilon} & =-\frac{\sigma_{\infty}}{\psi_{D}} \\
& =\frac{\sigma_{\infty}}{\frac{K^{2}}{2 E}\left[\epsilon_{p}^{\frac{2}{M}}-\epsilon_{0}^{\frac{2}{M}}\right]-\frac{K}{1+\frac{1}{M}}\left[\epsilon_{p}^{1+\frac{1}{M}}-\epsilon_{0}^{1+\frac{1}{M}}\right]+\frac{3}{4} \sigma_{f}}
\end{aligned}
$$

which, with the help of the principle of strain equivalence, becomes

$$
\begin{aligned}
\frac{d D}{d \epsilon} & =\frac{K(1-D) \epsilon_{p}^{\frac{1}{M}}}{\frac{K^{2}}{2 E}\left[\epsilon_{p}^{\frac{2}{M}}-\epsilon_{0}^{\frac{2}{M}}\right]+\frac{K}{1+\frac{1}{M}}\left[\epsilon_{p}^{1+\frac{1}{M}}-\epsilon_{0}^{1+\frac{1}{M}}\right]+\frac{3}{4} \sigma_{f}} \\
& =\frac{(1-D) \epsilon_{p}^{\frac{1}{M}}}{\frac{K}{2 E}\left[\epsilon_{p}^{\frac{2}{M}}-\epsilon_{0}^{\frac{2}{M}}\right]+\frac{1}{1+\frac{1}{M}}\left[\epsilon_{p}^{1+\frac{1}{M}}-\epsilon_{0}^{1+\frac{1}{M}}\right]+\frac{3}{4} \frac{\sigma_{f}}{K}}
\end{aligned}
$$

Since the left-hand side of eq (4.32) is in terms of the total strain and $\epsilon \neq \epsilon_{p}$ in general, the rate of damage growth with respect to the plastic strain may be obtained (using 
eq 4.24) as:

$$
\begin{aligned}
\frac{d D}{d \epsilon_{p}} & =\frac{d D}{d \epsilon} \frac{d \epsilon}{d \epsilon_{p}} \\
& =\frac{d D}{d \epsilon}\left(1+\frac{K}{E} \frac{1}{M} \epsilon_{p}^{1 / M-1}\right) \\
& =\frac{(1-D) \epsilon_{p}^{\frac{1}{M}}}{\frac{K}{2 E}\left[\epsilon_{p}^{\frac{2}{M}}-\epsilon_{0}^{\frac{2}{M}}\right]+\frac{1}{1+\frac{1}{M}}\left[\epsilon_{p}^{1+\frac{1}{M}}-\epsilon_{0}^{1+\frac{1}{M}}\right]+\frac{3}{4} \frac{\sigma_{f}}{K}}\left(1+\frac{K}{E} \frac{1}{M} \epsilon_{p}^{1 / M-1}\right)
\end{aligned}
$$

This ordinary differential equation describes evolution of damage as a function of plastic strain with the initial condition $D=0$ at $\epsilon_{p}=\epsilon_{0}$. In monotonic loading of ductile materials, however, the elastic strain is negligible compared to the plastic strain (particularly at strain levels where damage growth is perceptible), and consequently, the correction factor $d \epsilon / d \epsilon_{p}=1$ in eq(4.33) at all $\epsilon$ of practical interest ${ }^{1}$, and therefore $d D / d \epsilon \simeq d D / d \epsilon_{p}$ :

$$
\frac{d D}{d \epsilon_{p}}=\frac{(1-D) \epsilon_{p}^{\frac{1}{M}}}{\frac{K}{2 E}\left[\epsilon_{p}^{\frac{2}{M}}-\epsilon_{0}^{\frac{2}{M}}\right]+\frac{1}{1+\frac{1}{M}}\left[\epsilon_{p}^{1+\frac{1}{M}}-\epsilon_{0}^{1+\frac{1}{M}}\right]+\frac{3}{4} \frac{\sigma_{f}}{K}}
$$

$\mathrm{Eq}(4.34)$ is non-linear and a closed-form solution apparently does not exist. However, for metals and alloys, the elastic moduli are in general much larger than the corresponding; hardening moduli, often by two orders of magnitude. Hence for simplicity, the quantity $K /(2 E)$ may be neglected in the above differential equation:

$$
\frac{d D}{1-D}=\frac{\epsilon_{p}^{\frac{1}{M}} d \epsilon_{p}}{\frac{1}{1+\frac{1}{M}}\left[\epsilon_{p}^{1+\frac{1}{M}}-\epsilon_{0}^{1+\frac{1}{M}}\right]+\frac{3}{4} \frac{\sigma_{f}}{K}}
$$

and under this condition, the closed-form solution is:

$$
D=1-\frac{C_{2}}{\epsilon_{p}^{1+\frac{1}{M}}+C_{1}}
$$

where $C_{1}$ and $C_{2}$ are constants

$$
\begin{aligned}
& C_{1}=\frac{3}{4}\left(1+\frac{1}{M}\right) \frac{\sigma_{f}}{K}-\epsilon_{0}^{1+\frac{1}{M}} \\
& C_{2}=C_{1}+\epsilon_{0}^{1+\frac{1}{M}}
\end{aligned}
$$

\footnotetext{
${ }^{1}$ For ductile materials, the plastic strain dominates the elastic strain practically from $\epsilon=0$ all the way up to the fracture ductility, $\epsilon_{f},\left(\epsilon_{f}\right.$ is typically between 0.2 to 1.0 for engineering metals) except in the very initial stage of loading ( $\epsilon$ is typically less than 0.001 in this range) which, however, is too small to be important in ductile damage growth. Numerical evidence of this assertion is presented in the next subsection.
} 
Two further simplifications of the proposed ductile damage growth model can be obtained. The non-dimensional material constant, $C_{1}$, is greater than the plastic strain range of interest for most metals and alloys (up to the fracture ductility, $\epsilon_{f}$ ) and for such situations, eq (4.36) may be written as,

$$
D=1-\frac{C_{2}}{C_{1}}+\frac{C_{2}}{C_{1}^{2}} \epsilon_{p}^{1+\frac{1}{M}} ; \quad C_{1}>\epsilon_{p}^{1+\frac{1}{M}}
$$

For those materials that possess a marked ductility in the post-yielding zone (i.e., for those with $M \rightarrow \infty$ ), the above equation may be further simplified into a linear relation between damage and plastic strain:

$$
D=1-\frac{C_{2}}{C_{1}}+\frac{C_{2}}{C_{1}^{2}} \epsilon_{p} ; \quad C_{1}>\epsilon_{p}, \quad M \rightarrow \infty
$$

The error associated with using the above closed-form solutions instead of numerically solving eq (4.34) will be estimated subsequently.

An analytical comparison of the proposed equations with some of those already available in the literature may be insightful. Eq (4.39) is of a similar algebraic form as Lemaitre's (1985) solution for uniaxial ductile damage:

$$
\text { Lemaitre }: \quad D=D_{c}\left(\frac{\epsilon_{p}^{(2 a+M) / M}-\epsilon_{0}^{(2 a+M) / M}}{\epsilon_{f}^{(2 a+M) / M}-\epsilon_{0}^{(2 a+M) / M}}\right) ; \quad \epsilon_{p} \geq \epsilon_{0}
$$

which contains an unknown material parameter $a$, and requires a prior estimate of the failure condition $\left(\epsilon_{f}, D_{c}\right)$ to be useful. The simplification of the proposed method for highly ductile materials (eq 4.40) is comparable to Lemaitre's (1985) linear approximation:

$$
\text { Lemaitre : } \quad D=D_{c}\left(\frac{\epsilon_{p}-\epsilon_{0}}{\epsilon_{f}-\epsilon_{0}}\right) ; \quad \epsilon_{p} \geq \epsilon_{0}, M \rightarrow \infty
$$

Unlike the proposed model, Lemaitre's equation cannot be used to predict the failure condition.

The proposed differential equation of damage growth under monotonic loading (eq 4.33), or its simplified solutions, can predict damage as a function of strain and is free of any unknown material-dependent constants. The present approach requires only easily defined and available mechanical properties. It can therefore predict the critical damage, $D_{c}$, given the true failure strain, $\epsilon_{f}$. This value of $D_{c}$ may then be used to predict the time to failure in other damage mechanisms (e.g., fatigue) for the same material and temperature.

The validation of the proposed model and an investigation of its sensitivity to the material parameters are presented next. 


\subsubsection{Model Validation and Sensitivity Study}

It was noted in sec 3.2 that the reduced modulus of elasticity of a structure is a linear function of its damage state. Eq (3.9) can be inverted to estimate the isotropic damage variable by measuring the fractional change in the elastic modulus of a structure:

$$
D=1-\frac{\tilde{E}}{E}
$$

A schematic implementation of eq (4.43) has already been described in Fig 3.2. The specimen is unloaded periodically and the slope of the unloading part of the stress-strain curve gives the damaged elastic modulus. The threshold strain of damage, $\epsilon_{0}$ (if any), can also be found this way. Damage can then be plotted as a function of plastic strain.

Table 4.1 lists the monotonic material properties used for ductile damage growth in this section. It is recognized that material properties may significantly differ for the same nominal grade depending on temper and conditioning. Table 4.1 therefore lists properties from more than one source, which underscores the handicap faced in validating the method. The present model of ductile damage growth is compared with experimental data on four materials. Table 4.2 lists the predicted $D_{c}$ (solution of eq 4.34 at the given failure strain, $\epsilon_{f}$ ), and the experimental $\epsilon_{0}$ and $D_{c}$ for each of these materials. Unfortunately, the published sources (Table 4.1) of these experimental results do not list the mechanical properties of the particular specimens used in the experiments. In the absence of that information, standard properties from other published sources for the same materials (or equivalent materials for those alloys with French designations) are used.

The relative magnitude of the elastic and plastic components of total strain (obtained by solving eqs 2.2 numerically) under monotonic loading of an engineering metal is shown in Fig 4.1. The material selected for this purpose is SAE 1035 steel. The plastic strain is negligible in the initial stage (for $\epsilon<0.0002$ ), but its share of the total strain increases with increasing $\epsilon$, and the two become practically indistinguishable above $\epsilon=0.02$.

Figs. 4.2(a) and (b) show the predicted growth of ductile damage in SAE 1035 steel and compare the predictions with test results (Lemaitre, 1985) on French steel XC 38 which is equivalent ${ }^{2}$ to SAE 1035 . The difference between eqs (4.33) and $(4.34)^{3}$ is shown in Fig 4.2(a) which uses the $\log$-log scale to facilitate the comparison for small values of plastic strain. The effect of the correction factor $\left(1+d \epsilon / d \epsilon_{p}\right)$ is seen to be negligible for $\epsilon_{p}>0.001$ (which is consistent with the observations in Fig 4.1) and the two solutions are found to converge for all practical purposes. Therefore, eq (4.34) is sufficient for predicting ductile damage growth unless small values of damage in the predominantly

\footnotetext{
${ }^{2}[46]$ vol 1 , Table 38 .

${ }^{3}$ The solutions of eqs (4.33) and (4.34) presented in this subsection were obtained numerically with the help of IMSL[55] Fortran subroutine DIVPRK, which solves an initial value problem using the RungeKutta-Verner fifth-order and sixth-order method.
} 
elastic range is of concern ${ }^{4}$. Fig $4.2(\mathrm{~b})$ shows damage growth in SAE 1035 steel as predicted by eq (4.34) and its three approximations (eqs $4.36,4.39,4.40$ - referred to as "closed-form", "approx soln" and "linear approx", respectively, in the figure). The material properties used for the predictions are from source (b) in Table 4.1. As shown in Fig. 4.2(b), predictions of damage growth by eqs (4.34) and (4.36) are practically indistinguishable with the plot of the latter lying just above the former. This was found to hold equally true for the other three materials studied, so only eq (4.34) was plotted in Figs 4.3 through 4.5. The other two approximations, though conservative, are not quite as close to the exact solution, and have not been plotted in the three remaining figures.

Fig. 4.3 compares the prediction of damage growth in 2024-T3 aluminum with experimental results from three different sources: (i) Chow and Wang (1987) on 2024-T3 $\mathrm{Al}$, (ii) Woo and $\mathrm{Li}$ (1993b) on the mean damage in 45 specimens of 2024-T3 Al, and (iii) Lemaitre (1985) on French aluminum alloy AU4G1 which is equivalent ${ }^{5}$ to $2024-\mathrm{T} 3$ A1. The material properties used in the present model are from source (d) in Table 4.1. Fig. 4.4 compares the prediction of damage growth in SAE 4130 steel with experimental results (Lemaitre, 1985) on French steel 30CD4 which is equivalent ${ }^{6}$ to SAE 4130 . The material properties used in the present model are from source (f2) in Table 4.1. Fig. 4.5 compares the prediction of damage growth (eq 4.34) in INCO 718 superalloy with experimental results (Lemaitre, 1985). The material properties used in the present model are from source (e3) in Table 4.1.

The proposed model (eq 4.34), it may be recalled, requires four mechanical properties: the elastic modulus $(E)$, the hardening modulus $(K)$, the hardening exponent $(M)$ and the true fracture $\operatorname{strength}^{7}\left(\sigma_{f}\right)$. In fact, only two parameters, $M$ and the ratio $\sigma_{f} / K$, are necessary if the simplified closed-form solution (eq 4.36) is used. In addition, eqs (4.34) and (4.36) require the threshold strain, $\epsilon_{0} \geq 0$, for the initial condition, which is not widely available. It will be shown in the following that ductile damage growth is quite insensitive to this parameter $\left(\epsilon_{0}\right)$, and in the absence of additional information, $\epsilon_{0}$ may be equated to zero, which would cause a slight error on the conservative side ${ }^{8}$.

In spite of the difficulties encountered in verifying the proposed model its excellent

\footnotetext{
${ }^{4}$ The proposed ductile damage growth model will be used in sec 4.3 .2 on creep, to determine the damage introduced by the initial loading which is treated as the initial damage for the creep damage growth equation (eq 4.55). Creep damage is rather sensitive to $D_{0}$ (even for small values of $D_{0}$ ), and eq (4.33) will be used instead of eq (4.34) to determine $D_{0}$ in creep, since small plastic strains may be involved.

${ }^{5}$ Aluminum and Aluminum Alloys, ASM, 1993, p. 653.

${ }^{6}[46]$ vol 1 , Table 39.

${ }^{7}$ It was recognized in sec 4.1 that the local failure stress, $\sigma_{c}$, may not be an easily available material property and its value was assumed to be close to the true fracture strength, $\sigma_{f}$. In fact, $\sigma_{c}$ could be located only for low carbon steel out of the four materials considered: $\sigma_{c}=1200 \mathrm{MPa}$ (for SAE 1045[82]) whereas $\sigma_{f}=707 M P a$ (for SAE 1008[9]), $\sigma_{f}=725 M P a$ (for SAE 1015 [34]), $\sigma_{f}=713 M P a$ (for AISI 1020 steel [34]). Substituting $\sigma_{f}$ for $\sigma_{c}$ gave very good results in Figures 4.3 through 4.5.

${ }^{8}$ As listed in Table $4.2, \epsilon_{0}$ is not much different from zero ( $2 \%$ or less) for structural materials when compared to the scales of fracture ductility, $\epsilon_{f}(20 \%$ to $100 \%$ or more).
} 
agreement with experimental results shows its stability with regard to minor variations in the material parameters. This suggests a way to build up a database of critical damage parameters for different materials and temperatures even in the absence of tests conducted for this specific purpose. The variability in damage growth, however, also points to something more important: the possibility of noise in the process which may be significant enough to affect the failure strain (or time) appreciably and thereby provides a motivation for a stochastic approach to damage growth.

\section{Sensitivity study}

The sensitivity of the proposed model (eq 4.34) to the five parameters $\left(E, K, M, \sigma_{f}\right.$, $\left.\epsilon_{0}\right)$ is shown in Fig. 4.6 through Fig. 4.10. The base values of the parameters chosen are: $E=200 \mathrm{GPa}, K=1000 \mathrm{MPa}, M=10, \sigma_{f}=1500 \mathrm{MPa}$ and $\epsilon_{0}=0$ which are typical of carbon steel.

Fig. 4.6 shows that at a given plastic strain level, a more rigid material suffers more damage. However, the model is quite insensitive to variations in $E$. Hence, in the stochastic formulation, to be presented in Chapter $5, E$ can be treated as a deterministic variable fixed at its mean value in order to save computational effort.

Fig 4.7 shows the damage predictions are reasonably sensitive to $K$. A two-fold increase (or decrease) in $K$ causes about a two-fold increase (or decrease) in damage for a given strain. That is, a material which undergoes more work hardening (higher $K$ ) for a given plastic strain, suffers more damage. In contrast, predicted damage is almost insensitive to values of $M$ above 10, as shown in Fig. 4.8. More ductile materials (those with higher $M$ ) start off suffering more damage for a given plastic strain, but if loaded long enough, their rate of damage accumulation decreases faster, and a more ductile material eventually suffers less damage.

Fig 4.9 shows that sensitivity to $\sigma_{f}$ is comparable to that to $K$, a two-fold increase (or decrease) causing almost a two-fold decrease (or increase) in damage. A stronger material (higher $\sigma_{f}$ ) suffers less damage at a given plastic strain. In other words, given the same critical damage, $D_{c}$, a material with lower true failure stress fails first. Moreover, with a higher damage threshold, a material predictably suffers less damage for a given plastic strain (fig. 4.10). However, the rate of damage growth increases slightly with increasing $\epsilon_{0}$.

No study could be located in the literature, experimental or otherwise, which determined the sensitivity of damage growth to one or more of the above parameters. The above predictions of sensitivity therefore remain to be verified by experimental observations. 


\subsection{Creep Damage}

\subsubsection{Damage Growth Predictions}

The principle of equivalence for elastic and plastic strains in sec 4.2 now is adopted for creep strain rate:

A damaged volume of material under the applied stress $\sigma$ shows the same creep strain rate at a given time and temperature as a comparable undamaged volume under the effective stress $\tilde{\sigma}$ at the same time and temperature.

Using the above principle, the uniaxial creep strain rate (eq 2.4), under constant applied stress $\sigma$ and at temperature $\theta$, can be written as:

$$
\dot{\epsilon}_{c r}= \begin{cases}A \phi \tilde{\sigma}^{m} t^{\phi-1} ; & \text { under effective stress } \\ \tilde{A} \phi \sigma^{m} t^{\phi-1} ; & \text { under nominal stress }\end{cases}
$$

where it is assumed that the exponents $m$ and $\phi$ are not affected by damage. Continuing with the assumption of isotropic damage and using eq (3.4), the relation between the damaged rate parameter $\tilde{A}$ and its undamaged value can be deduced from the above as

$$
\tilde{A}=\frac{A}{(1-D)^{m}}
$$

The material parameters are all temperature-dependent.

The total strain must be written in terms of three components:

$$
\epsilon=\epsilon_{e}+\epsilon_{p}+\epsilon_{c r}
$$

At constant stress creep, the elastic and plastic components of strain do not change. Hence, $d \epsilon=d \epsilon_{c r}$, and the free energy per unit volume under uniaxial monotonic loading reduces to

$$
\begin{aligned}
\psi & =\int_{\epsilon_{c 0}}^{\epsilon_{c r}} \sigma d \epsilon_{c}^{\prime}-\gamma \\
& =\sigma_{\infty}\left(\epsilon_{c r}-\epsilon_{c 0}\right)-\frac{3}{4} \sigma_{f}(\theta) D
\end{aligned}
$$

where $\sigma_{\infty}$ is the constant far-field applied stress (acting normal to the surface), and $\gamma$ is the surface energy of voids per unit volume of material. The temperature-dependence of the true failure stress, $\sigma_{f}$, has been emphasized in eq (4.47). The partial derivative of $\psi$ with respect to the isotropic damage variable then becomes

$$
\psi_{D}=-\frac{3}{4} \sigma_{f}(\theta)
$$


We now use eq (4.10) to obtain the differential equation for uniaxial constant stress creep damage growth under the assumption of isotropic damage:

$$
D^{\prime}=-\frac{\sigma_{\infty}}{\psi_{D}}
$$

where $D^{\prime}$ is the derivative with respect to the axial strain which, with creep as the dominant damaging mechanism, is equal to $d D / d \epsilon_{c r}$. The damage growth rate is:

$$
\frac{d D}{d \epsilon_{c r}}=\frac{\sigma_{\infty}}{\frac{3}{4} \sigma_{f}}
$$

Using eq (4.44), the time rate of damage growth can be written as

$$
\begin{aligned}
\frac{d D}{d t} & =\frac{4}{3} \frac{\sigma_{\infty}}{\sigma_{f}} \dot{\epsilon}_{c r} \\
& =\frac{4}{3} \frac{\sigma_{\infty}}{\sigma_{f}} \tilde{A} \sigma_{\infty}^{m} \phi t^{\phi-1}
\end{aligned}
$$

Substituting eq (4.45) into eq 4.51), we can write:

$$
\frac{d D}{d t}=\frac{4}{3} \frac{A \phi \sigma_{\infty}^{m+1}}{\sigma_{f}(1-D)^{m}} t^{\phi-1}
$$

For steady-state creep $(\phi=1)$, the damage growth rate simplifies to

$$
\frac{d D}{d t}=\frac{4}{3} \frac{A \sigma_{\infty}^{m+1}}{\sigma_{f}(1-D)^{m}}
$$

which can be written as:

$$
\frac{d D}{d t}=B\left(\frac{\sigma_{\infty}^{1+1 / m}}{1-D}\right)^{m}
$$

where $B=(4 / 3) A / \sigma_{f}$ is a temperature-dependent material constant. The above equation has almost the same form as Kachanov's kinetic equation of creep damage growth (eq 3.11) because the numerical value of $m$ (usually ranging between 4 and 12) renders the exponent of $\sigma_{\infty}$ sufficiently close to 1 . The present method therefore can derive from fundamental principles a long-established phenomenological model of creep damage growth and provide estimates of the phenomenological parameters.

Integrating eq (4.52), creep damage as a function of time is

$$
D(t)=1-\left[\left(1-D_{0}\right)^{m+1}-(4 / 3)\left(A / \sigma_{f}\right)(m+1) \sigma_{\infty}^{1+m} t^{\phi}\right]^{\frac{1}{1+m}}
$$


where the initiation time is taken to be $t_{0}=0$. Conversely, the time to failure ${ }^{9}$ in creep damage is:

$$
t_{f}=\left[\frac{\left(1-D_{0}\right)^{m+1}-\left(1-D_{c}\right)^{m+1}}{(4 / 3)\left(A / \sigma_{f}\right)(m+1) \sigma_{\infty}^{1+m}}\right]^{1 / \phi}
$$

Assuming an undamaged initial state $\left(D_{0}=0\right)$ and that $m$ is large enough to make $\left(1-D_{c}\right)^{m+1} \sim 0$ (even when $D_{c} \neq 1$ ), the time to failure can be simplified as

$$
t_{f}=\frac{1}{\left[(4 / 3)\left(A / \sigma_{f}\right)(m+1) \sigma_{\infty}^{1+m}\right]^{1 / \phi}}
$$

Damage growth may then also be written as a function of the normalized time, $t / t_{f}$ :

$$
D=1-\left[\left(1-D_{0}\right)^{m+1}-\left\{\left(1-D_{0}\right)^{m+1}-\left(1-D_{c}\right)^{m+1}\right\}\left(\frac{t}{t_{f}}\right)^{\phi}\right]^{\frac{1}{1+m}}
$$

Assuming an initially undamaged state $\left(D_{0}=0\right)$ in steady state creep $(\phi=1)$ and that $m$ is large enough (i.e., $\left(1-D_{c}\right)^{m+1} \sim 0$ ), we have

$$
D=1-\left[1-\frac{t}{t_{f}}\right]^{\frac{1}{1+m}}
$$

which is identical to the form proposed by Kachanov (1986).

The proposed model is now validated with available data on creep damage. Trends predicted by the proposed model with different loading conditions and material properties are also presented.

\subsubsection{Verification and Prediction of Trends}

Unlike ductile deformation damage, there exist (to the knowledge of the authors) published data on CDM-based creep damage growth for only one material and temperature: superalloy IN 100 at $1000^{\circ} \mathrm{C}$, the results being reproduced in Lemaitre $(1992)^{10}$ as well

\footnotetext{
${ }^{9}$ As discussed in sec. 3.2, failure is defined as the development of a macrocrack, or in absence of it, as rupture of the component.

${ }^{10}$ This reference contains two different figures of creep damage growth in IN 100 superalloy at $1000 \mathrm{C}$. It is however not clear whether they pertain to the same test: (i) $D$ is measured by the familiar CDM-based reduced modulus method $\left(D=1-\tilde{E} / E\right.$ ), which (along with $\epsilon_{c r}$ ) is plotted as a function of time, $t$. The critical damage is $\mathbf{0 . 2 2}$. The far-field stress level however is not reported. (ii) Creep damage is measured as $D(t)=1-\left[\dot{\epsilon}_{\min } / \dot{\epsilon}_{c}(t)\right]^{1 / m}$, and $D$ is reported in terms of the normalized time $t / t_{f}$. The relation of this damage variable to CDM is not obvious. The critical damage is 0.12 . The constant stress is $240 \mathrm{MPa}$ but the failure time, $t_{f}$, is not reported. Finally, it is not clear whether the reported ratio $t_{f} / t_{R}=0.9$ pertains to the first case, the second or to both.
} 
as in Chaboche (1988). Fig 4.11 plots the damage growth prediction (using eq 4.58 with $D_{0}=0, D_{c}=0.22, m=4$ and $\phi=1$ ) as a function of $t / t_{f}$ and compares it with the experimental results published in Lemaitre (1992). Since the Norton's law parameters for the specimen used are not reported along with the experimental results (Lemaitre, 1992), a representative value of $m=4$ is assumed in eq (4.58). Very good agreement with the experimental results is obtained.

Due to the paucity of CDM-based creep damage growth data, further validation of the proposed creep damage model is limited to how accurately it can predict (i) the time to failure, and (ii) trends in damage growth by varying the applied stress and temperature. ASTM A36 steel and Type 316 stainless steel are chosen for this purpose, due first to their importance in engineering applications and second to the availability of data on their high-temperature creep behaviour.

Tables 4.3 and 4.4 list creep law parameters (eq 2.4) and other mechanical properties for A36 steel and 316 stainless steel respectively at various temperatures. It should be noted that these parameters are applicable only within certain ranges of applied stresis for a given temperature, and these limits were adhered to as much as practicable while predicting damage growth in the following tables and figures.

Tables 4.5 and 4.6 list the predicted failure stresses at given values of time and temperature for ASTM A36 steel and compare them with experimental results ${ }^{11}$. The predictions in Table 4.5 are based on creep rate data from Fields and Fields (1989) and those in Table 4.6 are based on Harmathy (1967). The initial damage ( $D_{0}$ in eq 4.55) is assumed to be caused by the plastic deformation introduced when the specimen is loaded from zero to $\sigma_{\infty}$ at the beginning of the creep test. Its value is obtained from the monotonic ductile damage growth equation $(4.33)^{12}$. Two different $D_{c}$ values are chosen for eq (4.55), as the critical damage for A36 at the high temperatures is not known experimentally. However, due to the accelerated nature of creep damage growth, the failure condition is found to be rather insensitive to changes in $D_{c}$, and therefore the difficulty in determining the critical damage at different temperatures should not be an impediment to adopting a CDM model for creep. The quality of prediction in Table 4.6 based on the Fields and Fields (1989) data set becomes poorer with higher exposure times. This may be due to the fact that the Fields and Fields (1989) data are obtained from tests which lasted at most 16 hours. The validity of these parameters at exposures of $1,000,10,000$ and 100,000 hours are unknown.

Fig. 4.12 shows the effect of applied stress $\sigma_{\infty}$ on predicted creep damage growth in A36 steel as a function of time (eq 4.55) at a constant temperature, while Fig. 4.13 shows the effect of temperature, $\theta$, on predicted damage growth as a function of time (eq 4.55) at

\footnotetext{
${ }^{11}$ Two different sets of failure data were located in the literature $([12,13])$ for $A 36$ steel.

${ }^{12} \mathrm{Eq}(4.33)$ is solved numerically using the parameters $E, K, M, \sigma_{f}$ from Table 4.3 , and the value of damage corresponding to $\epsilon_{p}=\left(\sigma_{\infty} / K\right)^{M}$ is used as $D_{0}$ in eq (4.55). The value of $\sigma_{\infty}$ predicted to cause failure in a given exposure time and temperature is thus obtained in an iterative manner: first by obtaining $D_{0}$ with the help of eq (4.33), and then by obtaining $t_{f}$ with the help of eq (4.56) in each iteration.
} 
a constant applied stress. The initial damage arising out of the initial time-independent plastic straining has been incorporated in the above figures and tables. Besides plastic deformation, initial damage may also result from residual stresses, embrittlement etc., and an initial damage of only $10 \%$ can reduce the total creep life of a structure by as much as $50 \%$ (Fig 4.14).

Fig 4.15 shows the predicted growth of creep damage in 316 stainless steel at $1100^{\circ} \mathrm{F}$ $\left(593.3^{\circ} \mathrm{C}\right)$ for six different stress levels using data from Table 4.4. The initial damage is computed with the help of eq (4.33). The predicted times to failure (eq 4.56) are listed in Table 4.7 along with experimental results (Garofalo et al, 1961) ${ }^{13}$. Two different values of critical damage have been adopted, since $D_{c}$ is not known for type 316 stainless steel at $1100^{\circ} \mathrm{F}$. However, due to the accelerated nature of creep damage growth, the predicted $t_{f}$ is not especially sensitive to variability in $D_{c}$ if $D_{c}$ is high enough. For lower stresses, the predictions lie within the experimentally observed range of values, but the proposed model is found to over-predict the time to failure for higher stresses.

The existence of significant variabilities in the experimental failure stress at fixed $t_{f}$ and $\theta$, and in the experimental failure time at fixed $\sigma_{\infty}$ and $\theta$, is clearly seen from Tables 4.5 (or 4.6 ) and 4.7, respectively. This suggests the need of a stochastic analysis of creep damage growth.

\subsection{Fatigue Damage}

\subsubsection{Damage Analysis}

Fatigue failure occurs (after a sufficient number of cycles) at stress levels below the static monotonic failure stress, provided the stress amplitude exceeds the endurance limit. From the damage mechanics perspective, additional damage is introduced in the material volume with each cycle. Since accumulation of damage is an irreversible process, it does not disappear once the loading is reversed or withdrawn. Rather, the accumulated damage at the end of one cycle acts as the initial damage for the damage increment in the next cycle. This goes on until the critical value for damage is reached and failure is said to occur:

$$
\begin{aligned}
D_{i+1} & =D_{i}+\Delta D_{i}, \quad \Delta D_{i} \geq 0, \quad i=1, \ldots, N_{f}-1 \\
D_{N_{f}} & =D_{c}
\end{aligned}
$$

where $D_{i}$ is the damage at the end of the $i$ th stress cycle, $\Delta D_{i}$ is the damage increment during the $i$ th cycle, $D_{c}$ is the critical damage and $N_{f}$ is the cycles to failure. Failure, however, is not necessarily the occurrence of fracture. It is, as mentioned in section

\footnotetext{
${ }^{13}$ This reference lists the times to the end of primary and the secondary stages, $t_{1}$ and $t_{2}$ respectively, besides reporting the rupture time, $t_{R}$, for each stress level. Significant scatter can be seen in $t_{1}, t_{2}$ and $t_{R}$ at every stress level. The CDM-based $t_{f}$ is generally less than $t_{R}$ and is believed to lie somewhere between $t_{2}$ and $t_{R}$, but Garofalo et al (1961) did not list the time to the occurrences of the first macrocrack.
} 
3.2 , the limit where damage can no longer be said to be homogeneous or isotropic and the purview of damage mechanics ends. The CDM-based definition of damage ceases to be valid with the emergence of a dominant flaw - for example, the development of a macrocrack.

It is reasonable to assume that, in any given cycle, the unloading portion of a hysteresis loop and compressive stresses do not contribute to the damage increment. Consequently, only the reloading section above the endurance limit (if any) in the positive stress region causes damage to increase (similar restrictions about fatigue damage increment are also found in Kachanov, 1986; and Lemaitre, 1984). Under these assumptions, the basic mechanism of fatigue damage accumulation remains the same as that in monotonic loading and creep deformation. Thus, the same equation for isotropic damage growth (eq 4.9) may be written for the $i$ th stress cycle:

$$
\frac{d D}{d \epsilon}=\left\{\begin{array}{lll}
-\frac{\sigma_{\infty}}{\psi_{D}} & ; & \sigma_{\infty} \geq S_{e} \geq 0, \dot{\epsilon}>0 \\
0 & ; \quad \text { otherwise }
\end{array}\right.
$$

with the initial condition $D=D_{i-1}$.

The free energy (eq 4.12) must be computed differently from what was done in the case of monotonic loading, however. This is because the equation of the hysteresis loop is described with respect to the (cyclic) stress range-strain range coordinates $\Delta \epsilon$ and $\Delta \sigma$ (with origin at the loop tip) instead of in the original $\sigma-\epsilon$ coordinate system:

$$
\begin{aligned}
\Delta \epsilon & =\Delta \epsilon_{e}+\Delta \epsilon_{p} \\
\Delta \epsilon_{e} & =\frac{\Delta \tilde{\sigma}}{E} \\
\Delta \epsilon_{p} & =2\left(\frac{\Delta \tilde{\sigma}}{2 H}\right)^{M^{\prime}}
\end{aligned}
$$

in which the total strain range is the sum of its elastic and plastic constituents, $\Delta \tilde{\sigma}$ is the effective stress range, $E$ is the (undamaged) elastic modulus, $H$ is the (undamaged) cyclic hardening modulus and $M^{\prime}$ is the cyclic hardening exponent. The lower and the upper loop-tip coordinates in the $\epsilon-\sigma$ system are $\left(\epsilon_{\min }, \sigma_{\min }\right)$ and $\left(\epsilon_{\max }, \sigma_{\max }\right)$ respectively and they may vary from cycle to cycle. The relation between the new and the original coordinates is linear:

$$
\begin{aligned}
\Delta \epsilon & =\epsilon-\epsilon_{\min } \\
\Delta \sigma & =\sigma-\sigma_{\min }
\end{aligned}
$$

The effective stress range, $\Delta \tilde{\sigma}$, is

$$
\Delta \tilde{\sigma}=\tilde{\sigma}-\tilde{\sigma}_{\min }
$$


Using eq (3.4):

$$
\begin{aligned}
\Delta \tilde{\sigma} & =\frac{\sigma}{1-D}-\frac{\sigma_{\min }}{1-D} \\
& =\frac{\sigma-\sigma_{\min }}{1-D} \\
& =\frac{\Delta \sigma}{1-D}
\end{aligned}
$$

We now apply the principle of strain equivalence to the elastic and plastic strain ranges to obtain:

$$
\Delta \epsilon_{e}= \begin{cases}\frac{\Delta \tilde{\sigma}}{E} ; & \text { under effective stress range } \\ \frac{\Delta \sigma}{\tilde{E}} ; & \text { under nominal stress range }\end{cases}
$$

and

$$
\left(\Delta \epsilon_{p}\right)^{\frac{1}{M}}= \begin{cases}\frac{\Delta \tilde{\sigma}}{2^{1-1 / M^{\prime} H}} ; & \text { under effective stress range } \\ \frac{\sigma}{2^{1-1 / M^{\prime}} \tilde{H}} ; & \text { under nominal stress range }\end{cases}
$$

where $\tilde{E}$ and $\tilde{H}$ are the damaged moduli, and the cyclic strain hardening exponent, $M^{\prime}$, is assumed to be unaffected by damage.

The fact that fatigue damage does not occur when the applied stress is cycled below the endurance limit, $S_{e}$, suggests the existence of a threshold plastic strain-range of damage increment, $\Delta \epsilon_{0_{i}}$, analogous to the threshold plastic strain $\epsilon_{0}$ in the monotonic case. $\Delta \epsilon_{0_{i}}$ is cycle-dependent, as the effective stress-strain limits $\left(\tilde{\sigma}_{\min }, \epsilon_{\min }\right)$ may vary as cycling progresses. It can be estimated as

$$
\begin{aligned}
\Delta \epsilon_{0_{i}} & =2\left(\frac{S_{e}-\tilde{\sigma}_{\min }}{2 H}\right)^{M^{\prime}} \\
& =2\left(\frac{\left(1-D_{i-1}\right) S_{e}-\sigma_{\min }}{2 \tilde{H}}\right)^{M^{\prime}}
\end{aligned}
$$

Using the relation between effective stress range and nominal stress range (eq 4.69), we obtain

$$
\left.\begin{array}{rl}
\tilde{E} & =E(1-D) \\
\tilde{H} & =H(1-D)
\end{array}\right\} \text { for } \Delta \epsilon>\Delta \epsilon_{0_{i}}
$$

The values of the stiffness moduli remain unchanged in cycle $i$ until the strain-range reaches the threshold value for that cycle:

$$
\left.\begin{array}{rl}
\tilde{E} & =E\left(1-D_{i-1}\right) \\
\tilde{H} & =H\left(1-D_{i-1}\right)
\end{array}\right\} \text { for } \Delta \epsilon \leq \Delta \epsilon_{0_{i}}
$$


The stress range expressed in terms of the plastic strain range is

$$
\Delta \sigma=2^{1-1 / M^{\prime}} \tilde{H}\left(\Delta \epsilon_{p}\right)^{1 / M^{\prime}}
$$

The reloading curve cuts the strain-axis at $\Delta \epsilon=\Delta \epsilon_{1}$, corresponding to which the plastic strain range is $\Delta \epsilon_{p}=\Delta \epsilon_{p 1}$. The intercept $\sigma_{\min }$ can be expressed as

$$
\sigma_{\min }=-2^{1-1 / M^{\prime}} \tilde{H}\left(\Delta \epsilon_{p 1}\right)^{1 / M^{\prime}}
$$

Equations (4.75) and (4.76) will be used subsequently to obtain the partial derivative of: the free energy and in the equation of damage growth.

Since the limit $\epsilon_{\min }$ does not change in a given cycle, we have

$$
d \Delta \epsilon=d \epsilon
$$

for that cycle. This allows us to write eq (4.62) for the $i$ th cycle as

$$
\frac{d D}{d \epsilon}=\frac{d D}{d \Delta \epsilon}=\left\{\begin{array}{lll}
-\frac{\Delta \sigma_{\infty}+\sigma_{\min }}{\psi_{D}(\Delta \epsilon)} & ; & \Delta \sigma_{\infty}+\sigma_{\min } \geq S_{e} \geq 0, \dot{\epsilon}>0 \\
0 & ; & \text { otherwise }
\end{array}\right.
$$

The free energy per unit volume for the $i$ th cycle can be written in terms of the loop-tip coordinates as

$$
\begin{aligned}
\psi & =\int_{\epsilon_{0_{i}}}^{\epsilon} \sigma d \epsilon^{\prime}-\frac{3}{4} \sigma_{f}\left(D-D_{i-1}\right) \\
& =\int_{\Delta \epsilon_{0_{i}}}^{\Delta \epsilon}\left(\Delta \sigma+\sigma_{\min _{i}}\right) d \Delta \epsilon^{\prime}-\frac{3}{4} \sigma_{f}\left(D-D_{i-1}\right)
\end{aligned}
$$

which allows the partial derivative $\psi_{D}$ to be expressed in terms of the strain range coordinates as:

$$
\begin{aligned}
\psi_{D}(\Delta \epsilon) & =\int_{\Delta \epsilon_{0_{i}}}^{\Delta \epsilon} \frac{\partial}{\partial D}(\Delta \sigma) d \Delta \epsilon^{\prime}+\int_{\Delta \epsilon_{0_{i}}}^{\Delta \epsilon} \frac{\partial}{\partial D}\left(\sigma_{\text {min }_{i}}\right) d \Delta \epsilon^{\prime}-\frac{3}{4} \sigma_{f} \\
& =\int_{\Delta \epsilon_{0_{i}}}^{\Delta \epsilon} \frac{\partial}{\partial D}(\Delta \sigma) d \Delta \epsilon^{\prime}+\frac{\partial}{\partial D}\left(\sigma_{\min }\right) \int_{\Delta \epsilon_{0_{i}}}^{\Delta \epsilon} d \Delta \epsilon^{\prime}-\frac{3}{4} \sigma_{f}
\end{aligned}
$$

Decomposing the total strain range, using the principle of strain equivalence for the elastic and plastic strain ranges, and eqs (4.74) - (4.76), the partial derivative of the free energy per unit volume in the $i$ th cycle is found to be:

$$
\begin{aligned}
\psi_{D}\left(\Delta \epsilon_{p}\right)= & -\frac{K^{\prime 2}}{2 E}\left[\Delta \epsilon_{p}^{2 / M^{\prime}}-\Delta \epsilon_{0_{i}}^{2 / M^{\prime}}\right]-\frac{K^{\prime}}{1+\frac{1}{M^{\prime}}}\left[\Delta \epsilon_{p}^{1+1 / M^{\prime}}-\epsilon_{p 0_{i}}^{1+1 / M^{\prime}}\right] \\
& +\frac{K^{\prime 2}}{E} \Delta \epsilon_{p 1_{i}}^{1 / M^{\prime}}\left(\Delta \epsilon_{p}^{1 / M^{\prime}}-\Delta \epsilon_{0_{i}}^{1 / M^{\prime}}\right)+K^{\prime} \Delta \epsilon_{p 1_{i}}^{1 / M^{\prime}}\left(\Delta \epsilon_{p}-\Delta \epsilon_{0_{i}}\right) \\
& -\frac{3}{4} \sigma_{f}
\end{aligned}
$$


where $K^{\prime}=2^{1-1 / M^{\prime}} H$. The differential equation of damage growth in cycle $i$ is then

$$
\begin{aligned}
\frac{d D}{1-D}= & \frac{\left\{K^{\prime}\left(\Delta \epsilon_{p}\right)^{1 / M^{\prime}}-K^{\prime}\left(\Delta \epsilon_{p 1_{i}}\right)^{1 / M^{\prime}}\right\} d \Delta \epsilon_{p}}{\left[\frac{K^{\prime 2}}{2 E}\left\{\Delta \epsilon_{p}^{2 / M^{\prime}}-\Delta \epsilon_{0_{i}}^{2 / M^{\prime}}\right\}+\frac{K^{\prime}}{1+\frac{1}{M^{\prime}}}\left\{\Delta \epsilon_{p}^{1+1 / M^{\prime}}-\epsilon_{p 0_{i}}^{1+1 / M^{\prime}}\right\}\right.} \\
& \left.-\frac{K^{\prime 2}}{E} \Delta \epsilon_{p 1_{i}}^{1 / M^{\prime}}\left(\Delta \epsilon_{p}^{1 / M^{\prime}}-\Delta \epsilon_{0_{i}}^{1 / M^{\prime}}\right)-K^{\prime} \Delta \epsilon_{p 1_{i}}^{1 / M^{\prime}}\left(\Delta \epsilon_{p}-\Delta \epsilon_{0_{i}}\right)+\frac{3}{4} \sigma_{f}\right]
\end{aligned}
$$

with the initial condition $D=D_{i-1}$ at $\Delta \epsilon_{p}=\Delta \epsilon_{0_{i}}$. The value $D_{i}$, the damage at the end of cycle $i$, is the solution of the above differential equation at $\Delta \epsilon_{p}=\Delta \epsilon_{p m_{i}}$, which is the maximum plastic strain range for that cycle.

For engineering metals, the ratio $H / E \sim 0$ (i.e., $K^{\prime} / E \sim 0$ ), allowing us to simplify eq (4.82) to:

$$
\frac{d D}{1-D}=\frac{\left(\Delta \epsilon_{p}\right)^{1 / M^{\prime}}-\left(\Delta \epsilon_{p 1_{i}}\right)^{1 / M^{\prime}}}{\frac{1}{1+\frac{1}{M^{\prime}}}\left[\Delta \epsilon_{p}^{1+1 / M^{\prime}}-\epsilon_{p 0_{i}}^{1+1 / M^{\prime}}\right]-\Delta \epsilon_{p 1_{i}}^{1 / M^{\prime}}\left(\Delta \epsilon_{p}-\Delta \epsilon_{0_{i}}\right)+\frac{3}{4} \sigma_{f} / K^{\prime}} d \Delta \epsilon_{p}
$$

which has the closed-form solution:

$$
-\log (1-D)=\log \left[\frac{1}{1+\frac{1}{M^{\prime}}}\left(\Delta \epsilon_{p}^{1+1 / M^{\prime}}-\epsilon_{p 0_{i}}^{1+1 / M^{\prime}}\right)-\Delta \epsilon_{p 1_{i}}^{1 / M^{\prime}}\left(\Delta \epsilon_{p}-\Delta \epsilon_{0_{i}}\right)+\frac{3}{4} \frac{\sigma_{f}}{K^{\prime}}\right]+C_{0}
$$

$C_{0}$ being the constant of integration. Using the initial condition $D=D_{i-1}$ at $\Delta \epsilon_{p}=\Delta \epsilon_{0_{i}}$ as stated above, the damage at the end of cycle $i$ is

$$
D_{i}=1-\left(1-D_{i-1}\right) \frac{\frac{1}{1+\frac{1}{M^{\prime}}} \Delta \epsilon_{0_{i}}^{1+1 / M^{\prime}}-\Delta \epsilon_{p 1_{i}}^{1 / M^{\prime}} \Delta \epsilon_{0_{i}}+C_{i}}{\frac{1}{1+\frac{1}{M^{\prime}}} \Delta \epsilon_{p m_{i}}^{1+1 / M^{\prime}}-\Delta \epsilon_{p 1_{i}}^{1 / M^{\prime}} \Delta \epsilon_{p m_{i}}+C_{i}}
$$

where

$$
C_{i}=\frac{3}{4} \frac{\sigma_{f}}{K^{\prime}}-\frac{1}{1+\frac{1}{M^{\prime}}} \Delta \epsilon_{0_{i}}^{1+1 / M^{\prime}}+\Delta \epsilon_{p 1_{i}}^{1 / M^{\prime}} \Delta \epsilon_{0_{i}}
$$

and $\Delta \epsilon_{p m_{i}}$ is the maximum plastic strain range in cycle $i$, which is attained just prior to the start of unloading in the hysteresis loop. The recursive nature of eq (4.85) can be used to express damage at the end of $n$ cycles in terms of the initial damage, $D_{0}$ :

$$
D_{n}=1-\left(1-D_{0}\right) \prod_{i=1}^{n} \frac{\frac{1}{1+\frac{1}{M^{\prime}}} \Delta \epsilon_{0_{i}}^{1+1 / M^{\prime}}-\Delta \epsilon_{p 1_{i}}^{1 / M^{\prime}} \Delta \epsilon_{0_{i}}+C_{i}}{\frac{1}{1+\frac{1}{M^{\prime}}} \Delta \epsilon_{p m_{i}}^{1+1 / M^{\prime}}-\Delta \epsilon_{p 1_{i}}^{1 / M^{\prime}} \Delta \epsilon_{p m_{i}}+C_{i}}
$$

In the case of strain-controlled loading, the strain ranges $\Delta \epsilon_{m i}, \Delta \epsilon_{p m_{i}}, \Delta \epsilon_{p 1 i}$ and the parameter $C_{i}$ are independent of $i$, giving the following simplification:

$$
D_{n}=1-\left(1-D_{0}\right)\left(\frac{\frac{1}{1+\frac{1}{M^{\prime}}} \Delta \epsilon_{p D}^{1+1 / M^{\prime}}-\Delta \epsilon_{p 1}^{1 / M^{\prime}} \Delta \epsilon_{p D}+C}{\frac{1}{1+\frac{1}{M^{\prime}}} \Delta \epsilon_{p}^{1+1 / M^{\prime}}-\Delta \epsilon_{p 1}^{1 / M^{\prime}} \Delta \epsilon_{p}+C}\right)^{n}
$$


It may be recalled from the discussion in sec. 3.2 that CDM-based predictions of fatigue damage growth are confined to the initiation phase of fatigue life ${ }^{14}$. The propagation phase can be effectively dealt with the help of fracture mechanics. Eqs (4.82) - (4.88) are capable of predicting the crack initiation life, $N_{I}$, using widely available materia]. parameters like $E, H, M^{\prime}, \sigma_{f}$ and $S_{e}$. It should be noted that no undetermined materialdependent constant has been introduced in the present formulation. In the following subsection, damage growth predictions for different materials and loading conditions are: compared with experimental data obtained from various sources. Since available data on initiation life are limited (due partly to the non-unique definition of initiation, and partly to the difficulty in detecting small cracks), some $N_{I}$ predictions are supplemented with computed $N_{P}$ values and then compared with the more easily available data on the total number of cycles to failure.

\subsubsection{Verification of the Proposed Model}

The material properties and their sources used in the following damage growth predictions are listed in Table 4.8 .

Fig 4.16 predicts damage growth (using eq 4.88) in strain-controlled fatigue tests of AISI 316 stainless steel with $\Delta \epsilon_{p}=0.81 \%$ and $D_{c}=0.15$. The prediction is presented in terms of normalized cycles $N / N_{T}$ in order to compare with Chaboche $(1988)^{15}$. The agreement with experimental results is not good in Fig 4.16 which may be due in part to using material parameters in the model different from those of the test specimens.

Fig. 4.17 shows strain-controlled fatigue test results for $\mathrm{A} 106$-Grade $\mathrm{B}$ steel at $288^{\circ} \mathrm{C}$ in air. Predictions using eq (4.88) are compared with three sets of test data: (i) Number of cycles to crack initiation (Majumdar et al, 1993), (ii) number of cycles to a $25 \%$ drop in the peak tensile stress (Keisler et al, 1995) and (iii) number of cycles to failure (Majumdar et al, 1993). Here, predicted values of $N_{I}$ are seen to match experimental results quite well. The present model is also seen to project a general trend in fatigue behavior (eq 2.11): at low-cycle fatigue, the crack initiation period is negligible and most of the fatigue life is spent in crack propagation; while in high cycle fatigue, most of the life is taken up by crack initiation. The present model therefore may be said to predict the first component of the strain-life equation (2.11).

Fig. 4.18 compares (i) the predicted $N_{I}$ curve (using eq 4.88$)^{16}$ with the number of

\footnotetext{
${ }^{14}$ Discussion on the different phases of fatigue life is presented in sec. 2.3 .2

${ }^{15}$ The material properties of the specimen used were not reported in Chaboche (1988), nor were the number of cycles to failure. However, Lemaitre (1992) reports critical damage $D_{c}=0.15$ for the same nominal grade of material. This value of $D_{c}$ was utilized to estimate $N_{I}=0.87 N_{T}$ from Chaboche (1988). The remaining properties required in eq (4.88) were obtained from other sources for the same nominal grade of steel, as listed in table 4.8 .

${ }^{16}$ Since experimentally determined $D_{c}$ for quenched and tempered SAE 4340 is not available, it was determined analytically as $D_{c}=0.46$ (corresponding to $\epsilon_{f}=0.83$ ) with the help of the proposed ductile damage model (eq 4.34).
} 
cycles to the development of a crack of length $0.038 \mathrm{~mm}$ in SAE 4340 steel (Dowling, 1993) ${ }^{17}$ and (ii) the predicted $N_{T}$ curve with the number of cycles to failure (Topper and Morrow, 1970) in fully reversed strain-controlled fatigue cycling of quenched and tempered SAE 4340 steel $^{18}$. The predicted $N_{T}=N_{I}+N_{P}$ curve is obtained by summing $N_{I}$ (as obtained before) with the crack propagation life, $N_{P}$, obtained with the help of the Paris-Erdogan Law (eq 2.14 with $\Delta K_{t h}=10 M P a \sqrt{ } \mathrm{m}$ ) in the presence of crack-tip plasticity, as described in Appendix A. Both the predicted $N_{I}$ and $N_{T}$ curves are seen to match the experimental data quite well.

\subsubsection{Prediction of Load Sequencing Effects}

The load sequencing effect in fatigue cycling (described in sec 2.5) is a challenging area in modeling fatigue damage growth. Its study is especially important since variable amplitude loading is common in practice but almost all fatigue data pertain to fixedlimit load cycling. A state-of-the-art review of the subject (Schutz, 1993) makes it clear that all the existing rules for predicting damage under variable amplitude loading are far from perfect. In view of its practical importance and the shortcoming of existing methods, the problem of load sequencing is treated in a separate subsection here.

The proposed model of fatigue damage growth predicts the crack initiation life, $N_{I}$, in a recursive manner. It can easily incorporate variable amplitude stress (or strain) cycling, and predict the number of cycles to a macro-crack initiation. The present research however was unable to locate any published data that indicated how the crack initiation life was affected by load sequencing effects, though a sizable set of results are available when the propagation life is included (e.g., Manson et al, 1967; Wheeler, 1972; Miller, 1977). The ability of the present method to portray load sequencing effects therefore is limited to predicting the general trends.

In the absence of experimental data, let us confine the variable load to just two levels: $\underline{S}^{1}$ for $n_{1}$ cycles and $\underline{S}^{2}$ for $n_{2}$ cycles. The load level $\underline{S}^{1}$ represents two fixed limits of applied stress (or strain) cycling as does $\underline{S}^{2}$. Eq (4.87), which predicts damage after $n$ cycles, may be abbreviated as

$$
D_{n}=1-\left(1-D_{0}\right) \prod_{i=1}^{n} f\left(\underline{\epsilon}_{i} ; \Omega\right)
$$

where $\underline{\epsilon}$ represents the strain limits in cycle $i$, and $\Omega$ is the set of material parameters. When $\underline{S}^{1}$ is applied first,

$$
D_{n_{1}}=1-\left(1-D_{0}\right) \prod_{i=1}^{n_{1}} f\left(\underline{\epsilon}_{i}^{1} ; \Omega\right)
$$

\footnotetext{
${ }^{17}$ It should be noted that the steel in Dowling (1993), though of the same nominal grade, has different material properties (like $S_{u}$ and $f_{y}$ ) from the one in Endo and Morrow (1969) probably because it was not heat treated.

${ }^{18}$ The material data used (listed in Table 4.8) are taken from Endo and Morrow (1969), and Topper and Morrow (1970) refer to the same set of tests.
} 
After $\underline{S}^{2}$ is applied subsequently, the total damage is

$$
\begin{aligned}
D_{n_{1}, n_{2}} & =1-\left(1-D_{n_{1}}\right) \prod_{i=n_{1}+1}^{n_{1}+n_{2}} f\left(\underline{\epsilon}_{i}^{2} ; \Omega\right) \\
& =1-\left(1-D_{0}\right) \prod_{i=1}^{n_{1}} f\left(\underline{\epsilon}_{i}^{1} ; \Omega\right) \prod_{i=n_{1}+1}^{n_{1}+n_{2}} f\left(\underline{\epsilon}_{i}^{2} ; \Omega\right)
\end{aligned}
$$

Conversely, if $\underline{S}^{2}$ is applied first,

$$
D_{n_{2}}=1-\left(1-D_{0}\right) \prod_{i=1}^{n_{2}} f\left(\underline{\epsilon}_{i}^{2} ; \Omega\right)
$$

After $\underline{S}^{1}$ is applied subsequently, the total damage is:

$$
\begin{aligned}
D_{n_{2}, n_{1}} & =1-\left(1-D_{n_{2}}\right) \prod_{i=n_{2}+1}^{n_{1}+n_{2}} f\left(\underline{\epsilon}_{i}^{1} ; \Omega\right) \\
& =1-\left(1-D_{0}\right) \prod_{i=1}^{n_{2}} f\left(\underline{\epsilon}_{i}^{2} ; \Omega\right) \prod_{i=n_{2}+1}^{n_{1}+n_{2}} f\left(\underline{\epsilon}_{i}^{1} ; \Omega\right)
\end{aligned}
$$

It is obvious that $D_{n_{1}, n_{2}}$ in general is different from $D_{n_{2}, n_{1}}$. We can, however, find a condition that would make these two equal and a Miner type cumulative fatigue damage rule valid: Consider,

$$
\begin{array}{lll}
\underline{\epsilon}_{i}^{1}=\underline{\epsilon}^{1}, & \forall i \epsilon\left[1, n_{1}+n_{2}\right] \\
\underline{\epsilon}_{i}^{2}=\underline{\epsilon}^{2}, & \forall i \in\left[1, n_{1}+n_{2}\right]
\end{array}
$$

which means the strain limits in every cycle are independent of the past. Under this condition,

$$
\begin{aligned}
D_{n_{1}, n_{2}} & =1-\left(1-D_{0}\right) \prod_{i=1}^{n_{1}} f\left(\underline{\epsilon}^{1} ; \Omega\right) \prod_{i=n_{1}+1}^{n_{1}+n_{2}} f\left(\underline{\epsilon}^{2} ; \Omega\right) \\
& =1-\left(1-D_{0}\right) f^{n_{1}}\left(\underline{\epsilon}^{1} ; \Omega\right) f^{n_{2}}\left(\underline{\epsilon}^{2} ; \Omega\right)
\end{aligned}
$$

and

$$
\begin{aligned}
D_{n_{2}, n_{1}} & =1-\left(1-D_{0}\right) \prod_{i=1}^{n_{2}} f\left(\underline{\epsilon}^{2} ; \Omega\right) \prod_{i=n_{2}+1}^{n_{1}+n_{2}} f\left(\underline{\epsilon}^{1} ; \Omega\right) \\
& =1-\left(1-D_{0}\right) f^{n_{2}}\left(\underline{\epsilon}^{2} ; \Omega\right) f^{n_{1}}\left(\underline{\epsilon}^{1} ; \Omega\right)
\end{aligned}
$$


and the two accumulated damages are equal. This means that if no strain hardening or softening occurs, and if the cycling takes place between exactly the same strain limits for a given load level (irrespective of where in the life of the component this load is applied), then the load sequencing effect vanishes. Of course this is an unrealistic situation in practice, and accordingly, Miner's rule is only an approximation.

Fig 4.19 shows two different damage growth trajectories in SAE 4340 steel: one for a high-low sequence and the other for a low-high sequence; the duration of the individual blocks is the same in both cases. The material properties are taken from Table 4.8 . The damage caused by the high-low sequence after 120 cycles is about 0.9 , while the damage caused by the low-high sequence after the same number of cycles is about 0.25 , thus demonstrating an observed trend in load-sequencing effect (e.g., Kutt and Bieniek, 1988).

Fig 4.20 shows the number of cycles to failure when the number of cycles at each level is variable. The total life is greater if the lower stress is applied first for the same number of cycles. The Miner's rule however, plots as a straight line and cannot distinguish between the ordering of the blocks.

\subsection{Closure}

An approach to damage prediction was established from first principles of thermodynamics, making the assumptions that there is zero temperature gradient, damage is isotropic and progresses close to equilibrium, and the force-displacement relation is linear at the micro-scale. The resulting equations of damage growth for ductile deformation, creep and fatigue generally show reasonably good agreement with experimental results by other authors on various ferrous and non-ferrous alloys. No undetermined constants have been introduced; rather, only common material parameters are included in the equations.

Damage mechanics can model the crack initiation stage of fatigue life and may be said to represent the aggregate behaviour of numerous short cracks prior to the appearance of any long crack. However, no widely accepted non-empirical method has existed thus far which could predict crack initiation in a defect-free (un-notched and polished) specimen. Together, damage mechanics and fracture mechanics can predict the entire fatigue life of a structure and complement one another.

The damage predictions are in qualitative agreement with the existing phenomenological/empirical models - e.g., the creep damage model of Kachanov (1986), the arbitrary dissipation potential model of Lemaitre (1985) for ductile flow, and the Basquin model for fatigue - thereby showing that analytical models can replace phenomenological/empirical ones. The present model can also account for load sequencing effects in fatigue cycling.

It has also been demonstrated that the critical damage is almost always less than 1 
and is different for different materials at a given temperature. This lends credence to the idea that $D_{c}$ is material property (discussed in sec 3.2 ); however, extensive testing and documentation are required to verify this claim. The attainment of critical damage corresponds to the formation of a macroscopic crack. The assumptions underlying CDM clearly break down once this happens. Hence, damage growth laws and damage predictions obtained from CDM-based approaches are limited to microscopic crack growth and crack nucleation prior to the development of a dominant crack.

The approach to damage growth in this chapter was deterministic (i.e., non-random). But the possibility of randomness in damage growth was apparent throughout - in the form of variabilities in material parameters and failure times. It will be shown in the next chapter that damage growth is indeed a random process, and the present model will be extended to include material variabilities as well as noise in the damage growth process. 


\begin{tabular}{|c|c|c|c|c|c|c|c|}
\hline Material & $\begin{array}{l}\text { Treatment } \\
\& \text { condition }\end{array}$ & Form & $\begin{array}{l}\mathrm{E} \\
\mathrm{GPa}\end{array}$ & $\begin{array}{l}\mathrm{K} \\
\mathrm{MPa}\end{array}$ & $\mathbf{M}$ & $\begin{array}{l}\sigma_{f} \\
\mathrm{MPa}\end{array}$ & $\epsilon_{f}$ \\
\hline $\mathrm{SAE} 1035^{b}$ & $\begin{array}{l}\text { austenitized } \\
\& \text { quenched }\end{array}$ & $\begin{array}{l}\text { round } \\
\text { tensile }\end{array}$ & 180 & 871 & 3.4 & 1200 & 1.03 \\
\hline AISI $1008^{e 1}$ & $\mathrm{x}$ & $\mathbf{x}$ & 200 & 661 & 4.3 & 707 & 1.61 \\
\hline $2024 \mathrm{Al}^{c 1}$ & $\mathrm{~T} 3$ & $\mathrm{x}$ & 73 & $\mathrm{x}$ & $\mathbf{x}$ & $\mathrm{x}$ & 0.18 \\
\hline $2024 \mathrm{Al}^{d}$ & T3 & $\mathrm{x}$ & 74.5 & 680 & 5.5 & 435 & 0.18 \\
\hline $2024 \mathrm{Al}^{f 1}$ & $\mathrm{~T} 3$ & $\mathrm{x}$ & 73.3 & 505 & 23.8 & $\mathbf{x}$ & $\mathrm{x}$ \\
\hline SAE $4130^{a}$ & annealed & sheet & $\mathrm{x}$ & 1167 & 8.47 & $\mathbf{x}$ & $\mathrm{x}$ \\
\hline $\operatorname{SAE} 4130^{a}$ & $\begin{array}{l}\text { normalized \& } \\
\text { temper-rolled }\end{array}$ & sheet & $\mathrm{x}$ & 1065 & 6.41 & $\mathrm{x}$ & $\mathrm{x}$ \\
\hline AISI $4130^{f 2}$ & $\begin{array}{l}\text { tempered \& } \\
\text { quenched }\end{array}$ & $\begin{array}{l}\text { hour } \\
\text { glass }\end{array}$ & 221 & 1117 & 15.9 & 1692 & 1.12 \\
\hline INCO $718^{c 2}$ & $\begin{array}{l}\text { precipitation } \\
\text { hardened }\end{array}$ & $\mathrm{x}$ & 211 & $\mathbf{x}$ & $\mathrm{x}$ & $\mathrm{x}$ & 0.12 \\
\hline INCO $718^{e 3}$ & heat-treated & sheet & 207 & 1435 & 23.8 & $1312^{*}$ & $\mathbf{x}$ \\
\hline
\end{tabular}

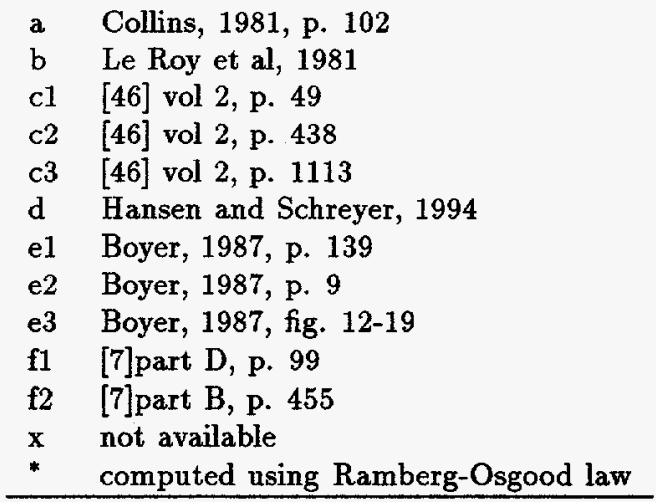

Table 4.1: Room temperature monotonic material properties of various alloys 


\begin{tabular}{|c|c|c|c|c|c|}
\hline \multirow[t]{2}{*}{ material } & \multicolumn{4}{|c|}{ experimental } & \multirow{2}{*}{$\begin{array}{c}\text { predicted }^{a} \\
D_{c}\end{array}$} \\
\hline & reference & $\epsilon_{0}$ & $\epsilon_{f}$ & $D_{c}$ & \\
\hline $\begin{array}{l}\text { SAE } 1035 \text { steel } \\
\text { (AFNOR XC 38) }\end{array}$ & Lemaitre, 1985 & 0 & 0.56 & 0.22 & 0.26 \\
\hline 2024-T3 Al & $\begin{array}{l}\text { Lemaitre, } 1985 \\
\text { Chow \& Wei, } 1987 \\
\text { Woo \& Li, } 1991\end{array}$ & $\begin{array}{l}0.02 \\
0 \\
0.0084^{b}\end{array}$ & $\begin{array}{l}0.25 \\
0.32 \\
0.25^{c}\end{array}$ & $\begin{array}{l}0.23 \\
0.22 \\
0.22^{b}\end{array}$ & $\begin{array}{l}0.25 \\
0.29 \\
0.25\end{array}$ \\
\hline SAE 4130 steel & Lemaitre, 1985 & 0.02 & 0.37 & 0.24 & 0.22 \\
\hline INCO 718 & Lemaitre, 1985 & 0.02 & 0.29 & 0.24 & 0.26 \\
\hline
\end{tabular}

${ }^{a}$ Solution of eq (4.34) for $D$ at $\epsilon_{p}=\epsilon_{f}$ using material properties listed in Table 4.1

${ }^{b}$ mean values from 45 specimens

${ }^{c}$ Maximum tabulated strain value

Table 4.2: Experimental and predicted critical damage in ductile deformation 


\begin{tabular}{|c|c|c|c|c|c|c|c|c|c|c|c|c|c|}
\hline \multirow{2}{*}{\multicolumn{2}{|c|}{$\theta$}} & \multicolumn{4}{|c|}{ Fields \& Fields, $1989^{a}$} & \multicolumn{3}{|c|}{ Harmathy, $1967^{b}$} & \multirow[b]{2}{*}{$\sigma_{u}^{d}$} & \multirow[b]{2}{*}{$\begin{array}{l}\sigma_{f}^{e} \\
\mathrm{ksi}\end{array}$} & \multirow[b]{2}{*}{$\begin{array}{c}E^{f} \\
10^{3} \mathrm{ksi}\end{array}$} & \multirow[b]{2}{*}{$\begin{array}{l}K^{f} \\
\mathrm{ksi}\end{array}$} & \multirow[b]{2}{*}{$M^{f}$} \\
\hline & & $\begin{array}{c}A \\
\text { ksi, hr }\end{array}$ & $m$ & $\phi$ & $\begin{array}{c}\text { test }^{c} \sigma \\
\text { ksi }\end{array}$ & $\begin{array}{c}A \\
\text { ksi, hr }\end{array}$ & $m$ & $\bar{\phi}$ & & & & & \\
\hline 350 & 662 & $1.69 \times 10^{-10}$ & 3.8 & 0.15 & $32-61$ & $\mathrm{x}$ & $\mathrm{x}$ & $\mathrm{x}$ & 1.06 & 106 & 24.9 & 43.15 & 3.92 \\
\hline 400 & 752 & $1.18 \times 10^{-10}$ & 3.9 & 0.31 & $21-56$ & $2.70 \times 10^{-13}$ & 4.7 & 1 & 0.95 & 95 & 24.3 & 42.41 & 4.82 \\
\hline 427 & 800 & $1.16 \times 10^{-10}$ & 4.4 & 0.39 & $21-56$ & $2.44 \times 10^{-12}$ & 4.7 & 1 & 0.83 & 83 & 23.9 & 40.67 & 5.49 \\
\hline 450 & 842 & $1.15 \times 10^{-10}$ & 4.9 & 0.45 & $21-48$ & $1.46 \times 10^{-12}$ & 4.7 & 1 & 0.72 & 72 & 23.6 & 39.18 & 6.06 \\
\hline 482 & 900 & $1.28 \times 10^{-10}$ & 6.4 & 0.53 & $21-48$ & $1.45 \times 10^{-10}$ & 4.7 & 1 & 0.67 & 67 & 23.2 & 35.58 & 6.86 \\
\hline 500 & 932 & $1.35 \times 10^{-10}$ & 7.2 & 0.58 & $21-37$ & $4.74 \times 10^{-10}$ & 4.7 & 1 & 0.64 & 64 & 23.0 & 33.55 & 7.32 \\
\hline 538 & 1000 & $3.74 \times 10^{-10}$ & 6.2 & 0.68 & $16-37$ & $4.93 \times 10^{-9}$ & 4.7 & 1 & 0.54 & 54 & 22.5 & 26.50 & 9.13 \\
\hline 550 & 1022 & $4.49 \times 10^{-10}$ & 5.9 & 0.71 & $16-27$ & $1.00 \times 10^{-8}$ & 4.7 & 1 & 0.51 & 51 & 22.4 & 24.27 & 9.70 \\
\hline 600 & 1112 & $3.79 \times 10^{-9}$ & 6.5 & 1.00 & $10-18$ & $1.50 \times 10^{-7}$ & 4.7 & 1 & 0.34 & 34 & 21.7 & 16.20 & 11.40 \\
\hline 650 & 1202 & $4.03 \times 10^{-8}$ & 4.7 & 1.24 & $5-11$ & $1.67 \times 10^{-6}$ & 4.7 & 1 & 0.23 & 23 & 21.1 & 10.96 & 17.00 \\
\hline 704 & 1300 & $\mathbf{x}$ & $\mathrm{x}$ & $\mathrm{x}$ & $\mathbf{x}$ & $1.75 \times 10^{-5}$ & 4.7 & 1 & 0.19 & 19 & $\mathrm{x}$ & $\mathrm{x}$ & $\mathrm{x}$ \\
\hline
\end{tabular}

${ }^{2}$ Values are from tabulated data for each $\theta$ given in the reference. Maximum test duration was 16 hrs.

${ }^{b}$ Values are generated from the regression equation given in the reference, which is valid between $750^{\circ} \mathrm{F}$ and $1300^{\circ} \mathrm{F}$, and for stress level below $15 \mathrm{ksi}$. Maximum duration of tests was not reported.

${ }^{c}$ Stress levels in the tests were between these two limits. Each test was conducted at constant load and temperature.

${ }^{d}$ Normalized by room temperature ultimate stress, values taken from [12]

${ }^{e}$ Assumes $\sigma_{u}(\theta)$ and $\sigma_{f}(\theta)$ have same ratios to their respective room temperature (RT) values, and $\sigma_{f}=100 \mathrm{ksi}$ at RT.

${ }^{f}$ From Fields and Fields (1989), who calculated $E, K, M$ on the basis that after a 2 min exposure, $\epsilon=\epsilon_{e}+\epsilon_{p}$ only. $\mathrm{x}=$ out of range

Table 4.3: Creep strain growth law parameters for ASTM A36 steel 


\begin{tabular}{|c|cccc|ccccc|}
\hline $\begin{array}{c}\theta \\
\left({ }^{\circ} \mathrm{F}\right)\end{array}$ & $\begin{array}{c}A \\
(\mathrm{MPa}, \mathrm{hr})\end{array}$ & $m$ & $\phi$ & $\begin{array}{c}\text { test } \sigma \\
(\mathrm{MPa})\end{array}$ & $\begin{array}{c}\sigma_{y}[26] \\
(\mathrm{MPa})\end{array}$ & $\begin{array}{c}\sigma_{f}[26] \\
(\mathrm{MPa})\end{array}$ & $\begin{array}{c}E[26] \\
(\mathrm{GPa})\end{array}$ & $\begin{array}{c}K \\
(\mathrm{MPa})\end{array}$ & $M$ \\
\hline$R T$ & - & - & - & - & 275 & 595 & 193 & 891.4 & 4.22 \\
1100 & $2.32 \times 10^{-20}$ & 6.92 & 1 & $199-315$ & 152 & 443.7 & 151.6 & 492.7 & 4.22 \\
\hline
\end{tabular}

$A, m$ at $1100^{\circ} \mathrm{F}$ obtained from least square analysis of 36 test data from [43].

Room-temperature ( $R T$ ) $K, M$ obtained from least-squares fit on curve in [9]. Elevated temperature $K, M$ obtained with the assumption $K(\theta) / K(R T)=\sigma_{y}(\theta) / \sigma_{y}(R T)$ and $M(\theta)=M(R T)$, in the abserice of detailed information.

Table 4.4: Creep strain growth law parameters for type 316 stainless steel 


\begin{tabular}{|c|c|c|c|c|c|}
\hline \multirow{3}{*}{$\begin{array}{c}t_{f} \\
(\mathrm{hr})\end{array}$} & \multirow{3}{*}{$\begin{array}{c}\theta \\
\left({ }^{\circ} F\right)\end{array}$} & \multicolumn{4}{|c|}{ Failure stress (ksi) } \\
\hline & & \multicolumn{2}{|c|}{ Predicted } & \multicolumn{2}{|c|}{ Experimental } \\
\hline & & $\left(D_{c}=0.2\right)$ & $\left(D_{c}=1.0\right)$ & ref[13] & ref[12] \\
\hline \multirow[t]{3}{*}{1000} & 800 & 35.4 & 41.2 & 38.0 & 25.8 \\
\hline & 900 & $16.6^{*}$ & $17.1^{*}$ & 18.5 & 19.0 \\
\hline & 1000 & $13.1^{*}$ & $13.5^{*}$ & 9.5 & 11.7 \\
\hline \multirow[t]{3}{*}{10000} & 800 & 34.7 & 39.1 & 24.8 & 21.0 \\
\hline & 900 & $14.1^{*}$ & $14.5^{*}$ & 12.4 & 13.8 \\
\hline & 1000 & $10.5^{*}$ & $10.9^{*}$ & 6.3 & 6.8 \\
\hline \multirow[t]{3}{*}{100000} & 800 & 33.5 & 36.8 & 16.0 & - \\
\hline & 900 & $12.0^{*}$ & $12.3^{*}$ & 8.2 & - \\
\hline & 1000 & $8.5^{*}$ & $8.7^{*}$ & 4.2 & - \\
\hline
\end{tabular}

* using out of range parameters

- value not reported

Table 4.5: Experimental and predicted failure stresses due to creep in ASTM A36 steel using Fields and Fields data 


\begin{tabular}{|c|c|c|c|c|c|}
\hline \multirow{3}{*}{$\begin{array}{c}t_{f} \\
(\mathrm{hr})\end{array}$} & \multirow{3}{*}{$\begin{array}{c}\theta \\
\left({ }^{\circ} F\right)\end{array}$} & \multicolumn{4}{|c|}{ Failure stress (ksi) } \\
\hline & & \multicolumn{2}{|c|}{ Predicted } & \multicolumn{2}{|c|}{ Experimental } \\
\hline & & $\left(D_{c}=0.2\right)$ & $\left(D_{c}=1.0\right)$ & $\operatorname{ref}[13]$ & ref[12] \\
\hline \multirow[t]{3}{*}{1000} & 800 & $35.1^{*}$ & $37.4^{*}$ & 38.0 & 25.8 \\
\hline & 900 & $21.5^{*}$ & $22.7^{*}$ & 18.5 & 19.0 \\
\hline & 1000 & 11.3 & 12.0 & 9.5 & 11.7 \\
\hline \multirow[t]{3}{*}{10000} & 800 & $28.6^{*}$ & $30.4^{*}$ & 24.8 & 21.0 \\
\hline & 900 & 14.5 & $15.4^{*}$ & 12.4 & 13.8 \\
\hline & 1000 & 7.6 & 8.0 & 6.3 & 6.8 \\
\hline \multirow[t]{3}{*}{100000} & 800 & $20.6^{*}$ & $21.7^{*}$ & 16.0 & - \\
\hline & 900 & 9.7 & 10.3 & 8.2 & - \\
\hline & 1000 & 5.1 & 5.4 & 4.2 & - \\
\hline
\end{tabular}

* using out of range parameters

- value not reported

Table 4.6: Experimental and predicted failure stresses due to creep in ASTM A36 steel using Harmathy data 


\begin{tabular}{|c|c|c|c|c|c|c|c|c|c|c|c|c|}
\hline \multirow{3}{*}{$\begin{array}{c}\theta \\
\left(^{\circ} F\right)\end{array}$} & \multirow{3}{*}{$\begin{array}{c}\sigma_{\infty} \\
(\mathrm{MPa})\end{array}$} & \multirow{2}{*}{\multicolumn{2}{|c|}{$\begin{array}{l}\text { Predicted } t_{f} \\
D_{c}(\text { eq } 4.56)\end{array}$}} & \multicolumn{9}{|c|}{ Experimental (ref [43]), hours } \\
\hline & & & & \multicolumn{3}{|c|}{$t_{1}$ (primary) } & \multicolumn{3}{|c|}{$t_{2}($ secondary $)$} & \multicolumn{3}{|c|}{$t_{R}$ (rupture) } \\
\hline & & 0.2 & 1.0 & $\min$ & mean & $\max$ & $\min$ & mean & $\max$ & $\min$ & mean & $\max$ \\
\hline \multirow[t]{6}{*}{1100} & 199 & 841 & 1032 & 140 & 229 & 305 & 960 & 1283 & 1950 & 1267 & 1749 & 2437 \\
\hline & 218 & 384 & 477 & 20 & 46 & 88 & 150 & 325 & 546 & 170 & 439 & 779 \\
\hline & 239 & 167 & 211 & 8 & 12 & 17 & 60 & 77 & 99 & 76 & 105 & 132 \\
\hline & 262 & 69 & 89 & 3 & 4.3 & 6 & 17 & 27 & 31 & 22 & 37 & 43 \\
\hline & 288 & 25 & 34 & 0.9 & 1.9 & 3.8 & 4 & 9.4 & 15 & 6.6 & 13.3 & 20.4 \\
\hline & 315 & 8 & 12 & 0.3 & 0.5 & 0.7 & 1.3 & 3.3 & 6.2 & 1.9 & 4.9 & 9.0 \\
\hline
\end{tabular}

The experimental min,mean,max values pertain to 6 data points ( 2 each from three creep-rupture machines) for each stress level.

Table 4.7: Experimental and predicted failure times (hours) due to creep in type 316 stainless steel. 


\begin{tabular}{|c|c|c|c|c|c|c|}
\hline Material & source & $\begin{array}{c}E \\
(\mathrm{GPa}) \\
\end{array}$ & $\begin{array}{c}H \\
(\mathrm{MPa}) \\
\end{array}$ & $M^{\prime}$ & $\begin{array}{c}\sigma_{f} \\
(\mathrm{MPa})\end{array}$ & $\begin{array}{c}S_{e} \\
(\mathrm{MPa}) \\
\end{array}$ \\
\hline $\begin{array}{c}\text { AISI } 316 \\
\text { stainless steel }\end{array}$ & $\begin{array}{c}\text { Boller \& } \\
\text { Seeger, } 1987\end{array}$ & 205 & 691 & 6.5 & $721^{a}$ & $155^{b}$ \\
\hline $\begin{array}{l}\text { AISI } 1015 \\
\text { steel }\end{array}$ & Dowling, 1993 & 206 & 1058 & 4.2 & 725 & $220^{c}$ \\
\hline $\begin{array}{c}\mathrm{A} 106 \mathrm{Gr}-\mathrm{B} \text { steel } \\
\left(288^{\circ} \mathrm{C} \text { in air }\right)\end{array}$ & $\begin{array}{c}\text { Chopra et al, } \\
1995^{f}\end{array}$ & 196.5 & 1994 & 7.74 & $539^{d}$ & $310^{e}$ \\
\hline $\begin{array}{l}\text { AISI } 4340 \\
\text { steel }\end{array}$ & $\begin{array}{c}\text { Endo \& Morrow } \\
1969\end{array}$ & 192.9 & 1812 & 7.1 & 1911 & $542^{g}$ \\
\hline
\end{tabular}

abased on $S_{u}=515 \mathrm{MPa}$ and $e_{f}=0.4$ from ASM Specialty Handbook: Stainless Steels, 1994; and assuming $\sigma_{f} \simeq S_{u}\left(1+e_{u}\right)$

${ }^{b}$ assuming $S_{e}=0.3 S_{u}$, suggested by Dowling, 1993

${ }^{c}$ based on $S_{e} \simeq 220 \mathrm{MPa}$ for 1020 steel from Collins, 1981

${ }^{d}$ based on $S u=415 \mathrm{MPa}$ at room temp ([46]vol 1, p. 332) and $S_{u}\left(288^{\circ} C\right)=1.1 S_{u}(R T)($ ref $[12])$ and $\sigma_{f} \simeq S_{u}\left(1+e_{u}\right)$

efrom Majumdar et al, 1993

${ }^{f}$ Environmentally Assisted Cracking of Light Water Reactors, NUREG/CR-4667.

${ }^{g}$ from Topper and Morrow, 1970

Table 4.8: Cyclic material properties of various alloys 


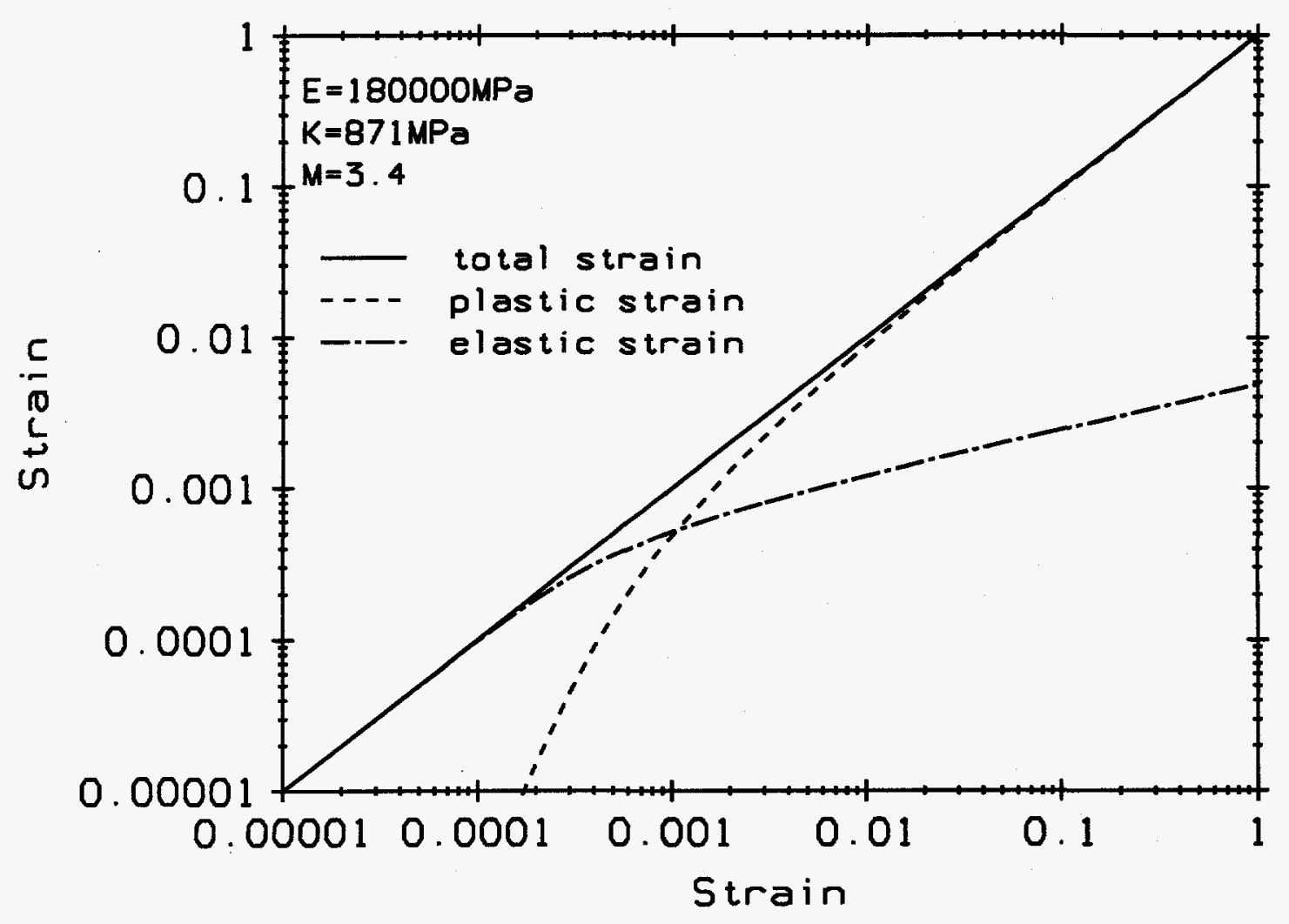

Figure 4.1: Elastic and plastic components of total strain 

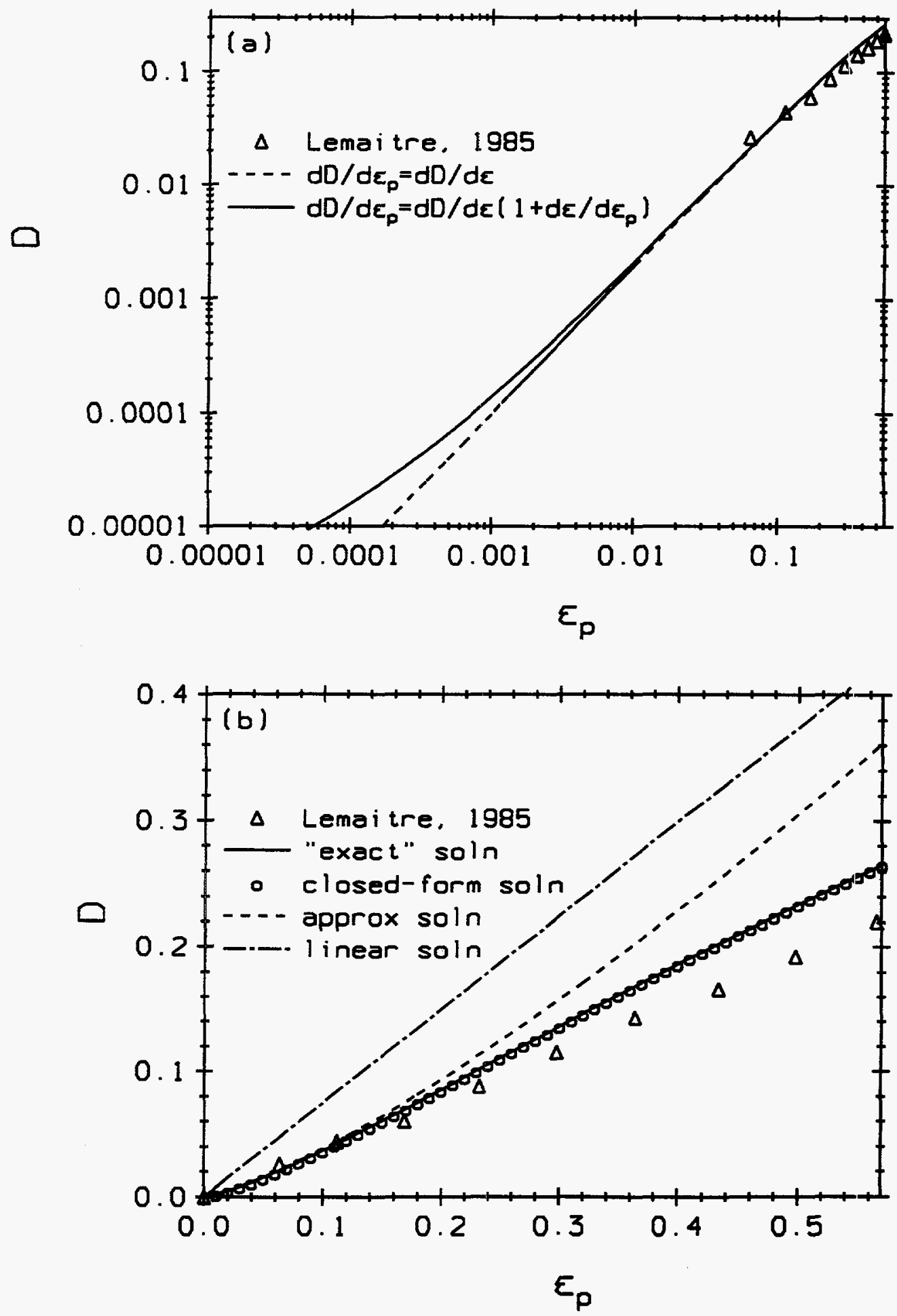

Figure 4.2: Ductile damage growth in SAE 1035 steel

NUREG/CR-6546

80 


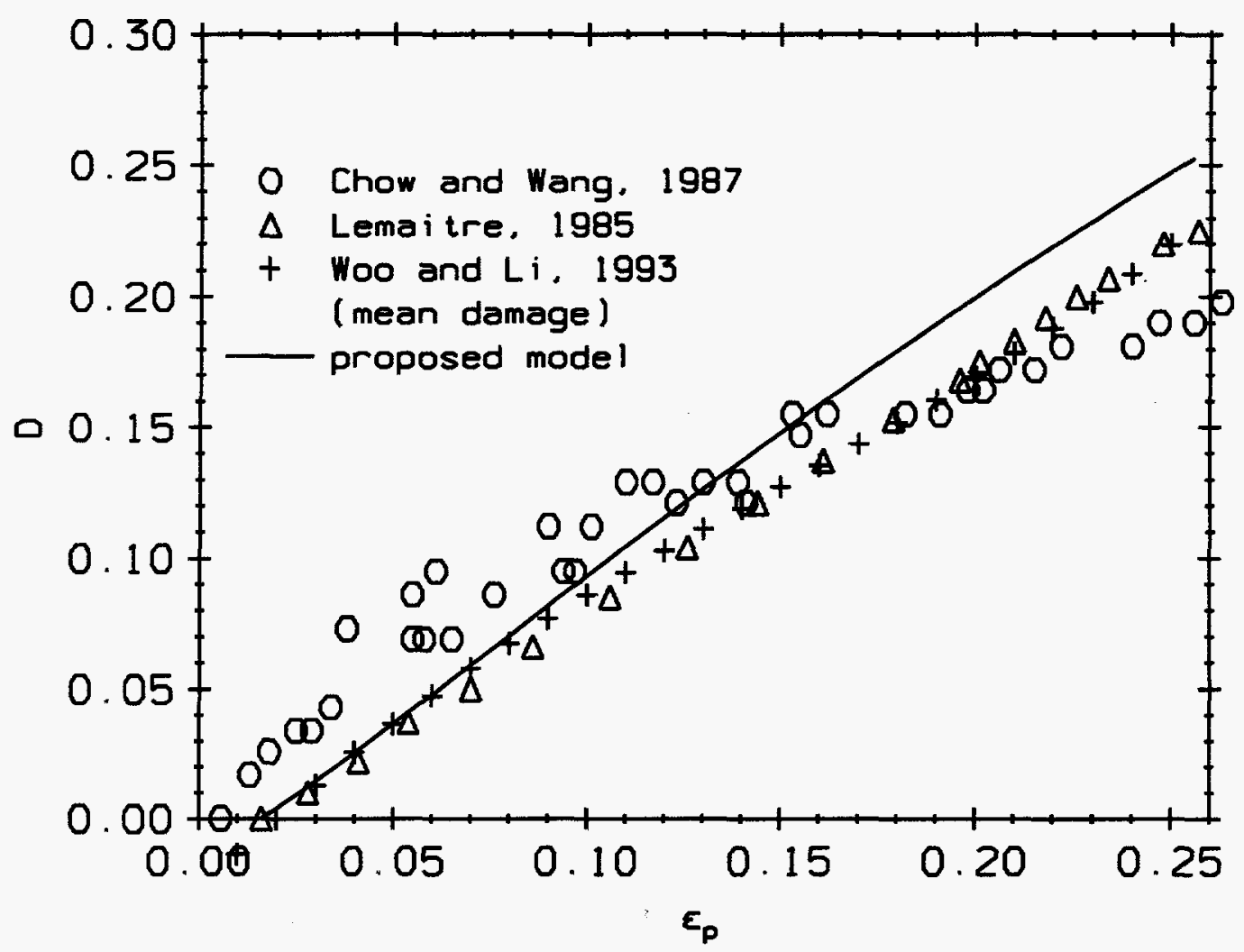

Figure 4.3: Ductile damage growth in 2024-T3 aluminum 


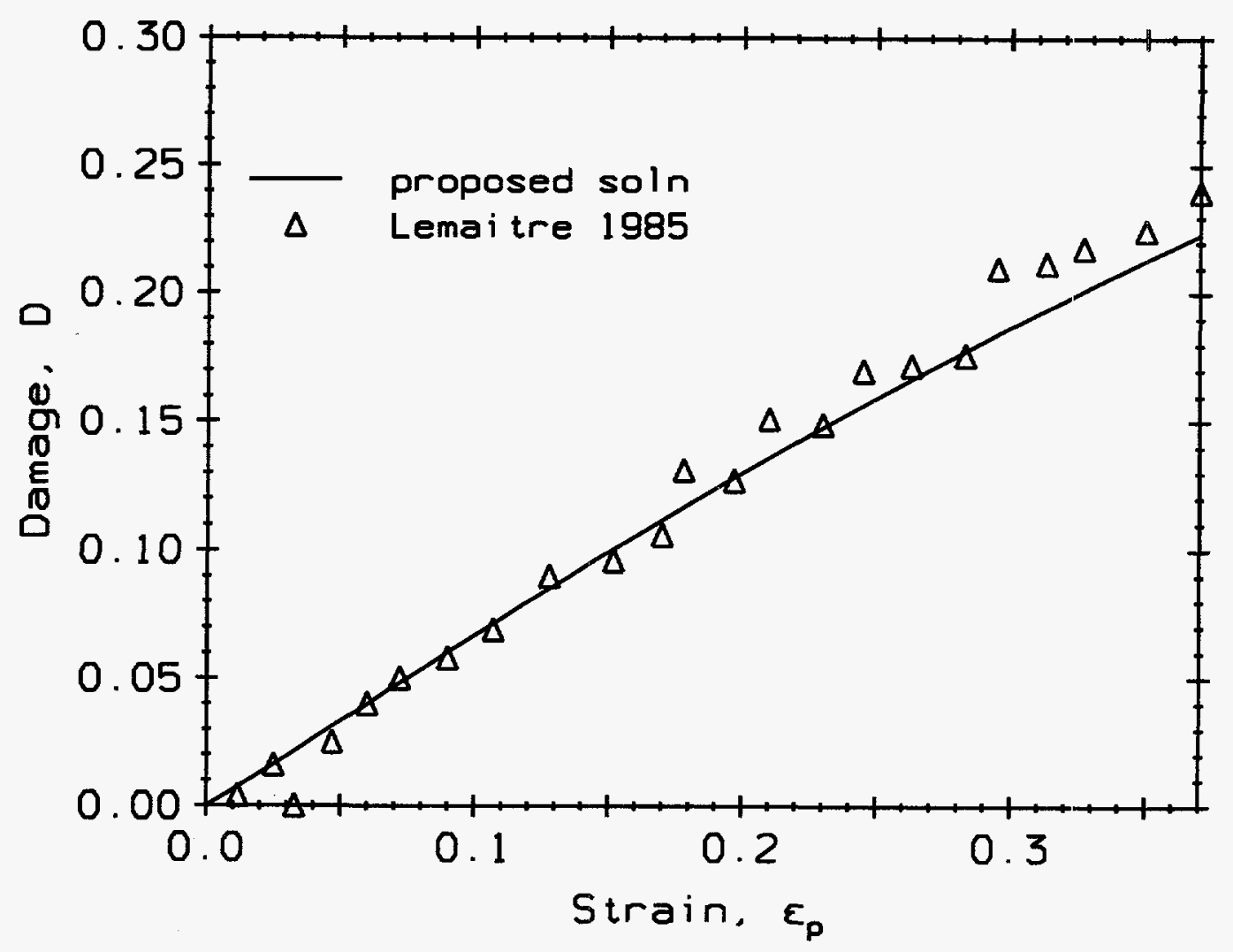

Figure 4.4: Ductile damage growth in AISI 4130 steel 


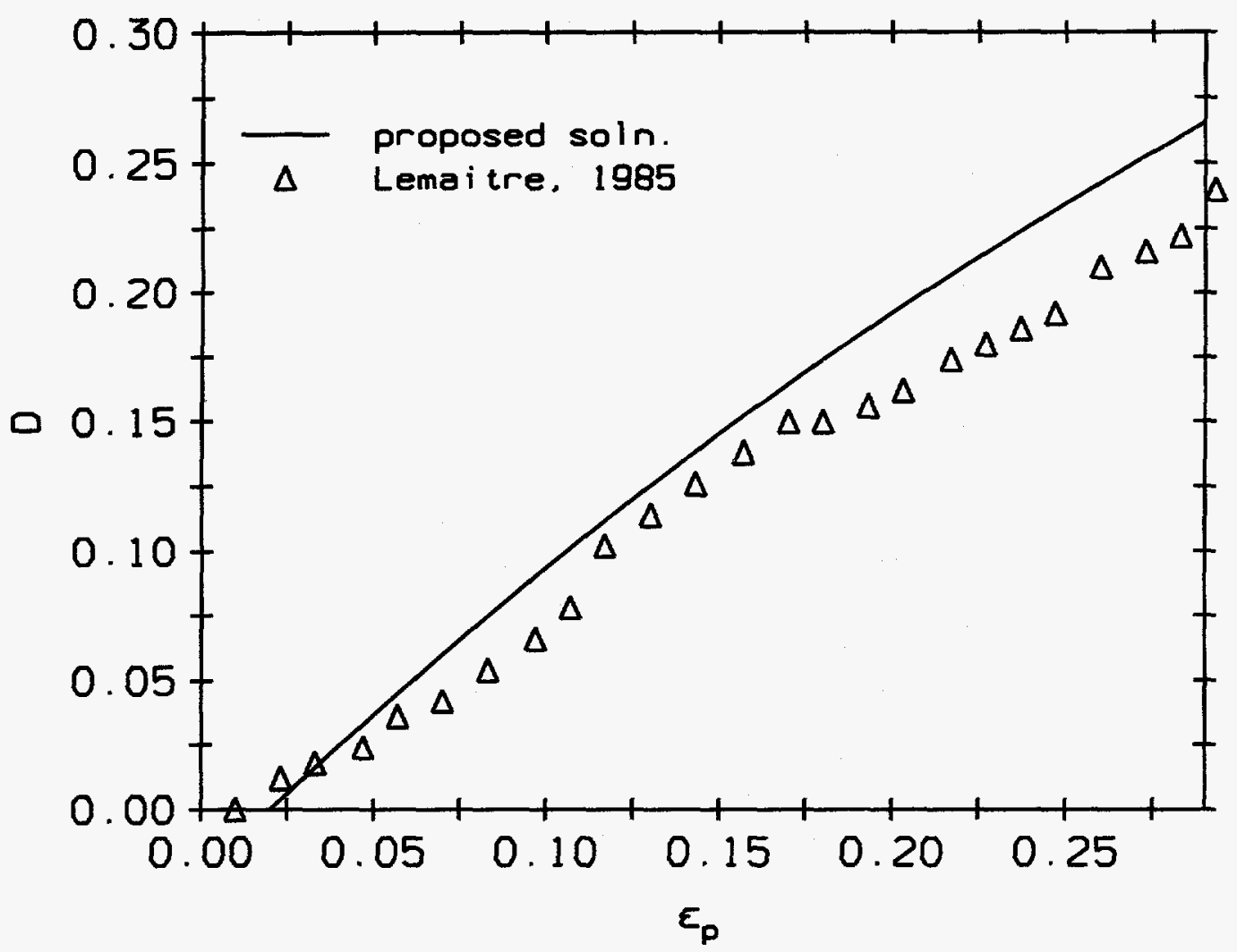

Figure 4.5: Ductile damage growth in INCO 718 alloy 


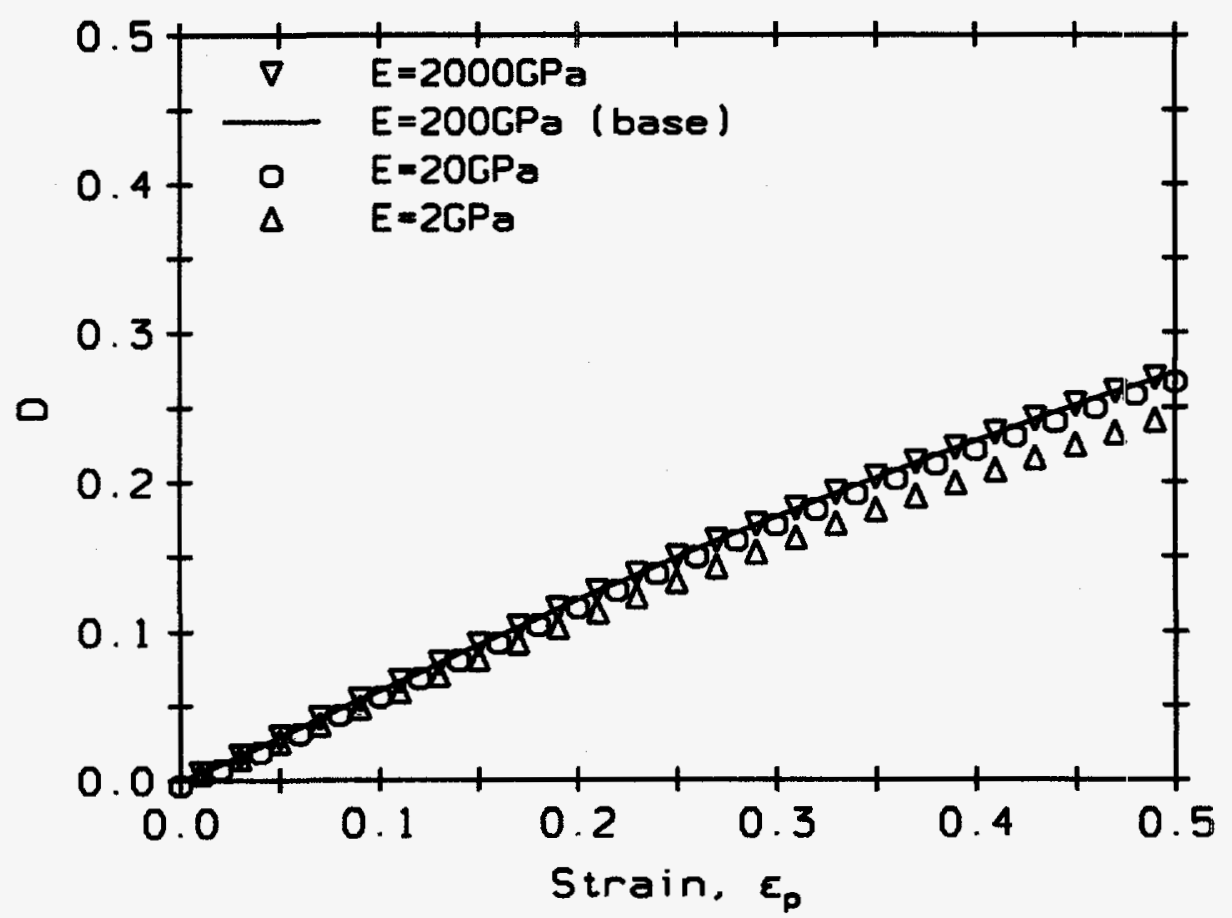

Figure 4.6: Sensitivity of proposed ductile damage growth model to $E$

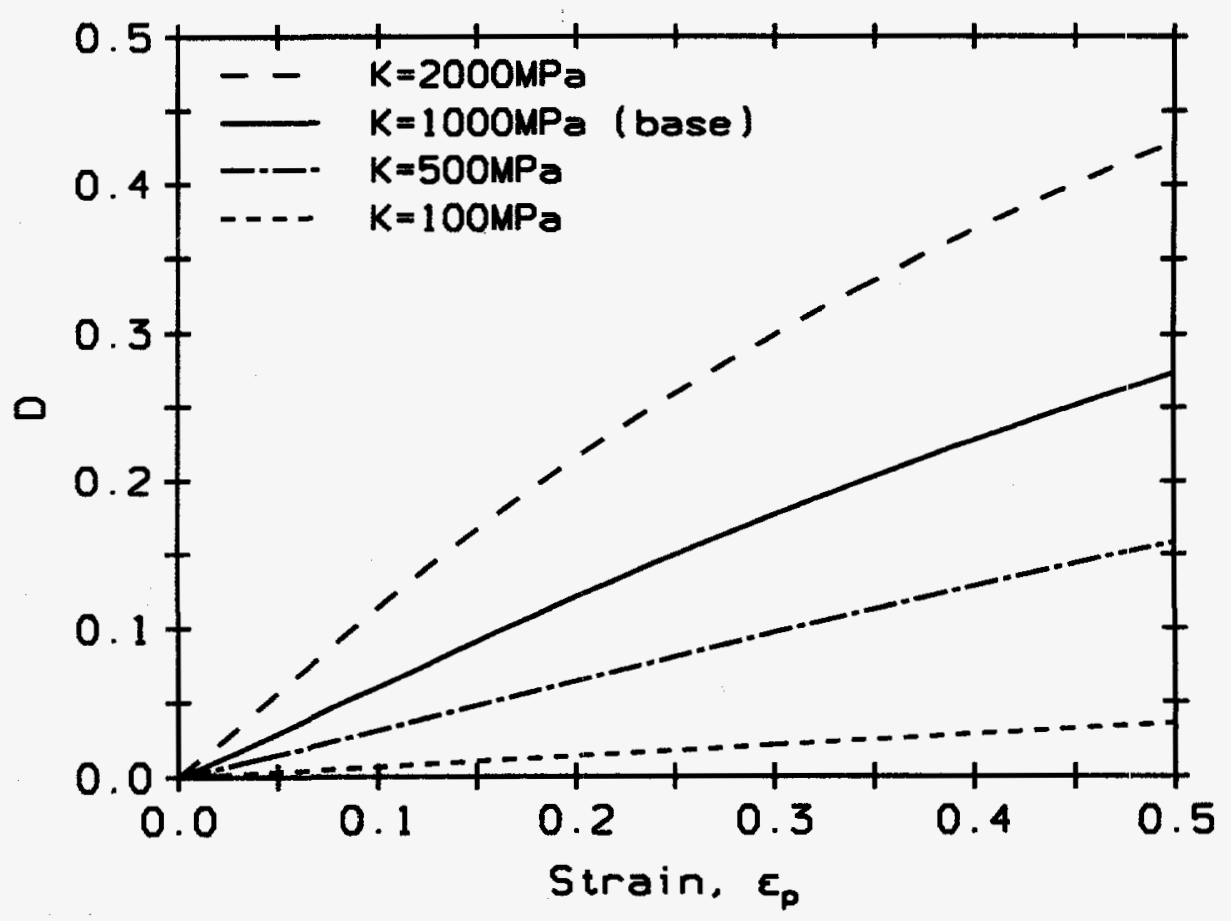

Figure 4.7: Sensitivity of proposed ductile damage growth model to $K$ 


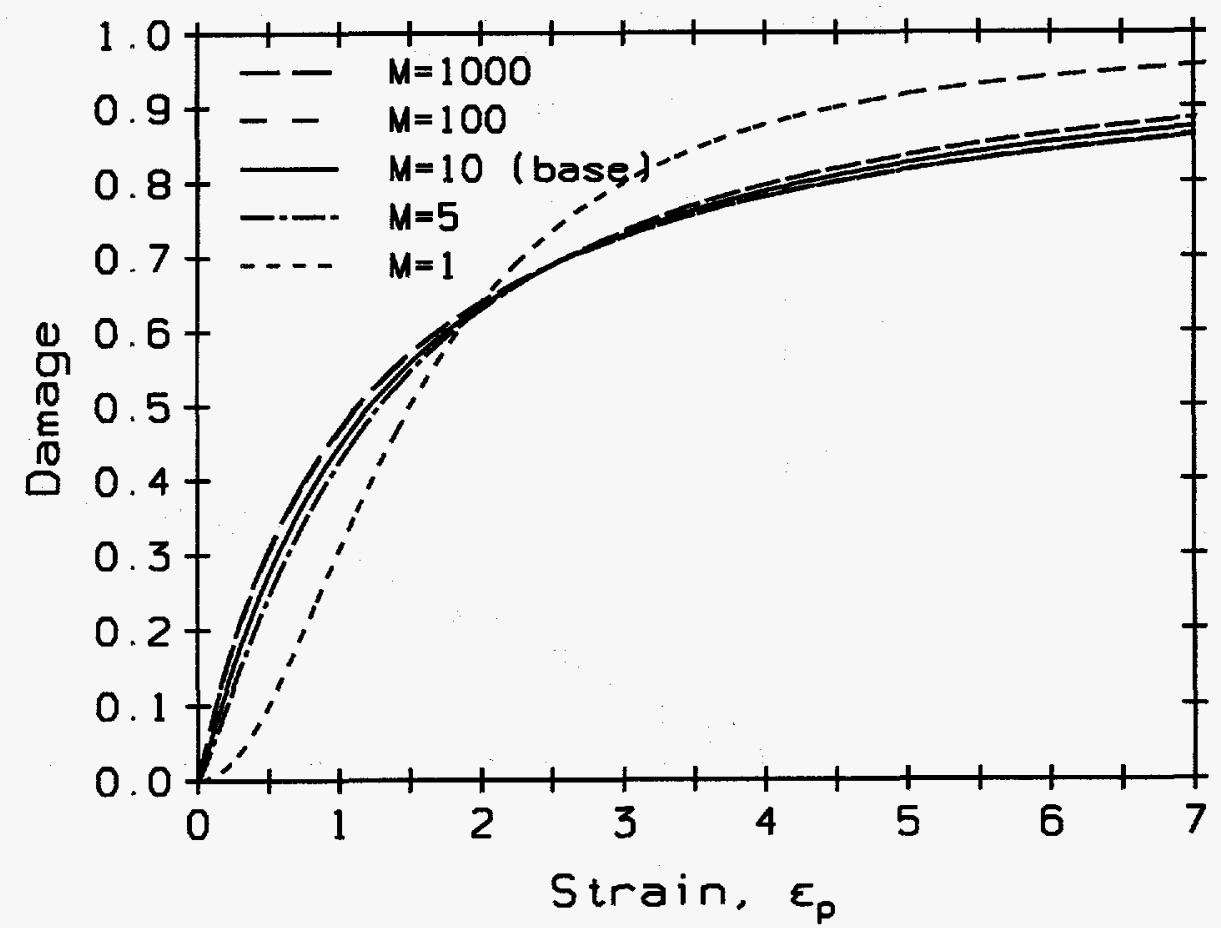

Figure 4.8: Sensitivity of proposed ductile damage growth model to $M$

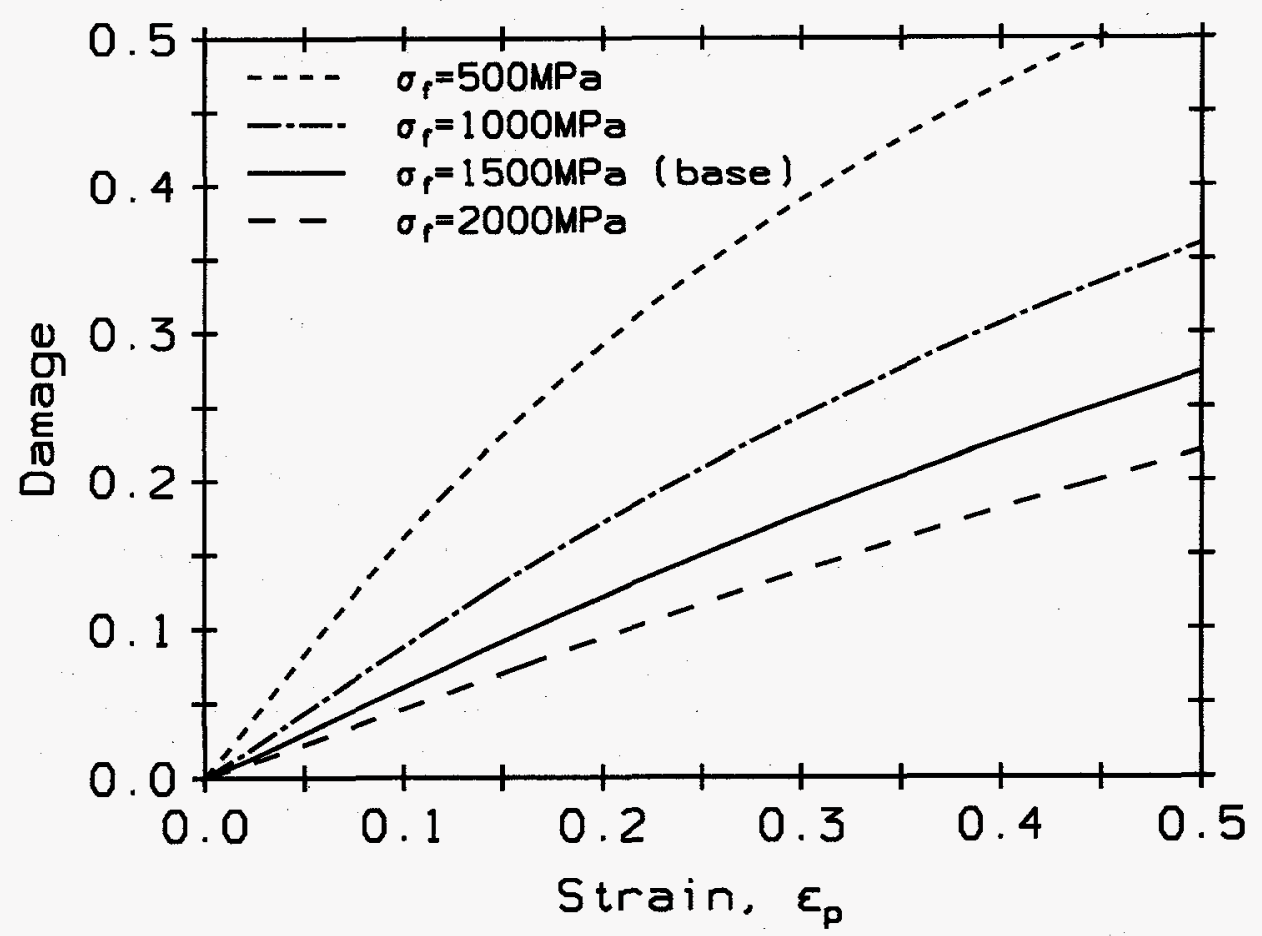

Figure 4.9: Sensitivity of proposed ductile damage growth model to $\sigma_{f}$ 


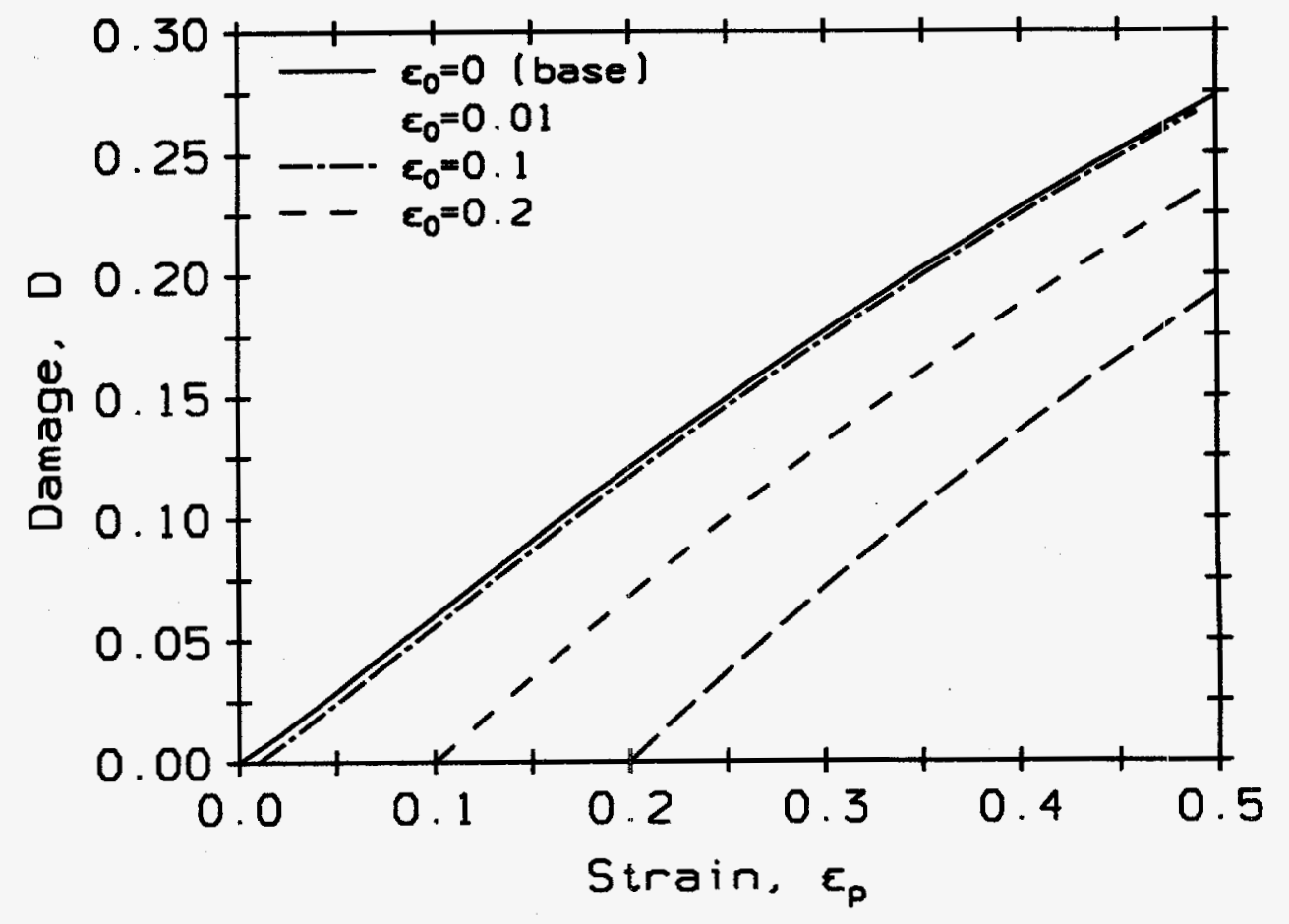

Figure 4.10: Sensitivity of proposed ductile damage growth model to $\epsilon_{0}$ 


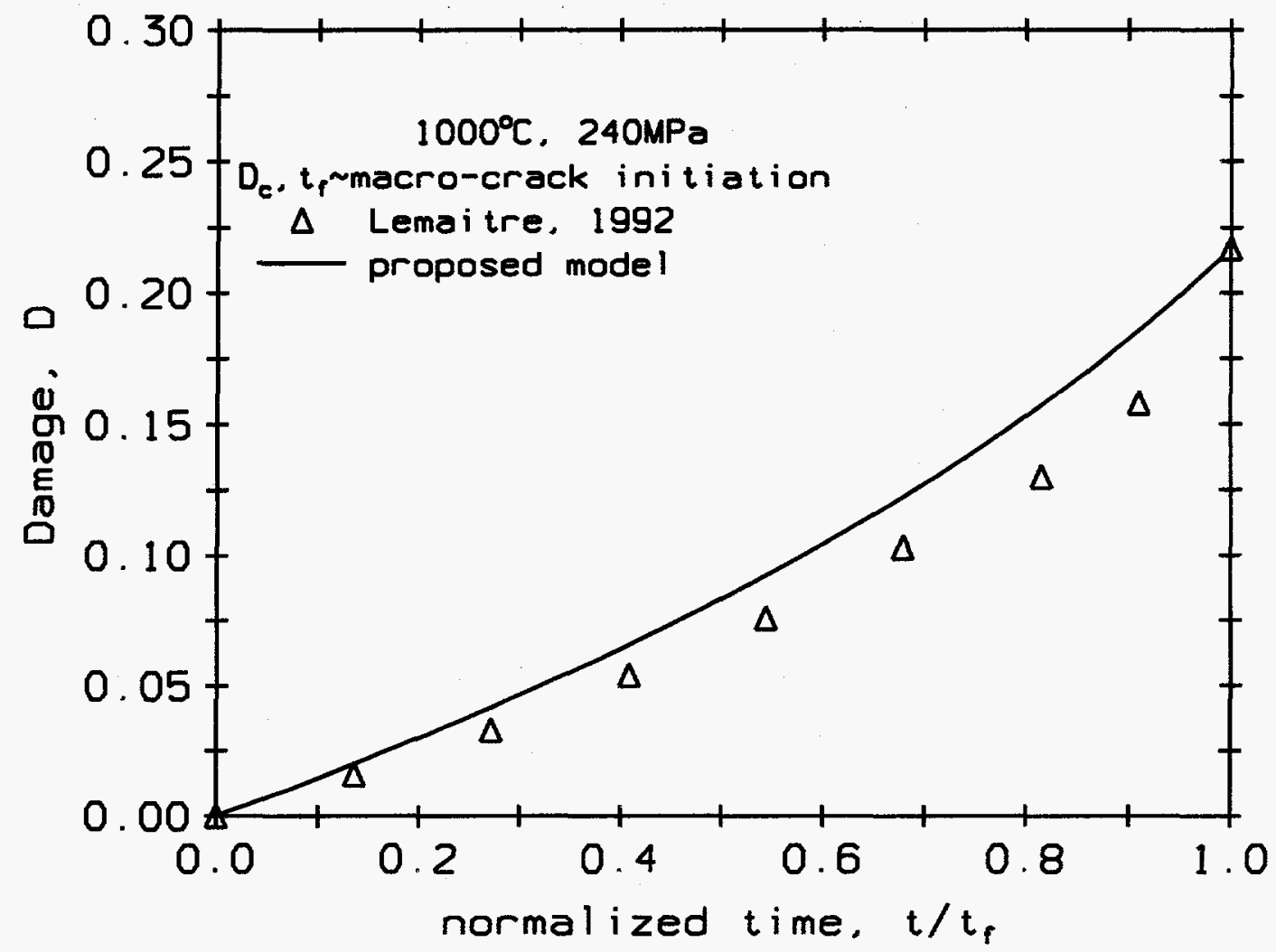

Figure 4.11: Creep damage growth in IN 100 superalloy 


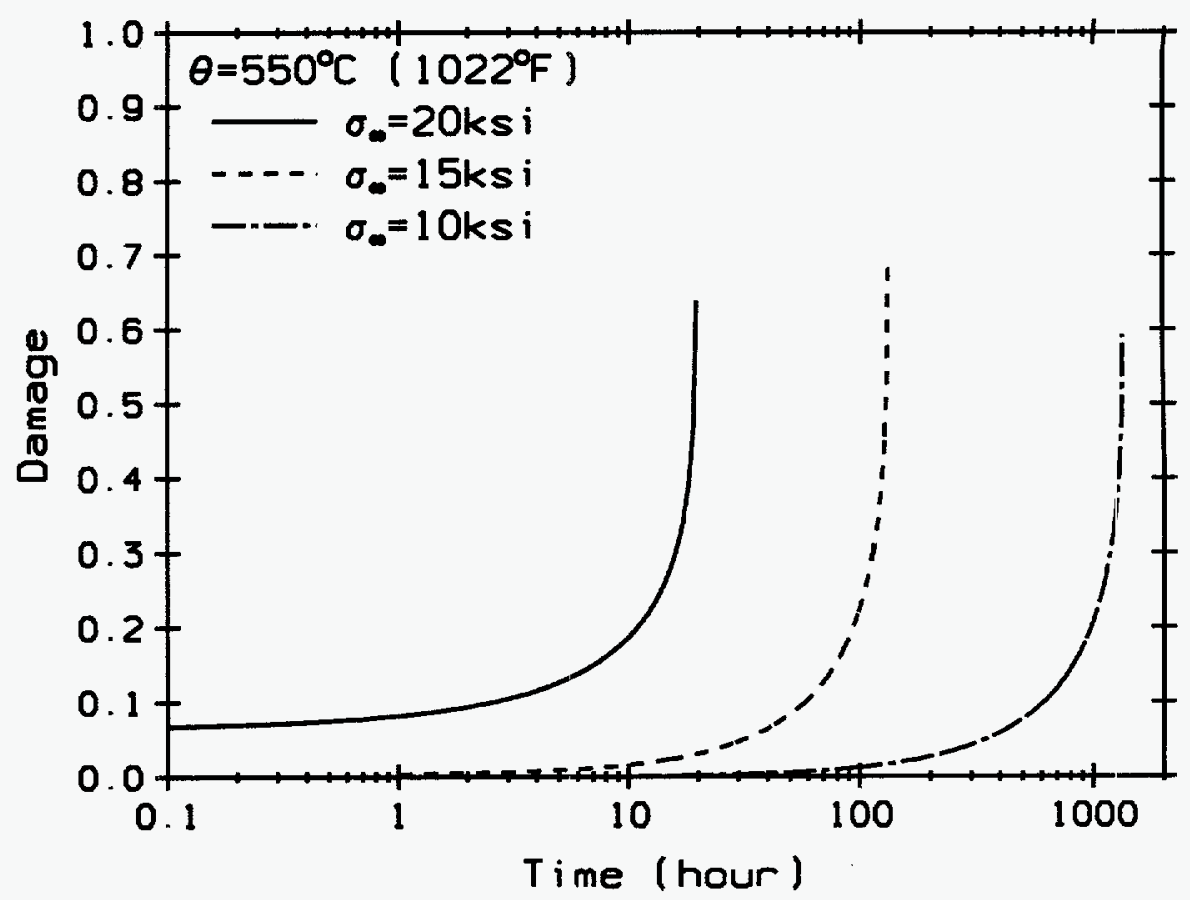

Figure 4.12: Effect of applied stress on predicted creep damage growth in ASTM A36 steel at constant temperature. (Model parameters taken from [41] when $\sigma=20 \mathrm{ksi}$ (138MPa), and from [49] when $\sigma=15 k s i(103.5 M P a)$ and $10 \mathrm{ksi}(69 \mathrm{MPa})$.)

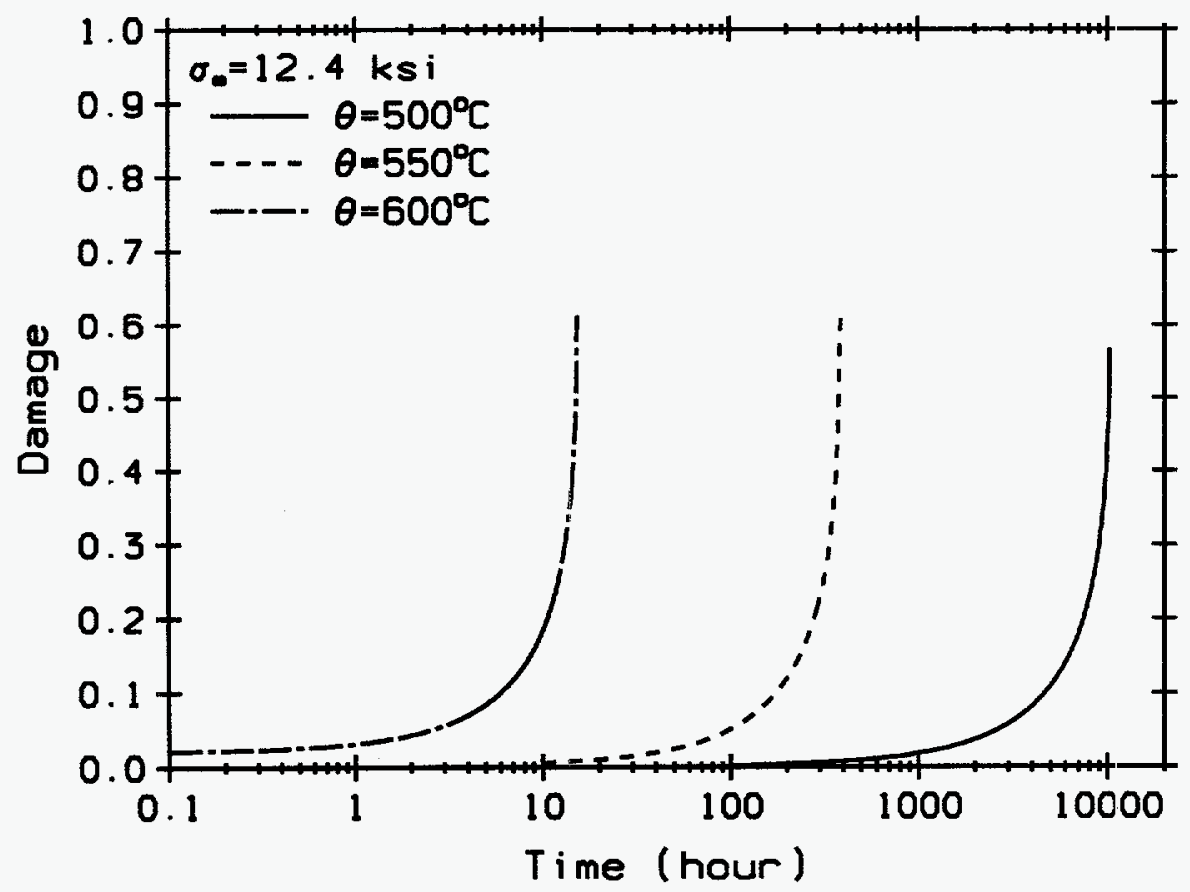

Figure 4.13: Effect of temperature on predicted creep damage growth in ASTM A36 steel at constant stress. (Model parameters taken from [49]). 


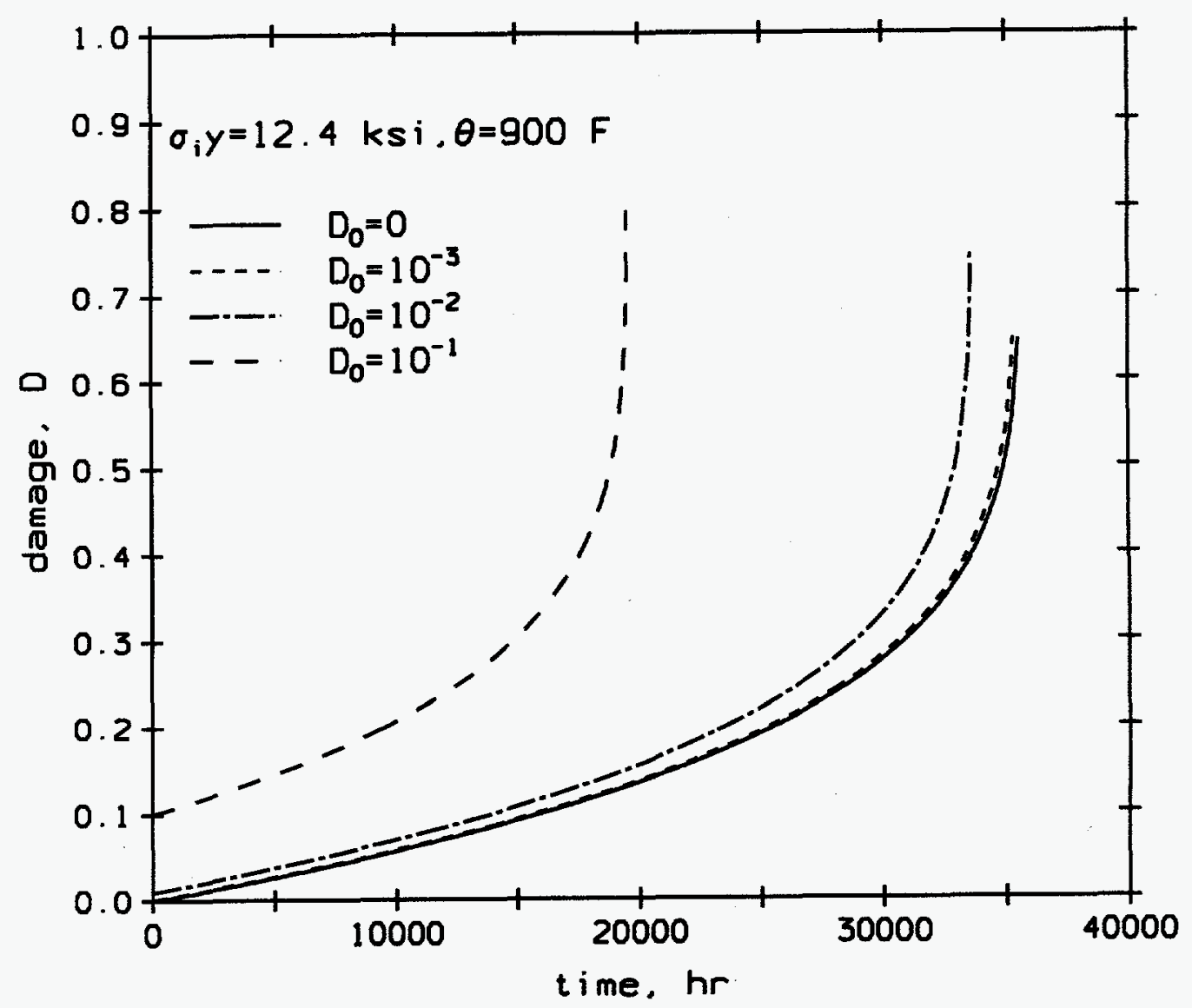

Figure 4.14: Effect of $D_{0}$ on predicted creep damage growth in ASTM A36 steel at constant stress. (Model parameters taken from [49]) 


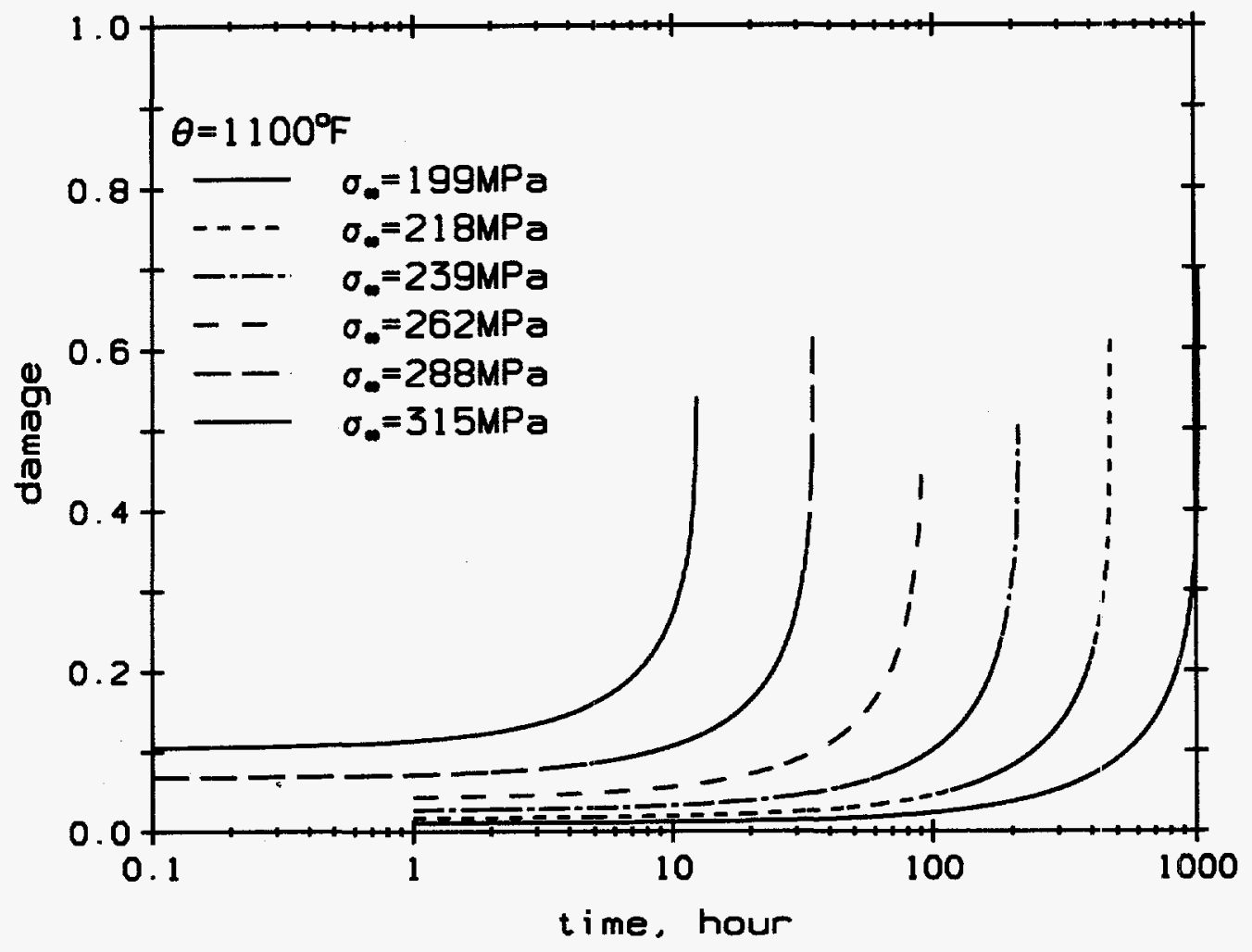

Figure 4.15: Predicted creep damage growth in type 316 stainless steel under different stress levels at constant temperature, data from [43]. 


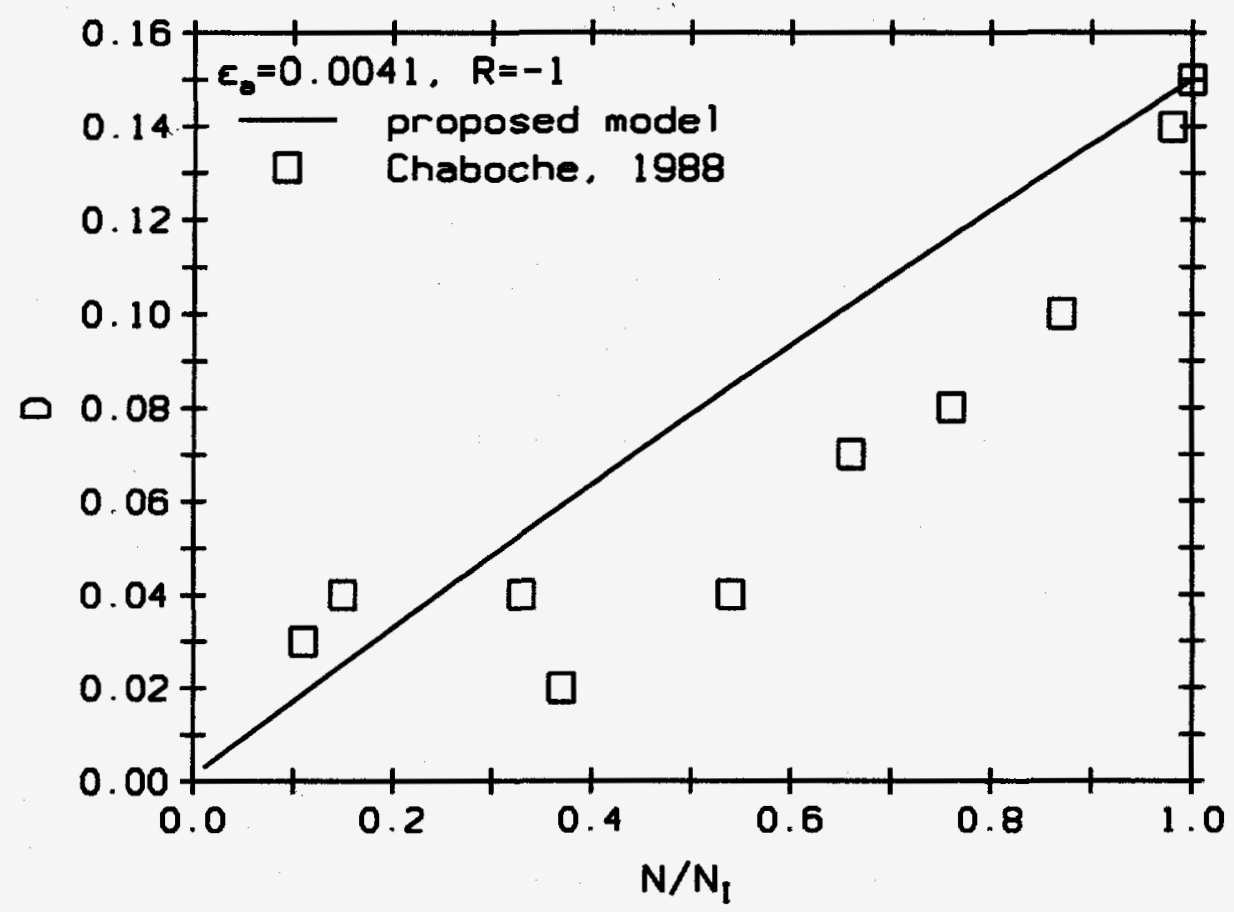

Figure 4.16: Predicted and experimental strain-controlled fatigue damage growth in type 316 stainless steel 


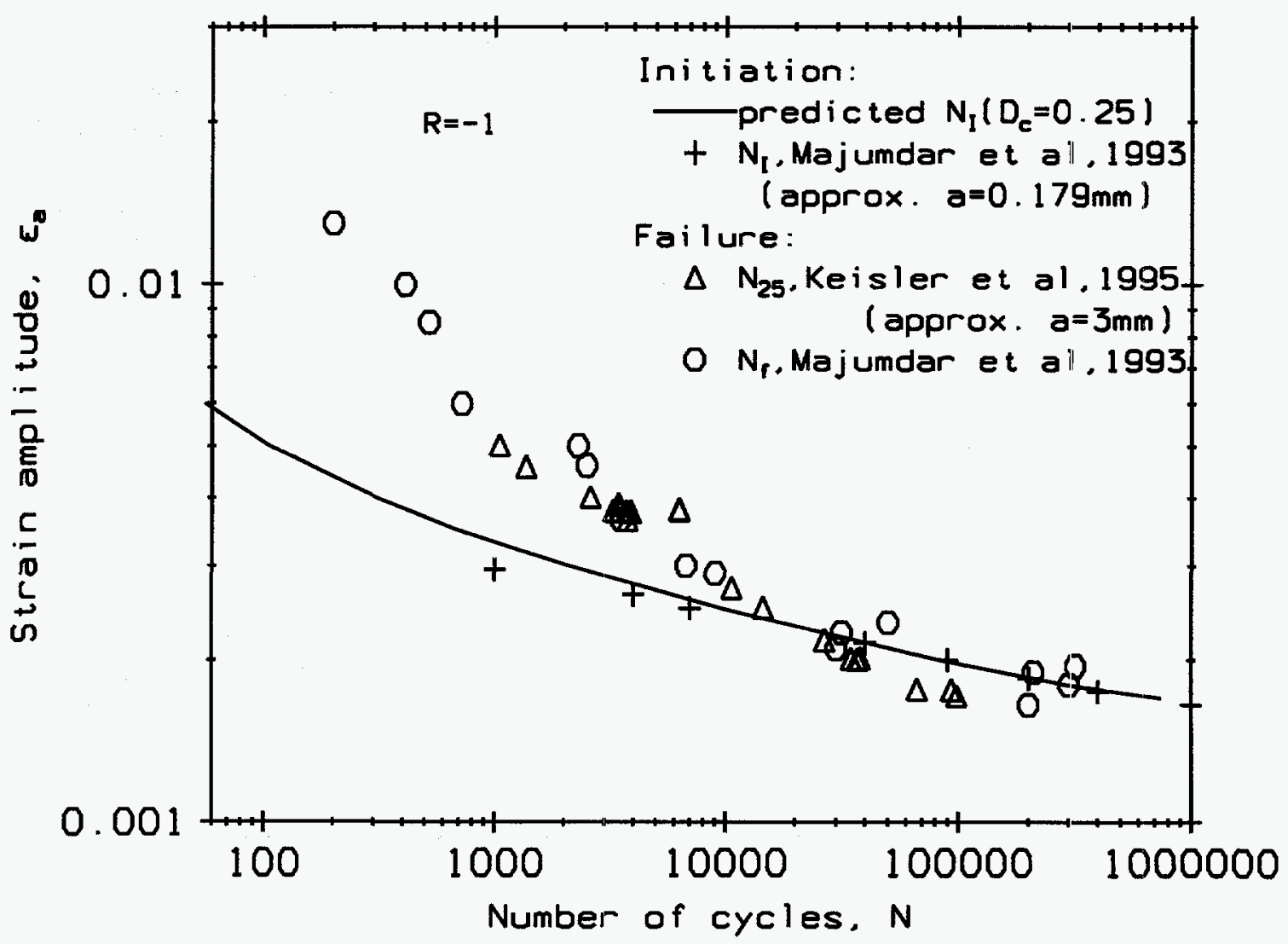

Figure 4.17: Predicted and experimental fatigue damage growth in A106-Gr B steel at $288^{\circ} \mathrm{C}$ in air 


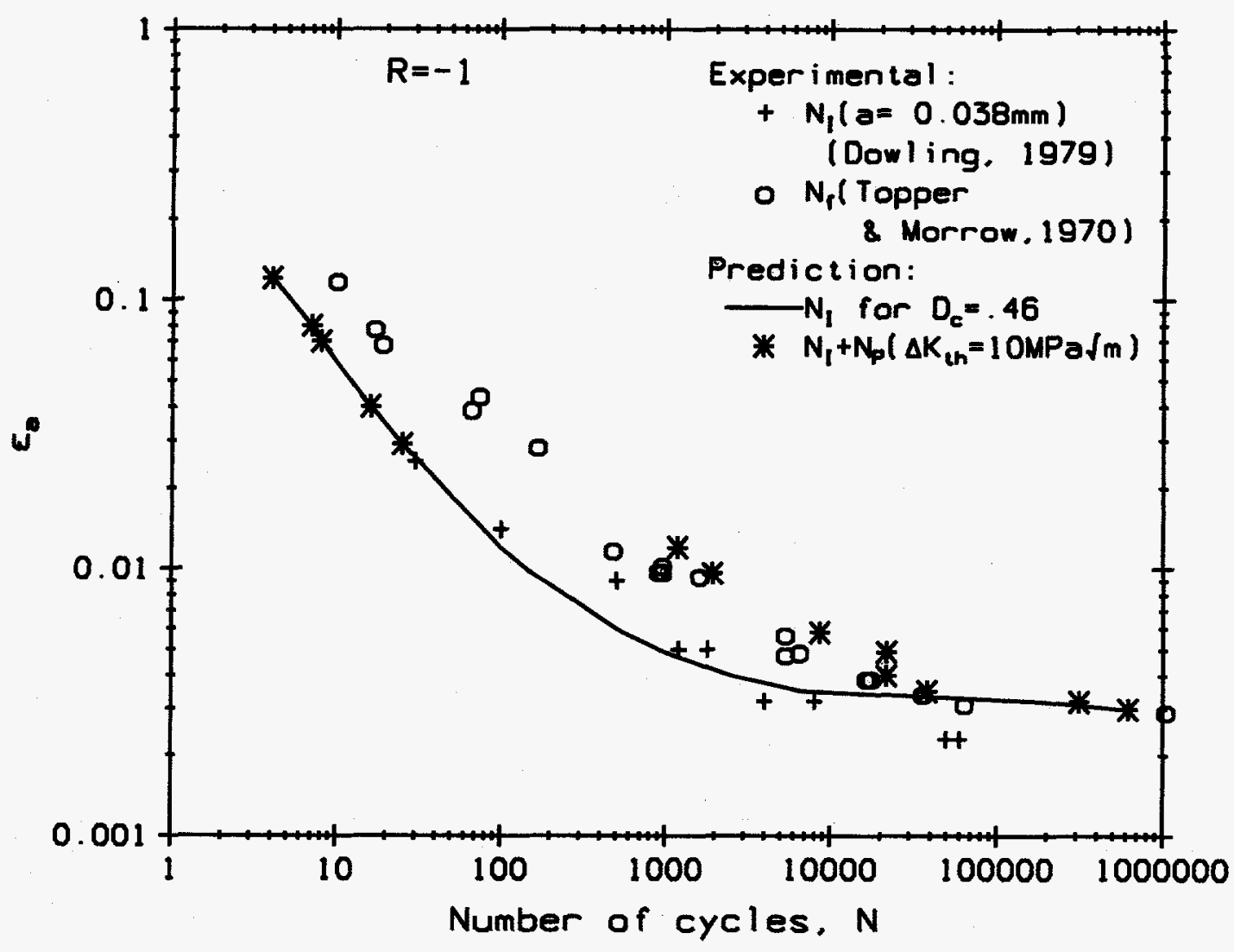

Figure 4.18: Predicted and experimental fatigue damage growth in SAE 4340 steel at room temperature in air 


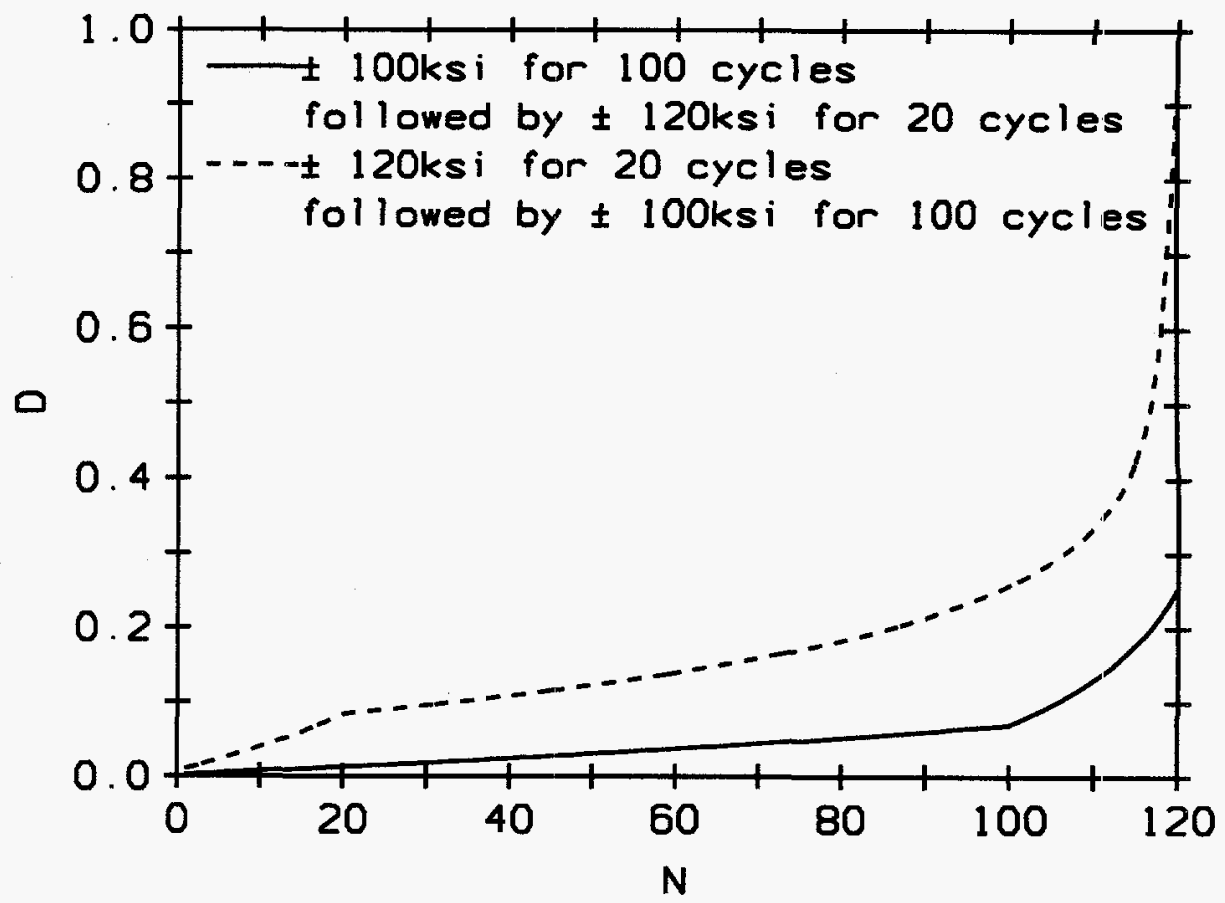

Figure 4.19: Effect of load sequencing on damage growth in stress-controlled fatigue of SAE 4340 steel

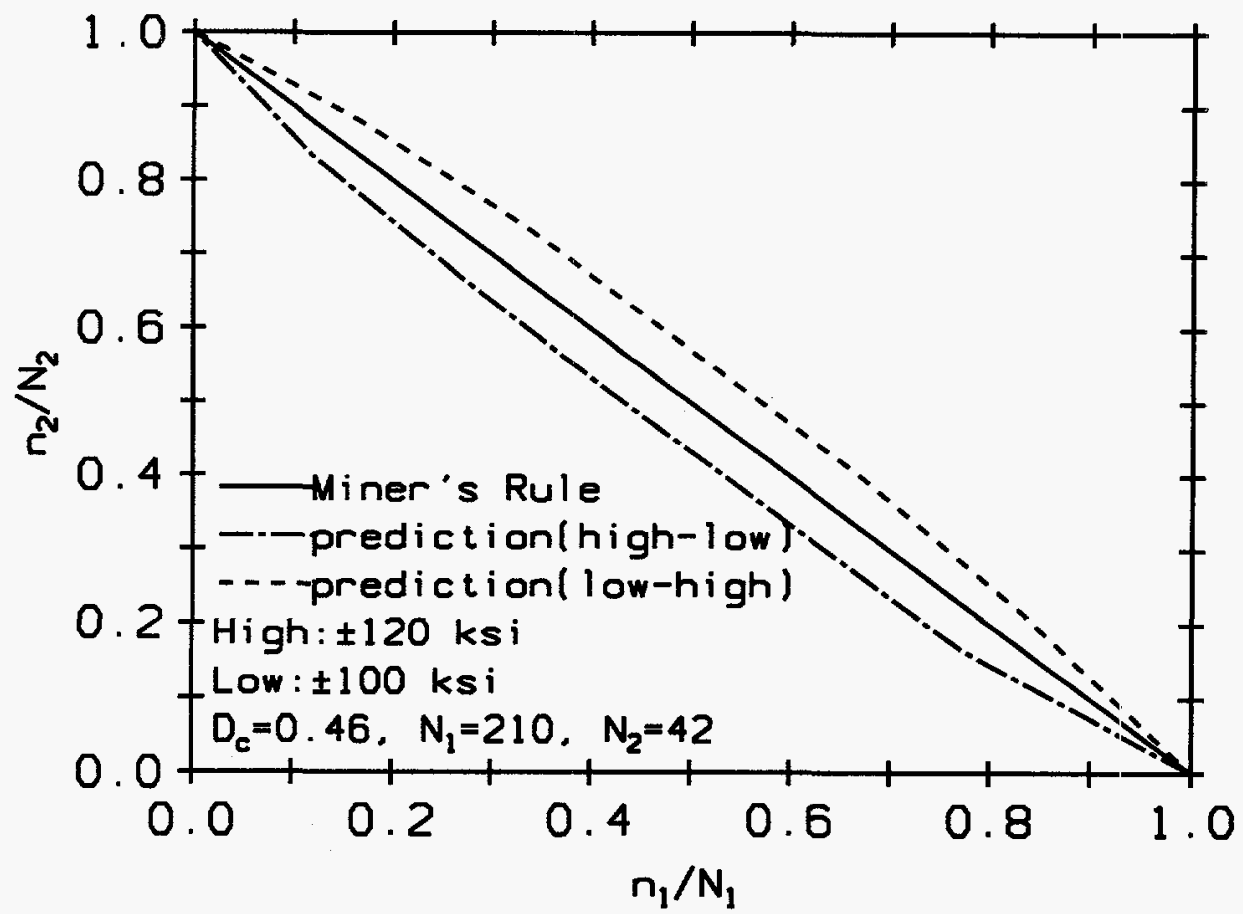

Figure 4.20: Predicted failure (i.e., crack initiation) in two-level stress-controlled fatigue cycling of SAE 4340 steel 


\section{Chapter 5}

\section{Stochastic CDM and Structural Reliability}

\subsection{Introduction}

In chapter 4, the growth laws governing isotropic damage due to inelastic deformation, high-temperature creep and fatigue were derived from the basic principles of thermodynamics and mechanics. The general form of the differential equations for these three different mechanisms of damage growth is

$$
\frac{d D}{d t}=f(D, t ; \omega)
$$

where $\omega$ is the set of material parameters. The independent parameter $t$ represents time, number of cycles or strain as appropriate. These models are deterministic, i.e., nonrandom: There is no uncertainty associated either with the value of damage or with the rate of damage growth at any given time. The tacit assumptions involved in deriving the above models were: (i) the Helmholtz free energy per unit volume did not have any spatial variability, (ii) the material parameters were precisely known (iii) no uncertainty existed either in loading, or in the environment, (iv) the interaction between defects could be ignored, ( $v$ ) the shape and orientation of defects had no random constituent as they were idealized as spheres, and (vi) the radii of the above spheres were deterministic.

However, the state of damage within a structure and its growth rate are actually both random, owing to the intrinsic randomness in the material microstructure and local thermal fluctuations, coupled with the randomness in the loading process and the environment. Experimental evidence of randomness in damage growth may be obtained from Woo and Li (1993b) (see Fig. 4.3) and Chow and Wei (1991). Since continuum damage mechanics (CDM) deals with the effect of microstructural variations within a structure, it is well-suited as a basis for modeling the randomness in damage growth when its source is not localized in some point within the structure (e.g., at the tip of 
the dominant crack). In other words, when the randomness in damage growth is the aggregate result of fluctuations arising throughout the material volume, a CDM-based approach is ideally suited to deal with it in a rational way.

In view of the randomness in damage growth, the deterministic models must be extended into the probabilistic domain to accommodate for the stochastic nature of damage accumulation. Equation (5.1) should therefore be generalized to:

$$
\frac{d D}{d t}=F(D, t ; \Omega)
$$

where is $D$ is now a stochastic process describing the random isotropic damage, $F$ is a random function representing damage growth rate, and $\Omega$ is the set of the random material parameters ${ }^{1}$.

The above randomization is done with the assumption that the sample functions of $D(t)$ are represented in some "average" manner by the non-random $\mathrm{D}(\mathrm{t})$ (the solution of eq 5.1); and that $F(\cdot)$ approaches the deterministic $f(\cdot)$ under some central tendency. Otherwise, the random damage growth law does not relate to its thermodynamic and mechanistic bases, and the randomization loses physical relevance. With this assumption in mind, we proceed to develop equations of stochastic damage growth. Certain stochastic concepts used in their development are introduced first.

\subsection{Fundamentals of Stochastic Differential Equations}

A stochastic process (or random process, or random function) $X(t)$ with $t \in\left[t_{0}, t_{f}\right]$ is a family of random variables that is indexed by the parameter ${ }^{2} t$. For a given value of $t$, the quantity $X_{t}=X(t)$ is a random variable with a probability distribution, $F_{X}(x ; t)$. The mean and the covariance are respectively the deterministic functions:

$$
\begin{aligned}
\mu_{X}(t) & =\mathrm{E}[X(t)] \\
K_{X X}\left(t_{1}, t_{2}\right) & =\operatorname{cov}\left[X\left(t_{1}\right), X\left(t_{2}\right)\right]=\mathrm{E}\left[X\left(t_{1}\right) X\left(t_{2}\right)\right]-\mu_{X}\left(t_{1}\right) \mu_{X}\left(t_{2}\right)
\end{aligned}
$$

When $t_{1}$ and $t_{2}$ coincide, $K_{X X}(t, t)$ reduces to the variance function $\sigma_{X}^{2}(t)$.

\section{Mean square continuity of a stochastic process}

If $X(t)$ is a stochastic process, the random variable $Y$ defined as

$$
Y(\delta)=X(t+\delta)-X(t)
$$

\footnotetext{
${ }^{1}$ In this chapter, uppercase letters will be used as much as practicable to denote the random versions of corresponding lowercase (deterministic) quantities.

${ }^{2}$ Since a large majority of stochastic processes represent temporally fluctuating quantities, the index is often referred to as "time" in a general discussion of stochastic processes where the context is unspecified.
} 
depends on the quantity $\delta$ and the instant of time, $t$. Its mean square value is

$$
\begin{aligned}
\mathrm{E}\left(Y^{2}\right) & =\mathrm{E}[(X(t+\delta)-X(t))(X(t+\delta)-X(t))] \\
& =\mathrm{E}\left[\{X(t+\delta)\}^{2}\right]-2 \mathrm{E}[X(t+\delta) X(t)]+\mathrm{E}\left[\{X(t)\}^{2}\right] \\
& =\sigma_{X}^{2}(t+\delta)+\mu_{X}^{2}(t+\delta)-2\left[K_{X X}(t+\delta, t)+\mu_{X}(t+\delta) \mu_{X}(t)\right]+\sigma_{X}^{2}(t)+\mu_{X}^{2}(t)
\end{aligned}
$$

If the covariance function of $X(t)$ is continuous at $(t, t)$, then the above expression vanishes as $\delta \rightarrow 0$ :

$$
\lim _{\delta \rightarrow 0} \mathrm{E}\left[\{Y(\delta)\}^{2}\right]=\lim _{\delta \rightarrow 0} \mathrm{E}\left[\{X(t+\delta)-X(t)\}^{2}\right]=0
$$

and the process $X(t)$ is said to be mean-square continuous at $t$. The necessary and sufficient condition for mean square continuity is that the covariance function $K_{X X}\left(t_{1}, t_{2}\right)$ is continuous at $(t, t)$.

\section{Integration of stochastic processes}

If $\phi(t)$ is a deterministic function and $X(t)$ is a stochastic process, the random variable, $Y$, given as

$$
Y=\int_{a}^{b} \phi(t) X(t) d t
$$

is called the integral of $\phi(t) X(t)$ if the infinite sum converges to $Y$ in some sense ${ }^{3}$. For example, mean-square integrability requires that (Lin, 1967):

$$
\left|\int_{a}^{b} \int_{a}^{b} \phi\left(t_{1}\right) \phi\left(t_{2}\right) K_{X X}\left(t_{1}, t_{2}\right) d t_{1} d t_{2}\right|<\infty
$$

Extending the above, if

$$
Z(\eta)=\int_{a}^{b} g(\eta, t) X(t) d t
$$

where $g$ is a deterministic function, then $Z(\eta)$ is a random process. The necessary and sufficient condition for the integral $Z(\eta)$ to exist in mean square is:

$$
\left|\int_{a}^{b} \int_{a}^{b} g\left(\eta_{1}, t_{1}\right) \overline{g\left(\eta_{2}, t_{2}\right)} K_{X X}\left(t_{1}, t_{2}\right) d t_{1} d t_{2}\right|<\infty
$$

in which $\bar{g}(\cdot)$ indicates the complex conjugate of the function $g(\cdot)$.

\footnotetext{
${ }^{3}$ Convergence of a sequence of random variables can occur in several ways, for example: with probability 1 , in probability, in distribution and in the mean square ( $\mathrm{Lin}, 1967)$; The latter is most commonly used in engineering applications.
} 


\section{Markov Processes}

$X(t)$ is a Markov process if, for $t_{n}>t_{n-1}>\ldots>t_{2}>t_{1} \geq 0$, its conditional probability density has the following property:

$$
\begin{gathered}
f_{X\left(t_{n}\right)}\left[x_{n} ; t_{n} \mid X\left(t_{n-1}\right)=x_{n-1} \cap X\left(t_{n-2}\right)=x_{n-2} \cap \ldots . . \cap X\left(t_{1}\right)=x_{1}\right] \\
=f_{X\left(t_{n}\right)}\left[x_{n} ; t_{n} \mid X\left(t_{n-1}\right)=x_{n-1}\right]
\end{gathered}
$$

and is called the transition probability density from time $t_{n-1}$ to $t_{n}$ of the Markov process. A process with independent increments in disjoint intervals is a Markov process. But this is a sufficient rather than a necessary condition for Markov processes since not all Markov processes have independent increments.

The future behaviour of a Markov process depends only on its current state. Knowledge about the past behaviour of a Markov process, that is, knowing the states occupied from the initiation up to the present, does not affect the probability distribution of the process at any time in the future if the present state is known. Physical processes which can be shown or reasoned to have this "memory-less" property may be treated as Markovian, allowing advantage to be taken of a rich collection of results pertaining to Markov processes.

\section{Diffusion processes}

A continuously parametered, continuous-state Markov process, $X(t)$, is termed a diffusion process if its transition probability density, $p(\cdot)$, meets the three conditions described below (Kloeden and Platen, 1992, p.36), in which $\epsilon>0, t_{2}>t_{1} \geq 0$ and $x_{1} \in \mathcal{R}$ :

1.

$$
\lim _{t_{2} \rightarrow t_{1}} \frac{1}{t_{2}-t_{1}} \int_{\left|x_{2}-x_{1}\right|>\epsilon} p\left(x_{2}, t_{2} \mid x_{1}, t_{1}\right) d x_{2}=0
$$

This condition prevents the process from having instantaneous jumps.

2. The instantaneous rate of change of the conditional expectation, $a\left(x_{1}, t_{1}\right)$, defined as

$$
\begin{aligned}
a\left(x_{1}, t_{1}\right) & =\lim _{t_{2} \rightarrow t_{1}} \frac{1}{t_{2}-t_{1}} \mathrm{E}\left[X\left(t_{2}\right)-X\left(t_{1}\right) \mid X\left(t_{1}\right)=x_{1}\right] \\
& =\lim _{t_{2} \rightarrow t_{1}^{+}} \frac{1}{t_{2}-t_{1}} \int_{\left|x_{2}-x_{1}\right|>\varepsilon}^{\left(x_{2}-x_{1}\right) p\left(x_{2}, t_{2} \mid x_{1}, t_{1}\right) d x_{2}}
\end{aligned}
$$

is a well-defined function. It is called the drift of the diffusion process at time $t_{1}$ and position $x_{1}$. 
3. The instantaneous rate of change of the squared fluctuations, $b\left(x_{1}, t_{1}\right)$, conditioned on $X\left(t_{1}\right)=x_{1}$ :

$$
\begin{aligned}
b\left(x_{1}, t_{1}\right) & =\lim _{t_{2} \rightarrow t_{1}^{+}} \frac{1}{t_{2}-t_{1}} \mathrm{E}\left[\left\{X\left(t_{2}\right)-X\left(t_{1}\right)\right\}^{2} \mid X\left(t_{1}\right)=x_{1}\right] \\
& =\lim _{t_{2} \rightarrow t_{1}} \frac{1}{t_{2}-t_{1}} \int_{\left|x_{2}-x_{1}\right|>\varepsilon}\left(x_{2}-x_{1}\right)^{2} p\left(x_{2}, t_{2} \mid x_{1}, t_{1}\right) d x_{2}
\end{aligned}
$$

is a well-defined function. It is called the diffusion coefficient of the process at time $t_{1}$ and position $x_{1}$.

Heuristically, $X(t)$ can be conceived of as a system moving with a deterministic velocity, $a(x, t)$, on which is superimposed a zero-mean Gaussian fluctuation, $\eta(t)$, with variance $b(x, t)$ :

$$
X(t+\Delta \dot{t})=X(t)+a(x, t) \Delta t+\eta(t) \sqrt{\Delta t}
$$

It is easily seen that the sample paths are continuous (as $\Delta t \rightarrow 0, x(t+\Delta t) \rightarrow x(t)$ ) but they are nowhere differentiable due to the presence of the term $\sqrt{\Delta t}$ (Gardiner 1985, p. $53)$.

The standard Wiener process, $W(t)$, is a diffusion process with zero drift and unit diffusion coefficient. It is a zero-mean Gaussian process, with variance $t$ and covariance function $K_{W W}\left(t_{1}, t_{2}\right)=\min \left(t_{1}, t_{2}\right) . W(t)$ is a non-stationary process with independent increments in disjoint intervals of time.

\section{Stochastic differential equations}

The integral of the white noise, $\xi(t)$, can be shown to be a Wiener process, $W(t)$ :

$$
d W(t)=W(t+d t)-W(t)=\xi(t) d t
$$

It should be noted that to derive the above, the white noise, $\xi(t)$, need not be Gaussian. The only requirement is that its autocovariance is a delta function (Gardiner, pp 81-83). $\mathrm{Eq}$ (5.17) leads to the idea of a "stochastic" differential equation (SDE) in the following way. An ordinary differential equation

$$
\frac{d x}{d t}=a(x, t)
$$

may be written as the integral:

$$
x(t)=x_{0}+\int_{t_{0}}^{t} a(x(\tau), \tau) d \tau
$$

Extending this idea, a stochastic differential equation is

$$
\frac{d X}{d t}=a(X, t)+b(X, t) \xi(t)
$$


which may be written by substituting the white noise as

$$
d X(t)=a(X, t) d t+b(X, t) d W(t)
$$

The solution may be expressed as the stochastic integral,

$$
X(t)=X_{0}+\int_{t_{0}}^{t} a[X(\tau), \tau] d \tau+\int_{t_{0}}^{t} b[X(\tau), \tau] d W(\tau)
$$

The first integral is assumed to exist in the mean-square sense. The second integral, in which the integration takes place along the sample path of the Wiener process (insteaid of the time axis) between $W\left(t_{0}\right)$ and $W(t)$, is called the "stochastic integral". It does not have a unique definition, but two different interpretations of the stochastic integrall, one by Ito and the other by Stratonovich, are found in the literature.

\section{Ito stochastic calculus}

Let the interval $\left[t_{0}, t\right]$ in eq $(5.22)$ be divided into $n$ sub-intervals such that

$$
t_{0} \leq t_{1} \leq t_{2} \ldots \leq t_{n-1} \leq t=t_{n}
$$

The Ito stochastic integral is defined as the mean-square limit of the partial sums (Gardiner, 1985):

$$
S_{n}=\sum_{i=1}^{n-1} b\left(x_{i}, t_{i}\right) \Delta W_{i}
$$

where $x_{i}$ is evaluated at $t_{i}$ and $\Delta W_{i}=W_{i+1}-W_{i}$. This way, the stochastic process $X(t)$ is evaluated at the initial point of each sub-interval, and is statistically independent of $\Delta W_{i}$. Thus, if $b$ is a non-anticipating function, the expectation of the Ito stochastic integral vanishes.

The Ito stochastic differential equation (5.21) is equivalent to a one-dimensional diffusion process whose drift term is $a(x, t)$ and diffusion term is $b^{2}(x, t)$ (Gardiner, 1985, p.97). However, due to the interpretation of Ito integral as the mean-square limit of the type described above, it has properties quite different from those of ordinary calculus, which need to be borne in mind while solving or transforming Ito SDE's:

1. $d W(t)^{2}=d t$ $d W(t)^{n+2}=0, n>0$.

i.e., for a non-anticipating random function $G(t)$,

$$
\int_{t_{0}}^{t} G(\tau)[d W(\tau)]^{n+2}= \begin{cases}\int_{t_{0}}^{t} G(\tau) d \tau ; & n=0 \\ 0 & n>0\end{cases}
$$

2. $\int_{t_{0}}^{t} G(\tau) d W(\tau) d \tau=0$ 
3. $\quad d f[W(t), t]=\left(\frac{\partial f}{\partial t}+\frac{1}{2} \frac{\partial^{2} f}{\partial W^{2}}\right) d t+\frac{\partial f}{\partial W} d W(t)$

4. $\int_{t_{1}}^{t_{2}} W(s) d W(s)=W\left(t_{2}\right)^{2}-W\left(t_{1}\right)^{2}-\frac{1}{2}\left(t_{2}-t_{1}\right)$

5. If $X(t)$ obeys equation (5.21), then an arbitrary function, $f[X(t)]$, satisfies the following stochastic differential equation:

$$
d f[X(t)]=\left(a f^{\prime}+\frac{1}{2} b^{2} f^{\prime \prime}\right) d t+b f^{\prime} d W
$$

Thus, a change of variables in Ito stochastic calculus does not give the same results as in ordinary calculus, unless $f(X)$ is a linear function (i.e., $f^{\prime \prime}=0$ ).

\section{Stratonovich stochastic calculus}

For the same subintervals on the time axis as in eq (5.23) the Stratonovich stochastic integral is defined as the mean square limit of a different sequence of partial sums (Gardiner, 1985):

$$
S_{n}=\sum_{i=1}^{n-1} b\left(\frac{1}{2}\left(x_{i}+x_{i+1}\right), t_{i}\right)\left(W_{i+1}-W_{i}\right)
$$

This particular way of defining the partial sum ensures that all the rules of ordinary differential calculus (Reimann integration, change of variables, etc.) remain valid in Stratonovich stochastic calculus.

Ito and Stratonovich solutions of the same stochastic differential equation generally are different. For example, the stochastic integral

$$
\int_{t_{0}}^{t_{1}} W(t) d W(t)= \begin{cases}\frac{1}{2}\left(W\left(t_{1}\right)^{2}-W\left(t_{0}\right)^{2}\right)-\frac{1}{2}\left(t_{1}-t_{0}\right), & \text { using Ito calculus } \\ \frac{1}{2}\left(W\left(t_{1}\right)^{2}-W\left(t_{0}\right)^{2}\right), & \text { using Stratonovich calculus }\end{cases}
$$

However, the Ito solution of an SDE can be shown to be identical to the Stratonovich solution of the same SDE modified in a certain way (Gardiner, 1985). To elaborate, the Ito solution of

$$
d x=a d t+b d W
$$

is same as the Stratonovich solution of

$$
d x=\left[a-\frac{1}{2} b b_{x}\right] d t+b d W
$$

and, the Stratonovich solution of

$$
d x=\alpha d t+\beta d W
$$

is same as the Ito solution of

$$
d x=\left[\alpha+\frac{1}{2} \beta \beta_{x}\right] d t+\beta d W
$$


where the subscript ${ }_{x}$ implies partial derivative with respect to $x$. If the diffusion term of an SDE is independent of $x$, then its Ito and Stratonovich solutions are identical.

In evaluating the relative merits of the Ito and Stratonovich approaches, it is irnportant to note that neither is inherently superior to the other. The choice of one over the other while solving an SDE is guided by computational expediency (Kloeden and Platen, 1992). However once the choice is made, the solution and inferences drawn from it should be internally consistent. Both approaches will be utilized in the sequel.

\subsection{Development of CDM-based Stochastic Models}

\subsubsection{Review of Previous Work}

The amount of published research on stochastic CDM is small compared to the work available involving purely deterministic models, for at least two reasons. First, CDMbased approaches are relatively new in modeling damage growth. Second, experimental data on random damage growth are scarce, which hinders the validation of proposed stochastic models.

Carmeliet and Hens (1994) used a non-random kinetic equation of damage growth. They introduced randomness in the formulation by modeling the initial damage threshold and the ultimate strain as a bivariate Nataf-type random field distributed over the material, and finally employed a finite element analysis for solution.

Woo and $\mathrm{Li}(1992)$ defined damage as a stochastic process and have given suggestions about how to solve the stochastic differential equation that results from damage growth laws. In this they have made use of a dissipation function similar to that discussed in section 3.3. Experimenting on 45 specimens of 2024-T3 Aluminum, Woo and Li (1993b) demonstrated the statistical nature of ductile damage growth. They obtained the first four moments (mean, standard deviation, skewness and kurtosis) of the initial (undamaged) values of $\sigma_{y}, E$ and the Poisson ratios $\nu_{12}$ and $\nu_{13}$. As the test progressed, they computed the mean and standard deviation of $\tilde{E}$ and $\tilde{\nu}_{12}$ at strain values $1 \%, 2 \%$, $\ldots, 25 \%$. However, they did not compute the autocovariance function of $\tilde{E}$ or $\tilde{\nu}_{12}$ which would have led to a more complete characterization of the randomness in the damage growth process. An interesting observation is the extremely high coefficient of variation that they reported for the threshold strain: $V_{\epsilon_{0}}=1.05$.

Harlow and Delph (1995) adopted the familiar power law model for steady statie creep,

$$
\dot{\epsilon}_{\mathrm{c}}=C \sigma^{N}
$$

and the Kachanov model for creep damage growth,

$$
\dot{D}=\frac{A \sigma^{N}}{(1-D)^{N}}
$$


They assumed that $C, A, N$ were time-independent jointly distributed random variables with $C$ and $A$ having the lognormal and $N$ having the normal as their marginal distributions. Spatial variability in the three parameters was excluded, and the formulation did not recognize the possibility of noise in the damage growth process. Analysing high temperature creep rupture test data on AISI 316 stainless steel from Garofalo et al (1961), they computed the means, standard deviations and correlation coefficients of the jointly distributed random variables, $C, A$ and $N$. Finally, using a finite element formulation, they numerically obtained the marginal probability density function of damage at different values of time. The scatter was seen to increase with increasing exposure times for a given value of the applied stress.

Existing CDM-based formulations of stochastic damage growth start with one of the two approaches, as do their deterministic counterparts: (i) a kinetic equation of damage growth, or (ii) a dissipation potential model. Consequently, shortcomings of these methods remain in the stochastic context.

\subsubsection{Stochastic Isotropic Damage Growth}

For a system in diathermal contact with a heat reservoir, the Helmholtz free energy, $\Psi(\theta, \mathbf{x}, D)$, in the deterministic formulation was expressed in terms of the dissipation rate, $\Gamma$, as (cf eq 3.5 )

$$
\Psi=\int\left(\dot{W}-\dot{K}_{E}\right) d t-\int \Gamma d t
$$

where $W$ is the work done on the system and $K_{E}$ is its kinetic energy.

The near-equilibrium deformable body and the heat reservoir in the deterministic formulation undergo rapid and continuous transitions among their microstates. This causes random fluctuation in their internal energies. Owing to the randomness in the fluctuation, the evolution of the free energy can be described as a stochastic process:

$$
\Psi\left(t_{2}\right)=\Psi\left(t_{1}\right)+\int_{t_{1}}^{t_{2}}\left(\dot{W}-\dot{K}_{E}\right) d t-\int_{t_{1}}^{t_{2}} \Gamma d t+\int_{t_{1}}^{t_{2}} \dot{B}(t) d t
$$

where $W, K_{E}$ and $\Gamma$ are the same quantities as described in the deterministic formulation, and $B(t)$ is a zero-mean process (obtained by integrating $\dot{B}(t)$ in the mean-square sense), which is assumed to represent the stochastic contributions to the free energy from the microstructural variations, void interactions and thermal fluctuations described in sec 5.1. If the randomness occurs solely due to thermal fluctuations, then the variance of $B(t)$ may be estimated from statistical mechanics as $\sigma_{B}^{2}(t)=k_{B} \theta^{2} N c_{v}$ (Callen, 1988), where $k_{B}$ is the Boltzmann constant, $\theta$ is the absolute temperature, $N$ is the number of particles and $c_{v}$ is the specific heat at constant volume. In a more elaborate formulation, $B(t)$ may be decomposed into a sum of several random processes, possibly correlated, each of which represents the stochastic contribution from specific sources. However, in the absence of detailed information, all the randomness is lumped into a single process, $B(t)$, which is sufficient for modeling damage growth from a macroscopic viewpoint. 
Proceeding as in the deterministic formulation (sec. 3.5), we can express the stationary variation in the Helmholtz free energy (eq 5.36) as

$$
\begin{aligned}
\delta \Psi\left(t_{2}\right) & =\delta \int_{t_{1}}^{t_{2}}\left(\dot{W}-\dot{K}_{E}+\frac{\partial \Psi}{\partial D} \dot{D}\right) d t-\delta \int_{t_{1}}^{t_{2}}\left(\dot{W}-\dot{K}_{E}-\frac{\partial \Psi}{\partial \mathbf{x}} \dot{\mathbf{x}}\right) d t+\delta \int_{t_{1}}^{t_{2}} \dot{B} d t \\
& =0
\end{aligned}
$$

where we continue with our assumption of isotropic damage. For a deformable body sufficiently close to equilibrium, $\mathbf{x}$ is the symmetric strain tensor and the second term in eq (5.37) has been shown to vanish due to appropriate variations to the velocity field. Consequently, we are left with

$$
\delta\left[\int_{t_{1}}^{t_{2}}\left(\dot{W}-\dot{K}_{E}+\frac{\partial \Psi}{\partial D} \dot{D}+\dot{B}\right) d t\right]=0
$$

Using the commutability of differentiation and variation, we can express the variation $\delta \dot{B}(t)$ on a sample-path basis as

$$
\delta \dot{B}=\frac{d}{d t}(\delta B)=\frac{d}{d t}\left(\frac{\partial B}{\partial \epsilon_{i j}} \delta \epsilon_{i j}\right)
$$

where $B(t)$ is assumed to be a function of strain. Let $b(t)$ denote the fluctuation in the free energy per unit volume,

$$
b(t)=\frac{\partial B(t)}{\partial V}
$$

Continuing as in section 3.5 , we arrive at the condition,

$$
\delta\left(\dot{W}-\dot{K}_{E}+\frac{\partial \Psi}{\partial D} \dot{D}+\dot{B}(t)\right)=0
$$

which leads to the partial differential equations

$$
\begin{gathered}
F_{i}-\rho a_{i}-\left(\psi_{D} D_{i j}^{\prime}+s_{b_{i j}}\right)_{, j}=0 \text { on } \mathcal{R} \\
T_{i}+\left(\psi_{D} D_{i j}^{\prime}+s_{b_{i j}}\right) n_{j}=0 \text { on } \partial \mathcal{R}_{1}
\end{gathered}
$$

where $F_{i}$ are the body forces $(i=1,2,3), T_{i}$ are the surface tractions, $n_{j}$ are the unit outward normals from the surface, $\rho$ is the density, $a_{i}$ are the accelerations at a point, $\psi_{D}=\partial \psi / \partial D, D_{i j}^{\prime}=\partial D / \partial \epsilon_{i j}$ and $s_{b_{i j}}=\partial b / \partial \epsilon_{i j}$. Under uniaxial loading eq (5.43) simplifies to:

$$
\sigma_{\infty}+\psi_{D} \frac{d D}{d \epsilon}+s_{b}=0
$$

where $\sigma_{\infty}$ is the far-field load and $s_{b}=d b / d \epsilon$. Since $b$ is the energy fluctuation per unit volume, $s_{b}$ is the rate of change of this fluctuation caused by straining the deformable body, $\mathcal{R}$. It is noted that $s_{b}$ has dimension of energy per unit volume or, equivalently, 
of stress, and may be interpreted as a random fluctuation imposed on top of the deterministic stress-field existing in $\mathcal{R}$ and on its boundary $\partial \mathcal{R}$. The fluctuating stress $s_{b}$, is therefore a stochastic process indexed with strain, $\epsilon$.

Let us now postulate the nature of this fluctuating stress. First, it makes intuitive sense that $s_{b}(\epsilon)$ is a zero mean process and that the fluctuations assume negative and positive values with equal probability. Second, let us suppose that the mean-square of the fluctuation is the same at all times (or strains). Lastly, let us suppose that the source of the fluctuation is a rapidly varying quantity which may be modelled by a white noise, $\xi(\epsilon)$. Then if $s_{b}$ follows the Langevin equation of the type:

$$
\frac{d s_{b}}{d \epsilon}=-c_{1} s_{b}+\sqrt{c_{2}} \xi
$$

all three assumptions made about $s_{b}$ above can be satisfied by a suitable choice of the positive constants $c_{1}$ and $c_{2}$. The solution of eq (5.45) is the Ornstein-Uhlenbeck process (a Gaussian process), the mean, variance and covariance functions of which are, respectively,

$$
\begin{aligned}
\mathrm{E}\left[s_{b}(\epsilon)\right] & =\mu_{0} e^{-c_{1} \epsilon} \\
\operatorname{var}\left[s_{b}(\epsilon)\right] & =\left[\sigma_{0}^{2}-\frac{c_{2}}{2 c_{1}}\right] e^{-2 c_{1} \epsilon}+\frac{c_{2}}{2 c_{1}} \\
\operatorname{cov}\left[s_{b}\left(\epsilon_{2}\right), s_{b}\left(\epsilon_{1}\right)\right] & =\left[\sigma_{0}^{2}-\frac{c_{2}}{2 c_{1}}\right] e^{-c_{1}\left(\epsilon_{1}+\epsilon_{2}\right)}+\frac{c_{2}}{2 c_{1}} e^{-c_{1}\left|\epsilon_{2}-\epsilon_{1}\right|}
\end{aligned}
$$

where $\mu_{0}$ and $\sigma_{0}^{2}$ are, respectively, the initial mean and variance. For a sufficiently large value of $c_{1}$, the process becomes zero-mean and stationary.

Equations (5.44) and (5.45) describe processes that occur at widely different scales of time (or strain). A large number of fluctuations in $s_{b}$ occur within a time span (or strain increment) which is too small for damage to increase appreciably. Therefore, at a time (or strain) scale that is significant for observing damage, $s_{b}(\epsilon)$ may be approximated by a white noise, $\left(\sqrt{c_{2}} / c_{1}\right) \xi(\epsilon)$ (Gardiner, 1985, chap. 6), giving:

$$
\frac{d D}{d \epsilon}=-\frac{\sigma_{\infty}}{\psi_{D}}-\frac{\sqrt{c_{2}} / c_{1}}{\psi_{D}} \xi(\epsilon)
$$

or,

$$
d D(\epsilon)=-\frac{\sigma_{\infty}}{\psi_{D}} d \epsilon-\frac{\sqrt{c_{2}} / c_{1}}{\psi_{D}} d W(\epsilon)
$$

where $W(\epsilon)$ is the standard Wiener process. The corresponding solution is

$$
D(\epsilon)=D_{0}-\int_{\epsilon_{0}}^{\epsilon} \frac{\sigma_{\infty}}{\psi_{D}} d \epsilon^{\prime}-\int_{\epsilon_{0}}^{\epsilon} \frac{\sqrt{c_{2}} / c_{1}}{\psi_{D}} d W\left(\epsilon^{\prime}\right)
$$

where $D_{0}$ is the initial damage.

Alternately, if the strain rate is known, damage can be expressed as a stochastic process indexed with time:

$$
\frac{d D}{d t}=-\frac{\sigma_{\infty}}{\psi_{D}} \dot{\epsilon}(D, t)-\frac{\dot{\epsilon}(D, t)}{\psi_{D}} s_{b}
$$


where $\dot{\epsilon}$ is the strain rate which is generally a function of time as well as damage. Arguirig as before, $s_{b}$ in this case follows the Langevin equation:

$$
\frac{d s_{b}}{d t}=-c_{3} s_{b}(t)+\sqrt{c_{4}} \xi(t)
$$

where $c_{3}, c_{4}$ are appropriate constants and $\xi(t)$ is the white noise in the time domain. This leads to the following stochastic differential equation of damage growth in time:

$$
d D(t)=-\frac{\sigma_{\infty}}{\psi_{D}} \dot{\epsilon} d t-\frac{\sqrt{c_{4}} / c_{3}}{\psi_{D}} \dot{\epsilon} d W(t)
$$

where $W(t)$ is the standard Wiener process. The solution is

$$
D(t)=D_{0}-\int_{t_{0}}^{t} \frac{\sigma_{\infty}}{\psi_{D}} \dot{\epsilon} d t^{\prime}-\int_{t_{0}}^{t} \frac{\sqrt{c_{4}} / c_{3}}{\psi_{D}} \dot{\epsilon} d W\left(t^{\prime}\right)
$$

where $D_{0}$ is once again the initial damage.

Equation (5.50), or equivalently eq (5.54), is the basic stochastic differential equation (SDE) of damage growth and will be used in the following subsections for ductile, creej and fatigue damage.

It should be recalled here that damage growth is physically an irreversible process in the absence of corrective human intervention. Though the above formulation of stochastic damage growth does not exclude the possibility of instantaneous and local retardations in damage, and though such transient fluctuations may actually occur at the microscale, the damage variable (which deals with the aggregate effect of microscopic processes) should be non-decreasing over a macroscopic interval of time and space. This property of $D(t)$ in the present formulation depends on the relative magnitudes of the drift and diffusion coefficients in eqs (5.50) and (5.54), which should be verified in each case that: they are applied.

The initial value of damage, $D_{0}$, is in general a random variable. It represents the effects of residual stresses, surface roughness, previous loading histories etc - i.e., any deviation from the ideal smooth and virgin structure whose state of damage is precisely equal to zero. Failure ${ }^{4}$ occurs when damage exceeds the critical damage, $D_{c}$ :

$$
\text { Failure : } D(t)>D_{c}, \quad t \in\left[0, t_{L}\right]
$$

The critical damage is assumed to be a material property (section 4.1), which is not necessarily deterministic. Like any other material property, it possesses scatter - partly due to measurement error, and partly due to material variability. The cumulative failure probability at time $t$,

$$
F_{T}(t)=1-L(t)=\mathrm{P}[T \leq t]
$$

\footnotetext{
${ }^{4}$ As explained in section 3.2 and highlighted in chapter 4, failure in the context of CDM is not necessarily fracture, but denotes a stage when the basic assumptions of CDM break down, e.g., with the development of a macro-crack.
} 
where $T$ is the time to failure and $L(t)$ is the reliability function, can be simplified as

$$
F_{T}(t)=\mathrm{P}\left[D(\tau)>D_{c}, \tau \in[0, t]\right] \simeq \mathrm{P}\left[D(t)>D_{c}\right]
$$

provided that $D(t)$ is almost always a non-decreasing function. It will be shown subsequently that it is usually easier to determine the probability structure of the stochastic process $D(t)$ and the cumulative failure probability, $F_{T}(t)$, by first treating the material properties as deterministic (in the drift and diffusion coefficients). However, $D_{c}$ and the other material properties entering the damage growth equations (e.g., $E, K, \sigma_{f}, S_{e}$ ) are generally random and may possess a fair amount of stochastic dependence, due to a common origin at the microstructural level. If this dependence is known, the conditioning on $F_{T}(t)$ may be removed by using the theorem of total probability, and sensitivity studies can be conducted. The randomness in only the significant parameters can be retained, reducing the dimensionality of the problem.

In the following three sections, the above formulation will be applied to ductile deformation, creep and fatigue damage. The predictions of the proposed models will be compared with available experimental data to determine how well they can capture the various aspects of random damage growth processes. Simple reliability computations will also be presented as a precursor to their later use in more complex situations in Chapter 6.

\subsection{Stochastic Ductile Damage}

\subsubsection{Equation and Stochastic Characterization}

The mechanistic bases of stochastic ductile deformation damage are considered to remain the same as in the deterministic formulation. The expressions for $\sigma_{\infty}$ and $\psi_{D}$ in eq (5.50) above can therefore be used directly from sec. 4.2 .

For ductile plastic damage under uniaxial monotonic loading, the SDE governing the strain-rate of damage growth (eq 5.50) can be written as

$$
d D\left(\epsilon_{p}\right)=A\left(\epsilon_{p}\right)\left(1-D\left(\epsilon_{p}\right)\right) d \epsilon_{p}+B\left(\epsilon_{p}\right) d W\left(\epsilon_{p}\right)
$$

where $\epsilon_{p}$ is the plastic strain. It is convenient to make the substitution $X\left(\epsilon_{p}\right)=1-D\left(\epsilon_{p}\right)$, and write instead:

$$
d X\left(\epsilon_{p}\right)=-A\left(\epsilon_{p}\right) X\left(\epsilon_{p}\right) d \epsilon_{p}-B\left(\epsilon_{p}\right) d W\left(\epsilon_{p}\right)
$$

The coefficients are:

$$
A\left(\epsilon_{p}\right)=\frac{\epsilon_{p}^{\frac{1}{M}}}{\frac{\epsilon_{p}^{1+\frac{1}{M}}}{1+\frac{1}{M}}+C}
$$




$$
B\left(\epsilon_{p}\right)=\frac{\left(\sqrt{c_{2}} / c_{1}\right) / K}{\frac{\epsilon_{p}^{1+\frac{1}{M}}}{1+\frac{1}{M}}+C}
$$

where the constant

$$
C=\frac{3}{4} \frac{\sigma_{f}}{K}-\frac{\epsilon_{0}^{1+\frac{1}{M}}}{1+\frac{1}{M}}
$$

and $\epsilon_{0}$ is the threshold plastic strain for damage initiation, $M$ is the strain hardening exponent, $K$ is the hardening modulus, $E$ is the elastic modulus and $\sigma_{f}$ is the local failure stress. $E$ is two to three orders of magnitude larger than $K$, making $K /(2 E) \sim 0$, and allowing the coefficients to be simplified as before.

The drift term, $B\left(\epsilon_{p}\right)$, of eq (5.60) is independent of damage which, as may be recalled from sec. 5.2, causes the Ito and Stratonovich solutions to be identical. It thus is unnecessary to choose one approach over the other, and the rules of ordinary calculus can be used to arrive at a closed form solution. Let us introduce a new stochastic process, $Y(\epsilon)$ as:

$$
Y=X\left(\frac{\epsilon^{1+\frac{1}{M}}}{1+\frac{1}{M}}+C\right)
$$

where $\epsilon$ is the plastic strain. From this point on in this section, we will drop the subscript $p$ in plastic strain to improve readability. Differentiating $Y$,

$$
\begin{aligned}
d Y & =d X\left(\frac{\epsilon^{1+\frac{1}{M}}}{1+\frac{1}{M}}+C\right)+X \epsilon^{1 / M} d \epsilon \\
& =\left[\frac{\epsilon^{1+\frac{1}{M}}}{1+\frac{1}{M}}+C\right][-X A(\epsilon) d \epsilon-B(\epsilon) d W(\epsilon)]+X \epsilon^{1 / M} d \epsilon \\
& =\frac{\epsilon^{1 / M}}{A(\epsilon)}[-X A(\epsilon) d \epsilon-B(\epsilon) d W(\epsilon)]+X \epsilon^{1 / M} d \epsilon \\
& =-\frac{B(\epsilon)}{A(\epsilon)} \epsilon^{1 / M} d W(\epsilon) \\
& =-\frac{\left(\sqrt{c_{2}} / c_{1}\right)}{K} d W(\epsilon)
\end{aligned}
$$

The solution of $Y(\epsilon)$ is now straightforward:

$$
\begin{aligned}
Y(\epsilon) & =Y\left(\epsilon_{0}\right)-\frac{\left(\sqrt{c_{2}} / c_{1}\right)}{K} \int_{\epsilon_{0}}^{\epsilon} d W\left(\epsilon^{\prime}\right) \\
& =Y\left(\epsilon_{0}\right)-\frac{\left(\sqrt{c_{2}} / c_{1}\right)}{K}\left[W(\epsilon)-W\left(\epsilon_{0}\right)\right]
\end{aligned}
$$


Substituting eq (5.64), the solution for $X$ is:

$$
X(\epsilon)=X\left(\epsilon_{0}\right) \frac{\epsilon_{0}^{1+\frac{1}{M}}+\left(1+\frac{1}{M}\right) C}{\epsilon^{1+\frac{1}{M}}+\left(1+\frac{1}{M}\right) C}-\frac{\left(\sqrt{c_{2}} / c_{1}\right) / K}{\frac{\epsilon^{1+\frac{1}{M}}}{1+\frac{1}{M}}+C}\left[W(\epsilon)-W\left(\epsilon_{0}\right)\right]
$$

Finally, the solution in terms of $D(\epsilon)=1-X(\epsilon)$ is

$$
\begin{aligned}
D(\epsilon) & =1-\left(1-D\left(\epsilon_{0}\right)\right) \frac{\epsilon_{0}^{1+\frac{1}{M}}+\left(1+\frac{1}{M}\right) C}{\epsilon^{1+\frac{1}{M}}+\left(1+\frac{1}{M}\right) C}+\frac{\left(\sqrt{c_{2}} / c_{1}\right) / K}{\frac{\epsilon^{1+\frac{1}{M}}}{1+\frac{1}{M}}+C}\left[W(\epsilon)-W\left(\epsilon_{0}\right)\right] \\
& =1-\left(1-D\left(\epsilon_{0}\right)\right) \frac{(3 / 4)\left(\sigma_{f} / K\right)\left(1+\frac{1}{M}\right)}{\epsilon^{1+\frac{1}{M}}+\left(1+\frac{1}{M}\right) C}+\frac{\left(\sqrt{c_{2}} / c_{1}\right) / K}{\frac{\epsilon^{1+\frac{1}{M}}}{1+\frac{1}{M}}+C}\left[W(\epsilon)-W\left(\epsilon_{0}\right)\right]
\end{aligned}
$$

$D\left(\epsilon_{0}\right)=D_{0}$ being the initial damage. Unless otherwise stated, the material properties $\underline{\Omega}=\left\{\epsilon_{0}, \sigma_{f}, K, M\right\}$ are considered non-random in the following.

The mean of the process is

$$
\mu_{D \mid \underline{\Omega}}(\epsilon)=1-\left(1-\mu_{D_{0}}\right) \frac{(3 / 4)\left(\sigma_{f} / K\right)}{\frac{\epsilon^{1+\frac{1}{M}}}{1+\frac{1}{M}}+C}
$$

which is identical to the solution in the deterministic formulation (eq 4.36) with $\mu_{D_{0}}=0$. The variance is

$$
\sigma_{D \mid \underline{\Omega}}^{2}(\epsilon)=\sigma_{D_{0}}^{2}\left(\frac{(3 / 4)\left(\sigma_{f} / K\right)}{\frac{\epsilon^{1+\frac{1}{M}}}{1+\frac{1}{M}}+C}\right)^{2}+\left(\epsilon-\epsilon_{0}\right)\left(\frac{\left(\sqrt{c_{2}} / c_{1}\right) / K}{\frac{\epsilon^{1+\frac{1}{M}}}{1+\frac{1}{M}}+C}\right)^{2}
$$

where $\sigma_{D_{0}}^{2}$ is the initial variance, which is equal to zero if the initial condition is deterministic. Finally, the autocovariance function of the process, for $\epsilon_{2} \geq \epsilon_{1} \geq \epsilon_{0}$, is

$$
\begin{aligned}
\operatorname{cov}\left[D\left(\epsilon_{1}\right), D\left(\epsilon_{2}\right) \mid \Omega\right]= & \sigma_{D_{0}}^{2}\left(\frac{(3 / 4)\left(\sigma_{f} / K\right)}{\frac{\epsilon_{1}^{1+\frac{1}{M}}}{1+\frac{1}{M}}+C}\right)\left(\frac{(3 / 4)\left(\sigma_{f} / K\right)}{\frac{\epsilon_{2}}{1+\frac{1}{M}}+C}\right) \\
& +\left(\epsilon_{1}-\epsilon_{0}\right)\left(\frac{\left(\sqrt{c_{2}} / c_{1}\right) / K}{\frac{1}{M}}\right)\left(\frac{\left(\sqrt{c_{2}} / c_{1}\right) / K}{\frac{\epsilon_{1}}{1+\frac{1}{M}}+C}\right)\left(\frac{\epsilon_{2}^{1+\frac{1}{M}}}{1+\frac{1}{M}}+C\right)
\end{aligned}
$$


which reduces to $\sigma_{D \mid \underline{\Omega}}^{2}(\epsilon)$ when $\epsilon=\epsilon_{1}=\epsilon_{2}$. It is clearly seen that the ductile damage growth is a non-stationary process. If the initial value of damage, $D\left(\epsilon_{0}\right)$, is either Gaussian or deterministic, then $D(\epsilon)$ itself is a Gaussian stochastic process (since the Wiener process in eq 5.68 is Gaussian). This notion is consistent with the definition of the damage variable: assuming that the random sizes of the numerous microscopic voids giving rise to damage are statistically independent of each other, then, by its definition, damage can be shown to approach in distribution a normal variable when the number of defects is large.

If the joint probability density of $\underline{\Omega}$ is known, then the unconditional mean and variance of the damage process may be obtained as,

$$
\begin{aligned}
& \mu_{D}(\epsilon)=\int \ldots \int \mu_{D \mid \underline{\Omega}}(\epsilon) f_{\underline{\Omega}}(\underline{\omega}) d \underline{\omega} \\
& \sigma_{D}^{2}(\epsilon)=\int \ldots \int \sigma_{D \mid \underline{\Omega}}^{2}(\epsilon) f_{\underline{\Omega}}(\underline{\omega}) d \underline{\omega}
\end{aligned}
$$

using the theorem of total probability. $D(\epsilon)$ is generally non-Gaussian in this case and the unconditional distribution and covariance structure of $D(\epsilon)$ may be obtained numerically.

\subsubsection{Failure Probability in Random Ductile Damage Growth}

Failure occurs when damage reaches the critical value $D_{c}$, which may be less than or equal to 1 . The cumulative probability of failure at strain $\epsilon, F_{\epsilon_{f}}(\epsilon)$, which is the complement of the reliability function, $L(\epsilon)$, is

$$
\begin{aligned}
F_{\epsilon_{f}}(\epsilon) & =1-L(\epsilon) \\
& =\mathbf{P}\left[\epsilon_{f} \leq \epsilon\right] \\
& =1-P\left[D\left(\epsilon^{\prime}\right) \leq D_{c} ; \forall \epsilon^{\prime} \in[0, \epsilon]\right]
\end{aligned}
$$

where $\epsilon_{f}$ is the failure strain. Mathematically, this is a first passage problem.

Although damage growth is an irreversible process (autogenous healing is ruled out), the mathematical form of the damage growth SDE (5.60) allows the possibility of negative damage increment. The probability of a negative damage increment depends on the drift and diffusion terms and also on the time (or strain) interval of observation. If the drift in damage growth is large compared to its diffusion, then the growth rate is almost always positive. Under this condition, the sample paths of $D(\epsilon)$ which cross $D_{c}$ (from below) for the first time at $\epsilon_{1}<\epsilon$, may be expected to stay above that barrier at $\epsilon$. This becomes more and more likely the larger the interval $\left(\epsilon-\epsilon_{1}\right)$ gets. In such cases, the reliability can be simplified as the CDF of the damage function evaluated at the critical damage:

$$
F_{\epsilon_{f}}(\epsilon) \simeq 1-P\left[D(\epsilon) \leq D_{c}\right]
$$


The validity of this assumption should be established in every case that it is adopted. The increment in damage, $\Delta D(\epsilon ; \Delta \epsilon)$, over an interval of plastic strain, $[\epsilon, \epsilon+\Delta \epsilon]$, is a random quantity. Its mean and variance are:

$$
\begin{aligned}
\mathrm{E}[\Delta D(\epsilon ; \Delta \epsilon)] & =A(\epsilon)\left(1-\mu_{D}(\epsilon)\right) \Delta \epsilon \\
\operatorname{var}[\Delta D(\epsilon ; \Delta \epsilon)] & =A(\epsilon)^{2} \Delta \epsilon^{2} \sigma_{D}(\epsilon)^{2}+B(\epsilon)^{2} \Delta \epsilon
\end{aligned}
$$

where $A$ and $B$ are given by eqs(5.61) and (5.62). The probability of negative damage increment over the range $\Delta \epsilon$,

$$
P[\Delta D(\epsilon ; \Delta \epsilon) \leq 0]
$$

is a function of the position as well as the length of the interval.

Fixing the initial condition and the material parameters, $\underline{\Omega}_{1}=\left\{D_{0}, \epsilon_{0}, \sigma_{f}, K, M, D_{c}\right\}$, the cumulative failure probability and the probability of negative damage increment under assumption (5.75), can be written, respectively, as:

$$
F_{\epsilon_{f} \mid \underline{\Omega}_{1}}(\epsilon)=1-\Phi\left(\frac{D_{c}-\mu_{D \mid \underline{\Omega}_{1}}(\epsilon)}{\sigma_{D \mid \underline{\Omega}_{1}}(\epsilon)}\right)
$$

and

$$
P\left[\Delta D(\epsilon ; \Delta \epsilon) \leq 0 \mid \underline{\Omega}_{1}\right]=\Phi\left[\frac{-A\left(1-\mu_{D \mid \underline{\Omega}_{1}}\right)}{\sqrt{A^{2} \sigma_{D \mid \underline{\Omega}_{1}}^{2}+B^{2} /(\Delta \epsilon)}}\right]
$$

where $\mu_{D \mid \underline{\Omega}_{1}}(\epsilon)$ and $\sigma_{D \mid \underline{\Omega}_{1}}(\epsilon)$ may be obtained by setting $\mu_{D_{0}}=D_{0}$ and $\sigma_{D_{0}}=0$ in eqs (5.69) and (5.70) respectively, and $\Phi(\cdot)$ is the standard normal cumulative distribution function. The theorem of total probability may be used to remove the conditioning on $\underline{\Omega}_{1}$ if their joint probability structure is known.

\subsubsection{Model Validation, Sensitivity and Reliability Studies}

The deterministic version of the above stochastic ductile damage growth model has been shown to match experimental results and observed trends in material behavior. The proposed approach to stochastic damage growth, unlike many of the existing methods, deals with two different aspects of randomness: one that pertains to the initial conditions and material parameters, and the other that is associated with the instantaneous growth rate of the process (in the form of a stochastic noise). A knowledge of both sources of randomness is required for a satisfactory description of stochastic damage growth. The accuracy of the proposed stochastic model will now be investigated. The rationale of combining the effects of various random factors like microstructural variations, void interactions and thermal fluctuations into one single stochastic process (superposed on the scalar Helmholtz free energy) will also be examined.

Fig. 5.1 shows the predicted mean $\mu_{D}(\epsilon)$ and standard deviation $\sigma_{D}(\epsilon)$ (eqs 5.69 and 5.70) for ductile damage growth in 2024-T3 aluminum with deterministic initial 
conditions and material parameters, and compares them with the experimental results from Woo and $\mathrm{Li}(1993 \mathrm{~b})$. Since this reference does not report the required material parameters (nominal or statistical) of the specimens tested, the nominal values from Table 4.1 are used. The value of $\epsilon_{0}=0.016$ is taken from Lemaitre (1985) and the material is assumed to be initially defect-free ${ }^{5}$. The variance and correlation length of the fluctuating stress used in the experiment is unknown, and a value of $\sqrt{c_{2}} / c_{1}=20 \mathrm{MPa}$ has been assumed ${ }^{6}$. The mean function, which is identical to the deterministic solution (eq 4.36), agrees well with the experimental mean damage from Woo and $\mathrm{Li}$ (1993b). It also compares well with the data from Lemaitre (1985), but no statistical information is available about Lemaitre's data. The predicted standard deviation in damage growth also agrees well with Woo and Li's (1993b) experiment, and in particular, predicts the decelerated growth in the experimental standard deviation correctly.

Figures 5.2 (a) through (d) show the effect of treating $\epsilon_{0}, \sigma_{f}, K$ and $M$ as random one at a time ${ }^{7}$. The noise parameter in the model and the experimental data points are the same as before. The coefficient of variation (c.o.v.) of $\epsilon_{0}$ is taken from Woo and $\mathrm{Li}$ (1993b). The c.o.v.'s of the other three and all statistical distributions are assumed and shown in the figures. It is observed that the mean damage is not affected in any of the four cases. The standard deviation of damage is insensitive to randomness in $\epsilon_{0}$ or $M$, but increases when $\sigma_{f}$ or $K$ is considered random. These findings are consistent with the sensitivity study done on the deterministic model in section 4.2.2.

Figs 5.3 (b) through (d) show the effect of treating all four variables as random ${ }^{8}$ : (b) when they are perfectly correlated, (c) when they are moderately correlated, and (d) when they are statistically independent. Fig 5.3(a) reproduces the case when the variables are treated deterministically. The noise parameter in the model and the experimental data points are the same as before. It is observed that treating the parameters as random and varying the correlation among them has almost no effect on the mean damage. However, the standard deviation of $D(t)$ is significantly affected when all variables are considered random, and is the highest when the random variables are considered. statistically independent. It is therefore important to know the correlation structure of the initial condition and material parameters: considering them as independent may over-estimate the scatter in damage by a factor of 2 .

Figs 5.4 (a) - (d) show the effect of the noise intensity, $\sqrt{c_{2}} / c_{1}$, on the damage growth

\footnotetext{
${ }^{5}$ The initial damage is considered zero $\left(\mu_{D_{0}}=0, \sigma_{D_{0}}=0\right)$ in all cases considered in this subsection for 2024-T3 Aluminum, to conform with the way the experiment was conducted.

${ }^{6}$ Several values of the unknown noise intensity $\sqrt{c_{2}} / c_{1}$ were tried, and their effects on the sample paths of damage growth, holding all parameters deterministic, were observed. Values above $40 M P a$ produced occasional but significant negative damage increments, and consequently the noise parameter was restricted below $40 M P a$.

${ }^{7}$ In this analysis, the mean of a random variable is taken equal to its nominal value.

${ }^{8} \sigma_{f}, K, M$ are assumed normal in this analysis, and $\epsilon_{0}$ is assumed lognormal. The marginal statistics are the same as before. When considered perfectly correlated, the correlation matrix of $\ln \left(\epsilon_{0}\right), \sigma_{f}, K, M$ is the identity matrix. When the random variables are "moderately correlated", the off-diagonal terms in the correlation matrix are all taken to be 0.5 .
} 
process in the presence of moderate correlation $(\rho=0.5)$ among the random variables $\epsilon_{0}, \sigma_{f}, K, M$. Figs 5.5 (a) - (d) repeat the above with perfect correlation $(\rho=1.0)$ among the random variables. In particular, Figs 5.4 (a) and 5.5 (a) pertain to the case when the noise is entirely absent. It is observed that the intensity of the noise has no effect on the mean of the process (as predicted by eq 5.69), but has a significant effect on the standard deviation of damage. Among all the cases considered for 2024-T3 Al, Fig 5.5(b) is seen to match the data (Woo and $\mathrm{Li}, 1993 \mathrm{~b}$ ) closest, however, this kind of agreement could also be achieved with other combinations of the noise intensity, the correlation coefficients and the marginal distributions.

As noted previously, the stochastic model in eq (5.60) admits the possibility of negative damage increment. Fig 5.6 shows the probability of negative damage increment, $\Delta D(\epsilon, \Delta \epsilon)$, over different interval sizes as a function of strain for 2024-T3 Al with the same properties as above. As the interval size, $\Delta \epsilon$, approaches zero, the instantaneous growth rate approaches a $50 \%$ probability of attaining negative values, due to the special nature of the white noise (i.e., infinite variance). However, as $\Delta \epsilon$ increases, the probability rapidly falls off to negligible quantities $\left(\sim 10^{-10}\right.$ for $\left.\Delta \epsilon=0.1\right)$.

Figs 5.7(a) and (b) illustrate the limit state probability for 2024-T3 Al:

$$
\mathrm{P}\left[\epsilon_{f} \leq \epsilon\right] \simeq 1-\mathrm{P}\left[D(\epsilon) \leq D_{c}\right]
$$

in which the failure strain, $\epsilon_{f}$, is now a random variable. In Fig 5.7(a), only $D_{c}$ is treated as random ${ }^{9}$ while the others $\left(\epsilon_{0}, \sigma_{f}, K, M\right)$ are held constant at their nominal values. In Fig 5.7(b), all five parameters are considered random (with marginal distributions and statistics same as before) with correlation coefficient 0.5 between each pair. The noise intensity is $20 \mathrm{MPa}$ in either figure. To give a visual sense of the scatter in the process, a few sample functions of $D(\epsilon)$ (selected at random) are shown in the figures, which were numerically obtained from eq (5.60) using an interval size $\Delta \epsilon=0.01$. No sample function is seen to return to the safe region once it exits that region, reinforcing the notion of non-negative damage growth. The scatter in the sample functions is greater in Fig 5.7(b) as may be expected intuitively. The relation between $D$ and $\epsilon$ is non-linear, involves the Wiener process and cannot be inverted explicitly. The cumulative distribution function (CDF) of $\epsilon_{f}$ is obtained numerically (eq 5.81) using step size $\Delta \epsilon=0.01$, following which, its probability density function (PDF) is obtained by numerical differentiation. The mean and standard deviation of $\epsilon_{f}$ are found to be, respectively, 0.237 and 0.034 in the first case (Fig5.7 (a)) and 0.247 and 0.052 in the second case (Fig5.7 (b)), values which are of the same order as those generally observed for engineering metals. As may be expected, the scatter in $\epsilon_{f}$ increased when all the parameters were considered as random variables.

Different aspects of random ductile damage growth were considered in this subsection. The importance of noise in the process vis a vis variability in initial conditions was investigated and a knowledge of both was found necessary for a satisfactory characterization

\footnotetext{
${ }^{9} D_{c}$ is assumed to be a normal random variable with mean 0.23 (equal to the deterministic quantity from Lemaitre, 1985) and a coefficient of variation 0.10 .
} 
of random damage growth. Dependence among the material parameters was considered and their correlation structure was found to play a significant role in the scatter of the damage growth process. Finally a scheme to perform reliability analyses was outlined.

\subsection{Stochastic Creep Damage}

\subsubsection{Equations and Stochastic Characterization}

The mechanistic basis of stochastic creep damage growth is assumed to remain the same as in the deterministic approach (sec 4.3), eqs (4.44) and (4.45):

$$
\dot{\epsilon}_{c}=\frac{A}{(1-D)^{m}} \phi \sigma_{\infty}^{m} t^{\phi-1}
$$

where $\sigma_{\infty}$ is the far-field applied stress. The partial derivative of the free energy, $\psi_{D}$, was derived in section 4.3 and the derivation will not be repeated here. However, the final expression is reproduced below, as it will be used in the SDE for creep damage growth:

$$
\psi_{D}=-\frac{3}{4} \sigma_{f}
$$

where $\sigma_{f}$ is the true failure stress at the operating temperature.

Under high-temperature constant stress creep, the increment in total strain is the same as that in creep strain, i.e., $\dot{\epsilon}=\dot{\epsilon}_{c}$. The stochastic differential equation of isotropic creep damage growth is therefore,

$$
d D(t)=-\frac{\sigma_{\infty}}{\psi_{D}} \dot{\epsilon}_{c} d t-\frac{\sqrt{c_{4}} / c_{3}}{\psi_{D}} \dot{\epsilon}_{c} d W(t)
$$

Substituting the expression for creep strain rate

$$
d D(t)=\frac{4}{3} \frac{A \phi \sigma_{\infty}^{m+1} t^{\phi-1}}{\sigma_{f}(1-D)^{m}} d t+\left(\sqrt{c_{4}} / c_{3}\right) \frac{4}{3} \frac{A \phi \sigma_{\infty}^{m} t^{\phi-1}}{\sigma_{f}(1-D)^{m}} d W(t)
$$

To the knowledge of the author, eq (5.85) does not have a closed-form solution in Ito calculus. But a closed-form solution is possible in the Stratonovich sense, under the condition $\phi=1$ (steady state creep). Eq (5.85) is rewritten as

$$
(1-D(t))^{m} d D(t)=A_{1} d t+B_{1} d W(t)
$$

where the time-independent coefficients

$$
\begin{aligned}
& A_{1}=\frac{4}{3} \frac{A}{\sigma_{f}} \sigma_{\infty}^{m+1} \\
& B_{1}=\frac{4}{3} \frac{A}{\sigma_{f}} \sigma_{\infty}^{m}\left(\sqrt{c_{4}} / c_{3}\right)
\end{aligned}
$$


It should be noted that $A_{1}$ and $B_{1}$ are generally random variables on account of material variability. The Stratonovich solution of eq $(5.86)$ is

$$
(1-D(t))^{m+1}=\left(1-D_{0}\right)^{m+1}-A_{1} t(m+1)-B_{1}(m+1) W(t)
$$

where the initial time $t_{0}=0$ and the initial damage is $D_{0}$. Rearranging, damage can be expressed as a function of time:

$$
D(t)=1-\left[\left(1-D_{0}\right)^{m+1}-A_{1}(m+1) t\right]^{\frac{1}{1+m}}\left[1-\frac{B_{1}(m+1) W(t)}{\left(1-D_{0}\right)^{m+1}-A_{1} t(m+1)}\right]^{\frac{1}{1+m}}
$$

which is equal to the earlier deterministic solution (eq 4.55) in terms of $(1-D)$ for steady state creep, multiplied with a term containing noise. If the noise vanishes $\left(c_{4}=0\right.$ in eq 5.53, which implies $B_{1}=0$ above), the earlier deterministic solution and the above solution of random creep damage coincide for given values of the material parameters $\left(A, \dot{m}, \sigma_{f}\right)$ and initial damage, $\dot{D}_{0}$.

Damage is bounded in the range [0,1]. Since the Wiener process is Gaussian with mean 0 and variance $t$, the cumulative distribution function (CDF) of $D(t)$, conditioned on fixed values of the initial condition and material properties, $\underline{\Omega}=\left\{D_{0}, A, \sigma_{f}, m\right\}$, is

$$
F_{D \mid \underline{\Omega}}(d ; t)=\mathrm{P}[D(t) \leq d]= \begin{cases}0, & d<0 \\ \Phi\left[\frac{p(t)-(1-d)^{m+1}}{q(t)}\right], & 0<d<1 \\ 1, & d>1\end{cases}
$$

where $\Phi(\cdot)$ is the standard normal distribution function and

$$
\begin{aligned}
& p(t)=\left(1-d_{0}\right)^{m+1}-A_{1} t(m+1) \\
& q(t)=B_{1}(m+1) \sqrt{t}
\end{aligned}
$$

Here, $d_{0}$ is the fixed value of the random variable $D_{0}$. Since the Wiener process can assume values anywhere in $(-\infty, \infty), D(t)$ has a mixed distribution, with finite probabilities at 0 and 1 :

$$
\begin{aligned}
& P_{0}(t)=\mathrm{P}[D(t)=0]=\Phi\left[\frac{p(t)-1}{q(t)}\right] \\
& P_{1}(t)=\mathrm{P}[D(t)=1]=1-\Phi\left[\frac{p(t)}{q(t)}\right]
\end{aligned}
$$

The first two moments of $D(t)$ are

$$
\mathrm{E}[D(t)]=0 \cdot P_{0}(t)+\int_{0}^{1} d \frac{(m+1)(1-d)^{m}}{q(t)} \phi\left[\frac{p(t)-(1-d)^{m+1}}{q(t)}\right] d d+1 \cdot P_{1}(t)
$$




$$
\mathrm{E}\left[D(t)^{2}\right]=0 \cdot P_{0}(t)+\int_{0}^{1} d^{2} \frac{(m+1)(1-d)^{m}}{q(t)} \phi\left[\frac{p(t)-(1-d)^{m+1}}{q(t)}\right] d d+1^{2} \cdot P_{1}(t)
$$

where $\phi(\cdot)$ is the standard normal density function. Higher moments of $D(t)$ can be obtained similarly, if required. If the initial condition and material parameters are random and their joint probability density is known, the unconditional moments and the unconditional CDF should be obtained using the theorem of total probability.

As discussed in detail in sections 3.2 and 5.3.2, creep failure occurs when the damage process exceeds the critical damage, $D_{\mathrm{c}}$. The instantaneous failure probability, conditioned on $\underline{\Omega}_{1}=\left\{\underline{\Omega}, D_{c}\right\}$, is

$$
P_{f \mid \Omega_{1}}(t)=\mathrm{P}\left[D(t)>D_{c}\right]
$$

The conditioning may be removed if the joint density of $\underline{\Omega}_{1}$ is known. Arguing as in the case of stochastic ductile damage, the cumulative failure probability, $F_{T}(t)$, can be approximated as the instantaneous failure probability, $P_{f}(t)$, above, provided that the damage growth rate is almost always positive:

$$
F_{T}(t) \simeq P_{f}(t)=\mathrm{P}\left[D(t)>D_{c}\right]
$$

Since the mathematical form of the SDE governing creep damage growth admits the possibility of negative damage growth (the probability of which depends on the drift; and diffusion coefficients and the length of observation as discussed in section 5.3.2) the validity of the preceding assumption should be established in each case that it is applied.

The SDE which has the same solutions in the Ito sense (using eq 5.32 for the transformation) is

$$
d D=\left[A_{1}(1-D)^{-m}+\frac{1}{2} B_{1}^{2} m(1-D)^{-2 m-1}\right] d t-B_{1}(1-D)^{-m} d W(t)
$$

This equation does not have a closed-form solution, as mentioned at the beginning of this subsection. Numerical estimates of the mean, variance and failure probabilities from. eq (5.100) may still be obtained by simulating sample paths of $D(t)$ using the Euler scheme $\mathrm{e}^{10}$ :

$$
D_{i+1}=D_{i}+\left[A_{1}\left(1-D_{i}\right)^{-m}+\frac{1}{2} B_{1}^{2} m\left(1-D_{i}\right)^{-2 m-1}\right] \Delta t_{i+1}-B_{1}\left(1-D_{i}\right)^{-m} \Delta W_{i+1}
$$

where $\Delta W_{i}$ and $\Delta W_{j}$ are statistically independent for $i \neq j, D_{i}$ and $\Delta W_{i+1}$ are statistically independent $\forall i$.

\subsubsection{Model Verification and Studies in Reliability}

No published CDM-based studies of stochastic creep damage growth (e.g., by measuring the reduced stiffness) could be located that present data on the mean and variance (and

\footnotetext{
${ }^{10}$ The Euler scheme of discretization is computationally feasible only if the Wiener increments are independent of the stochastic process as well as of each other, and this is ensured if the Ito interpretation is used.
} 
possibly higher moments and the correlation structure) of damage as a function of time. There are limited data available on the random time to rupture and the random time to the onset of tertiary creep (e.g., Garofalo et al, 1961). The statistics of the random time to failure predicted by the present model will be compared with such data, but this comparison is less than ideal because it does not give an opportunity to study the behavior of the structure when it is still in service. Thus, predictions of the mean and the standard deviation of creep damage based on published test conditions are presented in the following, primarily to study the various features of the present stochastic model and its agreement with the deterministic model. The predicted cumulative failure probability is also presented to observe the effect of the noise on reliability and to determine whether it can be substituted by the more easily available closed-form solution of instantaneous failure probability. In the following numerical examples, creep damage growth in A36 steel stressed to $12 \mathrm{ksi}(83 \mathrm{MPa})$ at $1000^{\circ} \mathrm{F}\left(538^{\circ} \mathrm{C}\right)$ in type 316 stainless steel stressed to $199 \mathrm{MPa}$ at $1100^{\circ} \mathrm{F}\left(593^{\circ} \mathrm{C}\right)$ is analyzed.

Figs 5.8(a) and (b) show sample paths of $D(t)$ for A36 steel obtained with the help of eq (5.101) (with $\Delta t=12 h r$ ) for two different noise intensities. The material is assumed initially defect-free $\left(\mu_{D_{0}}=\sigma_{D_{0}}=0\right)$, and the material parameters (taken from Table 4.3) are considered deterministic. As may be expected, the scatter in the sample paths of damage is found to increase with a higher noise intensity. Figs 5.9(a) and (b) show the corresponding mean damage, standard deviation of damage and failure ${ }^{11}$ probabilities obtained in two different ways: (i) in closed-form ${ }^{12}$ from the Stratonovich solution, and (ii) by Monte-Carlo simulation using the equivalent Ito SDE (eq 5.101) ${ }^{13}$. The means and standard deviations obtained by the two different methods are found to be almost identical, thus indicating the unbiasedness of the numerical solution. The failure probability obtained in closed-from (using the Stratonovich approach) is found to match the numerical solution (using the Ito simulation) very well, which confirms the accuracy of the approximation (5.99). The closed-form estimate (CDF of $D(t)$ ) may therefore be adopted to determine reliability, leading to a considerable computational advantage, particularly when removing the conditioning on $D_{0}, D_{c}$ or the material parameters. The deterministic solution for the same condition of stress and temperature agrees closely with the predicted mean damage. The standard deviation of damage is found to grow with time until the mean damage is close to 1 after which it starts to decrease, which corroborates the fact that $D=1$ is an absorbing boundary. The scatter in the time to failure increases (the slope of $F_{T}$ becomes flatter) with increasing noise intensity.

The material parameters $\left(A, m, D_{c}\right)$ were treated as deterministic and the initial damage $\left(D_{0}\right)$ was neglected in the above examples. The only source of scatter was the

\footnotetext{
${ }^{11}$ Failure is with respect to a non-random $D_{c}=0.5$

${ }^{12}$ The integrations in eqs (5.96) and (5.97) are performed using the simple trapezoidal rule with a step-size of $\Delta d=0.00001$

${ }^{13} \mathrm{~A}$ constant time-step of $\Delta t=12 \mathrm{hr}$ is used in this Enler scheme of integration. 1 million sample paths are generated for the estimates. The probability of negative damage increment is found to vary between 0 and $4 \times 10^{-5}$ for the lower noise intensity, and for the higher noise intensity, the probability of negative damage increment increases up to a maximum of $2 \%$ for the time step of $12 \mathrm{hrs}$.
} 
noise in the form of the non-zero parameter $\sqrt{c_{4}} / c_{3}$. Furthermore, only the Stratonovich interpretation of the creep damage SDE (5.85), was adopted above. These restrictions are removed in the following examples on creep damage growth in type 316 stainless steel. The material properties and the initial damage ${ }^{14}$ are allowed to be random variables in the following ${ }^{15}$, and the differences in the Ito and Stratonovich interpretations of the same SDE (5.85) of random creep damage growth are illustrated.

Figs $5.10(\mathrm{a})$ and (b) show the effect of treating the material parameters and the initial damage as random variables when the Stratonovich interpretation of SDE (5.85) is adopted. Figs 5.11(a) and (b) repeat the comparison with the Ito interpretation of SDE (5.85). When $A, m$ and $D_{0}$ are considered random, the sample paths of $D(t)$ show significant scatter compared to when only the noise is present. The amount of scatter in the sample paths in either interpretation appears to be comparable for given values of the noise intensity and material statistics. The deterministic solution lies within the scatter in each case.

Figs 5.12(a)-(e) show the effect on the mean and standard deviation of damage and the failure probability (complement of the CDF of $D(t)$ ) using the Stratonovich approach when the conditioning on $D_{0}, A, D_{c}$ and $m$ are removed (in that order). The mean and coefficient of variation (c.o.v.) of the failure time, $T$, are also presented in each figure. A $10 \%$ variability in the initial damage causes no significant increase in the failure probability as a function of time. A $20 \%$ variability in the rate, $A$, causes the variability in $T$ to rise from about $5.3 \%$ to $20.3 \%$. Introducing a $10 \%$ variability in $D_{c}$ has almost no effect on the failure probability at this stage. However, the present model is found to be extremely sensitive to randomness in the exponent, $m$. Introducing a mere $1 \%$ variability to $m$ causes the c.o.v. of the failure time to jump to $43.3 \%$. These values may be compared with the experimental scatter reproduced in Table 4.7 and particularly with the 1749 hour mean rupture time and $21 \%$ c.o.v. observed by Garofalo et al (1961) under the same conditions.

Figs 5.13(a)-(e) show the mean, standard deviation and cumulative failure probability ${ }^{16}$ of creep damage using the Ito interpretation of SDE (5.85). The material properties and the noise intensity are the same as in Figs 5.12. A comparison of the two approaches in the five different scenarios, shows that the former results in a marginal increase in the mean time to failure, $\mu_{T}$, but provides a correspondingly lower estimate of the c.o.v., $V_{T}$. The Stratonovich solution therefore provides a slightly more pessimistic estimate of

\footnotetext{
${ }^{14} D_{0}$ is assumed to be Gaussian when treated as random: its mean is computed with the help of eq (4.33) as in sec. 4.3.2 with deterministic $E, K, \sigma_{f}, M$ (Table 4.4), and its c.o.v. is assumed to be $10 \%$. Computed this way, the mean initial damage in type 316 stainless steel at $1100^{\circ} \mathrm{F}$ under $199 \mathrm{MPa}$ stress is 0.010787 .

${ }^{15}$ When a material parameter is treated as random, its mean is taken equal to its nominal value. The nominal creep law parameters for type 316 stainless steel are listed in Table 4.4. When treated as random variables, $A$ is assumed to be lognormal with c.o.v. $20 \%, m$ and $D_{c}$ are assumed to be normal with c.o.v.'s $1 \%$ and $10 \%$ respectively, and they are considered statistically independent of each other and of $D_{0}$. The mean of $D_{c}$ is assumed to be 0.2 .

${ }^{16} \mu_{D}(t), \sigma_{D}(t)$ and $F_{T}(t)$ are computed numerically with a uniform time-step of $10 \mathrm{hr}$.
} 
reliability than the Ito solution.

The stochastic model of creep damage growth presented in this section is able to account for material variability as well as noise in the process. While it remains to be ascertained whether the Ito or the Stratonovich interpretation provides a closer description of the random creep damage growth phenomenon, the computationally efficient closedform Stratonovich solution (which provides a more conservative estimate of the failure time) may nevertheless be used to find the mean and variance functions of damage, and to estimate the cumulative failure probability in terms of the CDF of the damage function conveniently. The predicted mean damage is found to be close to the deterministic solution from Chapter 4, and experimental results are found to be within the predicted scatter.

\subsection{Stochastic Fatigue Damage}

\subsubsection{Equations and Stochastic Characterization}

As in ductile and creep damage, the mechanistic bases of stochastic fatigue damage accumulation are assumed to remain the same as in its deterministic formulation. The free energy, $\psi$, its partial derivative, $\psi_{D}$, and the surface energy, $\gamma$, may therefore be used directly from sections 4.1 and 4.4 .

Fatigue damage in a given cycle, it may be recalled, is assumed to accumulate only during the loading portions of the stress-strain loops when the stress is greater than the endurance limit. The accumulated damage at the end of any given cycle is therefore the solution of a differential equation describing monotonic damage growth along the reloading curve between the limits mentioned above, with the damage at the end of the previous cycle taken as the initial damage. In the stochastic context, this may be expressed for the $i$ th cycle as:

$$
\frac{d D}{d \epsilon}=\left\{\begin{array}{lll}
-\sigma_{\infty} / \psi_{D}-\left(\sqrt{c_{2}} / c_{1}\right) \xi(\epsilon) / \psi_{D} & ; & \sigma_{\infty} \geq S_{e} \geq 0, \dot{\epsilon}>0 \\
0 & ; & \text { otherwise }
\end{array}\right.
$$

with the initial condition $D=D_{i-1}$. Damage growth can then be represented as the sum of the increments:

$$
\begin{aligned}
D_{i+1} & =D_{i}+\Delta D_{i}, \quad \Delta D_{i} \geq 0, \quad i=1, \ldots, N_{f}-1 \\
D_{N_{f}-1} & <D_{c} \\
D_{N_{f}} & \geq D_{c}
\end{aligned}
$$

where $D_{c}$ is the critical damage and $N_{f}$ is the number of cycles to failure. In the stochastic context, $D_{c}$ and $N_{f}$ are random variables. 
If $\left(\epsilon_{\min }, \sigma_{\min }\right)$ denotes the position of the lower hysteresis loop tip, then a new set of coordinates, $\Delta \epsilon-\Delta \sigma$, denoting the strain and stress ranges can be defined with its origin at the lower loop tip:

$$
\begin{aligned}
\Delta \epsilon & =\epsilon-\epsilon_{\min } \\
\Delta \sigma & =\sigma-\sigma_{\min }
\end{aligned}
$$

such that

$$
d \epsilon=d \Delta \epsilon
$$

and the white noise $\xi(\Delta \epsilon)$, shifted on the strain axis, retains all its characteristics. The stochastic differential equation describing damage growth in cycle $i$ is then:

$$
d D(\Delta \epsilon)=\frac{[1-D(\Delta \epsilon)]\left[\left(\Delta \epsilon_{p}\right)^{1 / M^{\prime}}-\left(\Delta \epsilon_{p 1_{i}}\right)^{1 / M^{\prime}}\right] d \Delta \epsilon_{p}+\left(\sqrt{c_{2}} / c_{1}\right) d W(\Delta \epsilon)}{\frac{1}{1+\frac{1}{M^{\prime}}}\left[\Delta \epsilon_{p}^{1+1 / M^{\prime}}-\Delta \epsilon_{p 0_{i}}^{1+1 / M^{\prime}}\right]-\Delta \epsilon_{p 1_{i}}^{1 / M^{\prime}}\left(\Delta \epsilon_{p}-\Delta \epsilon_{p 0_{i}}\right)+\frac{3}{4} \sigma_{f} / K_{1}}
$$

where $\Delta \epsilon_{p}$ is the plastic strain range, $\Delta \epsilon_{p 1_{i}}$ is the plastic strain range corresponding; to $\epsilon=0, \Delta \epsilon_{p 0_{i}}$ is the plastic strain range corresponding to $\sigma=S_{e}, \quad \sigma_{f}$ is the true failure stress, $M^{\prime}$ is the cyclic hardening exponent, $K_{1}=2^{1-1 / M^{\prime}} H$ where $H$ is the cyclic hardening modulus and the ratio $K^{\prime} / E$ has been neglected. Detailed descriptions of these parameters are given in sec 4.4. $d W(\Delta \epsilon)$ denotes the standard Wiener process, indexed by the strain range. The damage, $D_{i}$, at the end of cycle $i$ is the solution of eq (5.109) at $\Delta \epsilon_{p}=\Delta \epsilon_{p m_{i}}$ with the initial condition $D=D_{i-1}$ at $\Delta \epsilon_{p}=\Delta \epsilon_{p 0_{i}}$.

Introducing $X=1-D$, eq (5.109) can be rewritten as

$$
d X=-a X d \Delta \epsilon_{p}-b d W\left(\Delta \epsilon_{p}\right)
$$

where

$$
\begin{aligned}
a\left(\Delta \epsilon_{p}\right) & =\frac{\Delta \epsilon_{p}^{1 / M^{\prime}}-\Delta \epsilon_{p 1_{i}}^{1 / M^{\prime}}}{\frac{\Delta \epsilon_{p}^{1+1 / M^{\prime}}}{1+\frac{1}{M}}-\Delta \epsilon_{p} \Delta \epsilon_{p 1_{i}}^{1 / M^{\prime}}+C_{i}} \\
b\left(\Delta \epsilon_{p}\right) & =\frac{C_{0}}{\frac{\Delta \epsilon_{p}^{1+1 / M^{\prime}}}{1+\frac{1}{M}}-\Delta \epsilon_{p} \Delta \epsilon_{p 1_{i}}^{1 / M^{\prime}}+C_{i}} \\
C_{0} & =\frac{\sqrt{c_{2}}}{c_{1} K_{1}} \\
C_{i} & =\frac{3}{4} \frac{\sigma_{f}}{K_{1}}-\frac{1}{1+\frac{1}{M^{\prime}}} \Delta \epsilon_{p 0_{i}}^{1+1 / M^{\prime}}+\Delta \epsilon_{p 1_{i}}^{1 / M^{\prime}} \Delta \epsilon_{p 0_{i}}
\end{aligned}
$$

The drift term, $b$, in eq (5.110) is independent of damage, $D$. Consequently, the Ito and Stratonovich solutions of eq (5.110) are identical. The solution, moreover, can be 
obtained in closed-form by adopting an approach similar to that used for eq (5.60) in stochastic ductile damage:

$$
X\left(\Delta \epsilon_{p}\right)=X\left(\Delta \epsilon_{p 0_{i}}\right) \frac{\frac{\Delta \epsilon_{p 0_{i}}^{1+1 / M^{\prime}}}{1+\frac{1}{M}}-\Delta \epsilon_{p 0_{i}} \Delta \epsilon_{p 1_{i}}^{1 / M^{\prime}}+C_{i}}{\frac{\Delta \epsilon_{p}^{1+1 / M^{\prime}}}{1+\frac{1}{M}}-\Delta \epsilon_{p} \Delta \epsilon_{p 1_{i}}^{1 / M^{\prime}}+C_{i}}+\frac{C_{0}\left[W\left(\Delta \epsilon_{p}\right)-W\left(\Delta \epsilon_{p 0_{i}}\right)\right]}{\frac{\Delta \epsilon_{p}^{1+1 / M^{\prime}}}{1+\frac{1}{M}}-\Delta \epsilon_{p} \Delta \epsilon_{p 1_{i}}^{1 / M^{\prime}}+C_{i}}
$$

which gives the damage at the end of cycle $i$ as

$$
D_{i}=1-\frac{\left.\left(1-D_{i-1}\right)(3 / 4)\left(\sigma_{f}\right) / K_{1}\right)}{\frac{\Delta \epsilon_{p m_{i}}^{1+1 / M^{\prime}}}{1+\frac{1}{M}}-\Delta \epsilon_{p m_{i}} \Delta \epsilon_{p 1_{i}}^{1 / M^{\prime}}+C_{i}}+\frac{C_{0} \Delta W_{i}}{\frac{\Delta \epsilon_{p m_{i}}^{1+1 / M^{\prime}}}{1+\frac{1}{M}}-\Delta \epsilon_{p m_{i}} \Delta \epsilon_{p 1_{i}}^{1 / M^{\prime}}+C_{i}}
$$

where $\Delta W_{i}$ is the increment of the Wiener process over the $i$ th cycle. The recursive nature of $\left(1-D_{i}\right)$ in the above equation makes it possible to express damage, $D_{n}$, at the end of $n$ cycles in terms of the initial damage, $D_{0}$, and $n$ independent increments of the Wiener process:

$$
D_{n}=1-\left(1-D_{0}\right)\left(\frac{3 \sigma_{f}}{4 K_{1}}\right)^{n} \prod_{i=1}^{n} g_{i}+C_{0} \sum_{i=1}^{n} \Delta W_{i} \prod_{j=i}^{n}\left(\frac{3 \sigma_{f}}{4 K_{1}}\right) g_{j}
$$

where

$$
g_{i}=\frac{1}{\frac{\Delta \epsilon_{p m_{i}}^{1+1 / M^{\prime}}}{1+\frac{1}{M}}-\Delta \epsilon_{p m_{i}} \Delta \epsilon_{p 1_{i}}^{1 / M^{\prime}}+C_{i}}
$$

If the material parameters, $\underline{\Omega}=\left\{K^{\prime}, M^{\prime}, \sigma_{f}, S_{e}\right\}$, are held constant, the mean of this process is

$$
\mu\left(D_{n} \mid \underline{\Omega}\right)=1-\left(1-\mu\left(D_{0}\right)\right)\left(\frac{3 \sigma_{f}}{4 K_{1}}\right)^{n} \prod_{i=1}^{n} g_{i}
$$

which is identical to the deterministic solution (eq 4.87) of fatigue damage. The variance is

$$
\sigma^{2}\left(D_{n} \mid \underline{\Omega}\right)=\sigma^{2}\left(D_{0}\right)\left(\frac{3 \sigma_{f}}{4 K_{1}}\right)^{2 n}\left(\prod_{i=1}^{n} g_{i}\right)^{2}+C_{0}^{2} \sum_{i=1}^{n}\left[\prod_{j=i}^{n}\left(\frac{3 \sigma_{f}}{4 K_{1}}\right) g_{j}\right]^{2}\left(\Delta \epsilon_{p m_{i}}-\Delta \epsilon_{p 0_{i}}\right)
$$

where $\mu\left(D_{0}\right)$ and $\sigma^{2}\left(D_{0}\right)$ are respectively the mean and variance of the initial damage. If, in addition to holding the material parameters constant, the initial damage is either deterministic or Gaussian, then $D_{n}$ is Gaussian on account of the additive Wiener increments in eq (5.117).

If the fatigue loading is strain-controlled, the function $g_{i}=g$ in eq $(5.118)$ is independent of $i$ and the mean and the variance of damage accumulated after $n$ cycles simplify to:

$$
\mu\left(D_{n} \mid \underline{\Omega}\right)=1-\left(1-\mu\left(D_{0}\right)\right)\left(\frac{3 \sigma_{f}}{4 K_{1}} g\right)^{n}
$$


and

$$
\begin{aligned}
\sigma^{2}\left(D_{n} \mid \underline{\Omega}\right) & =\sigma^{2}\left(D_{0}\right)\left(\frac{3 \sigma_{f}}{4 K_{1}} g\right)^{2 n}+C_{0}^{2}\left(\Delta \epsilon_{p m}-\Delta \epsilon_{p 0}\right) \sum_{i=1}^{n}\left[\frac{3 \sigma_{f}}{4 K_{1}} g\right]^{2(n-i+1)} \\
& =\sigma^{2}\left(D_{0}\right)\left(\frac{3 \sigma_{f}}{4 K_{1}} g\right)^{2 n}+C_{0}^{2}\left(\Delta \epsilon_{p m}-\Delta \epsilon_{p 0}\right)\left[\frac{3 \sigma_{f}}{4 K_{1}} g\right]^{2} \frac{\left[\frac{3 \sigma_{f}}{4 K_{1}} g\right]^{2 n}-1}{\left[\frac{3 \sigma_{f}}{4 K_{1}} g\right]^{2}-1}
\end{aligned}
$$

Also, under strain-controlled fatigue, the conditional cumulative distribution function. (CDF) of damage at the end of $n$ cycles is:

$$
\mathrm{P}\left[D_{n} \leq d \mid \underline{\Omega}, D_{0}\right]=\Phi\left[\frac{\left(1-D_{0}\right)\left[\frac{3 \sigma_{f}}{4 K_{1}} g\right]^{n}-(1-d)}{C_{0}\left(\Delta \epsilon_{p m}-\Delta \epsilon_{p 0}\right)\left[\frac{3 \sigma_{f}}{4 K_{1}} g\right]^{2}\left(\left[\frac{3 \sigma_{f}}{4 K_{1}} g\right]^{2 n}-1\right) /\left(\left[\frac{3 \sigma_{f}}{4 K_{1}} g\right]^{2}-1\right)}\right]
$$

since the Wiener increments in any two different cycles are statistically independent. If the material properties and initial damage are random and if their joint probability density function is known, the unconditional mean, variance and CDF of damage may be obtained with the help of the theorem of total probability.

It may be more convenient in some situations to describe damage and its statistics as a function of time, instead of the number of load cycles. If the cycling rate is known, then $D(t), \mu_{D}(t)$ etc may be obtained by simply substituting the functional relation, $n(t)$, in the above formulation. If however, the number of cycles, $N(t)$, is random, then $D(t)$ and its statistics must be obtained using the stochastic description of $N(t)$.

Assuming that the damage growth process described by eq (5.117) is almost always positive, the cumulative probability of failure is the complement of the CDF of $D_{n}$ :

$$
\mathrm{P}\left[N_{I} \leq n\right]=F_{N_{I}}(n) \simeq \mathrm{P}\left[D_{n}>D_{c}\right]
$$

from which the probability distribution of $N_{I}$ may be obtained if the statistics of $D_{n}$ and $D_{c}$ are known.

\subsubsection{Validation and Studies in Reliability}

A CDM-based model of fatigue damage and failure concerns the fatigue initiation period only. Consequently, the predictions and validation of the above formulation should be restricted to the random characteristics of only the initiation life, and should not be extrapolated to the crack propagation phase leading to fracture.

The fatigue crack initiation time, $N_{I}$, and propagation time, $N_{P}$, are both random, and the statistics of their sum, $N_{T}=N_{I}+N_{P}$, depends in general on their correlation structure. However, the probabilistic estimation of the total life, $N_{T}$, can be simplified through the assumption that the initiation and propagation times are independent of one 
another. The variability in $N_{I}$ is due to the fluctuations in energy and material properties throughout the material volume (a necessary assumption of CDM). The variability in $N_{p}$ on the other hand depends solely on the local fluctuations around the crack-tip (which is the basic tenet of fracture mechanics). Similar arguments were put forward by Min et al (1996): "the physical mechanism of fatigue damage accumulation in the crack initiation process is very different from that of in [sic] the crack growth process".

As in the case of random creep damage, no published data on random fatigue damage growth oriented toward CDM could be located. There are limited data available on the random initiation life, but these data do not provide information on CDM-based damage growth during the pre-initiation stage.

In the following, studies of random fatigue damage growth in A106-B (a carbon steel) at $288^{\circ} \mathrm{C}$ in air, the material properties are taken from table 4.8 and are treated deterministically. The noise parameter, $\sqrt{c_{2}} / c_{1}$ is derived from Keisler et al (1994). By fitting a least-squares curve between the predicted and observed number of cycles to failure, Keisler et al (1994) obtained the variance of the residue, $z=\ln \left(N_{\text {pred }}\right)-\ln \left(N_{o b s}\right)$, as 0.276 for tests conducted in air on the same material. Assuming $z$ to be Gaussian, the coefficient of variation (COV) of $\exp (z)$ is 0.56 . The least-squares curve was found to produce a good fit and the above residue is assumed to occur solely due to the noise in the process. Considering $D_{c}=0.25$ (deterministic) and $D_{0}=0$ (deterministic), eq. (5.124) was solved repeatedly and the COV of $N_{I}$ for several strain amplitudes $(0.002$ through 0.006$)$ and noise parameters $\sqrt{ } c_{2} / c_{1}(200-1000 \mathrm{MPa})$ were obtained. Assuming that $N_{I}$ (in the absence of material variability) and $\exp (z)$ have the same $\operatorname{COV}, \sqrt{c_{2}} / c_{1}$ of approximately $1000 \mathrm{MPa}$ produced COV's for $N_{I}$ in the range $0.4 \sim 0.6$. Consequently, $\sqrt{c_{2}} / c_{1}=1000 \mathrm{MPa}$ is used in the examples presented here.

Fig 5.14 predicts random fatigue damage growth in A106-B carbon steel due to fullyreversed strain-controlled cycling $\left(\epsilon_{a}=0.003\right)$ at $288^{\circ} \mathrm{C}$ in air. The mean damage, $\mu_{D}(n)$, and the range of one standard deviation above and below the mean, $\mu_{D}(n) \pm \sigma_{D}(n)$, are plotted in the figure.

Fig 5.15 shows the predicted mean time to crack initiation, $\mu_{N_{I}}$, (obtained using eq 5.124 with $\sqrt{c}_{2} / c_{1}=1000 \mathrm{MPa}, D_{\mathrm{c}}=0.25$ and $\left.D_{0}=0\right)$ and compares the predictions with the observed cycles to failure, $N_{T}$, (Chopra, 1996) ${ }^{17}$ for the same material and conditions as described above. Considering $N_{I}$ and $N_{P}$ to be statistically independent, as discussed at the beginning of this subsection, the variance of $N_{T}$ should be significantly higher than that of $N_{I}$; and a plot of one standard deviation (predicted) above and below the predicted mean $N_{I}$ is found to be tighter than the experimental scatter in $N_{T}$. The prediction also agrees with the generally observed trend in fatigue cycling that at lowcycle fatigue, $N_{P}$ takes up most of the total life, whereas in high-cycle fatigue, the crack initiation phase consumes most of $N_{T}$.

\footnotetext{
${ }^{17}$ Failure is defined by Chopra and his colleagues (e.g., Keisler et al, 1994) as a $25 \%$ drop in the peak stress, which corresponds to approximately a $3 \mathrm{~mm}$ crack, and is quite close to the point of rupture.
} 
Fig 5.16 plots the predicted number of cycles for four different probabilities of crack: initiation: $0.01,0.05,0.25$ and 0.50 , over a range of strain amplitudes for the same material and test conditions, and compared them with the experimental number of cycles to failure (Chopra, 1996). As before, $D_{c}=0.25$ (deterministic) and $D_{0}=0$ (deterministic). The predicted probability of crack initiation agrees qualitatively with that by Keisler et al (1994).

The randomness in fatigue life is a function of the initiation as well as the propagation lives, and a consideration of both is needed for a complete understanding of the phenomenon. Available models of random fatigue crack growth (discussed in section 2.3.2) ignore the fundamental problem of when the crack may initiate in a smooth. specimen. The stochastic CDM approach shows promise for reducing the empiricism of current approaches to predicting crack initiation.

\subsection{Closure}

Stochastic differential equations of damage growth for ductile deformation, creep and fatigue are obtained starting from the first principles of thermodynamics, and recognizing the intrinsic energy fluctuations in matter. Closed-form solutions in each case are also obtained: however, while a closed-form Stratonovich solution is possible in all three cases, closed-form Ito solutions are available only for ductile deformation and fatigue.

The present approach is well-suited to model noise in the damage growth process, and it can tackle variabilities in material parameters, as well as in the initial and critical damages. Simple reliability computations were also performed which showed that the probability distribution function of $D(t)$ can substitute for the more difficult to compute reliability function $L(t)$, as damage growth is almost always positive. In the following chapter, more detailed cases of damage growth, possible synergistic effects and the role of corrosion in reliability will be investigated. 


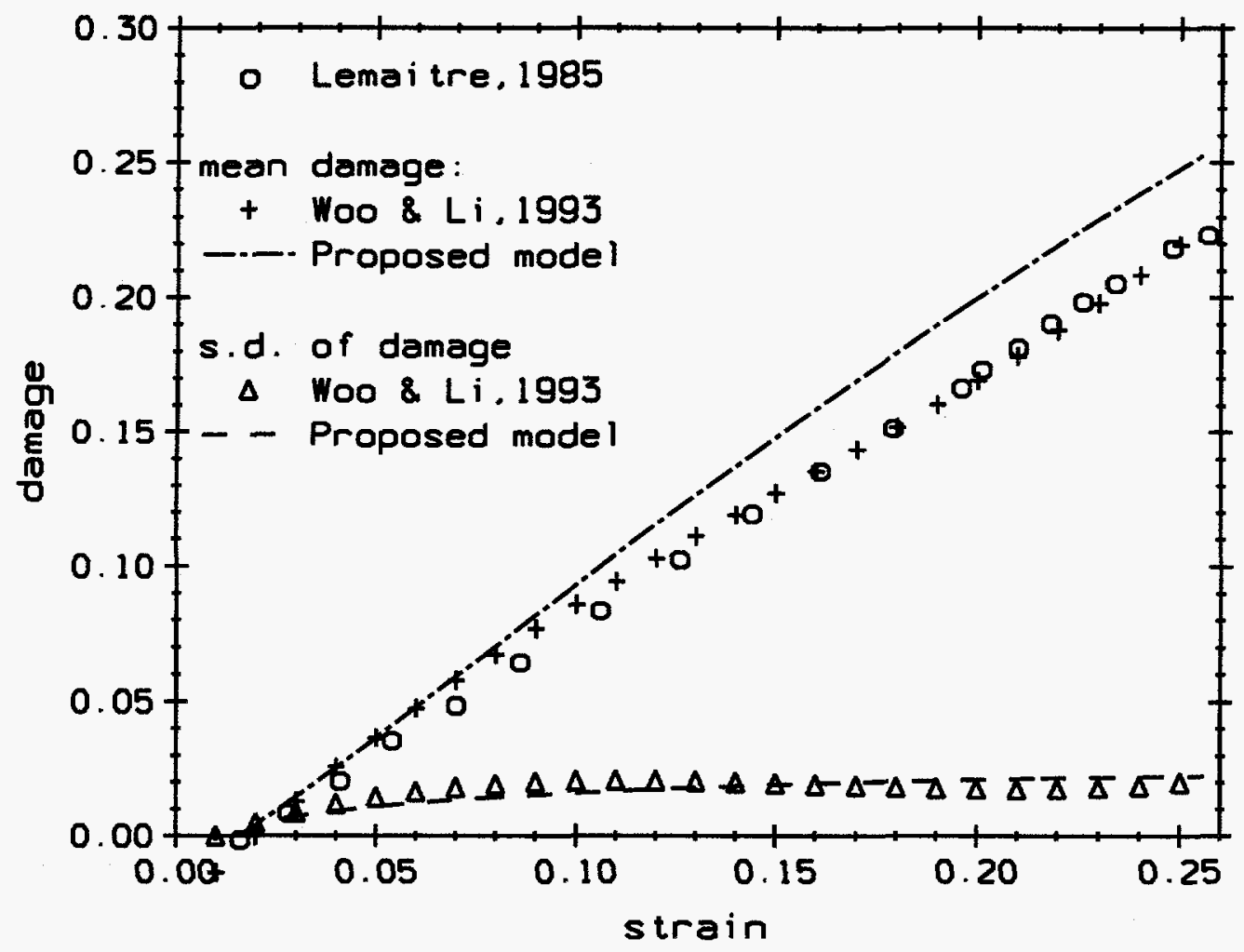

Figure 5.1: Stochastic ductile damage growth in 2024-T3 Aluminum under deterministic initial condition $\left(\epsilon_{0}, D_{0}\right)$ and material parameters $\left(\sigma_{f}, K, M\right)$. 

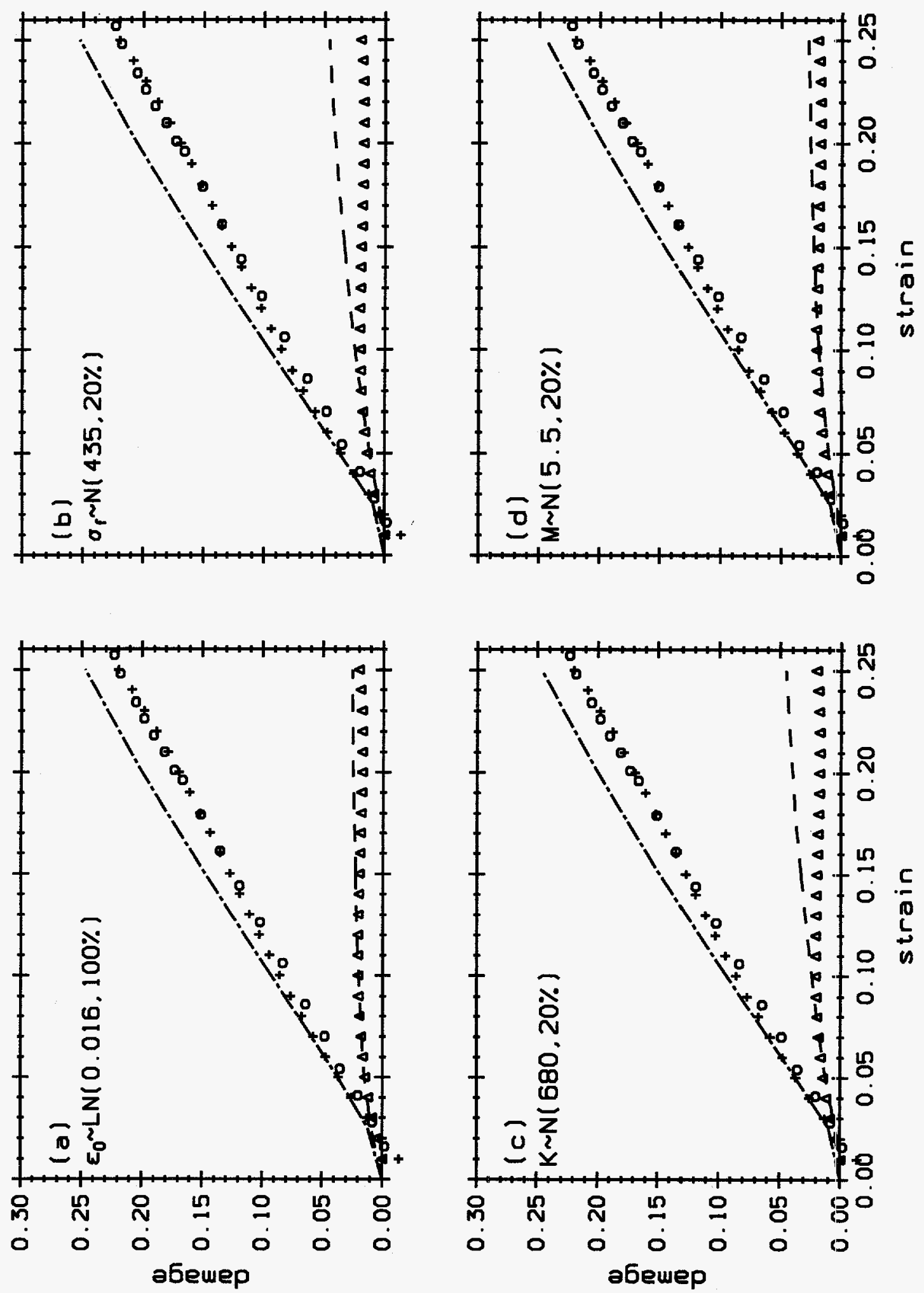

Figure 5.2: Effect of randomness in material parameters and initial damage on stochastic ductile damage growth in 2024-T3 Aluminum (one variable treated as random at a time) 

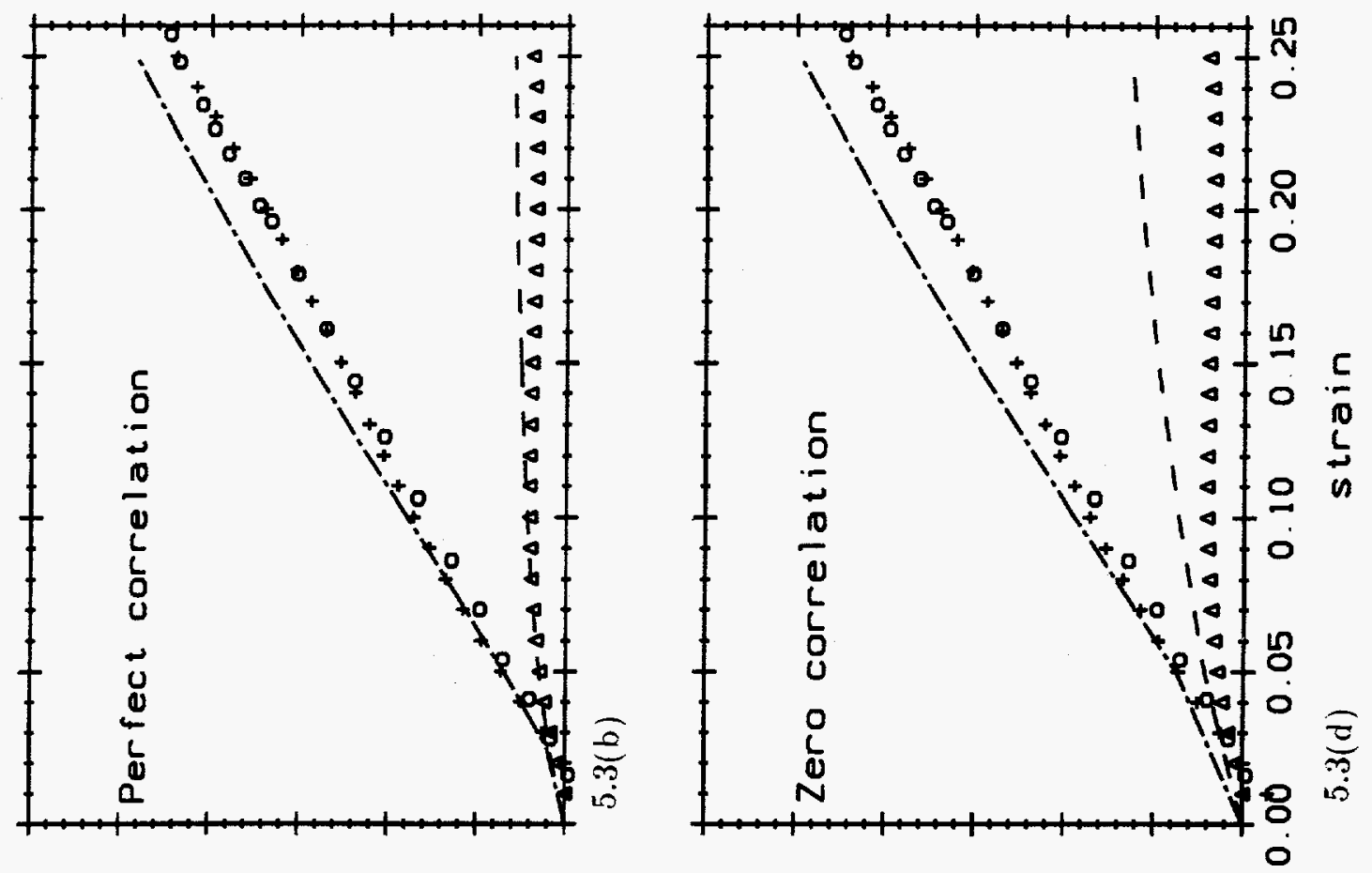

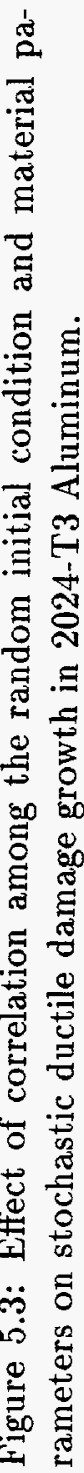
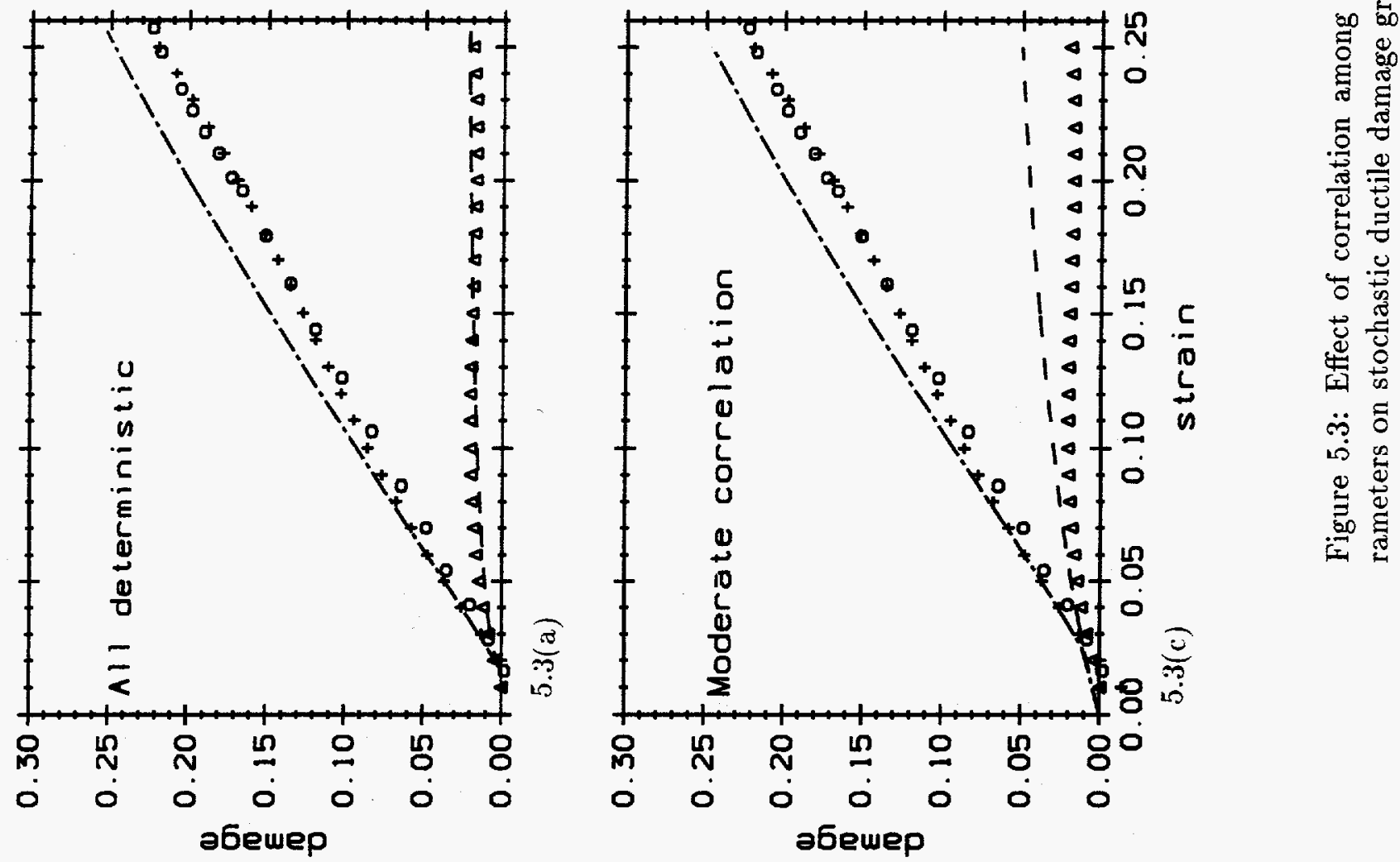

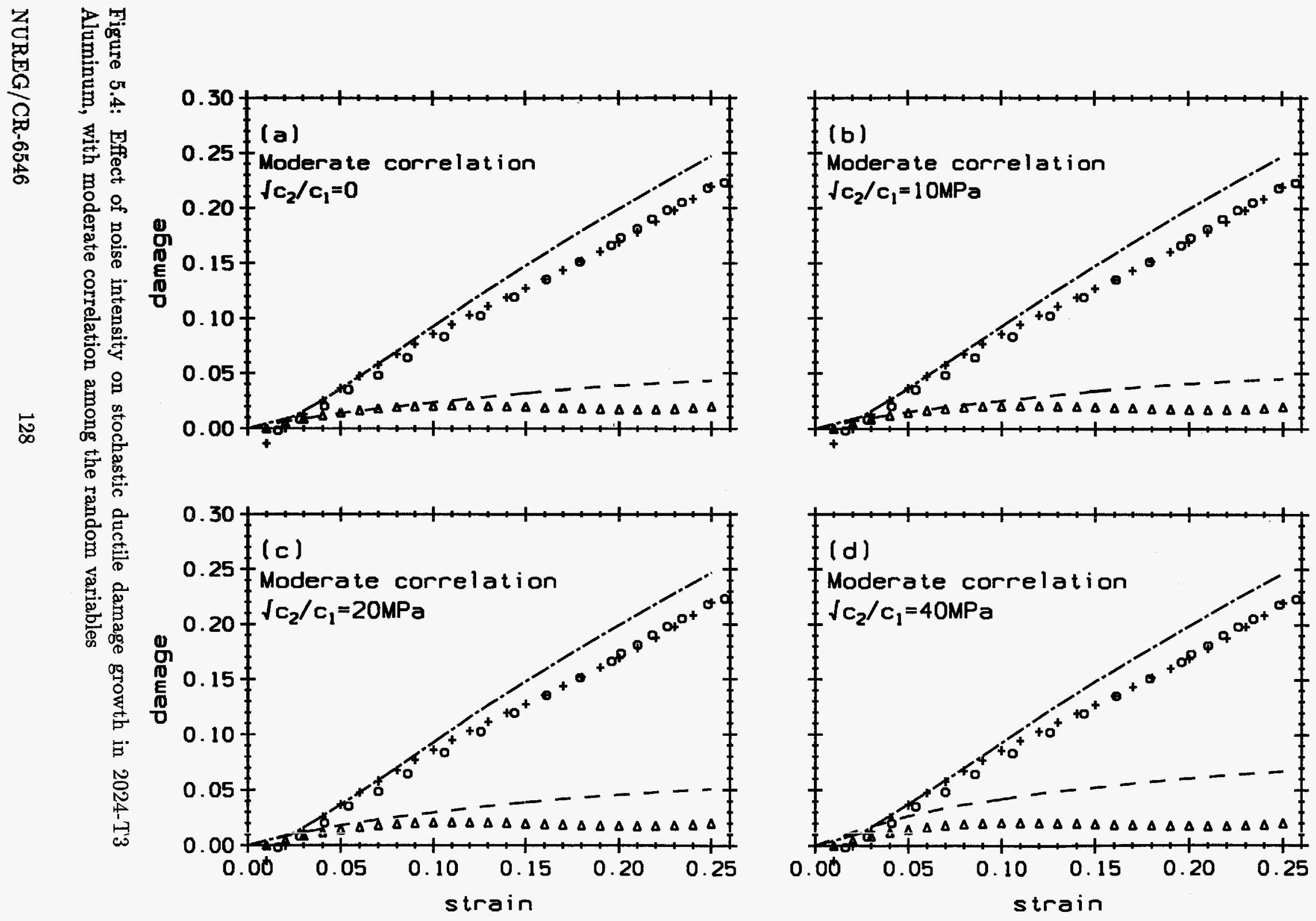

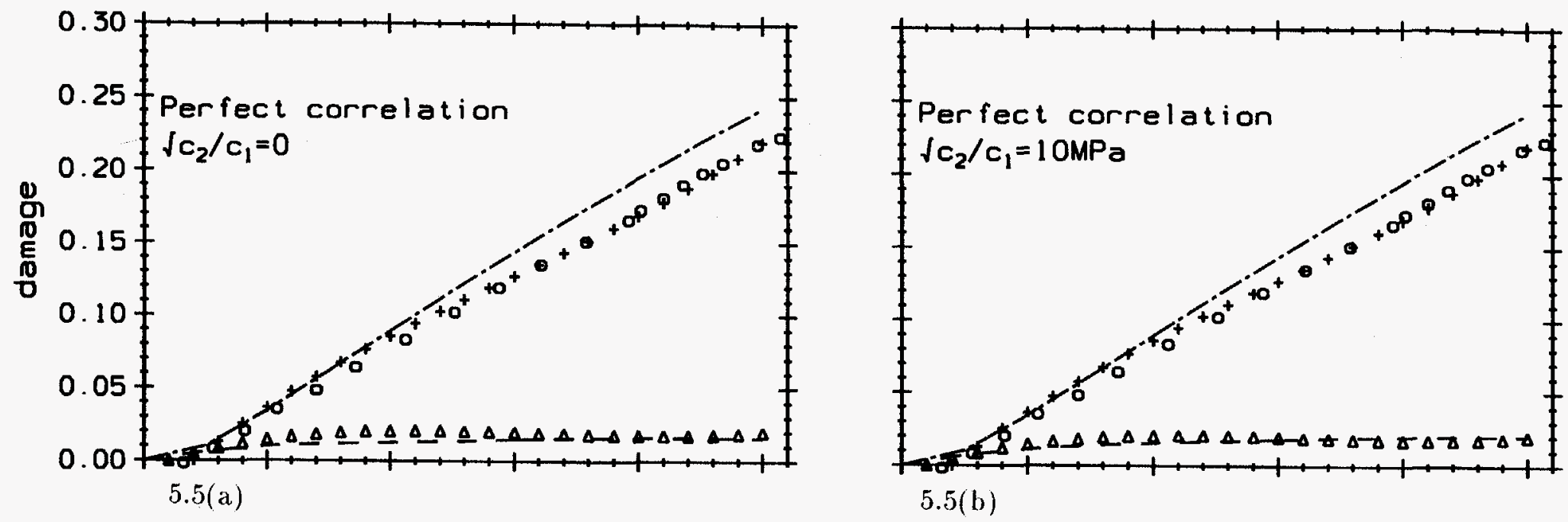

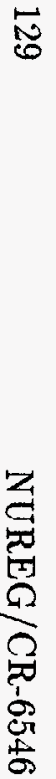
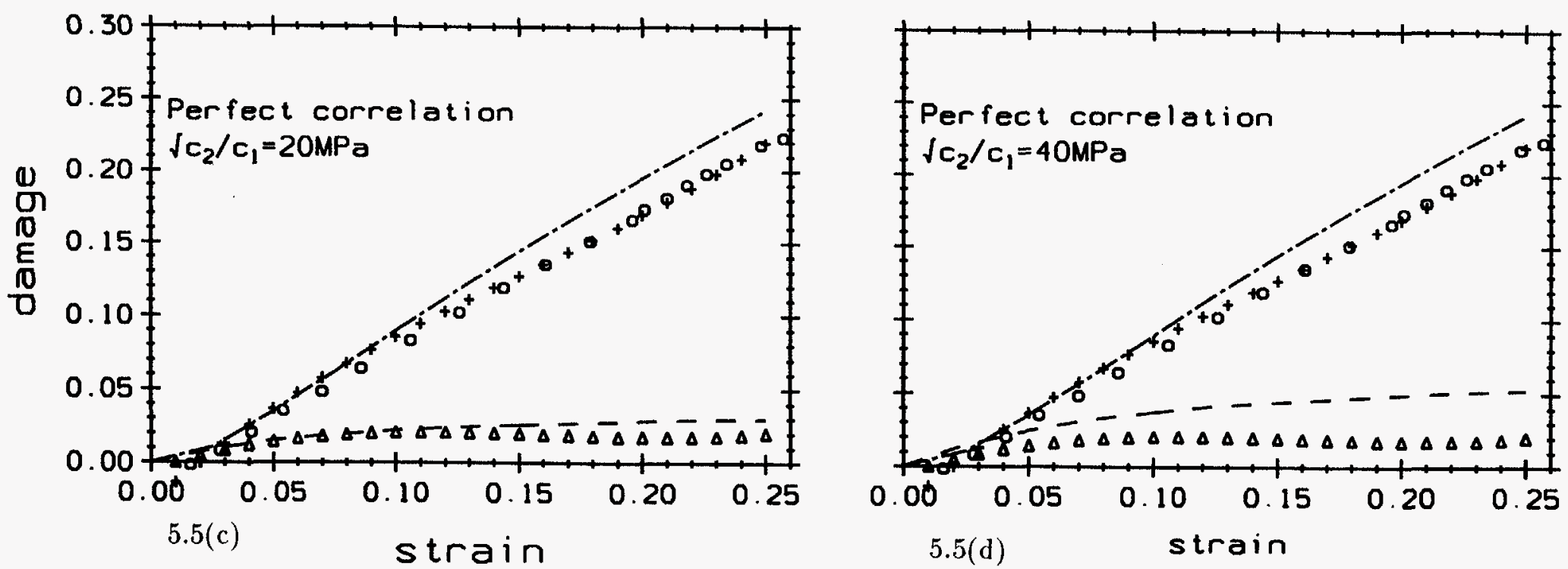

Figure 5.5: Effect of noise intensity on stochastic ductile damage growth in 2024-T3 Aluminum, with perfect correlation among the random variables. 


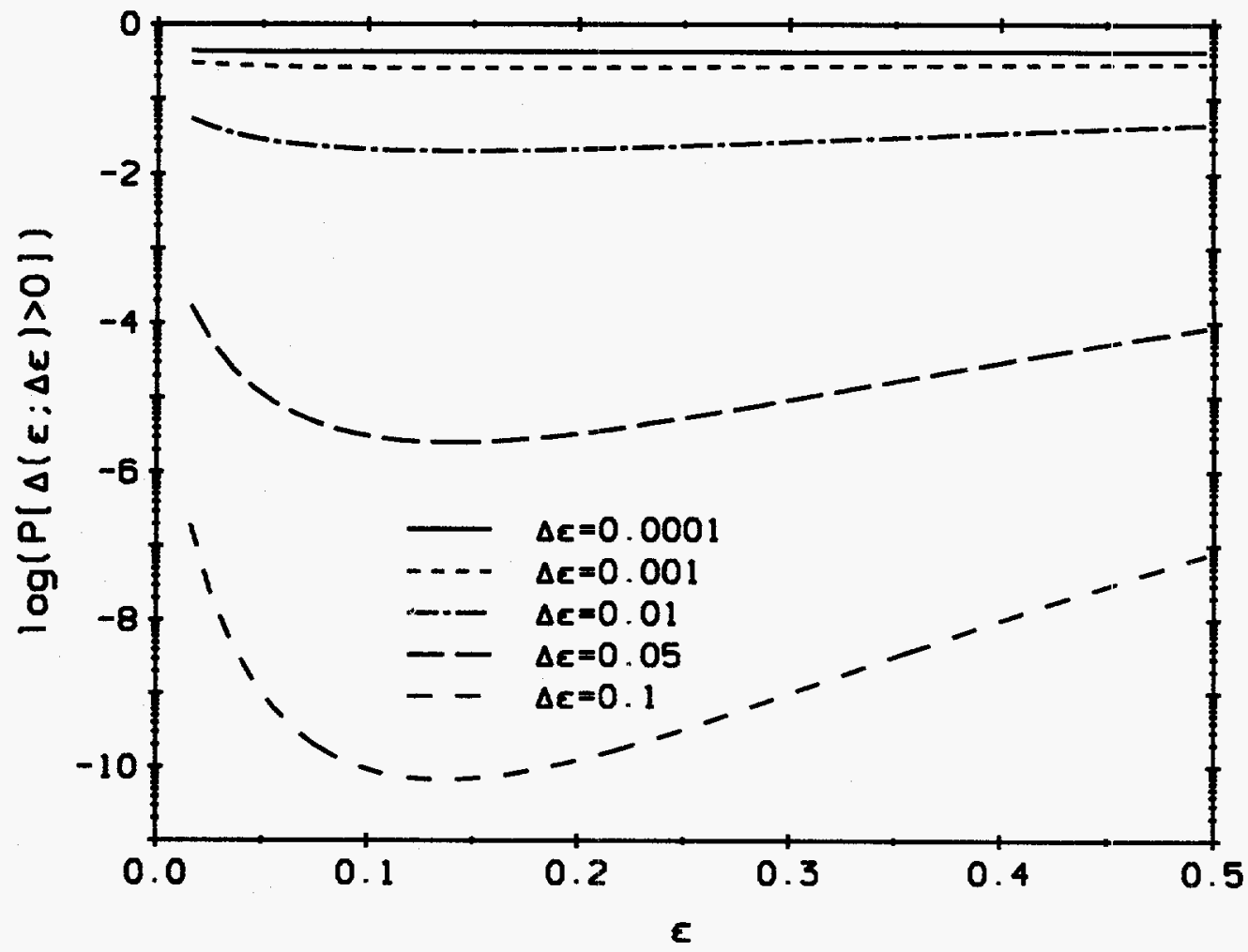

Figure 5.6: Probability of negative damage increment in the proposed model 

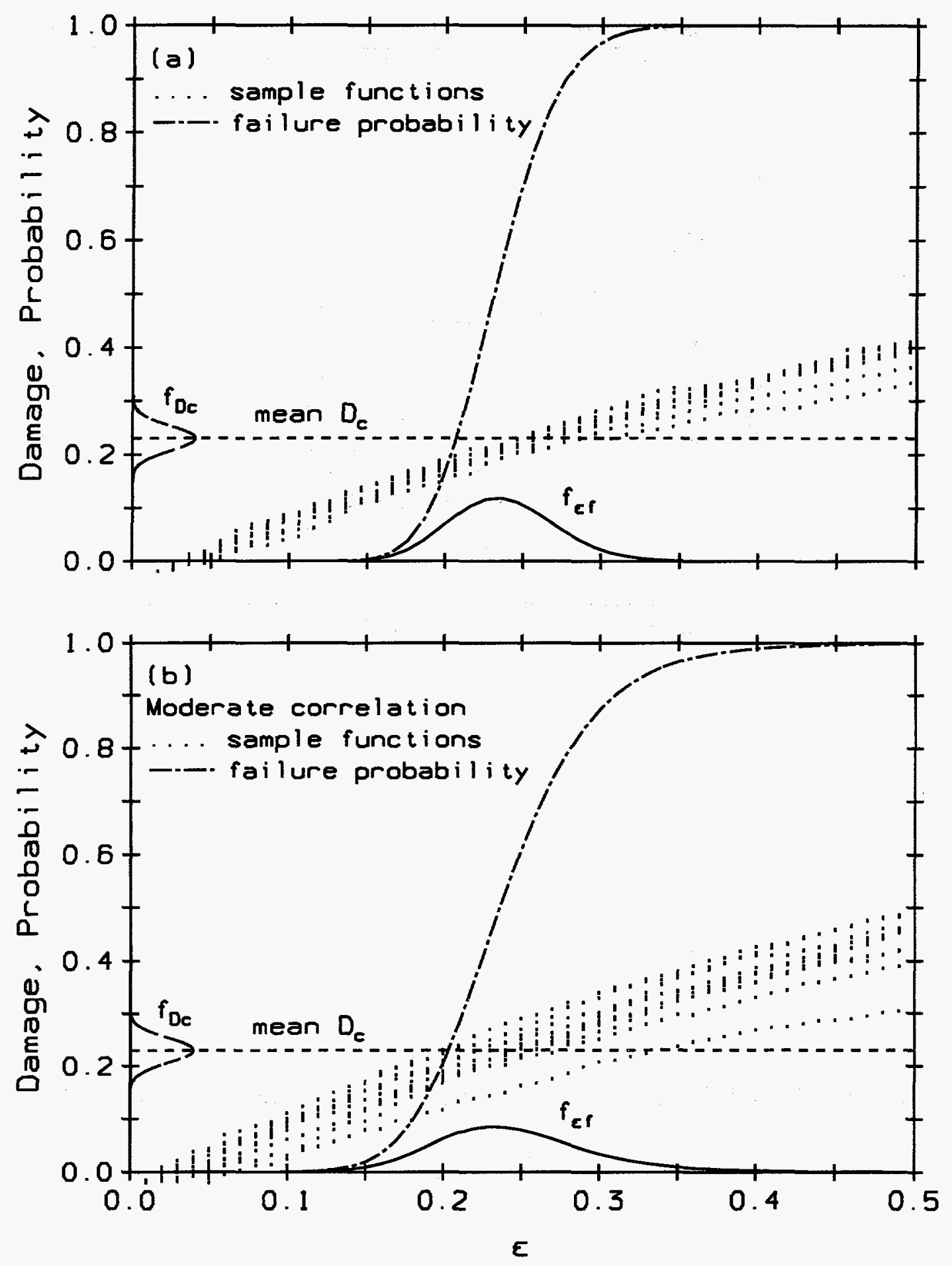

Figure 5.7: Failure probability, sample paths of damage and probability distribution of $D_{c}$ and $\epsilon_{f}$ in 2024-T3 Aluminum (a)only $D_{c}$ is random (b) all five parameters random. 

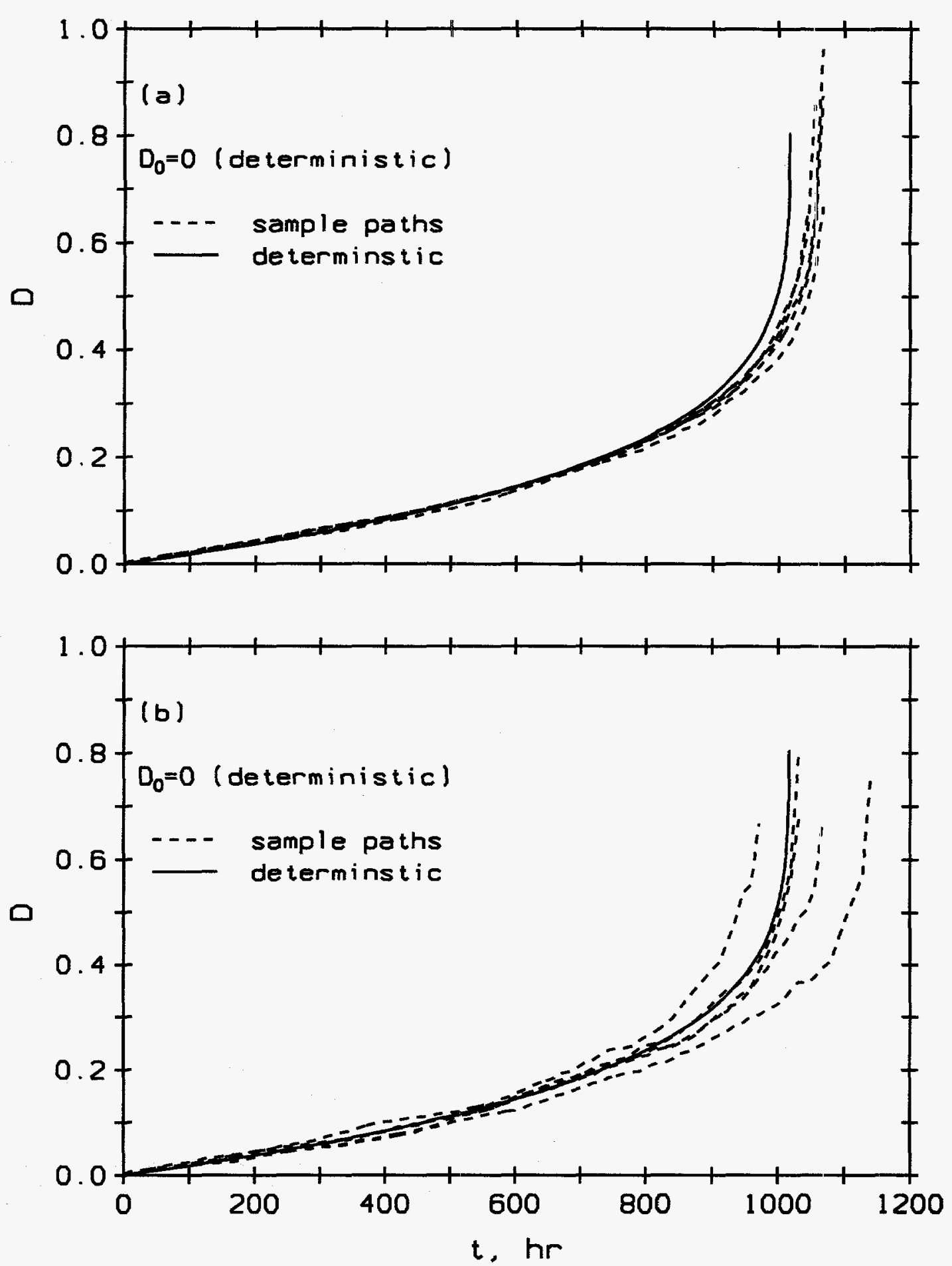

Figure 5.8: Dependence of creep damage on noise intensity in A36 steel stressed to $12 \mathrm{ksi}$ at $1000^{\circ} \mathrm{F}$ (material parameters deterministic), (a) $\sqrt{c_{4}} / c_{3}=10 \mathrm{ksi} \sqrt{\mathrm{hr}}$ and (b) $\sqrt{c_{4}} / c_{3}=$ $20 k s i \sqrt{h r}$ 

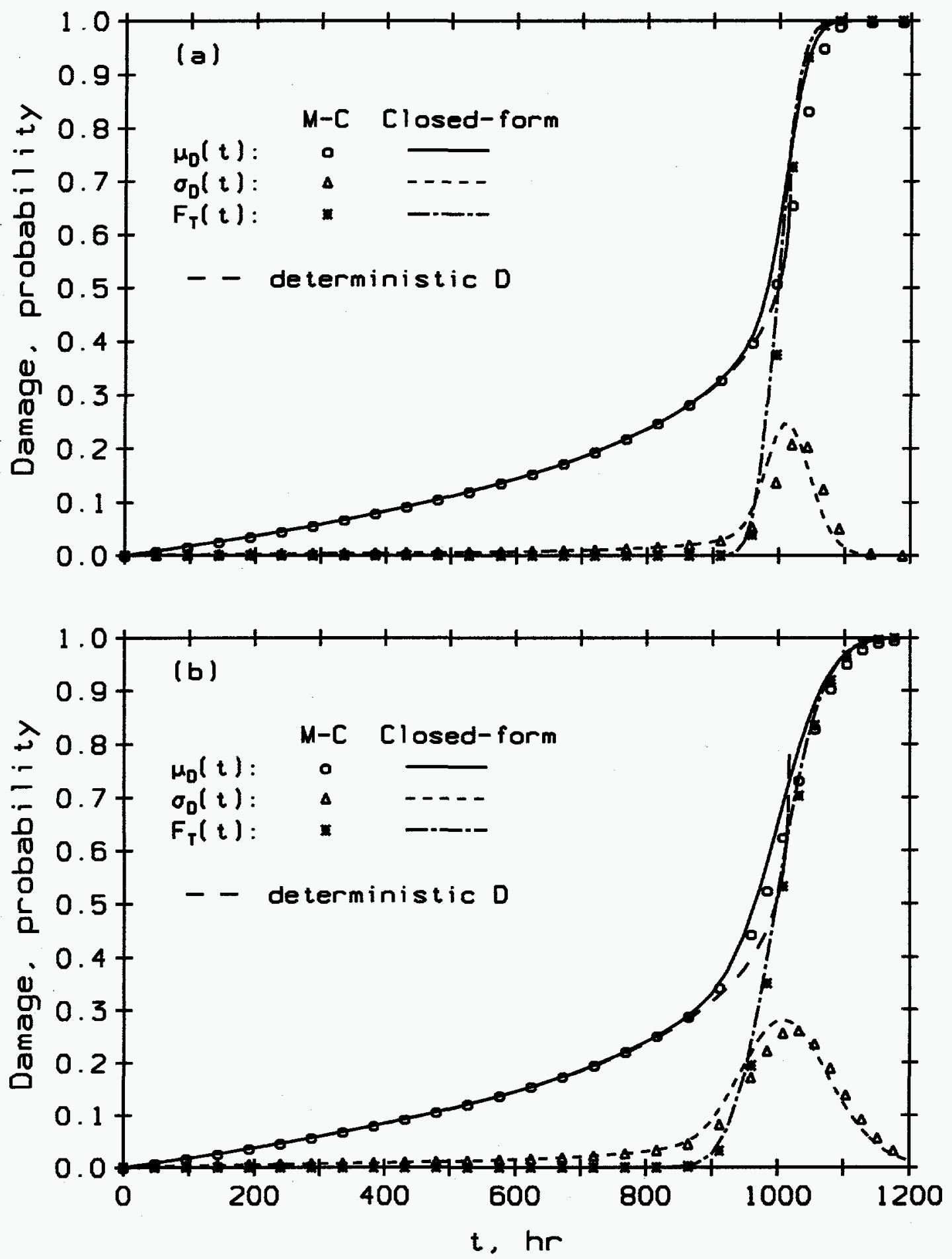

Figure 5.9: Statistics of damage, failure probabilities in creep of A36 steel stressed to $12 \mathrm{ksi}$ at $1000^{\circ} \mathrm{F}$, (a) $\sqrt{c_{4}} / c_{3}=10 \mathrm{ksi} \sqrt{h r}$ and (b) $\sqrt{c_{4}} / c_{3}=20 \mathrm{ksi} \sqrt{h r}$ 

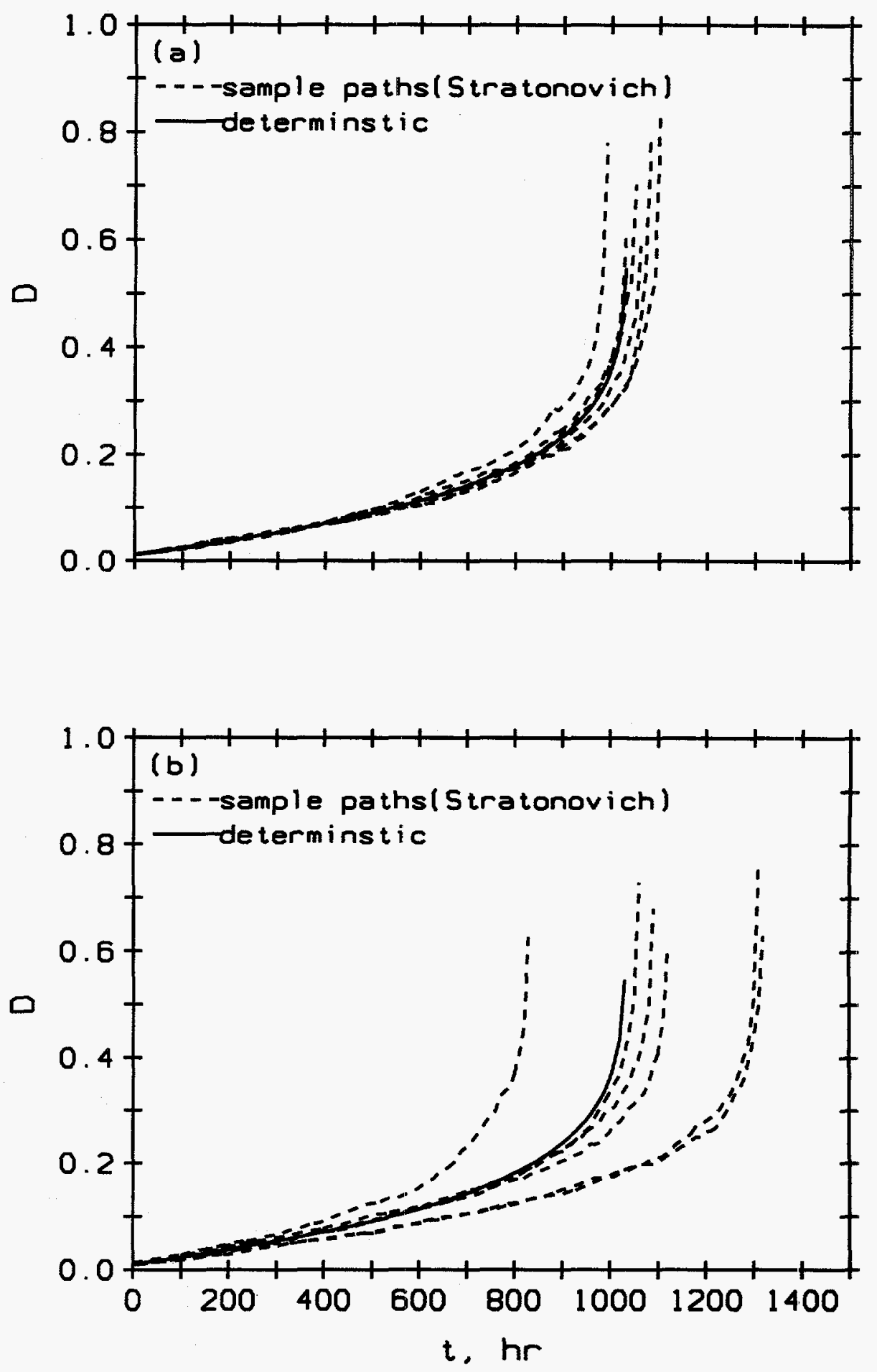

Figure 5.10: Sample paths of creep damage (under Stratonovich interpretation) in type 316 stainless steel stressed to $199 \mathrm{MPa}$ at $1100^{\circ} \mathrm{F},\left(\sqrt{c_{4}} / c_{3}=300 \mathrm{MPa} \sqrt{h r}\right)\left(\right.$ a) $A, m, D_{0}$ deterministic, and (b) $A, m, D_{0}$ random. 

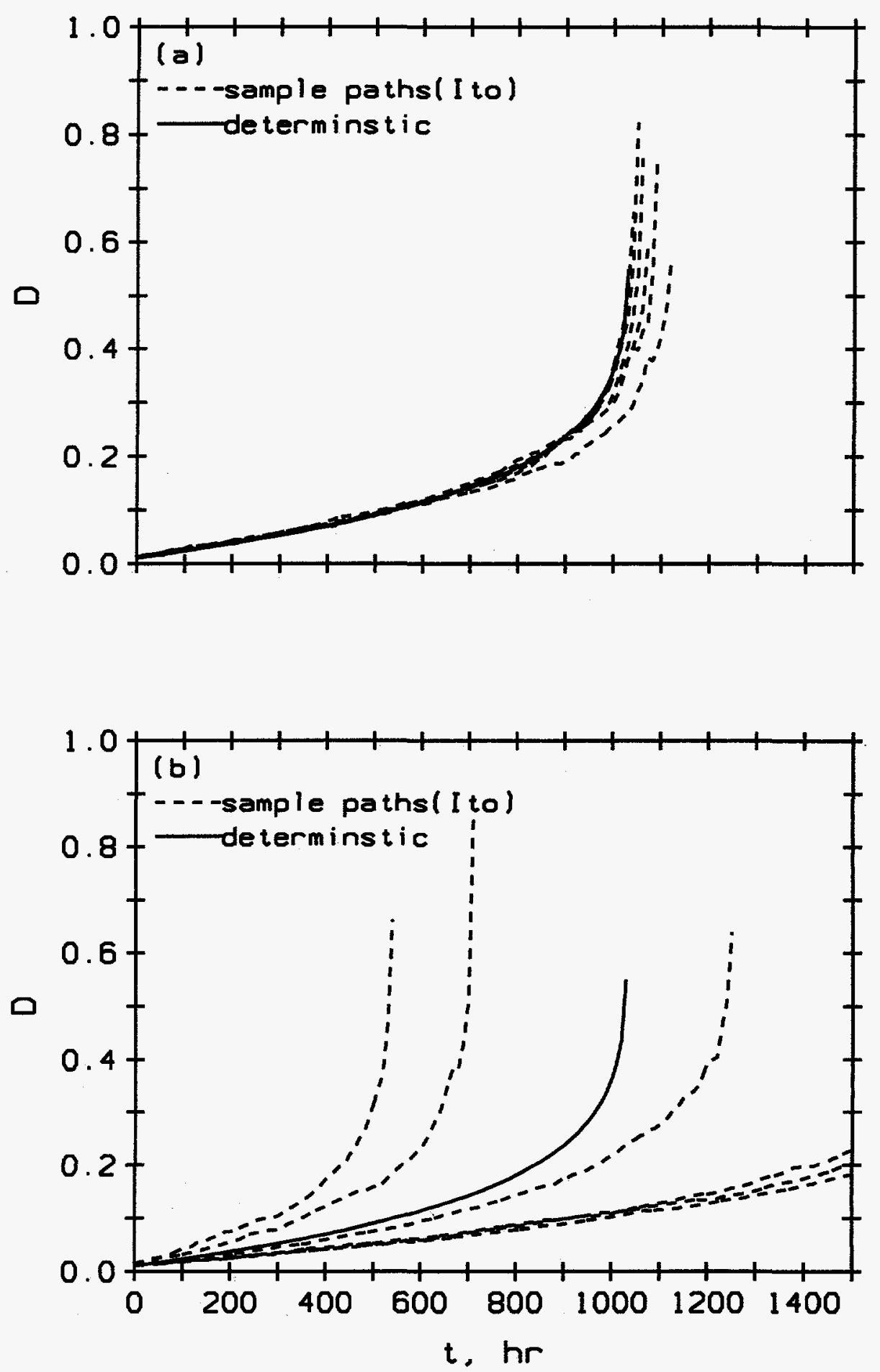

Figure 5.11: Sample paths of creep damage (under Ito interpretation) in type 316 stainless steel stressed to $199 \mathrm{MPa}$ at $1100^{\circ} \mathrm{F},\left(\sqrt{c_{4}} / c_{3}=300 \mathrm{MPa} \sqrt{h r}\right)$ (a) $A, m, D_{0}$ deterministic, and (b) $A, m, D_{0}$ random. 

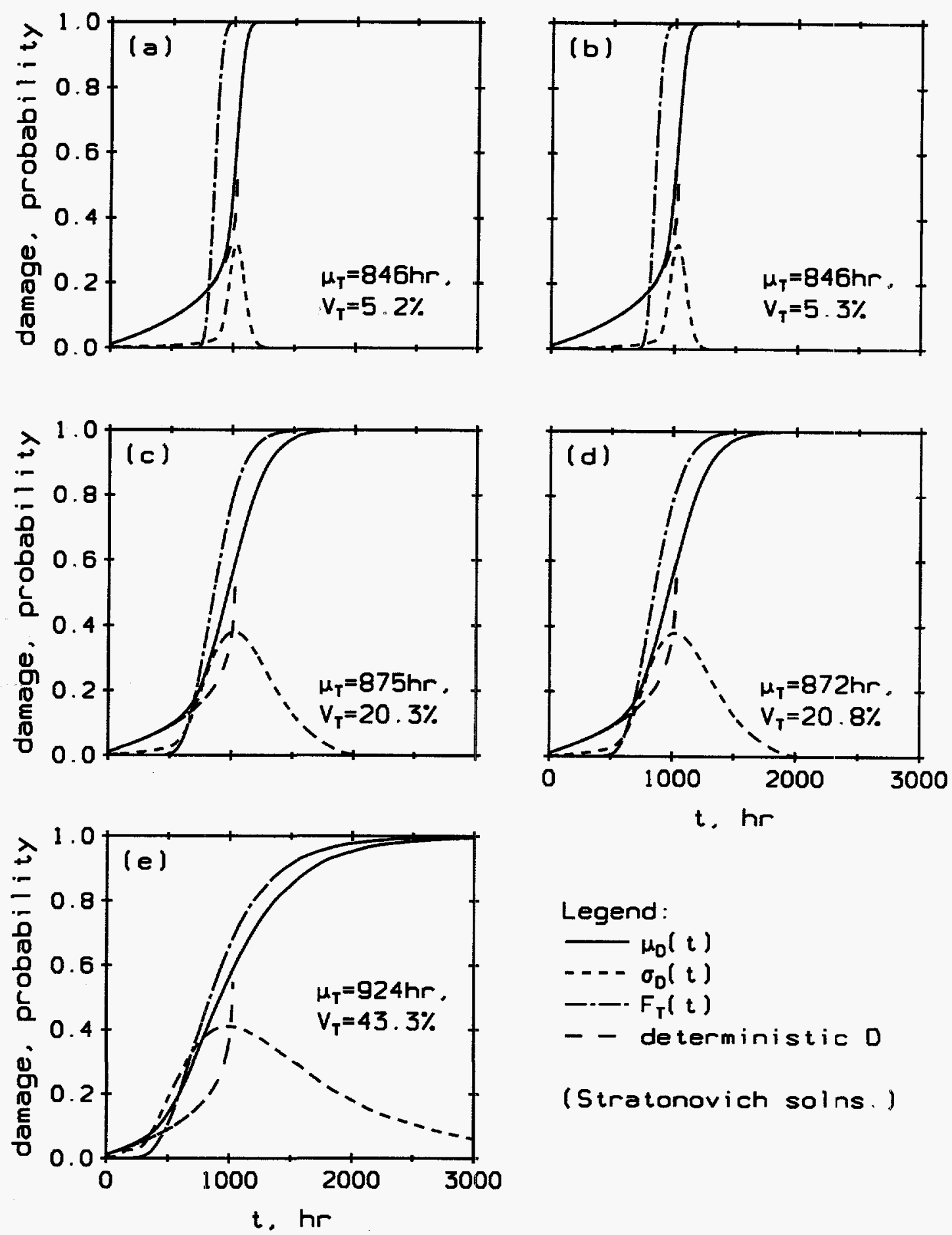

\section{Legend :}

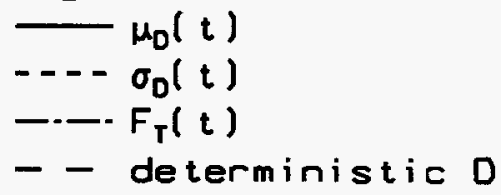

(Stratonovich solns.)

Figure 5.12: Statistics of damage and failure probabilities (under Stratonovich interpretation) in creep of type 316 stainless steel stressed to $199 \mathrm{MPa}$ at $1100^{\circ} \mathrm{F}$ (with $\sqrt{c_{4}} / c_{3}=300 M P a \sqrt{h r}$ ) (a) all deterministic (b) only $D_{0}$ random, (c) $D_{0}, A$ random (d) $D_{0}, A, D_{c}$ random (e) $D_{0}, A, D_{c}, m$ random 

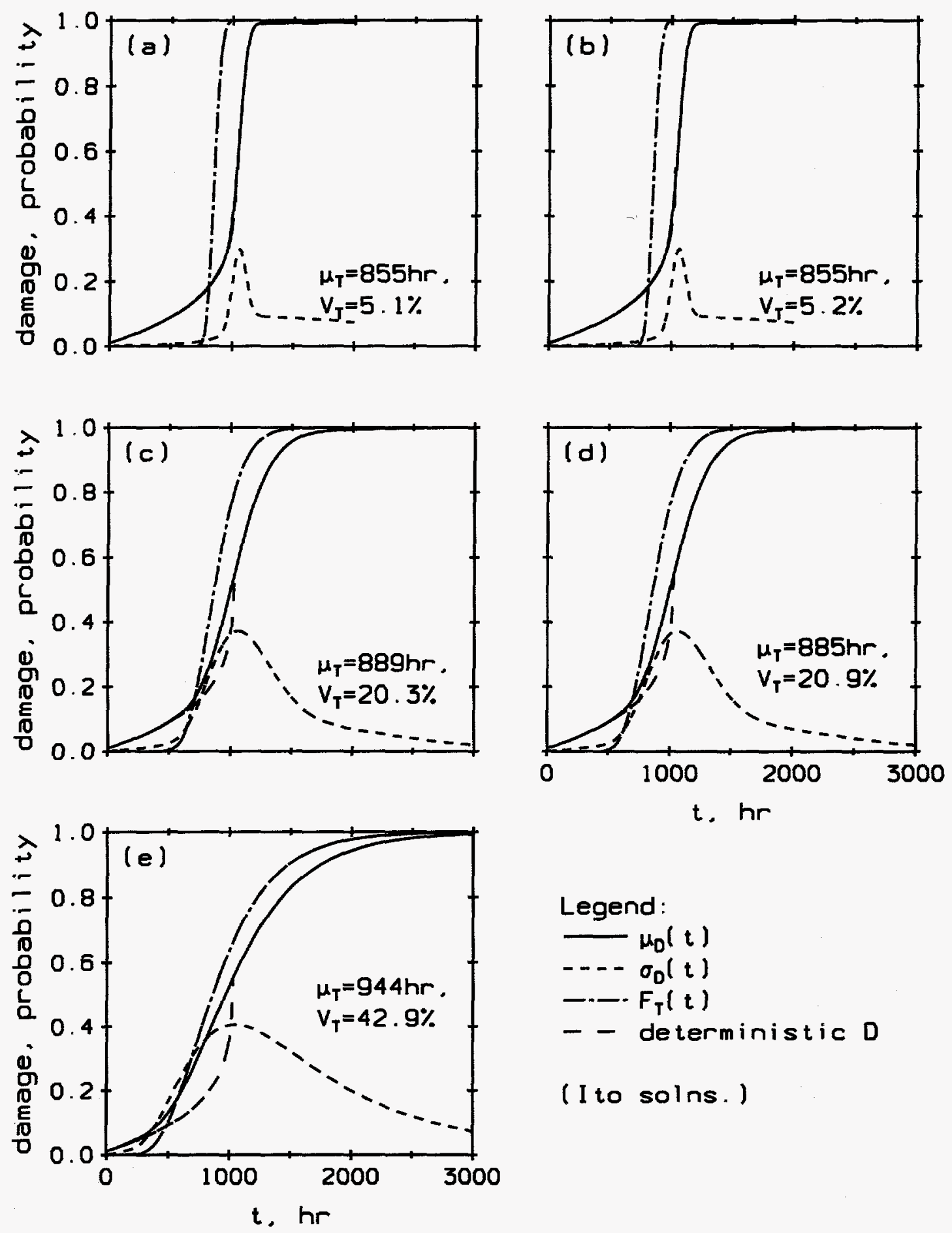

Figure 5.13: Statistics of damage and failure probabilities (under Ito interpretation) in creep of type 316 stainless steel stressed to $199 \mathrm{MPa}$ at $1100^{\circ} \mathrm{F}$ (with $\sqrt{c_{4}} / c_{3}=$ $300 M P a \sqrt{h r}$ ) (a) all deterministic (b) only $D_{0}$ random, (c) $D_{0}, A$ random (d) $D_{0}, A, D_{c}$ random (e) $D_{0}, A, D_{c}, m$ random 


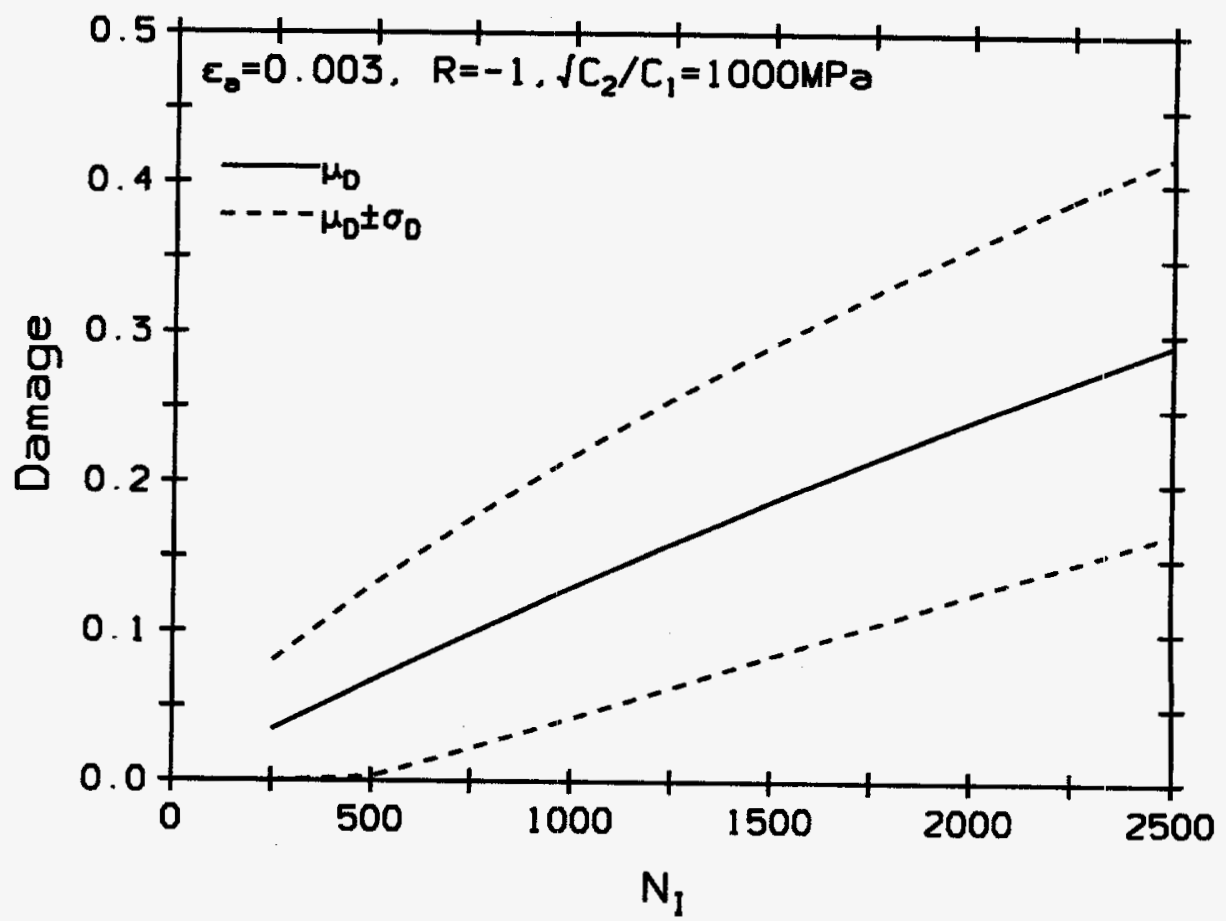

Figure 5.14: Random fatigue damage growth in A106-B carbon steel at $288^{\circ} \mathrm{C}$ in air 


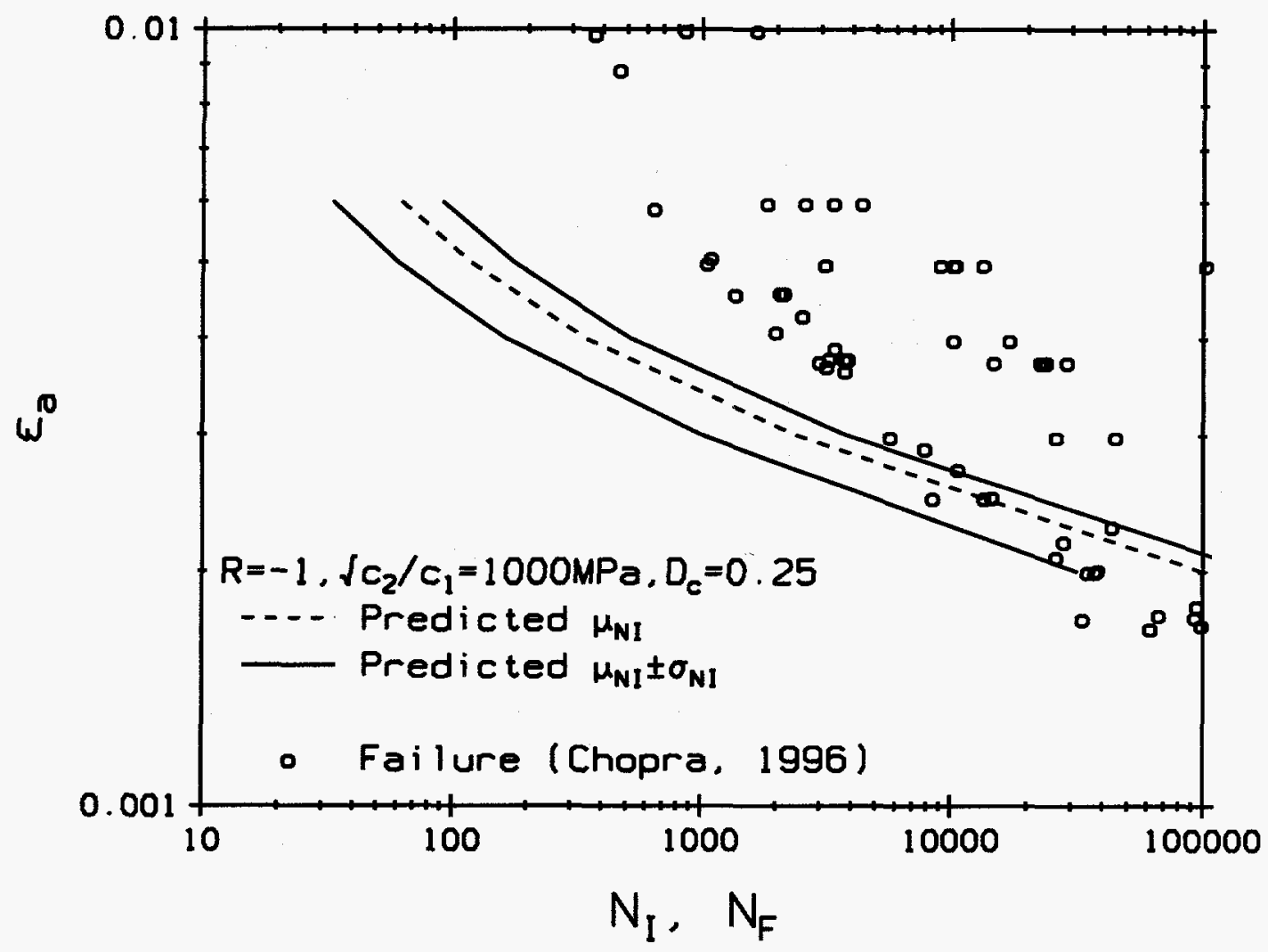

Figure 5.15: Random number of cycles to failure (fatigue crack initiation) in A106-B carbon steel at $288^{\circ} \mathrm{C}$ in air 


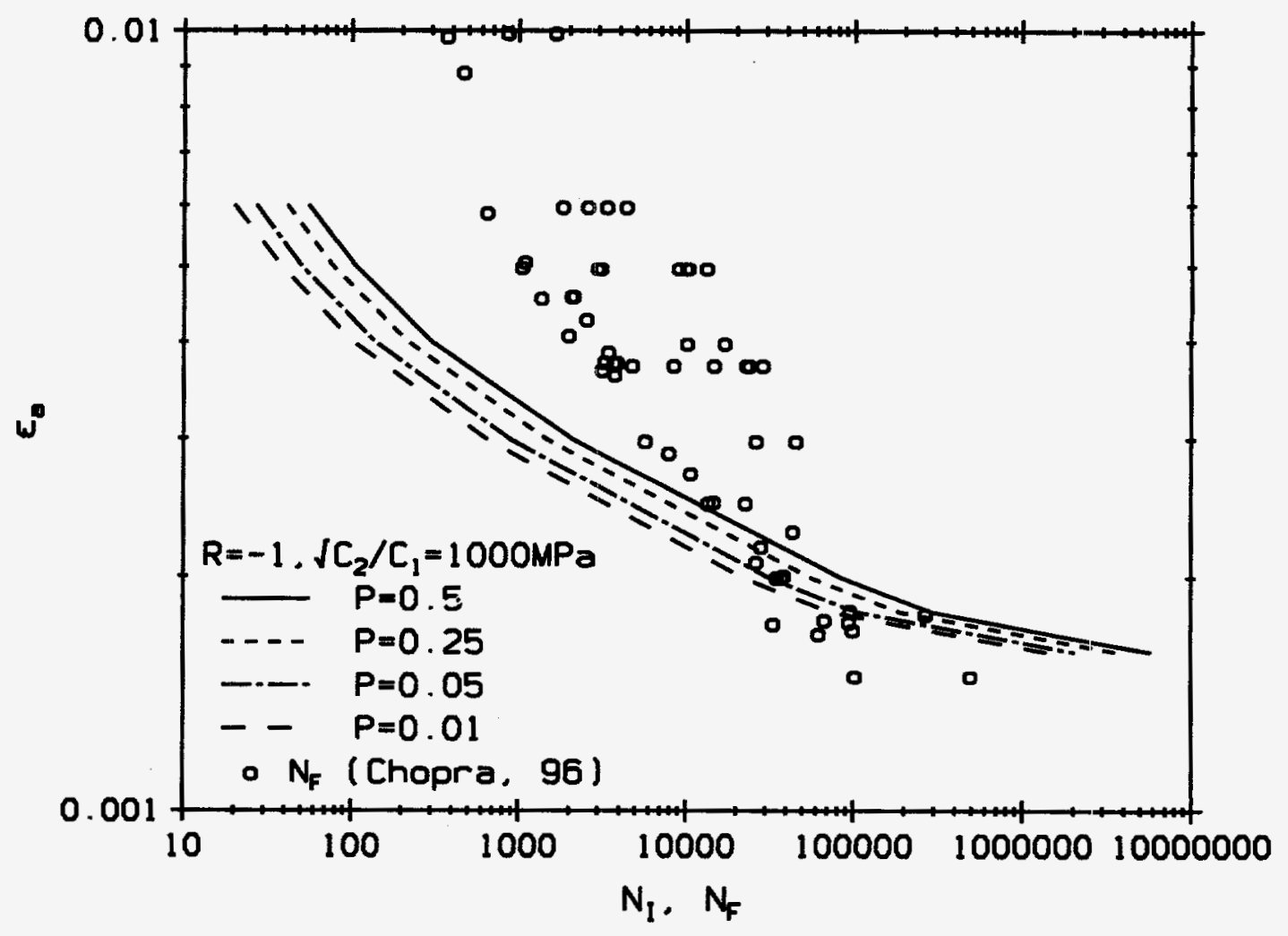

Figure 5.16: Probability of failure (fatigue crack initiation) in A106-B carbon steel at $288^{\circ} \mathrm{C}$ in air 


\section{Chapter 6}

\section{Condition Assessment of Aging Metal Structures}

\subsection{Introduction: Description of Structure}

In the previous chapters, CDM-based formulations of structural deterioration were developed from the fundamental principles of thermodynamics and mechanics. In Chapter 4, using readily available material data, the models were compared to the laboratory test results obtained by other researchers for various grades of steel, aluminum and nickel alloys under conditions of gross inelastic deformation, creep and fatigue. The predictions were found to match the data satisfactorily in most cases. The deterministic models were then extended into the stochastic domain in Chapter 5, where they were shown to be capable of handling material variabilities as well as random noise in damage growth. The purpose of this chapter is to illustrate the applications of the time-dependent condition assessment tools described in Chapters 2 to 5 to existing structures. In particular, the condition assessment and time-dependent reliability of a ring-stiffened steel cylindrical shell, subjected to internal pressurization and weakened by corrosion and thermal creep, will be performed. Such a structure is typical of a nuclear power plant (NPP) steel containment. The approach, however, will be found general enough to apply with minor modifications to steel structures governed by other limit states (e.g., flexural collapse), subject to recurrent extreme loads and suffering from continual deterioration (caused by a combination of general corrosion, fatigue, creep and plastic deformation).

The containment in a NPP is designed using the ASME Code (ASME, 1986) ${ }^{1}$ to withstand accidental pressure build-ups and to limit radiation leakage to the environment. Housed within the containment are the reactor, piping, pumps and valves, steam generators etc. Structurally, steel containments are ring-stiffened thin steel cylindrical shells.

\footnotetext{
${ }^{1}$ Section III Division 1 of the ASME Boiler and Pressure Vessel Code, 1986.
} 
The ASME Code envisages four categories of operating conditions: " $A$ " for normal, " $B$ " for upset, " $C$ " for emergency and " $D$ " for faulted. The Code also classifies stresses produced in the structure into four following types: (i) general primary membrane stress, $P_{m}$ (ii) local primary membrane stress, $P_{L}$ (iii) primary bending stress, $P_{b}$, and (iv) expansion stress, $P_{e}$. Table NE-3217-1 of the Code (ASME, 1986) classifies the stress in a cylindrical or spherical shell due to internal pressure as general primary membrane stress $\left(P_{m}\right)$. Table NE-3221-1 assigns the design stress intensity limit for $P_{m}$ as $1.0 S_{m c}$, in which $S_{m c}=1.1 F_{u} / 4$ is allowable stress and $F_{u}$ is tensile strength. Table I-10.1 lists the value of $S_{m c}$ for SA-516 grade 70 steel, which is commonly used in containments, as $133 \mathrm{MPa}(19.3 \mathrm{ksi})$, up to a temperature of $343^{\circ} \mathrm{C}\left(650^{\circ} \mathrm{F}\right)$. With a $262 \mathrm{MPa}(38 \mathrm{ksi})$ nominal yield strength, this provides a nominal factor of safety of 1.97 at first yield.

The steel containment analyzed in this chapter is idealized as a cylindrical shell of radius $r$ and thickness $h_{0}$ with a hemispherical top also of radius $r$ and thickness $h_{0}$. It has ring stiffeners of cross-sectional area $A_{1}$ placed circumferentially at intervals of $s_{1}$. Vertical stringers of area $A_{2}$ are placed at intervals $s_{2}$ on the cylindrical portion of the shell. The shell has penetrations at different positions to allow for piping and for: access $^{2}$. The shell is fabricated of SA 516 grade 70 steel. Table 6.1 lists the nominal material properties of SA 516/70 steel and the nominal (uncorroded) dimensions of the ring-stiffened steel shell used in the present analysis. The nominal dimensions are based on existing NPP's ( eg Greimann et al, 1982b).

During the past decade, there have been 32 reported cases of containment pressure boundary degradation identified in the 109 commercial nuclear power plants ${ }^{3}$ licensed to operate in the U.S. In particular, corrosion has been detected in the steel containments of 18 existing nuclear power plants (eg, Oland and Naus, 1996; Naus et al, 1996; Shah et al, 1994), where local thickness loss of up to $50 \%$ has been reported in a few instances. In the following sections, the reliability of the NPP steel containment subject to internal pressurization and general corrosion, in the presence of aging of safety-related equipment and thermal creep will be investigated.

\footnotetext{
${ }^{2} \mathrm{~A}$ detailed finite element analysis is required to accurately analyze a shell with stiffeners and discontinuities (eg Cherry, 1996). However, as pointed out by Greimann et al (1982b), a limit analysis is a reasonable and potentially conservative approximation and is deemed sufficient for the purposes of the present analysis.

${ }^{3}$ Out of these, one is under construction and five are under a deferred construction schedule (Naus et al, 1996) as of March 1995.
} 


\subsection{Reliability under Corrosion}

\subsubsection{Effect of Corrosion}

The equation that generally governs design of the ring-stiffened cylindrical steel shell subjected to internal pressurization is (Ellingwood et al, 1996):

$$
S_{m c} \geq D_{n}+L_{n}+P_{n}
$$

where $D_{n}, L_{n}$ and $P_{n}$ are the stresses caused by the nominal dead, live and accidental pressure loads respectively. The subscript $n$ is used to emphasize the nominal values of the variables, which are usually different from their respective mean values. The nominal values and the statistical properties ${ }^{4}$ of the duration of a design-basis event and the peak temperature and pressure attained during such an event are listed in Table 6.1 (Hwang et al, 1983; Ellingwood, 1983). In view of the stringent quality control during the construction of NPP's, the dimensional variabilities are considered insignificant for the present analysis and are treated deterministically in the following.

In an elastic analysis, the circumferential stress in the shell membrane caused by pressurization inside the shell is

$$
P_{n}=P_{a n} \frac{r}{h_{0}}
$$

where $P_{a n}$ is the nominal value of the peak pressure used in the design. The duration of the accidental pressurization is of the order of $10^{3}$ seconds, by which time the secondary safety measures are expected to become operational. Accidental pressurization is a rare event - the estimated rate of occurrence being of the order of $10^{-3} / \mathrm{yr}$ to $10^{-4} / \mathrm{yr}$ ( Hwang et al, 1983) - and therefore, for the purpose of the present analysis, the occurrence of accidental pressures can be modeled as a point process in time when compared to the scale of the service life of interest, which is typically 40 to 60 years. The modeling of the point load process and its stochastic characteristics will be taken up in detail later in this section.

Assuming that the dead and live loads are negligible when the shell is subjected to internal pressure, the design thickness of the shell is (Eqs 6.1 and 6.2):

$$
h_{0}=\frac{P_{a n}}{S_{m c}} r
$$

Using the nominal values listed in Table $6.1, h_{0}=276 \mathrm{kPa} \times 16.8 \mathrm{~m} / 133 \mathrm{MPa}=34.9 \mathrm{~mm}$ $\left(\simeq 1 \frac{3}{8} i n\right)$.

\footnotetext{
${ }^{4}$ Statistical properties of variables concerning design-basis events in a nuclear power plant were obtained using a Delphi, as little or no data exist on such variables. The Delphi is a structured method for achieving group consensus in describing random phenomena in such situations, and has been used successfully in several engineering applications (e.g., Hwang et al, 1983).
} 
The purpose of the NPP containment is to confine radionuclides in the event of an accident, and consequently, the loss of pressure boundary, due either to fracture of the shell or to leakage at penetrations, is defined as failure of the containment. As the shell begins to deform in the event of a pressure build-up, the attachments to the containment fail prior to general shell failure, thanks to the large ductility of carbon steel. Finite element modeling (Greimann et al, 1982b; Cherry, 1996) also suggests that the governing limit state is related to excessive hoop membrane strain or general inelastic deformation of the shell, and not structural collapse. The pressure corresponding to the onset of the critical deformation (or equivalently, the critical strain) is termed the limit pressure, $p_{0}$. Though finite element analyses predict plastic strains at this deformation limit as much as ten times the elastic strains, the limit pressure is conservatively defined as that which produces equal amounts of plastic and elastic strains. The relation between pressure and hoop strain is flat near ultimate because of the ductility of the steel, so the limit pressure is insensitive to the precise value of failure strain. For an axi-symmetric ring-stiffened shell, the limit pressure is expressed as the pressure causing the first yield multiplied by a factor of $\gamma>1$ :

$$
p_{0}=F_{y} \frac{H(t)}{r} \gamma
$$

where $\gamma$ takes into account the plastic deformation of the shell at failure (taken equal to the amount of elastic deformation at yield) and the effects of the stiffeners (Greimann et al, 1982a):

$$
\gamma=\frac{2}{\sqrt{ } 3}+\alpha_{1} ; \quad \alpha_{1}=\frac{A_{1}}{s_{1} H(t)}
$$

$H(t)$ is the thickness of the shell at time $t$ and $\mathrm{r}$ is the radius. The limit state function,

$$
p_{0}-P_{a}(t)=0
$$

can now be written as:

$$
\gamma(t) B \frac{H(t)}{r} F_{y}-P_{a}(t)=0
$$

where $F_{y}$ is the random yield stress and $P_{a}$ is the random peak accidental pressure. The implicit time-dependence of $p_{0}$ and $\gamma$ is shown in eq (6.7), since the shell thickness, $H(t)$, decreases in time on account of general corrosion of the steel. The random variable $B$ is introduced to account for modelling error in formulating the limit state. The limit state (eq 6.6) may also depend on the CDM-based damage function, $D(t)$, which takes into account the service histor $y^{5}$ prior to the event of an internal pressurization.

The shell thickness, $H(t)$, is a random process on account of the random corrosion loss, given by (eq 2.20)

$$
H(t)= \begin{cases}h_{0}-C\left(t-T_{I}\right)^{M} ; & t>T_{I} \\ h_{0} ; & t \leq T_{I}\end{cases}
$$

\footnotetext{
${ }^{5}$ Potential damage-causing scenarios during the service life may include arc strikes, large impacts, periodic leakage rate tests and so on, which may introduce residual stresses etc.
} 
in which $C$ is the random rate, $M$ is the random exponent and $T_{I}$ is the random initiation time. Laboratory and field data on these random variables (for steel), when available, are found to depend strongly on the environmental and physical conditions. Since it is not known a priori what kind of corrosive environment may exist at the critical location of a particular NPP containment ${ }^{6}$, different sets of statistics for these random variables (based on available data compiled and analysed by Ellingwood et al, 1996) have been adopted later in this section for reliability and sensitivity studies. Broadly, three different scenarios have been considered: "severe" corrosion, "moderate" corrosion and no corrosion, along with a situation where the corrosion induction period is absent. These different scenarios and parameters are listed in Table 6.2.

Let us now introduce two non-dimensional random variables, $X$ and $Y$, - the normalized yield strength and the normalized peak accidental pressure magnitude respectively:

$$
X=\frac{F_{y}}{F_{y n}} ; \quad Y=\frac{P_{a}}{P_{a n}}
$$

The statistical properties of $X$ and $Y$ are listed in Table 6.1. Because of the simplicity of the limit state, the uncertainty in $B$ is subsumed in $X^{7}$. The limit state equation (6.7) can then be normalized by the design equation (6.3) and expressed in terms of the dimensionless random variables, $X$ and $Y$ :

$$
\gamma(t) \frac{F_{y n}}{S_{m c}} X \frac{H(t)}{h_{0}}-Y(t)=0
$$

As noted previously, the occurrence of the events leading to accidental pressure loads can be modeled as a point process in time. The underlying physical process of events leading to a pressure build-up can be broadly grouped into (i) pure "chance" phenomena, like human error, the frequency of which remains essentially constant in time, and (ii) "aging" phenomena that might cause mechanical or electrical support systems either to malfunction or to be unavailable when needed to mitigate an accident. The propensity of the latter may increase with time in the absence of corrective intervention ${ }^{8}$. The first (chance) process is described by $N_{0}(t)$, while the second (aging) process is described by $N_{A}(t)$. Let the mean rate of occurrence of the "chance" process be $\lambda_{0}$, and let the mean rate of occurrence of the "aging" process be denoted by $\lambda_{A}(t)$. Since aging involves a lack of self-healing abilities, $\lambda_{A}(t)$ is a non-decreasing function of time.

\footnotetext{
${ }^{6}$ Significant corrosion caused by leaked borated water has been detected in some locations in existing nuclear power plants. Corrosion loss may be aggravated by accumulation of water or condensation of leaked steam on pressure-retentive structures in a containment. The relative humidity inside containments may range up to $60 \%$ (Oland and Naus, 1996) which is high enough to accelerate corrosion at service temperature.

${ }^{7}$ In the illustrations herein, the uncertainty in modelling error $B$ is small because of the simplicity of the limit state considered. In a more comprehensive analysis, its characteristics would depend on the nature of the finite element analysis and level of refinement in modelling.

${ }^{8}$ Previous studies of time-dependent reliability of steel containments have not considered this aspect of load occurrence. The purpose of including this in the present anaiysis is to see if an increasing rate of occurrence of design-basis events has a significant impact on the reliability of this and similar structures.
} 
Let us now make some assumptions about the point processes, $N_{0}(t)$ and $N_{A}(t)$. Obviously, $N_{0}(0)=0$ and $N_{A}(0)=0$. It is assumed that if there were to be an accidental event during the service life, any equipment necessary would be repaired or replaced, so that the cause of the present event would have no bearing on future events. Then, the number of occurrences in disjoint intervals of time are independent for both processes, i.e., for $p<q<s<t, N_{0}(t)-N_{0}(s)$ is statistically independent of $N_{0}(q)-N_{0}(p)$, and $N_{A}(t)-N_{A}(s)$ is statistically independent of $N_{A}(q)-N_{A}(p)$. Also, since the aging and chance processes have different origins, it is reasonable to assume that $N_{0}(t)$ and $N_{A}(t)$ are statistically independent at all $t$. Then the point processes $N_{A}(t)$ and $N_{0}(t)$ are independent Poisson processes, and their sum, $N(t)=N_{A}(t)+N_{0}(t)$, which represents the overall occurrence of accidental pressures, is also a Poisson process with mean rate of occurrence

$$
\lambda(t)=\lambda_{0}+\lambda_{A}(t) .
$$

$\lambda(t)$ is a non-decreasing function of time. A Poisson process is called homogeneous (or uniform) if its rate function is constant in time. As can be seen from the above, in the presence of aging, the occurrence of internal pressurization is a non-homogeneous Poisson process.

The rate function, $\lambda(t)$, of a Poisson process is related to the probability density function, $f_{\tau}\left(s \mid t_{0}\right)$, of the event inter-arrival time, given the previous occurrence instant as $t_{0}$ :

$$
f_{\tau}\left(s \mid t_{0}\right)=\lambda\left(t_{0}+s\right) \exp -\left[\Lambda\left(t_{0}+s\right)-\Lambda\left(t_{0}\right)\right]
$$

where $\Lambda(t)$ is the mean of the Poisson process:

$$
\Lambda(t)=\int_{0}^{t} \lambda(v) d v
$$

with the initial time taken as 0 . The conditional density of the occurrence time, $T^{*}$, in the interval $\left[t_{1}, t_{2}\right]$ is also a function of the arrival rate (Snyder and Miller, 1991):

$$
f_{T^{*}}\left(t^{*}\right)= \begin{cases}\frac{\lambda\left(t^{*}\right)}{\int_{t_{1}}^{t_{2}} \lambda(v) d v} ; & t_{1} \leq t^{*} \leq t_{2} \\ 0 ; & \text { otherwise }\end{cases}
$$

Given that there are $n$ occurrences of a Poisson process in the interval $[0, t]$, the conditional joint density of the ordered occurrence times, $\underline{T}^{*}$, with $0 \leq T_{1}^{*} \leq T_{2}^{*} \ldots T_{n}^{*} \leq t$, is

$$
f_{T^{*}}\left(\underline{t^{*}} \mid N(t)=n\right)=\prod_{i=1}^{n}\left[\frac{\lambda\left(t_{i}^{*}\right)}{\int_{0}^{t} \lambda(v) d v}\right]
$$

which will be used in the reliability formulation in the following.

The degradation processes due to corrosion are assumed to occur slowly enough that; the time-dependent resistance variables $\left(\gamma(t), H(t) / h_{0}\right)$ can be treated as constants during the duration of an accidental pressurization (Mori, 1992). For brevity, let $R(t)$ denote 
the random process representing the resistance (eq 6.10)

$$
R(t)=\gamma(t) S_{F} X \frac{H(t)}{h_{0}}
$$

and let $r(t)$ denote one realization of the random process. The reliability of the shell at time $t$, given $n$ occurrences of accidental pressurization at known times $t_{1}^{*}, \ldots, t_{n}^{*}$ (in increasing order), can then be expressed as

$$
\mathrm{P}\left[T_{f}>t \mid N(t)=n, \underline{T}^{*}=\underline{t}^{*}\right]=\mathrm{P}\left[\cap_{i=1}^{n} R\left(t_{i}^{*}\right)>Y\left(t_{i}^{*}\right)\right]
$$

where $T_{f}$ is the random time to failure. Fixing the values of the random variables $X, C, T_{I}, M$ in addition to fixing the number and time instants of load occurrences, the conditional reliability can be expressed as

$$
L(t \mid A)=\mathrm{P}\left[T_{f}>t \mid A\right]=\mathrm{P}\left[\cap_{i=1}^{n} Y\left(t_{i}^{*}\right) \leq r\left(t_{i}^{*}\right)\right]
$$

where the event $\{A\}$ is

$$
\{A\}=\left\{N(t)=n, \underline{T}^{*}=\underline{t}^{*}, X=x, C=c, M=m, T_{I}=t_{I}\right\}
$$

Assuming that the peak pressures occurring at different times are statistically independent,

$$
L(t \mid A)=\mathrm{P}\left[T_{f}>t \mid A\right]=\cap_{i=1}^{n} F_{Y\left(t_{i}^{*}\right)}\left[r\left(t_{i}^{*}\right)\right]
$$

Further assuming that the peak pressures are identically distributed, the conditional reliability simplifies to

$$
L(t \mid A)=\mathrm{P}\left[T_{f}>t \mid A\right]=\prod_{i=1}^{n} F_{Y}\left[r\left(t_{i}^{*}\right) \mid A\right]
$$

where the common probability distribution of the peak pressures is $F_{Y}(\cdot)$ (defined by Table 6.1).

We now remove the conditioning on the occurrence times of the $n$ loads. Defining the new conditioning event by

$$
\left\{A_{1}\right\}=\left\{N(t)=n, X=x, C=c, M=m, T_{I}=t_{I}\right\}
$$

the reliability at time $t$ is (using the theorem of total probability and eq 6.15):

$$
\begin{aligned}
L\left(t \mid A_{1}\right) & =\int_{0}^{t} \int_{0}^{t} \ldots \int_{0}^{t} \prod_{i=1}^{n} F_{Y}\left[r\left(t_{i}^{*}\right) \mid A\right] f_{\underline{T^{*}}}\left[\underline{t^{*}} \mid N(t)=n\right] d \underline{t}^{*} \\
& =\int_{0}^{t} \int_{0}^{t} \ldots \int_{0}^{t} \prod_{i=1}^{n} F_{Y}\left[r\left(t_{i}^{*}\right) \mid A\right] \prod_{i=1}^{n}\left[\frac{\lambda\left(t_{i}^{*}\right)}{\int_{0}^{t} \lambda(v) d v}\right] d t_{1}^{*} d t_{2}^{*} \ldots d t_{n}^{*} \\
& =\left(\int_{0}^{t} F_{Y}[r(s) \mid A]\left[\frac{\lambda(s)}{\int_{0}^{t} \lambda(v) d v}\right] d s\right)^{n}
\end{aligned}
$$


where the Poisson arrival rate is given by eq (6.11). Next, the conditioning on the number of loads is removed. Defining the new conditioning event by

$$
\left\{A_{2}\right\}=\left\{C=c, M=m, T_{I}=t_{I}\right\}
$$

the reliability at time $t$ (using the theorem of total probability) is

$$
\begin{aligned}
L\left(t \mid A_{2}\right) & =\sum_{n=0}^{\infty}\left(\int_{0}^{t} F_{Y}[r(s) \mid A]\left[\frac{\lambda(s)}{\int_{0}^{t} \lambda(v) d v}\right] d s\right)^{n} \mathrm{P}[N(t)=n] \\
& =\sum_{n=0}^{\infty}\left(\int_{0}^{t} F_{Y}[r(s) \mid A]\left[\frac{\lambda(s)}{\int_{0}^{t} \lambda(v) d v}\right] d s\right)^{n} \frac{\exp [-\Lambda(t)] \Lambda(t)^{n}}{n !} \\
& =\exp \left[-\Lambda(t)\left(1-\int_{0}^{t} F_{Y}[r(s) \mid A]-\frac{\lambda(s) d s}{\int_{0}^{t} \lambda(v) d v}\right)\right]
\end{aligned}
$$

which reduces to the result obtained by Ellingwood and Mori (1993) if the Poisson process is homogeneous, i.e., $\lambda(t)=\lambda_{0}$ and $\Lambda(t)=\lambda_{0} t$. Finally, if the random variables $X, C, M, T_{I}$ are statistically independent, their joint density is the product of their marginals, and the unconditional reliability can be expressed as (once again using the theorem of total probability):

$$
\begin{gathered}
L(t)=\int_{x} \int_{c} \int_{m} \int_{t_{I}} \exp \left[-\Lambda(t)\left(1-\int_{0}^{t} F_{Y}[r(s) \mid A] \frac{\lambda(s) d s}{\int_{0}^{t} \lambda(v) d v}\right)\right] \times \\
f_{X}(x) f_{C}(c) f_{M}(m) f_{T_{I}}\left(t_{I}\right) d x d c d m d t_{I}
\end{gathered}
$$

where $r(t)$ is given by eq (6.16). In deriving Eq (6.26), the only assumptions made regarding the statistical characteristics of the load process are that the loads occur according to a (nonhomogeneous) Poisson point process and their peak magnitudes are statistically independent and identically distributed.

We now make further assumptions about the "aging" process and determine the rate $\lambda_{A}(t)$ and the conditional density function of its occurrence times. It is clearly seen that the inter-arrival time, $\tau$, between two successive occurrences of events in the aging process, $N_{A}(t)$, is simply the time to failure of the equipment after its installation. The life-time of equipment has frequently been modeled in the existing literature by the Weibull distribution (e.g., Lawless, 1982). The two-parameter Weibull distribution 
is versatile enough to represent many different aging situations. Adopting the Weibull model for the time to failure of equipment, the probability distribution function of $\tau$ is

$$
F_{\tau}(t)=1-\exp \left[-(t / u)^{\alpha}\right]
$$

where $\alpha$ is the shape parameter and $u$ is the scale parameter. The coefficient of variation (COV) of the time to failure is a function of the shape parameter:

$$
V_{\tau}=\sqrt{\frac{\Gamma(1+2 / \alpha)}{\Gamma^{2}(1+1 / \alpha)}-1}
$$

and the scale parameter can be determined from the mean failure time, $\mu_{\tau}$ :

$$
u=\frac{\mu}{\Gamma(1+1 / \alpha)}
$$

If the time to failure is described by a Weibull distribution, the rate function of the aging process is,

$$
\lambda_{A}(t)=\frac{\alpha}{u}\left(\frac{t}{u}\right)^{\alpha-1}
$$

and the conditional density of the occurrence time as

$$
f_{T}^{*}\left(t^{*}\right)=\alpha \frac{t^{* \alpha-1}}{t^{\alpha}}
$$

When $\alpha<1$, the Poisson arrival rate decreases with time. At $\alpha=1, \lambda_{A}(t)$ is constant, making the distribution of $\tau$ exponential and the conditional distribution of the occurrence time uniform. For $\alpha>1$, the rate of occurrence increases with time and represents a realistic "aging" process. A "linear failure rate" model (implying $\alpha=2$ ) has been suggested in some studies of aging of certain mechanical and electrical equipment (e.g., Vesely, 1987).

Several cases of the aging process have been used in the reliability study in the following subsection to determine whether the time- dependent reliability analysis and condition assessment might be sensitive to this effect. Values of $\alpha$ less than 1 have not been considered as they lead to decreasing rates of failure with time. The case of no equipment aging has also been considered to establish a baseline for "chance" failure alone. Table 6.3 lists the different values of the parameters $\alpha$ and $\mu$ used in the analyses along with the equipment failure probability at the end of a postulated service life of 60 years in each case.

\subsubsection{Numerical Examples and Sensitivity Analyses}

We now present numerical results from the reliability analyses of the steel cylindrical shell against pressurization under different cases of corrosion and equipment aging. Reliabilities are computed up to a projected service life of 60 years. Two different chance 
occurrence rates have been considered in the Poisson pulse process: one representing a "low" chance failure rate, $\lambda_{0}=0.0001 / y r$, and the other representing a "high" chance failure rate - twenty times higher - with $\lambda_{0}=0.002 / y r$. The aging of the safety equipment is assumed to follow the Weibull distribution (eq 6.30) and the total Poisson arrival rate is given by eq (6.11). Table 6.3 lists the different aging situations.

The random variables $X, C, M$ and $T_{I}$ are assumed statistically independent (eq 6.26). As listed in Table 6.1, the peak accidental pressure magnitude has the Type I distribution of largest values and the yield stress has the Lognormal distribution. The statistics of $X$ and $Y$ have been taken from Ellingwood et al (1996). The corrosion parameters $C$ and $T_{I}$ (eq 6.8) are lognormally distributed and the exponent, $M$ is normally distributed ${ }^{9}$. Three different corrosion scenarios have been envisaged (based on Ellingwood et al, 1996) and the corresponding sets of parameters are shown in Table 6.2. The section loss at 60 yrs due to severe corrosion evaluated at the mean values of $C, T_{I}$ and $M$ is $9 \mathrm{~mm}(0.36 \mathrm{in})$, which is about $27 \%$ of the original thickness; and in case of moderate corrosion the loss is $3.6 \mathrm{~mm}(0.14 i n)$ which is about $10 \%$ of $h_{0}$.

Eq (6.26) may be abbreviated as,

$$
L(t)=\int_{\underline{z}} l(\underline{z}) f_{\underline{Z}}(\underline{z}) d \underline{z}
$$

where $\underline{Z}$ is the random vector $\left[X, C, M, T_{T}\right]^{\prime}$. The kernel, $l(\underline{z})$, is evaluated by subdividing the interval $[0, t]$ and using a 21-point Gauss-Kronrod quadrature (double precision subroutine DQDAGS from IMSL, 1991) for each subinterval and comparing the error for each subinterval with a 10 -point Gauss quadrature rule subject to certain maximum allowable relative and absolute errors. Monte-Carlo simulations have been used to obtain the realizations of $\underline{z}$. The reliability estimate, $\hat{L}(t)$, and its variance are given by

$$
\begin{aligned}
\hat{L}(t) & =\frac{1}{N_{M C T}} \sum_{i=1}^{N_{M C T}} l_{i}(\underline{z}) \\
\operatorname{var}[\hat{L}(t)] & =\frac{1}{N_{M C T}}\left[\frac{\sum l_{i}^{2}}{N_{M C T}}-\{\hat{L}(t)\}^{2}\right]
\end{aligned}
$$

where $N_{M C T}$ is the number of Monte-Carlo trials. The coefficient of variation of the estimate was kept under $10 \%$ in each case by using a sufficient number of trials (which ranged from 50,000 to $1,000,000$ ). It is more convenient to plot reliability in terms of its complement, the cumulative failure probability (CFP) given by

$$
F_{T_{f}}(t)=\mathrm{P}\left[T_{f} \leq t\right]=1-L(t)
$$

\footnotetext{
${ }^{9}$ The process of general corrosion is diffusion dominated. In a purely diffusion controlled process, the exponent is equal to $1 / 2$, and deterministic values around $2 / 3$ are often reported in the literature. Consequently, the mean of $M$ is taken to be 0.7 in the present analysis.
} 
where $T_{f}$ is the random time to failure of the structure. The figures in this subsection show CFP's as functions of time. Plots of failure probabilities in all figures of this subsection are drawn to the same scale to facilitate the comparison of different scenarios.

Figs 6.1(a)-(c) show the Poisson arrival rate, $\lambda(t)$, for the different aging situations and compare them with the chance failure rate. The square-root rate $(\alpha=1.5)$ is seen to be the most severe, but not significantly different from the linear case. Figs 6.2(a)(c) show the corresponding CFP's for the case of "severe" corrosion. It is observed that ignoring the equipment aging phenomenon may lead to under-prediction of $F_{T_{f}}(t)$. For example, ignoring the aging effect when equipment failure probability is $3 \%$ at 60 years, causes an order of magnitude under-prediction of CFP at 60 years. Whether this difference is significant for risk-informed decision making should be considered in the context of other uncertainties in probabilistic risk assessment (Lehner et al, 1997).

The effect of ignoring corrosion is presented in Figures 6.3(a)-(c) corresponding to the arrival rates shown in Figs 6.1(a)-(c). The rate of increase in CFP when corrosion is ignored is much slower, and at 60 years it is more than four orders of magnitude lower than the CFP when severe corrosion is assumed. Therefore, it is important to obtain good estimates of corrosion loss. This presents a problem when areas susceptible to corrosion loss are inaccessible for inspection.

The mean failure time of safety equipment apparently has an appreciable effect on the reliability of the structure (e.g., there is a difference of two orders of magnitude in $F_{T_{f}}(60)$ for $\mu=120 \mathrm{yr}$ and $\left.\mu=\infty\right)$, but the precise value of $\alpha$ has a secondary importance for a given mean failure time. Also, a $\mu$ greater than about $10 t_{L}$ can qualify as infinite life (especially with a high $\alpha$ ), in which case ignoring the aging effects of equipment is acceptable. Thus it is important to know the life expectancy of the primary safety equipment, but the precise value of its scatter is less essential. In view of this, the scale parameter is fixed at $\alpha=2$ in the following; this results in a linear Poisson rate function, which would be easier to fit to a limited data.

Fig 6.4(a) summarizes the effects of severe, moderate and zero corrosion with linear $\lambda(t)$. Ignoring corrosion entirely can cause more than four orders of magnitude underprediction of the CFP at $60 \mathrm{yr}$ if the actual rate of corrosion is "severe". Even for "moderate" corrosion (which causes only about $10 \%$ mean section loss after $60 y r$ ) the extent of under-prediction is found to be more than 2 orders of magnitude at the end of the projected service life. Fig 6.4(b) repeats the above analysis when the chance occurrence rate is increased to $\lambda_{0}=0.002 / y r$. It is observed that if equipment aging is the dominant cause of internal pressurization, then the background chance occurrence rate has little impact on risk. On the other hand, if chance occurrences of pressurization dominate, then a twenty times increase in $\lambda_{0}$ causes a like increase in the CFP.

The effects of ignoring the variability in the corrosion initiation time, and ignoring the initiation time altogether are illustrated in Figs 6.5(a) and (b). The former shows the effect of treating $T_{I}=10 y r$ deterministically while the latter shows the effect of 
setting $T_{I}=0$ under identical situations. It is clearly seen that setting $T_{I}$ equal to its mean value makes little difference to the reliability, but reduces the dimension of the random vector, $\underline{Z}$, by one. Ignoring the initiation time entirely, however, leads to a more pessimistic prediction of reliability. The difference can be as high as an order of magnitude but decreases with increasing time. Therefore, $T_{I}$ should be included in the reliability formulation, but an estimate of its mean is sufficient, and its variability need not be considered in time-dependent reliability assessment.

The effects of treating $C$ and $M$ deterministically are illustrated in Figures 6.6 and 6.7. As can be expected, treating the rate deterministically is shown to under-predict the cumulative failure probability, but not severely (roughly by a factor of 2). What is less obvious is that treating the exponent deterministically can cause under-prediction by as much as two orders of magnitude. This observation is significant in light of the fact that the exponent usually is treated as constant.

The following general conclusions can be drawn from the above analysis:

1. Uniform corrosion of the containment steel shell should not be neglected in reliability analyses and condition assessment, even when it is expected to be moderate.

2. When corrosion is considered, it is advisable to consider it as a random process. The randomness in the corrosion initiation time may be neglected, but the corrosion rate and the exponent should both be treated as random.

3. Since increases in the rate of occurrence of potential design-basis events caused by equipment aging apparently has some impact on the time dependent reliability, a study should be undertaken to determine whether current equipment maintenance programs might inadvertently cause such increases. Frequent testing of safetyrelated equipment may actually have an adverse effect on their performance during a design-basis event. An examination of this issue, of course, is outside the scope of this research.

\subsection{Reliability under Thermal Creep Damage}

Accidental pressurization in a containment would be accompanied by a rise in temperature (Hwang et al, 1983). Though the peak pressure and the peak temperature do not necessarily occur simultaneously, they can have a sufficient overlap to cause creep straining of the structure. In this section, the reliability of the idealized steel containment under thermal creep damage accumulation will be investigated. The formulation of stochastic creep damage growth from section 5.5 will be used for this purpose. The effects of corrosion and equipment aging will also be included in the creep damage growth model.

The assumptions made about the occurrence of events in the previous section are 
continued here: They are rare events with durations infinitesimally small compared to the life-time of the structure, and can be modelled as a non-homogeneous Poisson pulse processes. One of the consequences of the above assumptions is that the corroded depth of a section can be treated as constant over the duration of such loads. Two general conclusions from section 6.2.2 about the corrosion and aging processes, namely, the initiation time can be fixed at its mean value and occurrence rate of the aging process increases linearly, will be adopted here to reduce the dimensionality of the problem.

\subsubsection{Creep Damage at Elevated Temperatures}

The following assumptions (based on the data available in Hwang et al, 1983; Ellingwood, 1983) are made about the temperature and pressure histories during the course of a design-basis event. The temperature and pressure within the containment rise in a very short time $(\sim 10 \mathrm{sec})$ to their peak values, $P_{a}$ and $\theta_{a}$ respectively, and remain steady at the peak values for a duration of $\Delta t(\sim 20 \mathrm{~min})$. The pressure drops off relatively quickly after $\Delta t$ and attains the base value ${ }^{10}$ in less than one day. The temperature however takes longer ( $\sim 11$ days) to return to its base value $\left(\sim 120^{\circ} \mathrm{F}\right)$. The peak pressures and temperatures in events occurring at different times are assumed to be statistically independent and identically distributed. Statistical dependence, however, may be expected between $P_{a}$ and $\theta_{a}$ during a single event. No statistical dependence is assumed to exist between the temperature and pressure in events occurring at different times. The statistics of $P_{a}, \theta_{a}$ and $\Delta t$ are given in Table 6.1. In the event of a pressure and temperature build-up, creep damage may be envisaged to grow during a time-interval $\Delta t$, at the constant ${ }^{11}$ temperature $\theta_{a}$ under the action of the constant load $P_{a}$. The action of the load appears in the form of the membrane stress; this depends on the corrosion loss and thus on the time of occurrence of the event.

Suppose that $n$ loads occur during the life of the structure at random instants of time, $t_{i}(i=1,2, \ldots, n)$. The damage, $\left.D\left(\Delta t_{i}\right)\right)$, at the end of the $i$ th load occurrence may be expressed as (eq 5.89)

$$
\left(1-D\left(\Delta t_{i}\right)\right)^{m_{i}+1}=\left(1-D\left(\Delta t_{i-1}\right)\right)^{m_{i}+1}-A_{1 i}\left(m_{i}+1\right) \Delta t_{i}-B_{1_{i}}\left(m_{i}+1\right) W\left(\Delta t_{i}\right)
$$

where $W\left(\Delta t_{i}\right)$ is the increment in the standard Wiener process during the interval $\Delta t_{i}$. The time and temperature dependent coefficients,

$$
\begin{aligned}
& A_{1_{i}}=\frac{4}{3} \frac{A_{i}}{\sigma_{f_{i}}} \sigma_{\infty_{i}}^{m_{i}+1} \\
& B_{1_{i}}=\frac{4}{3} \frac{A_{i}}{\sigma_{f_{i}}} \sigma_{\infty_{i}}^{m_{i}}\left(\sqrt{c_{4}} / c_{3}\right)_{i}
\end{aligned}
$$

\footnotetext{
${ }^{10}$ The pressure within a NPP containment is maintained at a constant level, which could be slightly above or below the atmospheric pressure depending on the type of the plant (Oland and Naus, 1996).

${ }^{11}$ Constant in any given realization of the process.
} 
where $\sigma_{f}$ is the true failure stress, $A$ and $m$ are the creep power law parameters and $\left(\sqrt{c_{4}} / c_{3}\right)$ is the noise intensity. The subscript $i$ denotes their values at temperature $\theta_{a_{i}}$. The far-field stress,

$$
\sigma_{\infty_{i}}=P_{a_{i}} \frac{r}{H_{i}}
$$

depends on the shell radius, $r$, the pressure $P_{a_{i}}$ and the shell thickness, $H_{i}$ at time $t_{i}$.

The form of eq (6.36) suggests a recursive relation which would allow the final creep damage, $D_{n}$, to be written in terms of the initial damage, $D_{0}$. The algebraic expression can be considerably simplified if the exponent $m_{i}$ can be assumed to remain constant over the range of temperature attainable in an NPP containment. In this case,

$$
\left(1-D_{n}\right)^{m+1}=\left(1-D_{0}\right)^{m+1}-\sum_{i=1}^{n} A_{2_{i}} \sigma_{\infty_{i}}^{m+1} \Delta t_{i}-\sum_{i=1}^{n} B_{2_{i}} \sigma_{\infty_{i}}^{m} W\left(\Delta t_{i}\right)
$$

where $A_{2 i}$ and $B_{2_{i}}$ depend only on $\theta_{a_{i}}$ :

$$
\begin{aligned}
A_{2_{i}} & =\frac{4}{3} \frac{A\left(\theta_{a_{i}}\right)}{\sigma_{f}\left(\theta_{a_{i}}\right)}(m+1) \\
B_{2_{i}} & =\frac{4}{3} \frac{A\left(\theta_{a_{i}}\right)}{\sigma_{f}\left(\theta_{a_{i}}\right)}(m+1)\left(\sqrt{c_{4}} / c_{3}\left(\theta_{a_{i}}\right)\right)
\end{aligned}
$$

Failure ${ }^{12}$ occurs when the accumulated damage exceeds the critical damage, $D_{c}$. The conditional cumulative failure probability is

$$
F_{T_{f}}\left(t_{L} \mid \underline{\Omega}, N\left(t_{L}\right)=\dot{n}, t_{i}^{*}\right)=1-\Phi\left[\frac{\left(1-D_{0}\right)^{m+1}-\left(1-D_{c}\right)^{m+1}-\sum_{i=1}^{n} A_{2_{i}} \sigma_{\infty_{i}}^{m+1} \Delta t_{i}}{\left(\sum_{i=1}^{n} B_{2_{i}}^{2} \sigma_{\infty_{i}}^{2 m} \Delta t_{i}\right)^{1 / 2}}\right]
$$

where the statistical independence of the Wiener increments have been used. The vector, $\underline{\Omega}=\left\{\underline{\theta}_{a}, \underline{P}_{a}, \underline{\Delta t}, \underline{H}, D_{0}, D_{\mathrm{c}}\right\}$, where $\underline{\theta}_{a}, \underline{P}_{a}, \underline{\Delta t}, \underline{H}$ are each vectors of size $n$. The $n$ loads are assumed to occur at fixed instances $t_{i}^{*}$. To find the unconditional failure probability, it is convenient to remove the conditioning on the $t_{i}^{*}$ 's first, which are distributed according to eq (6.14) with $t_{i}^{*}$ and $t_{j}^{*}$ statistically independent for $i \neq j$. The conditioning on the corrosion parameters, the temperatures and pressures, and the duration may be removed subsequently. Finally, the conditioning on the number of loads can be removed as

$$
F_{T_{f}}\left(t_{L}\right)=\sum_{n=0}^{\infty} F_{T_{f}}\left(t_{L} \mid N\left(t_{L}\right)=n\right)
$$

With the load occurrences being sufficiently rare events, only a few terms need to be considered in the series (eq 6.44).

\footnotetext{
${ }^{12}$ In CDM, failure is defined as the formation of a macro- crack.
} 


\subsubsection{Numerical Example}

A schematic representation of creep damage accumulation in a corroding steel ringstiffened shell subjected to internal pressurization is shown by the sample function in Figure 6.8. A pressurization event occurs at each discontinuity of the sample path ${ }^{13}$. The instantaneous jumps underline the fact that the duration of the elevated temperature is very small compared to the scale of reliability computations. The acceleration in damage growth later in the life of the structure is partly due to the increasing corrosion loss which amplifies the shell membrane stress.

Table 6.4 and Figure 6.9 show the reliability due to creep damage accumulation in the steel shell as a function of time. Creep parameters for A36 steel (Harmathy, 1967) have been used for the purpose of illustration ${ }^{14}$. The noise parameter $\sqrt{ } c_{4} / c_{3}=20 k s i \sqrt{ } h r$ has been adapted from the examples presented in section 5.5 .2 and the true failure stress has been assumed constant at $552 \mathrm{MPa}(80 \mathrm{ksi})$. The statistics of the pressure, temperature and their duration are presented in Table 6.1. A correlation coefficient of 0.60 between $\theta_{a}$ and $P_{a}$ has been assumed ${ }^{15}$. The containment is assumed to start service in an undamaged state, and the critical damage is assumed to be normally distributed with mean 0.5 (consistent with the results predicted in Tables 4.5 and 4.6) and COV $20 \%$. The conditional failure probabilities (Table 6.4) increase with the number of events; however the probabilities of 2 or more occurrences rapidly decrease, and not more than three terms need to be considered in the expansion (eq 6.44). The cumulative failure probability (CFP) due to creep damage accumulation (Fig 6.9) is orders of magnitude lower than that due to the deformation (maximum strain) limit state associated with internal pressurization (Fig. 6.4a) under similar conditions. Thus, elevated temperature creep of the shell clearly is unimportant as a limit state.

\footnotetext{
${ }^{13}$ The rate of occurrence of events has been greatly magnified in this figure to illustrate the concept

${ }^{14}$ Harmathy provides a functional relation between temperature and the rate, $A$, and treats $m=4.7$ for all temperatures. The range of temperatures in the present analysis is lower than the range where Harmathy's relations are valid, but they are nevertheless adapted here.

${ }^{15} \mathrm{~A}$ correlation coefficient of 0.62 between the the bivariate normal deviates $z_{1}$ and $z_{2}$ produced the correlation coefficient of 0.60 between $\theta_{a}=F_{\theta}^{-1}\left(\Phi\left(z_{1}\right)\right)$ and $P_{a}=F_{P}^{-1}\left(\Phi\left(z_{2}\right)\right)$.
} 


\begin{tabular}{|c|c|c|}
\hline Variable & Nominal value & Statistical properties \\
\hline \hline radius $r$ & $16.8 \mathrm{~m}(55 \mathrm{ft})$ & deterministic \\
\hline original thickness, $h_{0}$ & $34.9 \mathrm{~mm}(1.375 \mathrm{in})$ & deterministic \\
\hline Stringer area, $A_{1}$ & $15500 \mathrm{~mm}^{2}\left(24.0 \mathrm{in}^{2}\right)$ & deterministic \\
\hline Stringer spacing, $s_{1}$ & $3 \mathrm{~m}(10 \mathrm{ft})$ & deterministic \\
\hline $\begin{array}{c}\text { Accidental pressure, } P_{a} \\
\text { (normalized), } Y=P_{a} / P_{a n}\end{array}$ & $276 \mathrm{kPa}(40 \mathrm{psig})$ & Type I max $(0.8,20 \%)$ \\
\hline $\begin{array}{c}\text { Yield stress, } F_{y} \\
\text { (normalized), } X=F_{y} / F_{y n}\end{array}$ & $262 \mathrm{MPa}(38 \mathrm{ksi})$ & Lognormal $(1.10,7 \%)$ \\
\hline Tensile strength, $F_{u}$ & $483 \mathrm{MPa}(70 \mathrm{ksi})$ & \\
\hline Significant duration, $\Delta t$ & & Lognormal $(1000 \mathrm{~s}, 30 \%)$ \\
\hline Accidental temperature, $\theta_{a}$ & $199^{\circ} \mathrm{C}\left(390^{\circ} \mathrm{F}\right)$ & Type I max $\left(350^{\circ} \mathrm{F}, 20 \%\right)$ \\
\hline & & A. \\
\hline
\end{tabular}

Table 6.1: Nominal (uncorroded) dimensions, SA 516/70 material properties, and load and resistance statistics for a typical NPP steel containment. The two statistical parameters in the parentheses are mean and COV. 


\begin{tabular}{|c|c||c|c|c|}
\hline $\begin{array}{c}\text { Corrosion } \\
\text { scenario }\end{array}$ & $\begin{array}{c}\text { Restriction } \\
\text { imposed }\end{array}$ & $\begin{array}{c}\text { rate }\left(\mu m / y r^{M}\right) \\
M\end{array}$ & $\begin{array}{c}\text { exponent } \\
M\end{array}$ & $\begin{array}{c}\text { Initiation time } \\
T_{I}(y r)\end{array}$ \\
\hline \hline "Severe" & none & $\mathrm{LN}(600,30 \%)$ & $\mathrm{N}(0.7,20 \%)$ & $\mathrm{LN}(10,30 \%)$ \\
\cline { 2 - 5 } & $C$ deterministic & 600 & $\mathrm{~N}(0.7,20 \%)$ & $\mathrm{LN}(10,30 \%)$ \\
\cline { 2 - 5 } & $M$ deterministic & $\mathrm{LN}(600,30 \%)$ & 0.7 & $\mathrm{LN}(10,30 \%)$ \\
\cline { 2 - 5 } & $T_{I}$ deterministic & $\mathrm{LN}(600,30 \%)$ & $\mathrm{N}(0.7,20 \%)$ & 10 \\
\cline { 2 - 5 } & Ignore $T_{I}$ & $\mathrm{LN}(600,30 \%)$ & $\mathrm{N}(0.7,20 \%)$ & 0 \\
\hline \hline "Moderate" & none & $\mathrm{LN}(230,30 \%)$ & $\mathrm{N}(0.7,20 \%)$ & $\mathrm{LN}(10,30 \%)$ \\
\hline \hline "Zero" & - & 0 & & \\
\hline \hline
\end{tabular}

Table 6.2: Different cases of uniform corrosion of the NPP steel containment. The statistical parameters enclosed in parentheses are mean and COV.

\begin{tabular}{|c||c|c|c|}
\hline \multicolumn{1}{|c||}{} & \multicolumn{3}{c|}{ Shape parameter, $\alpha$} \\
\cline { 2 - 4 } Mean failure time, $\mu$ & $\begin{array}{c}1.5 \\
(\mathrm{COV}=67.9 \%)\end{array}$ & $\begin{array}{c}2 \\
(\mathrm{COV}=52.3 \%)\end{array}$ & $\begin{array}{c}3 \\
(\mathrm{COV}=36.3 \%)\end{array}$ \\
\hline $120 \mathrm{yr}\left(=2 t_{L}\right)$ & $26.2 \%$ & $17.8 \%$ & $8.5 \%$ \\
\hline $300 \mathrm{yr}\left(=5 t_{L}\right)$ & $7.4 \%$ & $3.1 \%$ & $0.6 \%$ \\
\hline $600 \mathrm{yr}\left(=10 t_{L}\right)$ & $2.7 \%$ & $0.8 \%$ & $0.07 \%$ \\
\hline$\infty$ (no aging) & 0 & 0 & 0 \\
\hline \hline
\end{tabular}

Table 6.3: Different cases of aging of safety-related equipment (assumed Weibull distributed) and corresponding failure probabilities at $60 \mathrm{yrs}$ 


\begin{tabular}{|c|c|c|c|}
\hline$t_{L}(\mathrm{yr})$ & $\bar{n}$ & $\mathrm{P}\left[N\left(t_{L}\right)=n\right]$ & $F_{T_{f}}\left[t_{L} \mid N\left(t_{L}\right)=n\right]$ \\
\hline 10 & $\begin{array}{l}1 \\
2 \\
3\end{array}$ & $\begin{array}{l}0.12167 \times 10^{-2} \\
0.74106 \times 10^{-6} \\
0.30091 \times 10^{-9}\end{array}$ & $\begin{array}{l}0.49171 \times 10^{-8} \\
0.84052 \times 10^{-6} \\
0.30605 \times 10^{-5}\end{array}$ \\
\hline 20 & $\begin{array}{l}1 \\
2 \\
3\end{array}$ & $\begin{array}{l}0.28644 \times 10^{-2} \\
0.41143 \times 10^{-5} \\
0.39396 \times 10^{-8}\end{array}$ & $\begin{array}{l}0.49171 \times 10^{-8} \\
0.84062 \times 10^{-6} \\
0.31556 \times 10^{-5}\end{array}$ \\
\hline 30 & $\begin{array}{l}1 \\
2 \\
3\end{array}$ & $\begin{array}{l}0.49389 \times 10^{-2} \\
0.12257 \times 10^{-4} \\
0.20279 \times 10^{-7}\end{array}$ & $\begin{array}{l}0.11489 \times 10^{-5} \\
0.10696 \times 10^{-5} \\
0.60714 \times 10^{-5}\end{array}$ \\
\hline 40 & $\begin{array}{l}1 \\
2 \\
3\end{array}$ & $\begin{array}{l}0.74348 \times 10^{-2} \\
0.27846 \times 10^{-4} \\
0.69527 \times 10^{-7}\end{array}$ & $\begin{array}{l}0.11908 \times 10^{-5} \\
0.11298 \times 10^{-5} \\
0.62590 \times 10^{-5}\end{array}$ \\
\hline 50 & $\begin{array}{l}1 \\
2 \\
3\end{array}$ & $\begin{array}{l}0.10345 \times 10^{-1} \\
0.54076 \times 10^{-4} \\
0.18844 \times 10^{-6}\end{array}$ & $\begin{array}{l}0.12273 \times 10^{-5} \\
0.12036 \times 10^{-5} \\
0.18430 \times 10^{-4}\end{array}$ \\
\hline 60 & $\begin{array}{l}1 \\
2 \\
3\end{array}$ & $\begin{array}{l}0.13663 \times 10^{-1} \\
0.94646 \times 10^{-4} \\
0.43707 \times 10^{-6}\end{array}$ & $\begin{array}{l}0.14760 \times 10^{-4} \\
0.28301 \times 10^{-4} \\
0.44587 \times 10^{-4}\end{array}$ \\
\hline
\end{tabular}

Table 6.4: Conditional failure probabilities due to creep damage growth in NPP containment shell 

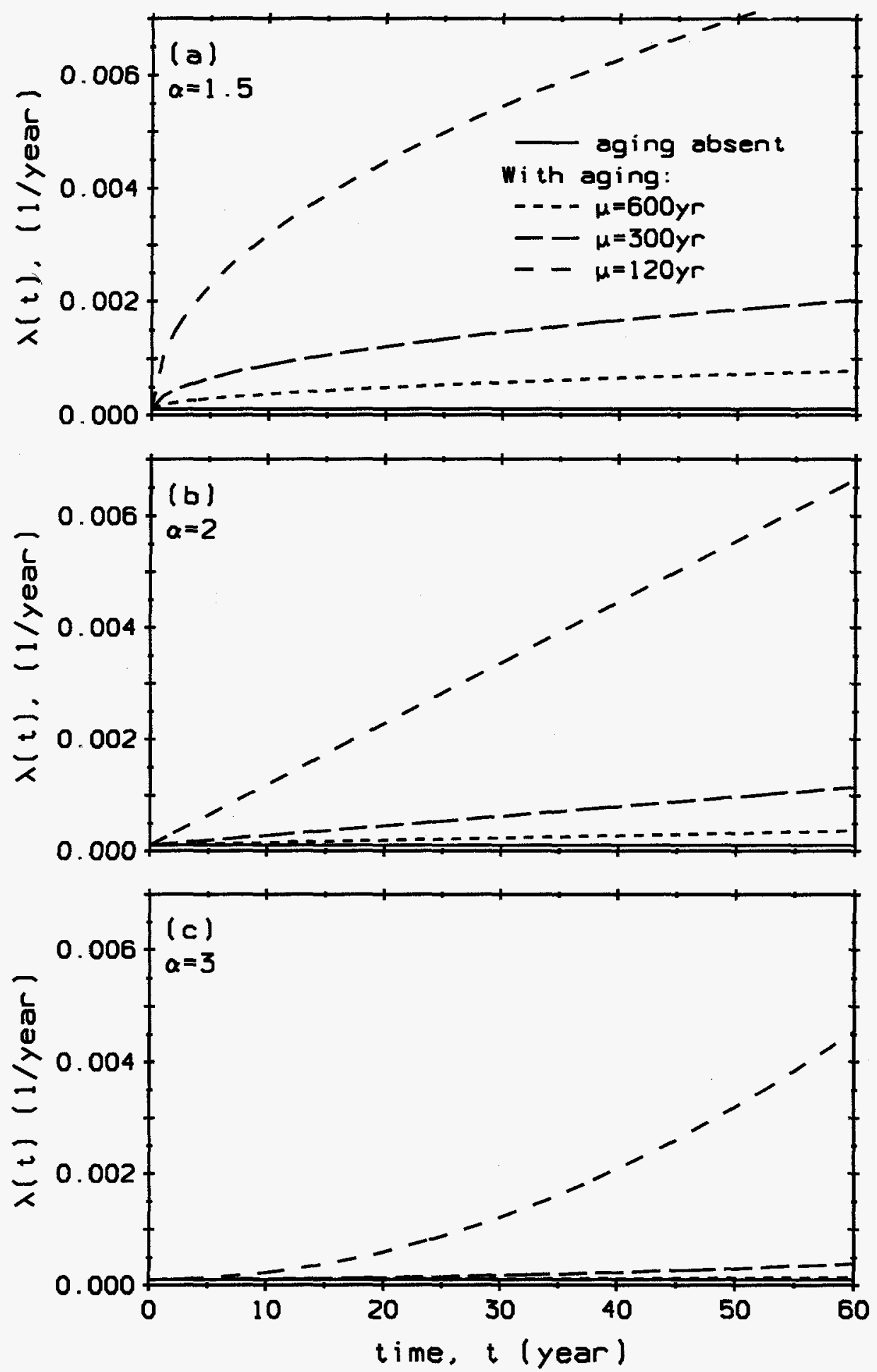

Figure 6.1: Poisson arrival rates of accidental pressures with $\lambda_{0}=0.0001 / y r$ (a) $\alpha=1.5$ (b) $\alpha=2$ (c) $\alpha=3$ 

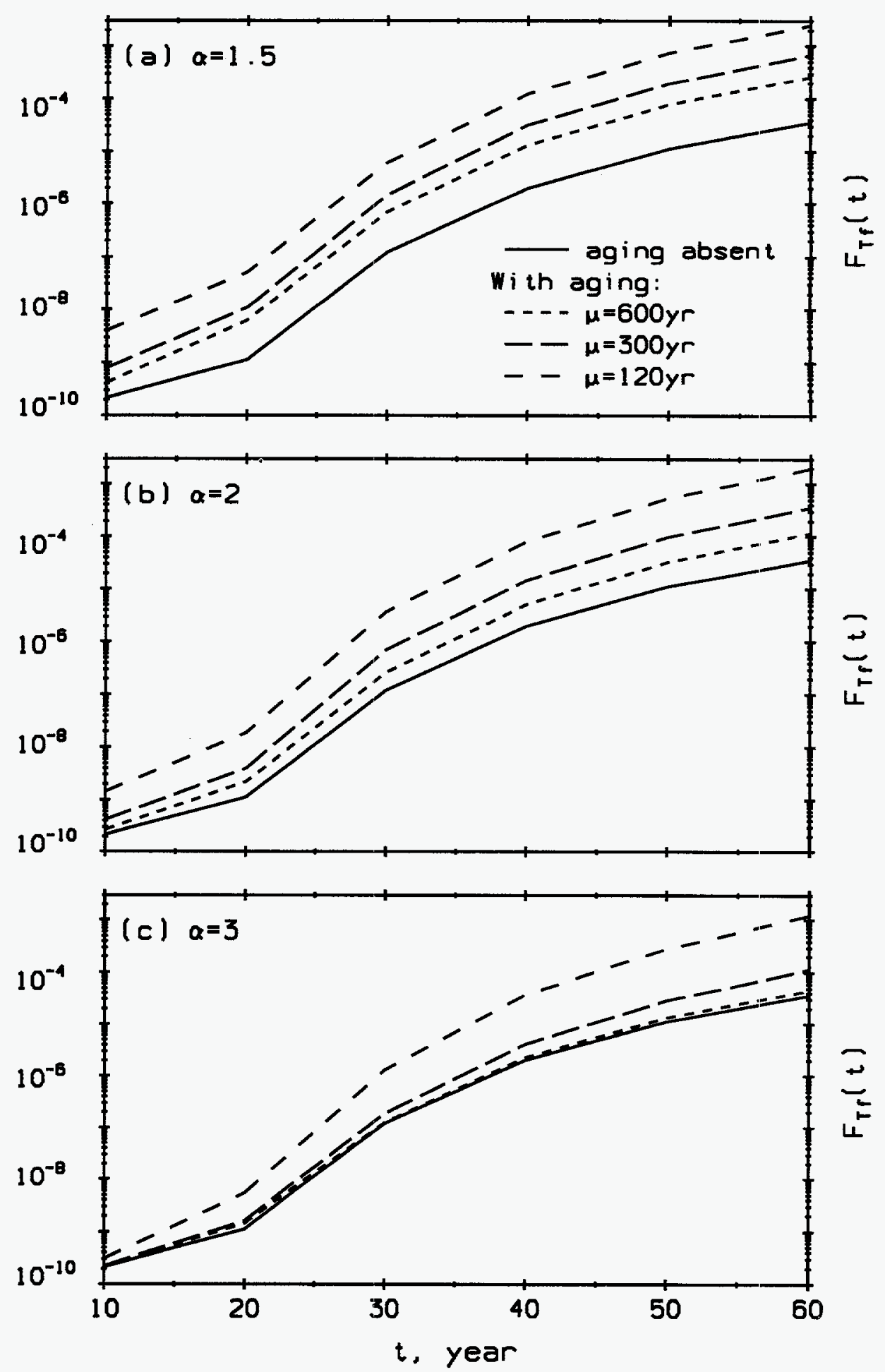

Е

Figure 6.2: CFP of ring-stiffened NPP steel containment subjected to internal pressurization, severe corrosion and $\lambda_{0}=0.0001 / y r$ (a) $\alpha=1.5$ (b) $\alpha=2$ (c) $\alpha=3$ 


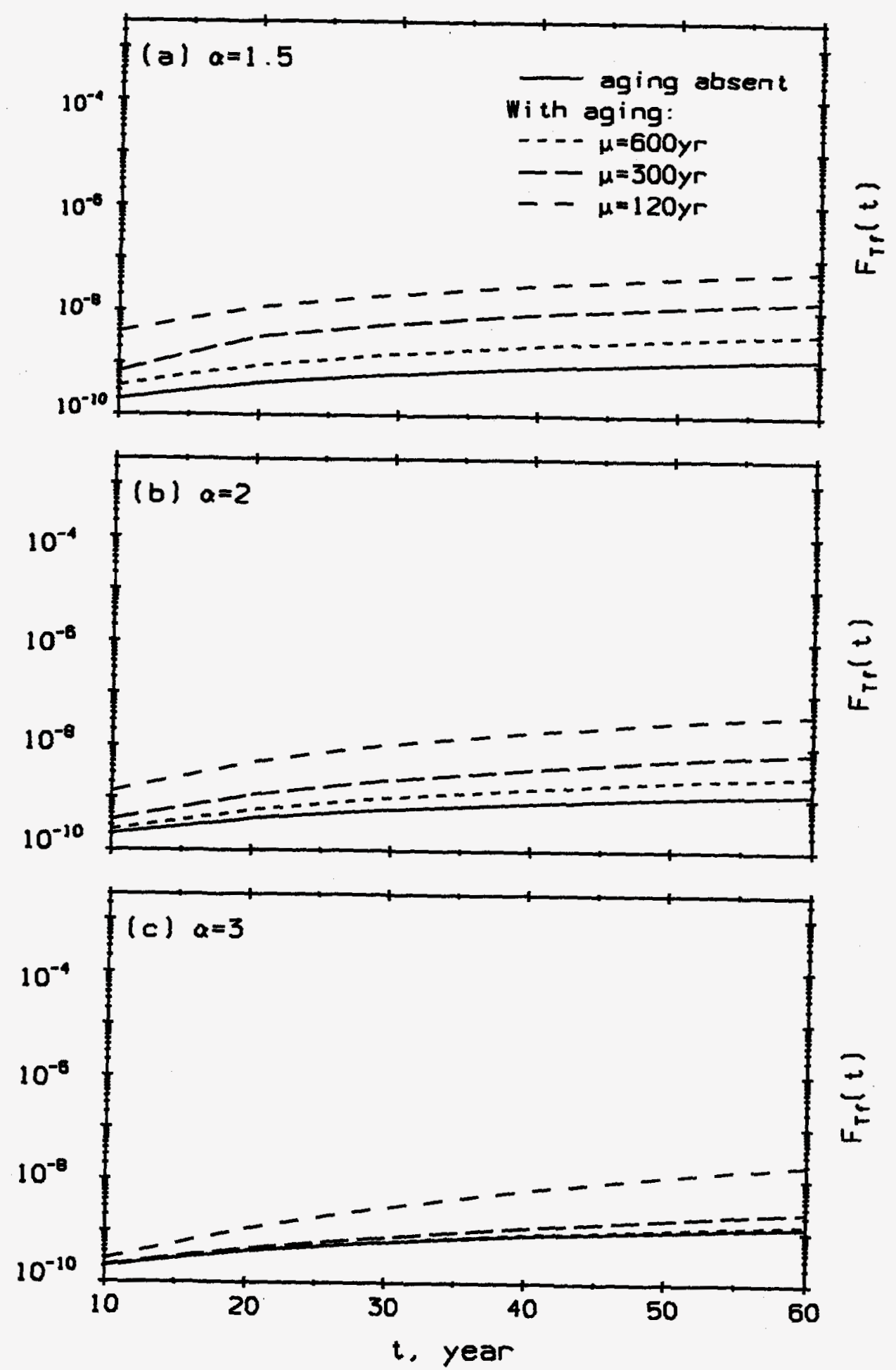

Figure 6.3: CFP of ring-stiffened NPP steel containment subject to internal pressurization in absence of corrosion with $\lambda_{0}=0.0001 / y r$ (a) $\alpha=1.5$ (b) $\alpha=2$ (c) $\alpha=3$ 

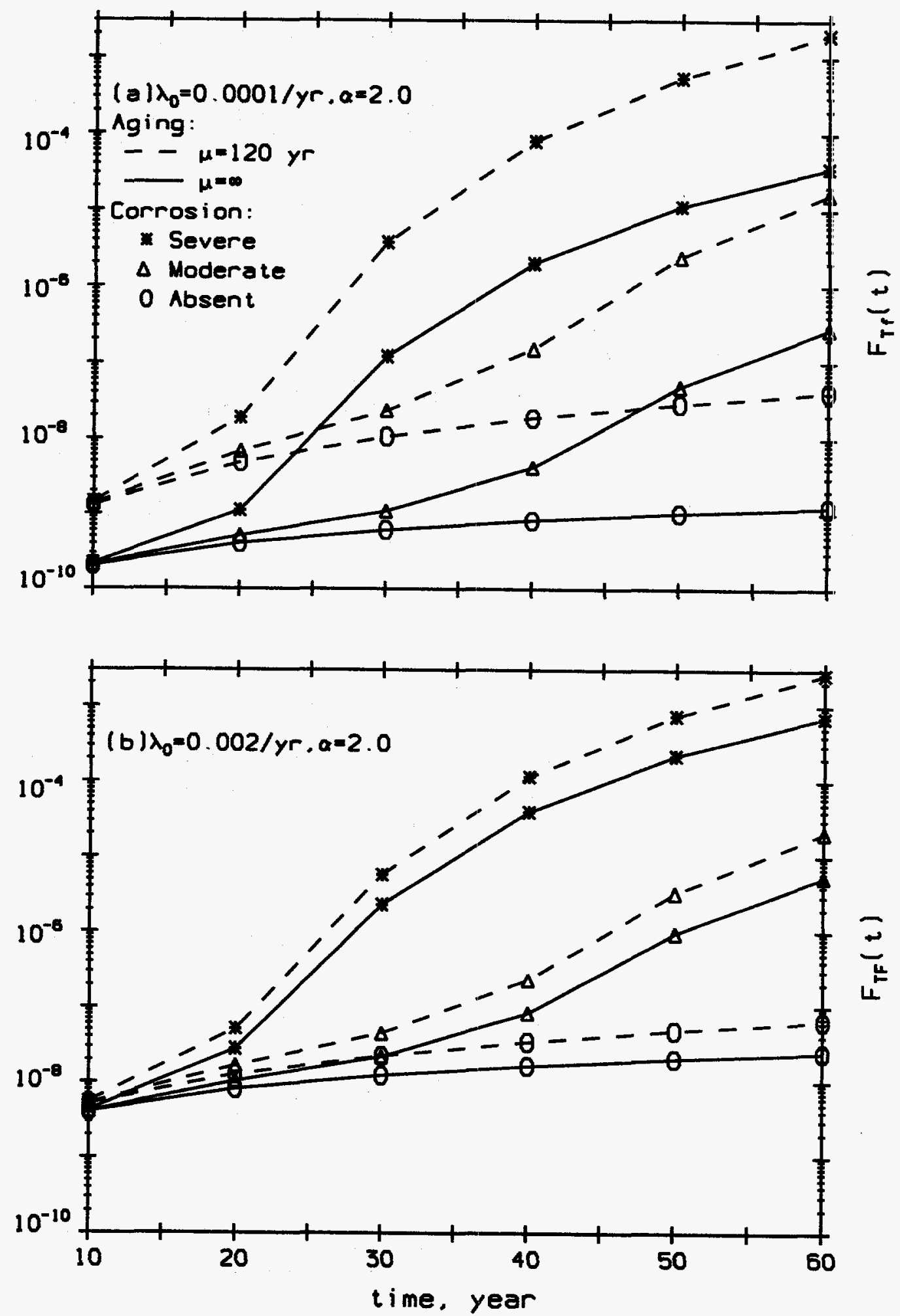

Figure 6.4: Effects of corrosion rate on reliability with and without aging (a) $\lambda_{0}=$ $0.0001 / y r$ (b) $\lambda_{0}=0.002 / y r$. 

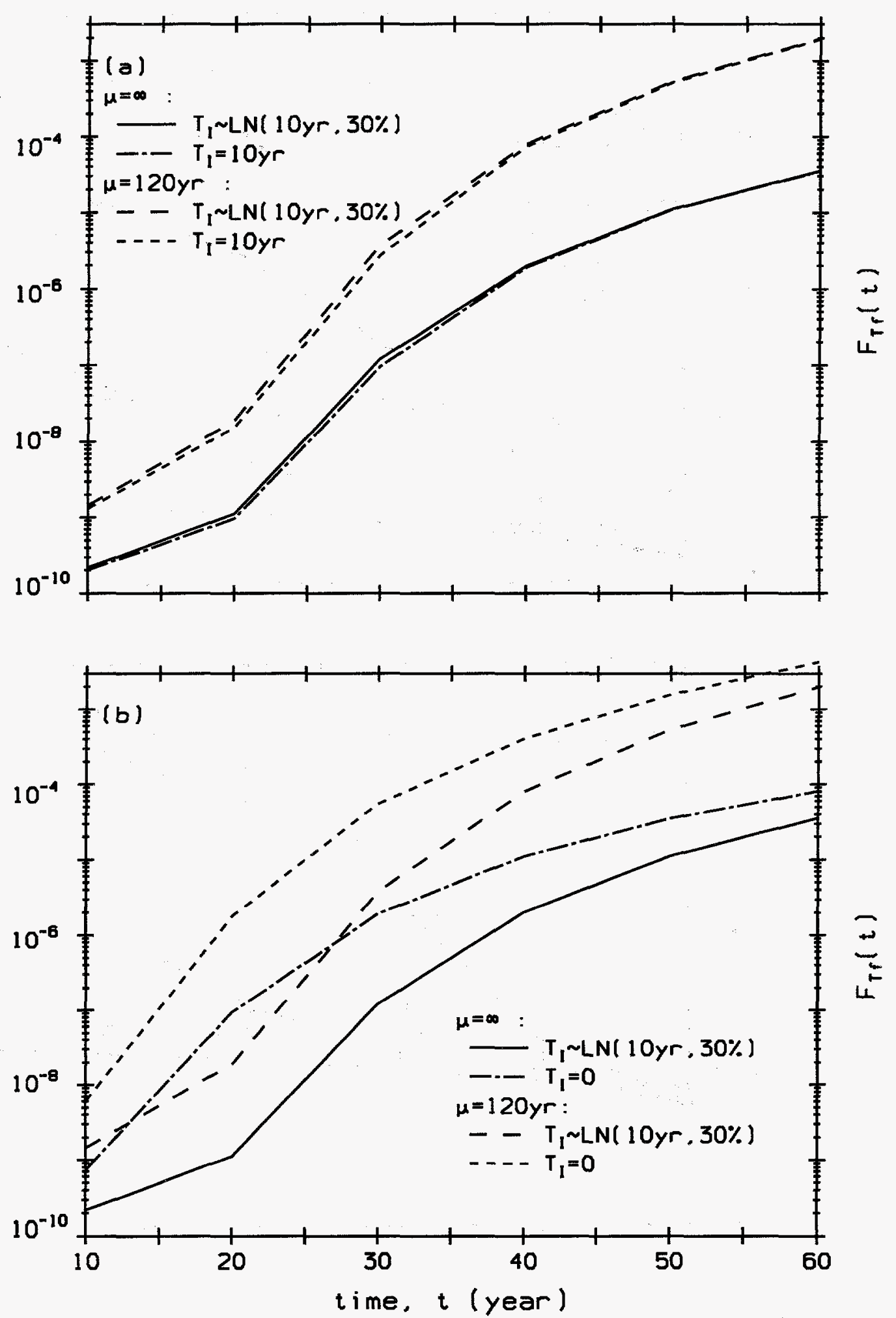

Figure 6.5: Effect of (a) setting initiation time equal to its mean and (b) ignoring initiation time (with $\lambda_{0}=0.0001 / y r, \alpha=2$ ) 


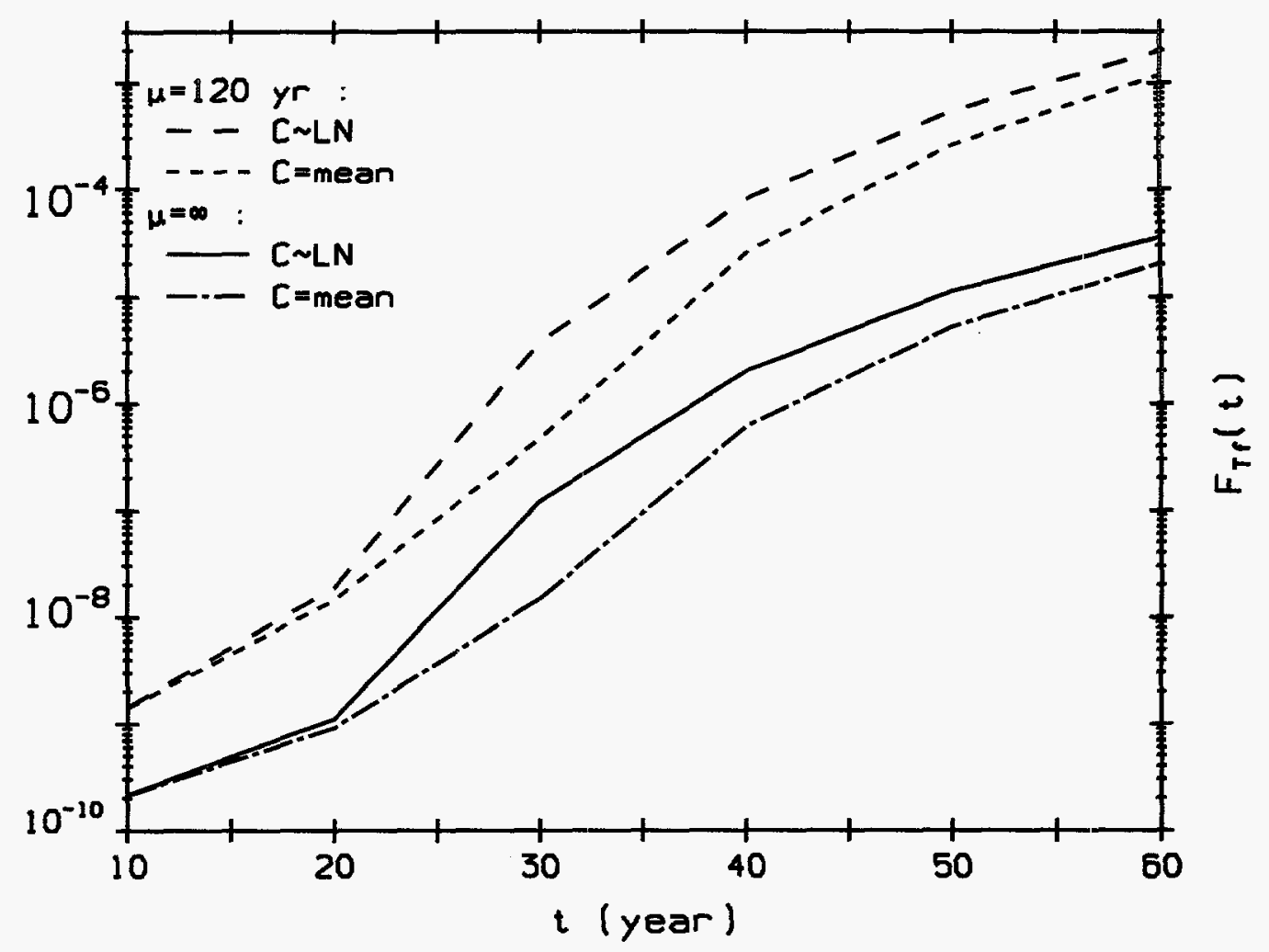

Figure 6.6: Effect of setting corrosion rate equal to its mean under severe corrosion $\left(\lambda_{0}=0.0001 / y r, \alpha=2\right)$ 


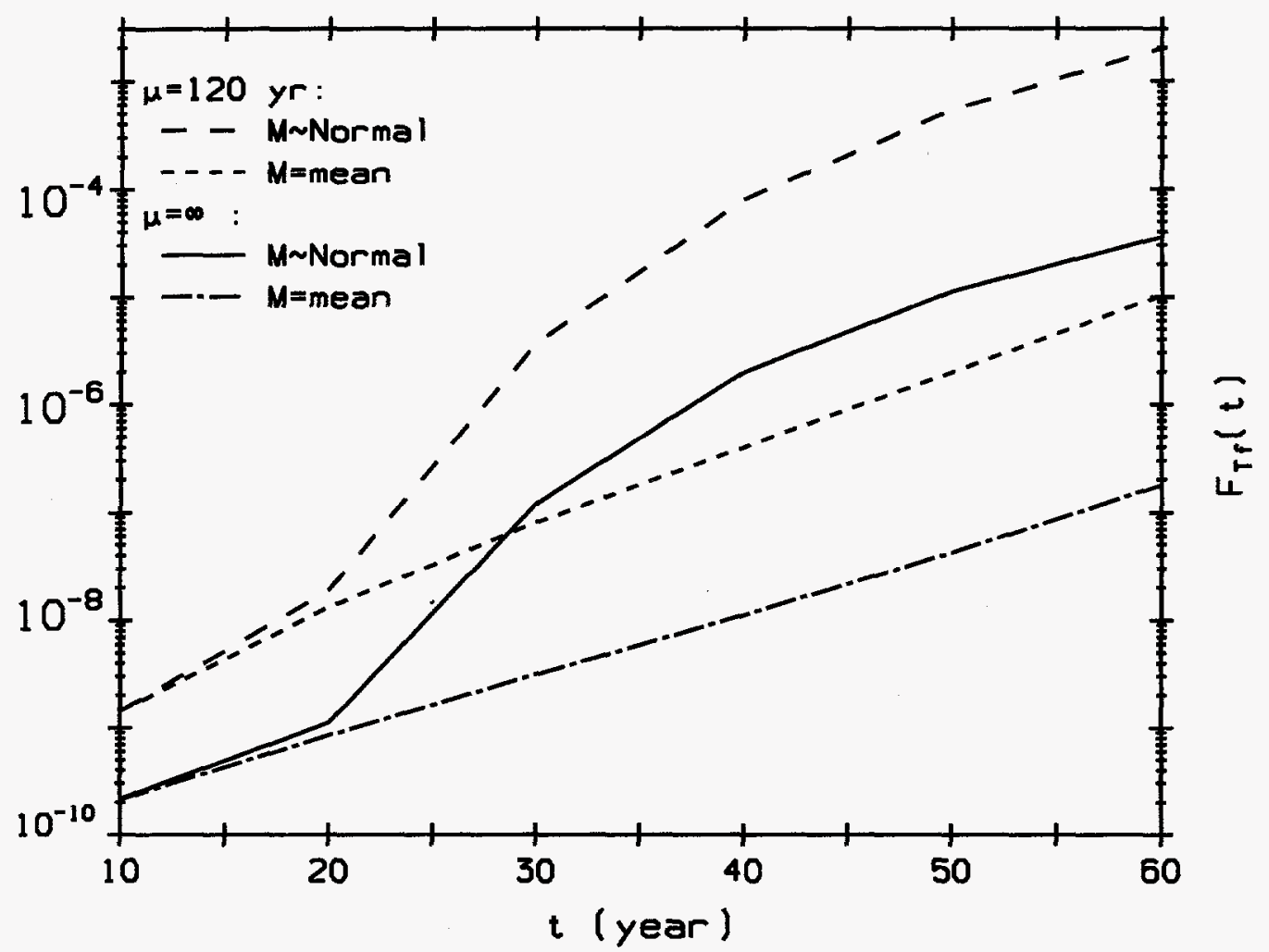

Figure 6.7: Effect of setting corrosion exponent equal to its mean under severe corrosion $\left(\lambda_{0}=0.0001 / y r, \alpha=2\right)$ 


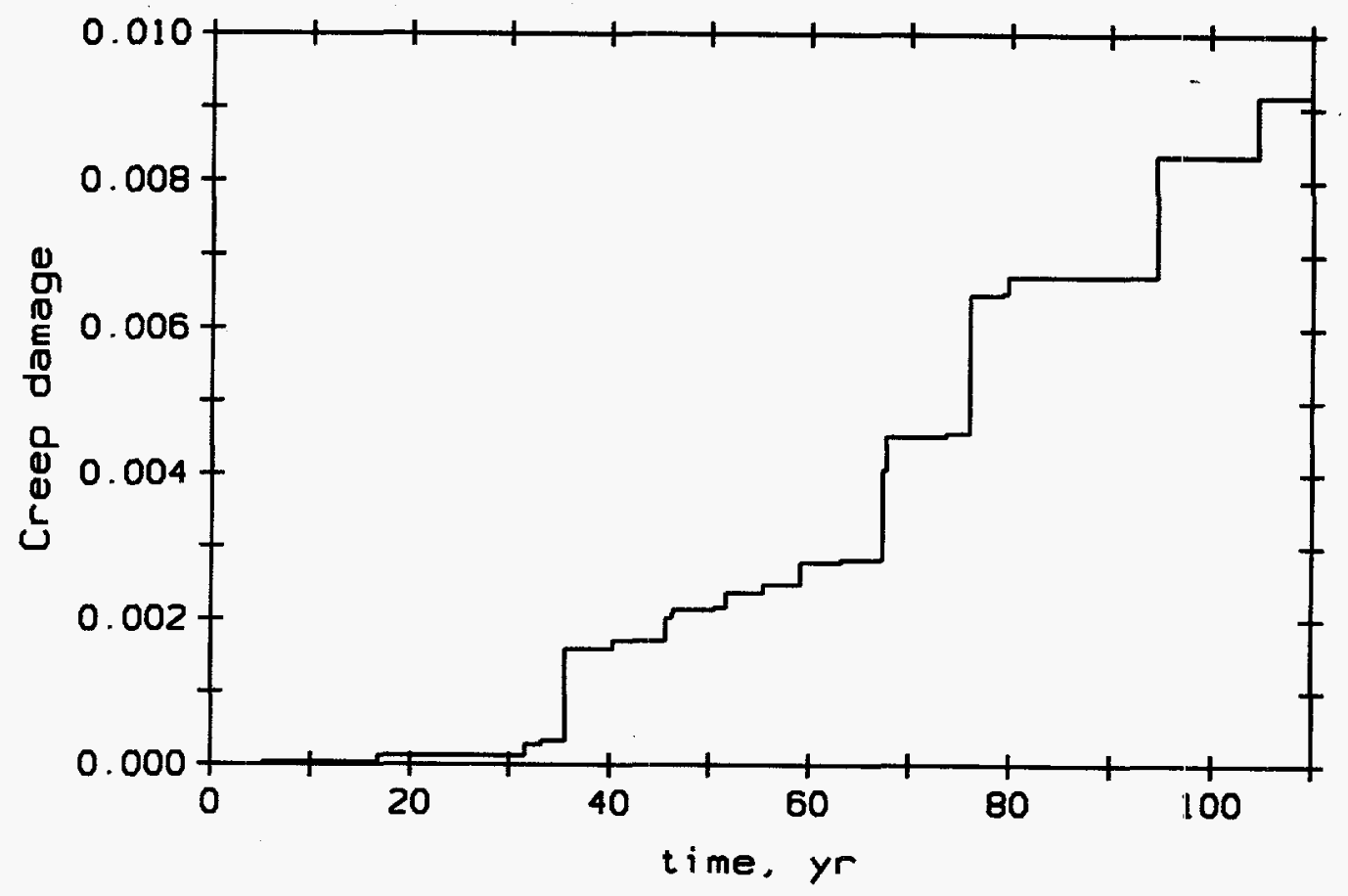

Figure 6.8: Sample path of random creep damage growth in steel containment shell 


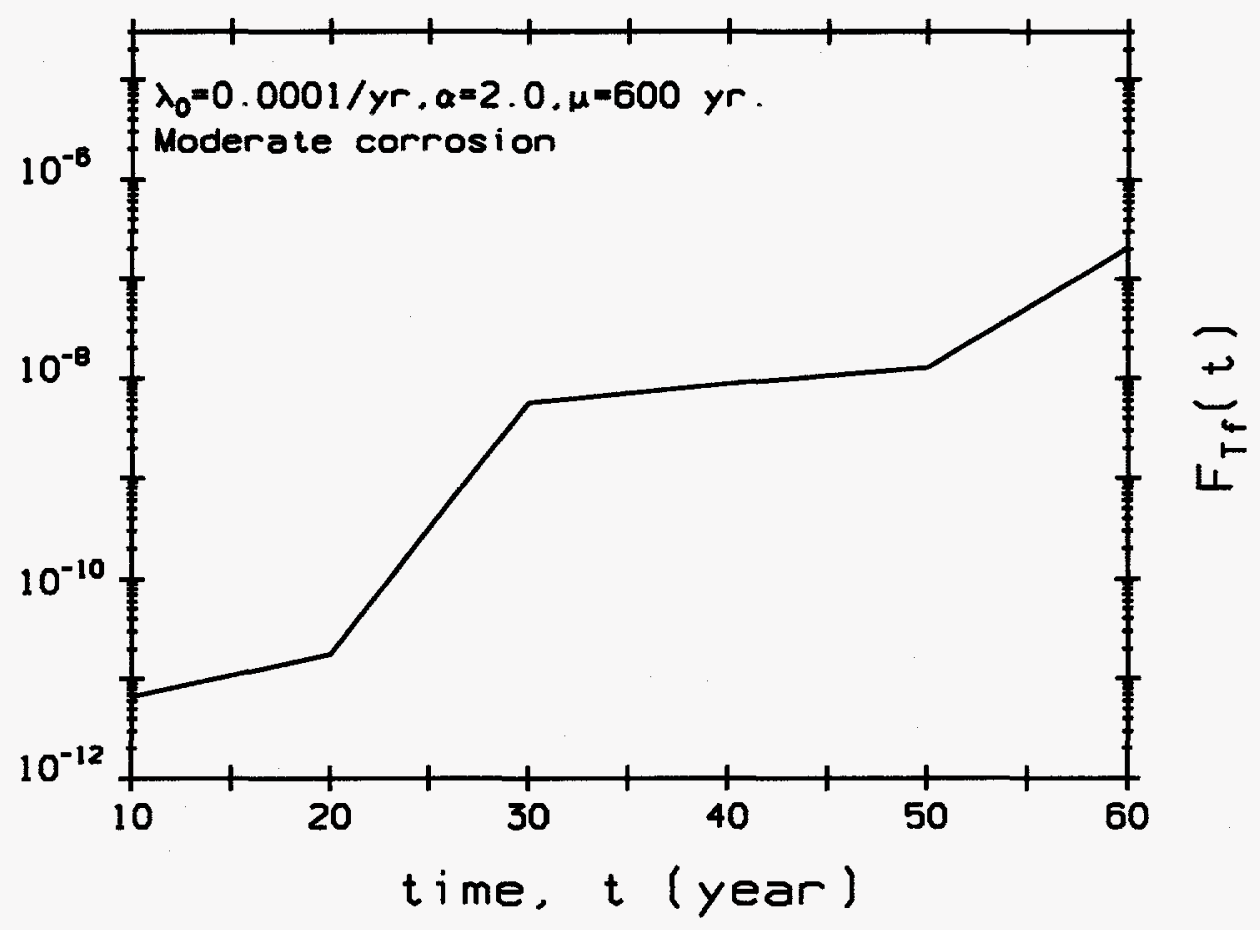

Figure 6.9: Cumulative failure probability in creep damage accumulation 



\section{Chapter 7}

\section{Conclusions and Recommendations}

\subsection{Summary of Research and Findings}

The research presented in this report had two principal motivations. The first was to develop rational damage growth laws for deteriorating structures with as little empiricism as possible. The second was to model the randomness in damage growth from a more fundamental perspective than to simply "randomize" the deterministic equations.

Most of the existing models of structural deterioration were found to rely heavily on curve-fitting with little or no ties to the mechanics of the process. This turns out to be a serious deficiency when attempting to determine the remaining capacity of a damaged structure, since empirical methods usually are unable to provide estimates of residual strength and stiffness. Along with this shortcoming, most of the existing methods were also found to require a measurable flaw to be applicable, leaving the important area of pre-nucleation damage growth largely out of their purview. In contrast, the relatively new field of continuum damage mechanics (CDM) offers a definition of damage that can be directly related to the mechanical properties of the structure and, what is equally appealing, has a natural advantage in modeling pre-nucleation damage accumulation, owing to the way it defines failure.

Existing CDM-based models of structural deterioration, however, were found handicapped by the presence of undetermined material constants. Moreover, the need to postulate kinetic or dissipation potential functions had a certain amount of arbitrariness about it. No one seemed to have exploited the powerful potential of CDM to model random damage growth, by linking the microstructural fluctuations in energy and material properties (spatial and temporal) to the stochastic nature of damage observed in the macro-scale. 
Physically, damage growth is an irreversible process and the thermodynamic formulation of damage accumulation was already in place in the existing CDM-based approaches. The present research built up on those results and applied them to deformable bodies in which damage accumulation was proceeding close to equilibrium under isothermal conditions. The resulting damage growth equations were shown to depend on the evolution of the Helmholtz free energy of the deformable body. Since this work primarily concerned metals having structural applications, it was deemed adequate to consider damage as isotropic. This also avoided complicating the development with tensor notations and losing sight of the practical relevance of the damage concept. It also facilitated verification of the proposed model, as most of the published experimental data were on the scalar damage variable.

The damage accumulation model developed in this research and summarized above is general in its scope and can be used to express the effects of different processes using one unifying approach. In essence, it is based on a concept of damage that is linked directly to the state of the microstructure which, through a simple equivalence relation between a damaged and a comparable undamaged body (eg, the principle of strain equivalence), relates the structural response to the state of damage within the structure. The Helmholtz free energy, which is central to the proposed model, can be defined for any isothermal process to any desired degree of completeness and can be achieved without introducing unknown material parameters. The more complete the constitutive equations defining the free energy of the deformable body, the better is the performance of the proposed model. Finally, the definition of failure in CDM is not particular to any damage-causing process: Failure occurs when the microstructure anywhere within the continuum volume degenerates into a macroscopic flaw. The critical damage is thus not required to be identically equal to 1 .

The surface energy of formation of microscopic voids was derived under a set of simplifications which, among others, made the voids spherical, but nevertheless, allowed the surface energy to be explicitly dependent on the state of damage. The substitution of the local failure stress with the true fracture stress was found to produce good results.

\section{Ductile Deformation Damage}

The resulting equations for monotonic ductile damage growth were shown to have similar algebraic forms to those arising out of the existing dissipation potential approach. The improvement of course was the absence of undetermined parameters. The proposed model was found to be sensitive to the hardening modulus and the true failure stress. Varying the hardening exponent, however, was found to have a secondary effect on damage growth . Significant differences were found in the published experimental values of these parameters for the same nominal material grade. Varying the elasticity modulus had almost no effect on damage growth, and interestingly, little variability was observed in the numerical estimates of the elasticity modulus, unlike the other three parameters. 
Estimates of the threshold plastic strain of damage accumulation for all the materials considered were found to be infinitesimal compared to the corresponding fracture ductilities, and the threshold strain could be completely ignored insofar as predicting failure was concerned. The predictions of ductile damage growth were found to lie a little on the high side of the experimental results in all the cases considered. This may be due in part to the idealization of the voids as spheres, since spheres have the least surface area for a given volume.

\section{Creep Damage}

In creep damage, the principle of equivalence between a damaged and a comparable undamaged body was applied to the rate of strain, rather than to the strain itself. This led to a creep damage growth equation which, under steady state conditions, had an algebraic form identical to those arising out of the existing kinetic equation approach, with one important difference: the parameters of the proposed model were all determinate. Considering the steady-state equation alone was found to underpredict damage growth, since the steady-state pertained to the minimum rate of creep strain accumulation. Including the non-zero initial damage caused by the time-independent plastic deformation was felt necessary to correct the imbalance. The predicted creep damage growth was found to be highly nonlinear (accelerating) with time, and the failure time became increasingly insensitive to the critical damage as the latter's value approached unity.

\section{Fatigue Damage}

The concept that the critical damage corresponds to the formation of the first macrodefect was most convincingly demonstrated in the case of fatigue damage growth. The predicted number of cycles to failure was found to match the observed crack-initiation life very well in all the cases considered. A comment about the definition of fatigue crack initiation may be in order here: Since it is more difficult to identify and measure a threshold crack length, and since no consensus about the definition of initiation crack length exists, the definition of crack initiation may be shifted from a crack length-based approached to a stiffness-based approach, which may be more universally acceptable in the fatigue research community.

The fatigue damage model was based on the irreversibility of damage accumulation. The damage at the end of any given cycle was assumed to act as the initial value for the damage increment in the next cycle. The model was able to differentiate between straincontrolled and stress-controlled fatigue cycling, could predict the S-N curve corresponding to initiation, and could capture qualitatively the effects of load sequencing. 


\section{Random Damage Growth}

The treatment of random damage growth in this report took a more fundamental approach than to simply "randomize" the deterministic equations or its parameters. Using the knowledge from statistical thermodynamics that a system in macroscopic equilibrium undergoes continuous and rapid fluctuations in its thermodynamic properties at the micro-scale, the Helmholtz free energy was now considered a stochastic process with the deterministic counterpart as its mean function. Assuming certain properties for the fluctuations in the free energy, a stochastic differential equation of damage growth in the near-equilibrium deformable body was obtained, which was then applied to the specific cases of ductile deformation, creep and fatigue damages. Closed-form solutions of the SDEs were obtained in each case and the Ito and the Stratonovich approaches were compared. In the limiting case when noise in the process was absent, and the material properties and intial conditions were all precisely known, the stochastic equations were shown to reduce to their deterministic counterparts.

Numerical examples and comparisons with experimental data showed that estimates of the noise in the growth rate as well as knowledge of material variability were needed to completely characterize random damage growth. This observation was significant against: the backdrop of existing methods many of which consider randomness in one but not in the other.

The above stochastic models admit the possibility of negative damage growth. Even though such retardations in damage might actually take place locally and instantaneously, overall decrease in damage is a physical impossibility. It was shown through the numerical examples that the probability of such retardation occurring over a macroscopic time and space interval was vanishingly small. One important consequence of non-negative random damage growth is that a first passage problem can be reduced to a much simpler unconditional probability of a single event.

\section{Reliability of Steel Containment}

The damage growth models developed in this research were applied to investigate the time-dependent reliability of a ring-stiffened steel cylindrical shell typical of a containment in an NPP. The design basis accidents were modeled to have two independent sources: one was due to pure chance, and the other due to aging of equipment. General corrosion of the containment steel was found to affect significantly the deformation limit state due to internal pressurization. On the other hand, the probability of failure due to creep in a design basis accident was found to be orders of magnitude less than that due to excessive deformation. Increasing the rate of occurrence of design-basis events had an adverse impact on the reliability of the shell. A study to find out whether frequent testing of safety-related equipment does make it more likely to malfunction at a later time is recommended. 


\subsection{Suggestions for Future Work}

Experimental database: The verification of the models derived in this report was not easy nor complete, as sufficient experimental data were not available in many cases, especially for creep and fatigue. Damage growth data are required to verify and improve these models. Monotonic tension tests conducted at different temperatures are recommended which may help create a database of critical damage parameters.

Critical damage: Further experimental work is needed to determine to what extent the critical damage is a material constant, and what restrictions, if any, need to be imposed on the postulate.

Noise: The ratio of the standard deviation and the correlation length of the noise in the damage growth process is needed in the proposed model. This research could not provide analytical expressions of these two quantities and best estimates had to be obtained from the experimental scatter. While numerical estimates can be obtained through the experimental determination of the variance and covariance functions of damage, analytical expressions should be developed. The noise is expected to be strongly temperature-dependent.

Corrosion: Corrosion was treated empirically in this research. It is believed that corrosion can also be modeled using CDM based methods. Gibbs free energy seems to be a logical starting point for this.

Strain-rate effects: The strain-rate effects may be modeled by including the viscosity term in the free energy.

Non-equilibrium conditions: This research modeled damage growth proceeding slowly and close to equilibrium. It may be useful to investigate the more general case of damage accumulating away from equilibrium. Failure is said to occur in CDM when localization takes place. In this light, a growing crack may be an example of damage growth away from equilibrium, and CDM may be extended to model crack growth occurring in a continuum.

Structural control: The stiffness of a degrading structure may be continuously monitored by one of several available NDE methods, which may then be fed into the models derived here to obtain the reliability of the structure. This may be integrated with a structural control system to optimize the performance of the structure. 



\section{Appendix A}

\section{Crack-tip Plasticity and Propagation Life}

To accommodate for crack-tip plasticity (of length $r_{y}$ ), the effective crack length, $a_{e}$, is

$$
\begin{aligned}
a_{e} & =a+r_{y} \\
r_{y} & =\frac{1}{\pi} \frac{K^{2}}{\sigma_{y}^{2}} \\
K & =F\left(\frac{a_{e}}{b}\right) S \sqrt{\pi a_{e}}
\end{aligned}
$$

where $S$ is the far-field applied stress, $\sigma_{y}$ is the yield stress, and $b$ is the width of the specimen. The geometric correction factor is:

$$
\begin{aligned}
F(\alpha) & =0.265(1-\alpha)^{4}+\frac{0.857+0.265 \alpha}{(1-\alpha)^{1.5}} \text { for edge crack } \\
& =\sqrt{\frac{1}{\cos (\pi \alpha / 2)}} \text { for center crack }
\end{aligned}
$$

The following equation is solved iteratively to obtain the effective crack length:

$$
a_{e}-F^{2}\left(\frac{a_{e}}{b}\right) \frac{S^{2}}{\sigma_{y}^{2}} a_{e}-a=0
$$

where $a$ is the nominal crack length (or half-length in case of a center crack). The maximum stress intensity factor, $K_{\max }$ can be obtained by putting $S=S_{\max }$ in eq (A.3). For stable crack growth:

$$
K_{\max } \leq K_{\text {crit }}
$$

the critical stress intensity factor being the smaller of the two:

$$
K_{c r i t}=\min \left\{K_{c}, K_{T}\right\}
$$


where $K_{c}$ is the fracture toughness and $K_{T}=\sqrt{E \sigma_{y} \delta_{T}}$ is the stress intensity factor corresponding to the crack tip opening displacement $\delta_{T}$ which is equal to $0.04 \mathrm{~mm}$ for most steel (Barsom and Rolfe, 1987, p. 281). For a tensile minimum stress, $K_{\min }$ may be obtained similarly as above by putting $S=S_{\min }$ in eq (A.3), yielding the stress intensity factor range as $\Delta K=K_{\max }-K_{\min }$.

The stress intensity factor range, $\Delta K$, in case of compressive minimum stress is obtained in the following way: The plastic zone length in compression is

$$
r_{y}^{\prime}=\frac{1}{\pi}\left(\frac{\Delta K}{2 \sigma_{y}}\right)^{2}
$$

which can be likewise used to iteratively solve the effective crack- length, $\boldsymbol{a}_{e}^{\prime}$, in compression in order to obtain $\Delta K$. The Paris Law may now be integrated between the limits $a=a_{s}$ (eq. 2.15) and $a=a_{f}$ subject to condition (A.7) above.

The material properties used to compute $N_{P}$ in Fig 4.18 are: $K_{c}=130 \mathrm{MPa} \sqrt{ } \mathrm{m}$, $\Delta K_{t h}=10 M P a \sqrt{ } m, \Delta S_{e}=1083 M P a, \delta_{T}=0.04 m m, \sigma_{y}=1180 M P a, b=6.35 m m$, $C=5.4 \times 10^{-11} \mathrm{~mm} / \mathrm{cycle}$ and $m=3.24$ which are taken from Dowling (1993), Barsom and Rolfe (1987) and Topper and Morrow (1970). 


\section{Bibliography}

[1] Abo El Ata, M. M. and Finnie, I. (1972). "A study of creep damage rules", Journal of Basic Engineering, 94:533-543.

[2] Agbabian, M.S., Masri, S.F., Miller, R.K., and Caughey, T.K. (1991). "System identification approach to detection of structural changes", Journal of Engineering Mechanics, 117(2):370-90.

[3] ASME (1986). ASME Boiler and Pressure Vessel Code, ASME.

[4] Barsom, J.M. and Rolfe, S.T. (1987). Fracture and Fatigue Control in Structures. Prentice-Hall.

[5] Berger, D.M. (1983). "Fundamentals and prevention of metallic corrosion", Corrosion and Corrosion Protection Handbook, ed: Schweitzer, P.A., Marcel Dekker.

[6] Bogdanoff, J.L. and Kozin, F. (1985). Probabilistic Models of Cumulative Damage, John Wiley and Sons.

[7] Boller, C. and Seeger, T. (1987). Materials Data for Cyclic Loading, Elsevier.

[8] Boyer, H. E. (1986). editor, Atlas of Fatigue Curves, ASM, Metals Park, OH.

[9] Boyer, H. E. (1987). editor, Atlas of Stress-Strain Curves, ASM International, Metals Park, OH.

[10] Boyer, H. E. (1988). editor, Atlas of Creep and Stress-Rupture Curves, ASM International, Metals Park, OH.

[11] Breysse, D. (1990). "Probabilistic formulation of damage-evolution law of cementitious composites." Journal of Engineering Mechanics, ASCE,116(7):1489-1510.

[12] Brockenbrough, R. L. and Johnston, B. G. (1968). USS Steel Design Manual, United States Steel Corpn., Pittsburgh, PA.

[13] Brockenbrough, R. L. and Merritt, F. S. (1994). Structural Steel Designer's Handbook, 2nd edition, McGraw-Hill.

[14] Callen, H. B. (1988). Thermodynamics and an Introduction to Thermostatics, John Wiley and Sons.

[15] Carmeliet, J. and Hens, H. (1994). "Probabilistic nonlocal damage model for continua with random field properties",Journal of Engineering Mechanics, ASCE, $120(10): 2013-27$.

[16] Chaboche, J.L. (1988) "Continuum damage mechanics - I and II", Journal of Applied Mechanics, 55:59-72. 
[17] Cherry, J. L. (1995). "Interim report on capacity of degraded containments", JCN no J6042, June, 1995, Sandia National Laboratories, NM.

[18] Cherry, J. L. (1996). "Analyses of Containment Structures with Corrosion Damage", Proc. Water Reactor Safety Information Meeting, Rockville, MD, October, 1996.

[19] Chopra, O. K. (1996). (of Argonne National Laboratory, Argonne, IL 60439, USA) Personal communication to Prof. B. R. Ellingwood of Johns Hopkins University, Baltimore, MD 21218 USA, regarding references [64, 65].

[20] Chow, C. L. and Wang, J. (1987). "An anisotropic theory of elasticity for continu um damage mechanics", International Journal of Fracture, 33:3-16.

[21] Chow, C.L. and Wei, Y. (1991). "A model of continuum damage mechanics for fatigue failure", International Journal of Fracture, 50(4):301-316.

[22] Clifton, J.R. and Knab, L.I. (1989). Service Life of Concrete. NUREG/CR-5466, US Nuclear Regulatory Commission, Washington, DC.

[23] Collins, J. A. (1981). Failure of Materials in Mechanical Design, John Wiley and Sons, New York.

[24] Committee on Fatigue and Fracture Reliability of the Committee on Structural Safety and Reliability of the Structural Division (1982a-d). "Fatigue reliability, I IV.", Journal of the Structural Division, ASCE, 108(ST1).

[25] Craig, B. D. (1989). ed Handbook of Corrosion Data, ASM International, Metals Park, $\mathrm{OH}$.

[26] Davis, J. R. (1994). Stainless Steels, ASM International, Materials Park, OH.

[27] deGroot, S. R. and Mazur, P. (1962). Non-Equilibrium Thermodynamics, NorthHolland, Amsterdam.

[28] deVree, J. H. P., Brekelmans, W. A. M., van Gils, M. A. J. (1995) "Comparison of nonlocal approaches in continuum damage mechanics", Computers and Structures, $55(4): 581-588$.

[29] Dhar, S., Sethuraman, R. and Dixit, P. M. (1996). "A continuum damage mechanics model for void growth and micro crack initiation", Engineering Fracture Mechanics, $53(6): 917-928$.

[30] Diao, X. (1995). "A statistical equation of damage evolution", Engineering Fracture Mechanics,52(1):33-42.

[31] DiPasquale, E. and Cakmak, A. S. (1989). "On the relation between local and global damage indices", Technical report,NCEER-89-0034, August, 1989.

[32] Ditlevsen, O. (1986). "Random fatigue crack growth-a first passage problem", Engineering Fracture Mechanics, 23(2):467-477.

[33] Dolinski, K. (1992). "Stochastic modeling and statistical verification of crack growth under constant amplitude loading". Engineering Fracture Mechanics, 43(2):195-216. 
[34] Dowling, N.E. (1993). Mechanical Behavior of Materials, Prentice Hall, 1993.

[35] Ellingwood, B. R. (1983). Probability Based Safety Checking of Nuclear Plant Structures, Report NUREG/CR-3628, US Nuclear Regulatory Commission, Washington, DC. 1983.

[36] Ellingwood, B. R. and Mori, Y. (1992). Condition Assessment and ReliabilityBased Life Prediction of Concrete Structures in Nuclear Plants, Report ORNL/NRC/LTR-92/4, US Nuclear Regulatory Commission, Washington, DC. 1992

[37] Ellingwood, B.R. and Mori, Y. (1993). "Probabilistic methods for condition assessment and life prediction of concrete structures in nuclear power plants", Nuclear Engineering and Design, 142:155-166.

[38] Ellingwood, B. R., Bhattacharya, B. and Zheng, R-H. (1996). Reliability- based Condition Assessment of Steel Containments and Liners, Report NUREG/CR 5442, US Nuclear Regulatory Commission, Washington, DC. 1996

[39] Endo, T. and Morrow, J. (1969). "Cyclic stress-strain and fatigue behavior of representative aircraft metals", Journal of Materials, JMLSA, 4(1):159-175.

[40] Fang, D. and Berkovits, A. (1995). "Fatigue design model based on damage mechanics revealed by acoustic emission measurements", Journal of Engineering Materials Technology, 117(2):200-208.

[41] Fields, B. A. and Fields, R. J. (1989). "Elevated temperature deformation of structural steel", em National Institute of Standards and Technology, Metallurgy Division, Gaithersburg, MD, NISTIR 88-3899.

[42] Gardiner, C. W. (1985). Handbook of Stochastic Methods for Physics, Chemistry and Natural Sciences, Springer-Verlag.

[43] Garofalo, R. W, et al (1961). "Creep and creep-rupture relationships in an austenitic stainless steel", Transactions of the Metallurgical Society of AIME, 221:310-319.

[44] Greimann, L. G. et al (1982a). Reliability Analysis of Steel Containment Strength, Report NUREG/CR-2442, US Nuclear Regulatory Commission, Washington, DC. June 1982.

[45] Greimann, L. G. et al (1982b). Reliability Analysis of Containment Strength, Report NUREG/CR-1891, US Nuclear Regulatory Commission, Washington, DC. August 1982.

[46] Handbook (1990) Metals Handbook, 10th ed., ASM International, Metals Park, $\mathrm{OH}$.

[47] Hansen, N.R. and Schreyer, H.L. (1994). "A thermodynamically consistent framework for theories of elastoplasticity coupled with damage", International Journal of Solids and Structures, 31(3):359-389.

[48] Harlow, D.G. and Delph, T. J. (1995). "A computational probabilistic model for creep-damaging solids", Computers and Structures, 54(1):161-166. 
[49] Harmathy, T. Z. (1967). "A comprehensive creep model", Journal of Basic Engineering, Trans ASME,89:496-502.

[50] Haywood, R. W. (1980). Equilibrium Thermodynamics for Engineers and Scientists, J. Wiley and Sons, New York.

[51] Hearn, G. and Testa, R.B. (1991). "Modal analysis for damage detection in struc. tures", Journal of Structural Engineering, 117(10):3042-63.

[52] Hult, J. (1987). "Introduction and General Overview", Continuum Damage Mechanics Theory and Applications, eds: D. Krajcinovic and J. Lemaitre, SpringerVerlag.

[53] Hwang, H., Wang, P. C. and Reich, M. (1983). "Probabilistic models for operational. and accidental loads on seismic category I structures", Report NUREG/CR-3342, US Nuclear Regulatory Commission, Washington, DC. June 1983.

[54] Ihara, C. and Misawa, T. (1991). "Stochastic models related to fatigue damage of" materials", Journal of Energy Resources Tech., 113(4):215-21.

[55] IMSL (1991). IMSL Math/Stat Library, version 2.0, (C)1991, IMSL Inc, Houston, TX.

[56] Ishikawa, H., Tsurui, A., Tanaka H. and Ishikawa, H. (1993). "Reliability assessment of structures based upon probabilistic fracture mechanics", Probab. Engrg. Mech., 8(1):43-56.

[57] Joshi, N. R. (1994). "Statistical analysis of UT corrosion data from plates of a crude oil aboveground storage tank", Materials Evaluation, 52:846-9.

[58] Jun, Z. and Xing, Z. (1995). "The asymptotic study of fatigue crack growth based on damage mechanics", Engineering Fracture Mechanics , 50(1):131-141.

[59] Kachanov, L. M. (1958). "On creep rupture time" Izv. Akad. Nauk. USSR, Otd. Tekhn. Nauk, no 8, pp 26-31

[60] Kachanov, L.M.(1986). Introduction to Continuum Damage Mechanics, Martinus Nijhoff.

[61] Kanninen, M.F. and Popelar, C.H. (1985). Advanced Fracture Mechanics, Oxford University Press.

[62] Kaynak, C. and Ankara, A. (1992). "Short fatigue crack growth in Al 2024-T3 and Al 7075-T6", Engineering Fracture Mechanics, 43(5)769-778.

[63] Kaynak, C., Ankara, A. and Baker, T. J. (1996). "Effects of short cracks on fatigue life calculations". International Journal of Fatigue,18(1):25-31.

[64] Keisler, J., Chopra, O. K. and Shack, W. J. (1994). Statistical Analysis of Fatigue Strain-Life Data for Carbon and Low-Alloy Steels, report NUREG/CR-6237, ANL94/21, Argonne National Lab., Argonne, IL.

[65] Keisler, J., Chopra, O. K. and Shack, W. J. (1995). Fatigue Strain-Life Behavior of Carbon and Low-Alloy Steels, Austenitic Stainless Steels, and Alloy 600 in LWR Environments report NUREG/CR-6335, ANL-95/15, Argonne National Lab., Argonne, IL. 
[66] Klesnil, M., Polak, J. and Liskutin, P. (1984). "Short crack growth close to the fatigue limit in low carbon steel". Scripta Metallurgica, 18(11):1231-1234.

[67] Kloeden, P. E. and Platen, E. (1992). Numerical Solution of Stochastic Differential Equations, Springer-Verlag.

[68] Komp, M.E. (1987). "Atmospheric corrosion ratings of weathering steels - calculation and significance", Materials Performance, 26(7):42-4.

[69] Krajcinovic, D. and Silva, M. A. G. (1982). "Statistical aspects of the continuous damage theory", International Journal of Solids and Structures, 18(7):551-562.

[70] Krajcinovic, D. (1984). "Continuum damage mechanics",Applied Mechanics Reviews,37(1):1-6.

[71] Krajcinovic, D. and Sumarac, D. (1987). "Micromechanics of the damage process". Continuum Damage Mechanics Theory and Applications, eds: D. Krajcinovic and J. Lemaitre, Springer-Verlag.

[72] Krajcinovic, D. and Mastilovic, S. (1995). "Some fundamental issues of damage mechanics". Mechanics of Materials, 21(3):217-230.

[73] Kujawski, D. and Ellyin, F. (1992). "Crack initiation and total fatigue life of a carbon steel in vacuum and air", Journal of Testing and Evaluation, 20(6):391395.

[74] Kutt, T. V. and Bieniek, M. P. (1988). "Cumulative damage and fatigue life prediction", AIAA Journal, 26(2):213-219.

[75] Lassen, T. (1991). "Markov modelling of the fatigue damage in welded structures under in-service inspection", International Journal of Fatigue, 13(5):417-422.

[76] Lawless, J. F. (1982). Statistical Models and Methods for Lifetime Data, John Wiley and Sons, New York, 1982.

[77] Lawrence, M. et al (1990). "Fatigue crack-growth reliability", Journal of Engineering Mechanics, ASCE, 116(3):698-708.

[78] Lehner, J., Lin, C., Pratt, W. and Drouin, M. (1997). "Containment performance perspectives based on IPE resluts". Proc. 24th Water Reactor Safety Information Meeting, NUREG/CP-0157, vol 3, US Nuclear Regulatory Commission, Washinton, DC, pp. 25-34.

[79] Lemaitre, J. (1984). "How to use damage mechanics", Nuclear Engineering and Design, 80:233-245.

[80] Lemaitre, J. (1985). "A continuous damage mechanics model for ductile fracture" ,Journal of Engineering Materials and Technology,107(1):83-89.

[81] Lemaitre, J. (1992). A Course on Damage Mechanics, Springer-Verlag.

[82] LeRoy, G. et al (1981). "A model of ductile fracture based on the nucleation and growth of voids", Acta Metallurgica, 29:1509-1522.

[83] Lin, Y. K. (1967). Probabilistic Theory of Structural Dynamics, McGraw-Hill. 
[84] Lin, Y. K. and Yang, J. N. (1983). "On statistical moments of fatigue crack propagation", Engineering Fracture Mechanics, 18(2):243-256.

[85] Mabuchi, K., Horii, Y., Takahashi, H. and Nagayama, M. (1991). "Effect of temperature and dissolved oxygen on the corrosion behavior of carbon steel in high temperature water", Corrosion, 47(7):500-8.

[86] Majumdar, S., Chopra, O. K. and Shack, W. J. (1993). "Interim failure design curves for carbon, low-alloy, and austenitic stainless steels in LWR environments", Proc. 20th WRSM, vol 3, NUREG/CP-0126, vol 3, March 1993.

[87] Manson, S. S., Freche, J. C. and Ensign, C. R. (1967). "Application of a double linear damage rule to cumulative fatigue", Fatigue Crack Propagation, ASTM STP 415 , pp. 384-412.

[88] Martin, W.S. and Wirsching, P. H. (1991). "Fatigue crack initiation -- propagation reliability model", Journal of Materials in Civil Engineering, ASCE, 3(1):1-18.

[89] Maugin, G. A. (1992). The Thermomechanics of Plasticity and Fracture, Cambridge Univ. Press.

[90] McLellan, A.G. (1980). The Classical Thermodynamics of Deformable Materials, Cambridge Univ. Press.

[91] Miller, K. J. and Zachariah, K. P. (1977). "Cumulative damage laws for fatigue crack initiation and stage I propagation", Journal of Strain Analysis, 12(4):262270.

[92] Min, L., Qing, S. and Qing-xiong, Y. (1996). "Large sample size experimental investigation on the statistical nature of fatigue crack initiation and growth". International Journal of Fatigue, 18(2):87-94.

[93] Mola, E.E., Mellein, B., Rodriguez de Schiapparelli, E.M., Vicente, J.L., Salvarezza, R.C. and Arvia, A.J. (1990). "Stochastic approach for pitting corrosion modeling", Journal of the Electrochemical Society, 137(5):1384-90.

[94] Mori, Y. (1992). Reliability-based Condition Assessment and Life Prediction of Concrete Structures, $\mathrm{PhD}$ thesis, Johns Hopkins University, 1992.

[95] Murtaza, G. and Akid, R. (1995). "Modelling short fatigue crack growth in a heattreated low-alloy steel", International Journal of Fatigue, 17(3):207-214.

[96] Najar, J. (1987). "Continuous damage of brittle solids", Continuous Damage Mechanics, Theory and Applications, ed: D. Krajcinovic and J. Lemaitre, SpringerVerlag.

[97] Naus, D. J., Oland, C. B., Ellingwood, B. R. and Norris, W. E. (1996). "Aging of the containment pressure boundary in light water reactor plants", Proc. Water Reactor Safety Information Meeting, Rockville, MD, October, 1996.

[98] Oland, C. B. and Naus, D. J. (1996). Degradation Assessment Methodology for Application to Steel Containments and Liners of Reinforced Concrete Structures in Nuclear Power Plants, Report, ORNL/ NRC/LTR-95/29, US Nuclear Regulatory Commission, Washington, DC. 
[99] Ortiz, K. and Kiremidijian, A. S. (1986). "Time series analysis of fatigue crack growth rate data", Engineering Fracture Mechanics, 24(5):657-675.

[100] Oswald, G.F. and Schueller, G.I. (1984). "Reliability of deteriorating structures", Engrg. fracture mechanics, 20(3):479-488.

[101] Paas, M. H. J. W., Schreurs, P. J. G. and Brekelmans, W. A. M. (1993). "A continuum approach to brittle and fatigue damage: theory and numerical procedures". International Journal of Solids and Structures, 30(4):579-599.

[102] Pasic, H. (1992). "A unified approach of fracture and damage mechanics to fatigue damage problems". International Journal of Solids and Structures, 29(14/15):19571968.

[103] Prigogine, I. (1967). Introduction to Thermodynamics of Irreversible Processes, 3rd ed., Interscience, New York.

[104] Provan, J. W. (1987). "Probabilistic approaches to the material-related reliability of fracture-sensitive structures", Probabilistic Fracture Mechanics and Reliability, ed: James W. Provan, Martinus Nijhoff Publishers.

[105] Provan, J. W. and Rodriguez, E.S.III (1989). "Part I: Development of a Markovian description of pitting corrosion". Corrosion, 45(3): 178-92.

[106] Pyun, S., Lim, C. and Oriani, R.A. (1992). "The role of hydrogen in the pitting of passivating films on pure iron", Corrosion Science, 33(3):437-44.

[107] Reinhard, G., Radtke, M. and Rammelt, U. (1992). "On the role of salts of weak acids in the chemical passivation of iron and steel in aqueous solutions", Corrosion Science, 33(2):307-13.

[108] Robinson, E. L. (1952). "Effect of temperature variation on the long- time rupture strength of steels", Trans. ASME, 74:777-780.

[109] Rocha, M. M., Schueller, G. I. and Okamura, H. (1993). "The fitting of oneand two-dimensional fatigue crack growth laws", Engineering Fracture Mechanics, 44(3):473-480.

[110] Rogers, W.F. (1990). "Statistical prediction of corrosion failures", Mat'ls Performance, 29(6):40-44.

[111] Sato, N. (1987). "Some concepts of corrosion fundamentals", Corrosion Sc., $27(5): 421-33$.

[112] Schutz, W (1993). "Fatigue life prediction - a review of the state of the art", Structural Failure, Product Liability and Technical Insurance, IV, ed. Rossmanith, Elsevier.

[113] Schweitzer, P.A. (1987). What Every Engineer Should Know About Corrosion, Marcel Dekker.

[114] Schweitzer, P.A. (1988). Corrosion and Corrosion Protection Handbook, Marcel Dekker.

[115] Sears, F. W. and Salinger, G. L. (1975). Thermodynamics, Kinetic Theory and Statistical Thermodynamics. Addison-Wesley. 
[116] Shah, V. N., Smith, S. K. and Sinha, U. P. (1994). "Insights for aging management of light water reactor components", Report NUREG/CR-5314, US Nuclear Regulatory Commission, Washington, DC. March 1994.

[117] Sieniutycz, S. and Salamon, P. (1990). Nonequilibrium Theory and Extremum Principles, Taylor and Francis, New York.

[118] Simo, J. C. and Ju, J. W. (1987)."Strain and stress-based continuum damage models - I", International Journal of Solids and Structures, 23(7):821-840.

[119] Snyder, D. L. and Miller, M. I. (1991). Random Point Processes in Time and Space, 2nd ed. Springer-Verlag, New York.

[120] Spencer, B. F. and Tang, J. (1988). "Markov process model for fatigue crack growth", Journal of Engineering Mechanics, ASCE, 114(12): 2134-57.

[121] Stouffer, D. C. and Dame, T. L. (1996). Inelastic Deformation of Metals : Models, Mechanical Properties, and Metallurgy, Wiley, New York.

[122] Strutt, J. E., Nicholls, J. R. and Barbier, B. (1985). "The prediction of corrosion by statistical analysis of corrosion profiles", Corrosion Science, 25(5):305-15.

[123] Tiejun, W. and Zhiwen, L. (1990). "A continuum damage model for weld heat affected zone under low cycle fatigue loading". Engineering Fracture Mechanics, $37(4): 825-829$.

[124] Tie-Jun, W. (1992). "Unified CDM model and local criterion for ductile fracture I and II",Engineering Fracture Mechanics,42(1):177-183,185-193.

[125] Tie-Jun, W. (1994). "Further investigation of a new continuum damage mechanics criterion for ductile fracture: experimental verification and applica,tions", Engineering Fracture Mechanics,48(2):217-230.

[126] Topper, T. H. and Morrow, J. (1970). Simulation of the Fatigue Behavior at the Notch Root in Spectrum Loaded Notched Members, T.\& A.M. Report no 333, Department of Theoretical and Applied Mechanics, University of Illinois, Urbana, IL.

[127] Truesdell, C.(1984).Rational Thermodynamics,Springer-Verlag.

[128] Vesely, W. E. (1987). "Risk evaluations of aging phenomena: the linear aging reliability model and its extensions". Report NEREG/CR-4769 (EGG-2476). US Nuclear Regulatory Commission, Washington, DC.

[129] Virkler, D.A., Hillberry, B.M. and Goel, P.K. (1979). "The statistical nature of fatigue crack propagation", Journal of Engineering Materials and Technology, 101(2):148-153.

[130] Waterhouse, R. B. (1972). Fretting Corrosion, Pergamon.

[131] Wheeler, O. E. (1972). "Spectrum loading and crack growth", Journal of Basic Engineering, 94:181-186.

[132] Williams, D.E., Westcott, C. and Fleischmann, M. (1985). "Stochastic models of. pitting corrosion of stainless steels, I and II". Journal of the Electrochemical Society $132: 1796-1811$. 
[133] Woo, C.W. and Li, D.L. (1992). "A general stochastic dynamic model of continuum damage mechanics", International Journal of Solids and Structures, 29(23):29212932 .

[134] Woo, C.W. and Li, D.L. (1993a). "A universal physically consistent definition of material damage", International Journal of Solids and Structures, 30(15):20972108.

[135] Woo, C.W. and Li, D.L. (1993b). "Statistical analysis of material damage with changing internal structure." Engineering Fracture Mechanics, 45(2):245-254.

[136] Woods, L. C. (1981). "On the local form of the second law of thermodynamics in continuum mechanics", Quarterly of Applied Mathematics, April 1981, pp. 119-126.

[137] Zhao, H. (1993). "An improved probabilistic model of fatigue crack growth", Engineering Fracture Mechanics, 46(5):773-80. 


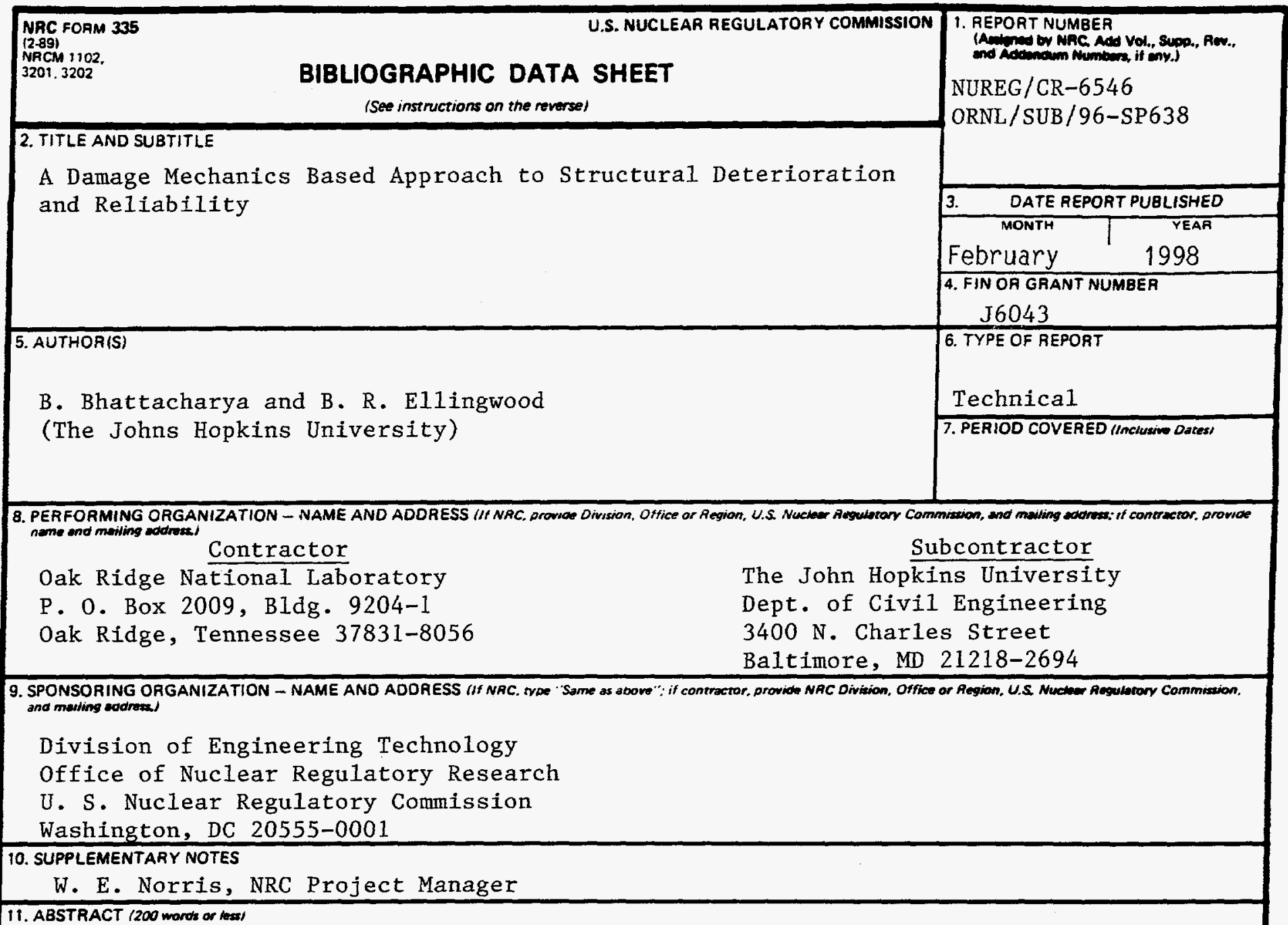

Structural deterioration often occurs without perceptible manifestation. Continuum damage mechanics defines structural damage in terms of the material microstructure, and relates the damage variable to the macroscopic strength or stiffness of the structure. This enables one to predict the state of damage prior to the initiation of a macroscopic flaw, and allows one to estimate residual strength/service life of an existing structure.

The accumulation of damage is a dissipative process that is governed by the laws of thermodynamics. Partial differential equations for damage growth in terms of the Helmholtz free energy are derived from fundamental thermodynamical conditions. Closed-form solutions to the equations are obtained under uniaxial loading for ductile deformation damage as a function of plastic strain, for creep damage as a function of time, and for fatigue damage as function of number of cycles. The proposed damage growth model is extended into the stochastic domain by considering fluctuations in the free energy, and closed-form solutions of the resulting stochastic differential equation are obtained in each of the three cases mentioned above. A reliability analysis of a ring-stiffened cylindrical steel shell subjected to corrosion, accidental pressure, and temperature is performed.

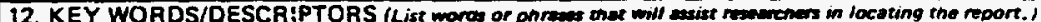

Aging

Containment (stee1)

Continuum Damage Mechanics

Corrosion

Creep

Deformation

Fatigue

Plasticity

Probability Theory
Reliability

Statistics

Structural Engineering

Thermodynamics
13. AVAILABILITY STATEMENT

Unlimited 14. SECUAITY CLASSIFICATION Trhis Penel

Unclassified This Aepert

Unclassified 15. NUMBEA OF PAGES

16. PAICE 